\title{
PREDICTION OF GAS INJECTION PERFORMANCE FOR HETEROGENEOUS RESERVOIRS
}

\author{
FINAL REPORT \\ Petroleum Engineering Department \\ Stanford University \\ Stanford, California 94305-2220
}

Prepared for the U.S. Department of Energy

Under Grant No. DE-FG22-96BC14851

Martin J. Blunt, Franklin M. Orr, Jr., Principal Investigators June 2000 


\section{Disclaimer}

This report was prepared as an account of work sponsored by an agency of the United States Government. Neither the United States Government nor any agency thereof, nor any of their employees, makes any warranty, express or implied, or assumes any legal liability or responsibility for the accuracy, completeness, or usefulness of any information, apparatus, product, or process disclosed, or represents that its use would not infringe privately owned rights. Reference herein to any specific commercial product, process, or service by trade name, trademark, manufacturer, or otherwise does not necessarily constitute or imply its endorsement, recommendation, or favoring by the United States Government or any agency thereof. The views and opinions of authors expressed herein do not necessarily state or reflect those of the United States Government or any agency thereof. 


\section{Acknowledgements}

This report is the result of much effort on the part of a talented group of students, post-docs and research associates. Much of the work reported here was done under the supervision of Martin Blunt, now Professor of Petroleum Engineering at Imperial College. His insight, enthusiasm, and energy contributed greatly to many of the results reported here.

The Stanford effort on streamline simulation methods has been a wonderfully productive collaboration. PhD students Rod Batycky and Marco Thiele, now with Streamsim Technologies, worked with Martin Blunt to develop practical 3D streamline simulation and extend its application to compositional problems. Post-doctoral fellow Yann Gautier, now with the Institut Francais du Petrole, collaborated with Mike Christie, now at Heriot-Watt University, and Martin Blunt to develop a method for nested gridding that can be applied to streamline methods. Together, that group has shown that streamline methods offer huge advantages for many high-resolution, field-scale simulations.

The continued effort to develop analytical methods for solution of one-dimensional compositional flow problems has also been an effective collaboration. The work reported builds on a firm base established by previous DOE-supported projects that worked out the theory of fourcomponent systems. PhD student Yun Wang, now with ARCO, developed the rigorous and fast tie-line intersection approach for calculating minimum miscibility pressures, and Kristian Jessen, a visiting PhD student from the Danish Technical University, worked with PhD student Jichun Zhu and MS student Pavel Ermakov to develop a fully automatic algorithm for calculation of full solutions for one-dimensional displacement of a multicomponent oil by a multicomponent gas. Their work indicates that it should be possible to develop 3D compositional streamline methods based on the fast and accurate analytical solution methods. We are also grateful to Dr. Michael Michelsen of the Danish Technical University and Kristian Jessen's PhD advisor, for allowing us to make use of his very fast and robust algorithms for phase equilibrium calculations.

A key part of better predictions of multiphase flow at field scale is to improve understanding of pore-scale events that influence flow. On the theory side, PhD student Darryl Fenwick, now with the Institut Francais du Petrole, worked with Martin Blunt to build a network model of pore-level events that control three-phase permeability. Three-phase flow experiments to test the theory were conducted by PhD student Akshay Sahni, research associate Dengen Zhou, both now with Chevron, Post-doctoral fellow Jon Burger, now with PTS Laboratories, and Acting Assistant Professor David DiCarlo. Those systematic experiments demonstrate the strong influence of wetting behavior and saturation history on flow behavior. David DiCarlo also deserves special thanks for assembling the report from many different sources.

This project would have been much less productive without the efforts of Yolanda Williams, who somehow keeps our busy research group organized from an administrative and financial standpoint. All of us involved appreciate her dedication.

Finally, all of us gratefully acknowledge the support of the U.S. Department of Energy under Grant No. DE-FG22-96BC14851. We thank Jerry Casteel, Purna Halder and their colleagues for the continued support and encouragement. We are also especially grateful for financial support from the member companies of the SUPRI-C Gas Injection Affiliates Program: ARCO, BP Amoco, 
Chevron, Elf, Exxon, Intevep, Japan National Oil Corporation, Mobil, Norsk Hydro, Saga and Statoil. Without the generous combined support of DOE and industry, this work would not have been possible.

Lynn Orr

Stanford

June 2000 


\section{Abstract}

This final report describes research carried out in the Department of Petroleum Engineering at Stanford University from September 1996 - May 2000 under a three-year grant from the Department of Energy on the "Prediction of Gas Injection Performance for Heterogeneous Reservoirs." The advances from the research include: new tools for streamline-based simulation including the effects of gravity, changing well conditions, and compositional displacements; analytical solutions to $1 \mathrm{D}$ compositional displacements which can speed-up gas injection simulation still further; and modeling and experiments that delineate the physics that is unique to three-phase flow. 


\section{Contents}

$\begin{array}{lll}\text { Disclaimer } & \text { ii }\end{array}$

$\begin{array}{ll}\text { Acknowledgements } & \text { iii }\end{array}$

$\begin{array}{ll}\text { Abstract } & \mathbf{v}\end{array}$

List of Tables $\quad \mathbf{x}$

List of Figures

$\begin{array}{ll}\text { Executive Summary } & 1\end{array}$

1 Introduction $\quad 2$

2 Development of a Streamline Based Reservoir Simulator 5

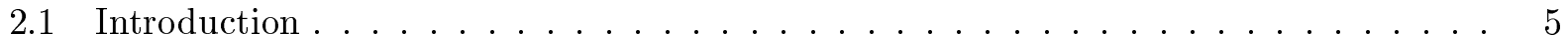

2.2 The Streamline Method . . . . . . . . . . . . . . . . . . 6

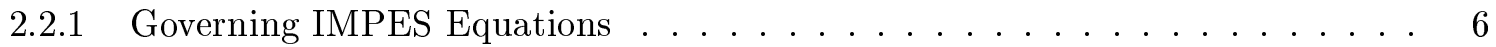

2.2 .2 Coordinate Transform . . . . . . . . . . . . . . . . 7

2.3 Tracer Displacements . . . . . . . . . . . . . . . . . . . . . . . . 9

2.4 First-Contact Miscible Displacements . . . . . . . . . . . . . . . . . . . . . 10

2.5 Two-Phase Displacements . . . . . . . . . . . . . . . . . . . . . . 12

2.6 Million Gridblock Waterflood . . . . . . . . . . . . . . . . . . . . . . 13

2.7 Convergence . . . . . . . . . . . . . . . . . . . . . . . . 14

2.8 Field Example - House Mountain Waterflood . . . . . . . . . . . . . . . 16

2.9 Discussion and Limitations _ . . . . . . . . . . . . . . . . . . . 18

2.10 Conclusions . . . . . . . . . . . . . . . . . . . . 18

2.11 Nomenclature . . . . . . . . . . . . . . . . . . . . . . . . . . . . 19

2.12 Appendix: Tracing Streamlines _. . . . . . . . . . . . . . . . . . 20

3 A Streamline-Based 3D Field-Scale Compositional Reservoir Simulator 22

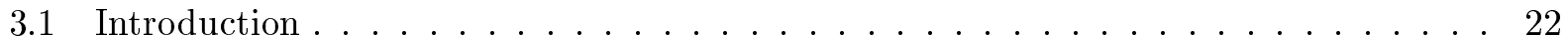

3.2 Compositional Displacements . . . . . . . . . . . . . . . . . . 24

3.3 The Streamline Formulation . . . . . . . . . . . . . . . . . . 25

3.4 One-Dimensional Compositional Solver . . . . . . . . . . . . . . . 26

3.5 Two-Dimensional Solutions . . . . . . . . . . . . . . . . . 27

3.5.1 Three Component Condensing Gas Drive . . . . . . . . . . . . . . 28

3.5.2 Four Component Condensing-Vaporizing Gas Drive . . . . . . . . . . . . . . 29

3.5.3 Sensitivity on Injected Composition. . . . . . . . . . . . . . . . . . . 30

3.6 Three Dimensional Solution . . . . . . . . . . . . . . . . . . . . . 31 
3.7 Timing and Scaling . . . . . . . . . . . . . . . . . . 31

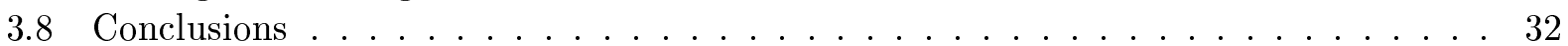

3.9 Nomenclature . . . . . . . . . . . . . . . . . . . . 33

4 Nested Gridding and Streamline Simulation $\quad 42$

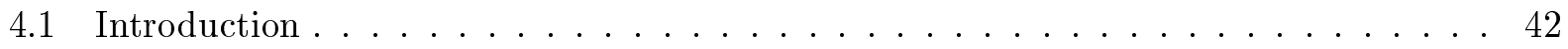

4.2 The Streamline Method . . . . . . . . . . . . . . . . . . . . . . 44

4.3 Description of the New Method . . . . . . . . . . . . . . . . . 46

4.3 .1 The Upscaling Step . . . . . . . . . . . . . . . . . . . . . . . . . . . . . . . . 47

4.3.2 Pressure Solve on the Coarse Grid . . . . . . . . . . . . . . . . . . . . 49

4.3.3 Computation of the Pressure on the Fine Grid. . . . . . . . . . . . . . . . . 49

4.4 CPU Cost Evaluation for the Nested Gridding Method . . . . . . . . . . . . . . . 50

4.5 CPU Requirements of Different Pressure Solvers . . . . . . . . . . . . . . . . . 52

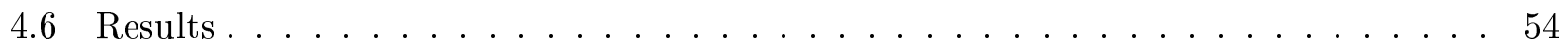

4.6.1 Numerical Results for Two-Dimensional Reservoirs . . . . . . . . . . . . . . . . 55

4.6.2 Numerical Results for Three-Dimensional Reservoirs . . . . . . . . . . . . . . . 59

4.7 Discussion and Conclusions . . . . . . . . . . . . . . . . . . . . 64

5 Analytical Calculation of Minimum Miscibility Pressure for Injection Gases with $\begin{array}{ll}\text { One or Two Components } & 65\end{array}$

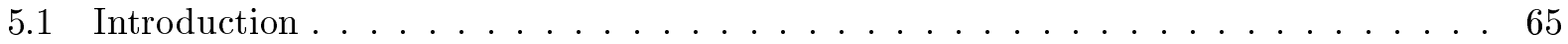

5.2 Mathematical Model . . . . . . . . . . . . . . . . . . . 67

5.3 Modified Negative Flash . . . . . . . . . . . . . . . . . . . . . . . 71

5.4 Analytical Calculation of MMP . . . . . . . . . . . . . . . 75

5.4 Pure $\mathrm{CO}_{2}$ Injection . . . . . . . . . . . . . . . . . . . 75

5.4 .2 Two-Component Gas Injection . . . . . . . . . . . . . . . . 79

5.4 .3 Nontie-line Rarefactions . . . . . . . . . . . . . . . . . . . . . . . . . 82

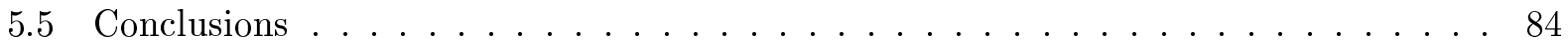

6 Calculation of Minimum Miscibility Pressure for Multicomponent Injection Gases 86

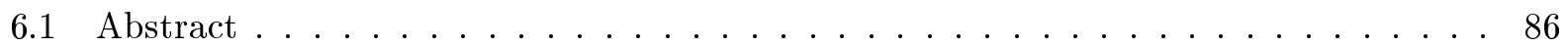

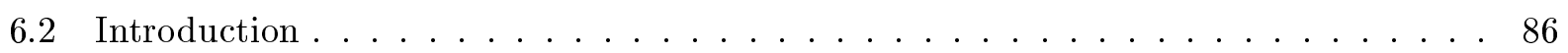

6.3 Mathematical Approach . . . . . . . . . . . . . . . . . . 88

6.4 Examples of MMP Calculations . . . . . . . . . . . . . . . . . . . 90

6.4.1 Four-component System: $\mathrm{CH}_{4} / \mathrm{CO}_{2} / \mathrm{C}_{4} / \mathrm{C}_{10} \ldots \ldots \ldots \ldots$

6.4 .2 Crude Oil Systems . . . . . . . . . . . . . . . . . . . 92

6.5 Discussion . . . . . . . . . . . . . . . . . . . . 93

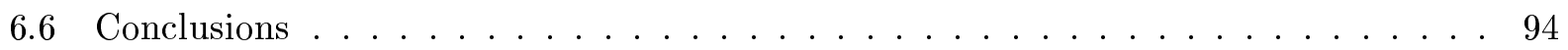

6.7 Nomenclature . . . . . . . . . . . . . . . . . . . . . . 94

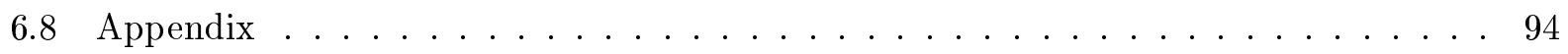

6.9 SI Metric Conversion Factors . . . . . . . . . . . . . . . . . . 95

7 Fast Approximate Solutions for 1D Multicomponent Gas Injection Problems 105

7.1 Introduction . . . . . . . . . . . . . . . . . . 105

7.2 Analytical Theory of 1D Miscible Displacements . . . . . . . . . . . . . 106

7.3 Self-Sharpening Systems . . . . . . . . . . . . . . . . . . . 107

7.4 Solution Example for Fully Self-Sharpening System . . . . . . . . . . . . . . 110

7.5 Systems with Nontie-Line Rarefactions . . . . . . . . . . . . . . . . . . . . . 112

7.6 Prediction of Spreading Waves in 1D Solutions . . . . . . . . . . . . . 113

7.7 The Fanning Rule (Envelope Rule) . . . . . . . . . . . . . . . . . . . . . 114 
7.8 Algorithm for Systems with Nontie-line Rarefactions . . . . . . . . . . . . . . 116

7.9 Solution Example with Nontie-line Rarefaction . . . . . . . . . . . . . 117

7.10 Conclusions . . . . . . . . . . . . . . . . . . . . 117

7.11 Nomenclature . . . . . . . . . . . . . . . . . . . . . . . . 119

8 Use of Network Modeling to Predict Saturation Paths, Relative Permeabilities and Oil Recovery for Three Phase Flow in Porous Media 120

8.1 Introduction . . . . . . . . . . . . . . . . . . . . . . . 120

8.2 Oil Films and Layers . . . . . . . . . . . . . . . . . . . . . . . . . . . . . . . . . . . . . . . . . . . . . . . . . . . . . . . . .

8.3 Conductance of Oil Layers . . . . . . . . . . . . . . . . . . . . . . . . . 124

8.4 Relative Permeability of a Single Pore . . . . . . . . . . . . . . . . . 124

8.5 Network Model . . . . . . . . . . . . . . . . . . . . . . 125

8.6 Paths in Capillary Pressure Space . . . . . . . . . . . . . . . . . . 127

8.7 Self-Consistent Saturation Paths . . . . . . . . . . . . . . . . . . . 128

8.8 1D Numerical Solution . . . . . . . . . . . . . . . . . . . . . . . . . . 129

8.9 Gas Injection . . . . . . . . . . . . . . . . . . . . . . . . . . . . . . . . . . . . . .

8.10 Three Phase Waterflood . . . . . . . . . . . . . . . . . 137

8.11 Conclusions . . . . . . . . . . . . . . . . . . . . . 138

9 Three-Phase Flow Experiments $\quad 142$

9.1 Motivation . . . . . . . . . . . . . . . . . . . . . 142

9.2 Literature Review . . . . . . . . . . . . . . . . . . . . . . . . . . 142

9.3 Dynamic Displacement Experiments . . . . . . . . . . . . . . . . . . 143

9.4 Nomenclature . . . . . . . . . . . . . . . . . . . . . 153

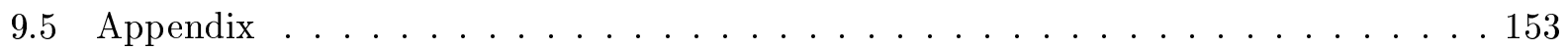

10 Measurement of Three Phase Relative Permeability during Gravity Drainage using CT Scanning $\quad \mathbf{1 5 5}$

10.1 Introduction . . . . . . . . . . . . . . . . . . . . . 155

10.1.1 Gravity Drainage Experiments . . . . . . . . . . . . . . 156

10.1.2 Calculating Three Phase Relative Permeability . . . . . . . . . . . . . 156

10.2 Our Approach to the Three Phase Flow Problem . . . . . . . . . . . . . . . 157

10.2.1 Three Phase Gravity Drainage . . . . . . . . . . . . . . . . . 157

10.2.2 Analysis of Gravity Drainage Experiments . . . . . . . . . . . . . . . 157

10.3 Dual Energy CT scanning and Experimental Design _ . . . . . . . . . . . . 160

10.3.1 Experimental Procedure . . . . . . . . . . . . . . . . 161

10.3.2 Analog Experiments . . . . . . . . . . . . . . . . . 162

10.3.3 Measuring the Spreading Coefficient . . . . . . . . . . . . . . . 163

10.3.4 In-situ Saturation Measurements from Scanned Images . . . . . . . . . . . . 164

10.4 Three Phase Gravity Drainage Experiments . . . . . . . . . . . . . . . . . 167

10.5 Results and Discussion . . . . . . . . . . . . . . . . . . 167

10.6 Summary and Conclusions . . . . . . . . . . . . . . . . . . 169

10.7 Nomenclature . . . . . . . . . . . . . . . . . . . . . . . 169

10.8 Appendix . . . . . . . . . . . . . . . . . . . . . . 172

10.8.1 Three Phase Saturation Calculation _ . . . . . . . . . . . . . 172

10.8.2 Calculating Porosity . . . . . . . . . . . . . . . 173

10.8 .3 Error Analysis . . . . . . . . . . . . . . . . . . 173 
11 Three-Phase Relative Permeability of Water-Wet, Oil-Wet, and Mixed-Wet Sandpacks $\quad \mathbf{1 7 4}$

11.1 Introduction $\ldots \ldots \ldots \ldots \ldots \ldots \ldots \ldots$

11.2 Materials and Methods . . . . . . . . . . . . . . . . . . . 175

11.2.1 Capillary Pressure Curves . . . . . . . . . . . . . . . . . 176

11.2.2 Oil and Water Relative Permeabilities . . . . . . . . . . . . . . . 177

11.2.3 Gas Relative Permeability . . . . . . . . . . . . . . . . . . . . . 179

11.3 Results . . . . . . . . . . . . . . . . . . . . . . . 180

11.3.1 Capillary Pressure Curves . . . . . . . . . . . . . . . . . . . . . . . . . . . . . . . . . . . . . .

11.3.2 Three-Phase Relative Permeability . . . . . . . . . . . . . . . . . 181

11.4 Pore Scale Explanation . . . . . . . . . . . . . . . . . . . . . 185

11.5 Discussion . . . . . . . . . . . . . . . . . . . . . . . . 191

11.6 Conclusions . . . . . . . . . . . . . . . . . . . . . . . . 191

11.7 Nomenclature . . . . . . . . . . . . . . . . . . . . . . . 192 


\section{List of Tables}

4.1 CPU time (in $s$ ) and memory (in $\mathrm{MB}$ ) comparisons for a pressure solve on $3 D$ permeability fields of varying size. . . . . . . . . . . . . . . . 54

4.2 Speed-up factor and memory requirements of the nested grid method for a pressure solve and for the whole simulation on the two $2 D$ examples presented in the chapter. 59

4.3 Speed-up factor and memory requirements of the nested gridding method for a pressure solve and for the whole simulation on the $3 D$ example presented in the chapter. The numbers in parenthesis in the third column are speed-up factors for the case

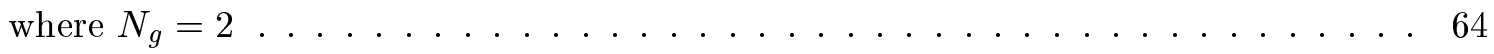

5.1 Oil and gas compositions for pure $\mathrm{CO}_{2}$ injection, $\mathrm{T}=160^{\circ} \mathrm{F} \ldots \ldots \ldots$

5.2 Key tie-line structure for pure $\mathrm{CO}_{2}$ injection. . . . . . . . . . . . . 76

5.3 Oil and gas compositions for a four-component system, $\mathrm{T}=160^{\circ} \mathrm{F} \ldots \ldots \ldots$

5.4 Tie-line structure for two-component injection. . . . . . . . . . . . . . . 85

5.5 Comparison of the initial tie lines obtained from three different approaches for the system shown in Fig. 5.11. . . . . . . . . . . . . . . . . . 85

5.6 Comparison of CPU times between the numerical and analytical approaches. . . . . 85

7.1 Characterized fluid description (SRK - Equation of state) . . . . . . . . . . . 111

7.2 MOC solution for displacement of Oil A by Gas A at $275 \mathrm{~atm}$ and $387 \mathrm{~K}$. . . . . . 111

7.3 Summary of the fanning (envelope) rule. . . . . . . . . . . . 116

7.4 Comparison of tie line found by MOC integration and by the tie line intersection approach. Gas A displaced by pure $\mathrm{N}_{2}$ at 275 atm and 387 K. . . . . . . . . . 118

9.1 Polynomial fit to relative permeability . . . . . . . . . . . . . . . 145

10.1 Three Phase Gravity Drainage Experiments. $S_{o i}$ is the initial oil saturation averaged over the whole core. $S_{\text {of }}$ is the final oil saturation at the end of the experiment averaged over the top half of the column (top $45 \mathrm{~cm}$ ). $S_{w f}$ is the final average water saturation in the top half of the column. The $*$ indicates waterflood residual oil $\left(S_{\text {or }}\right) 158$

10.2 Fluid Properties . . . . . . . . . . . . . . . . . . . . . 161

10.3 Interfacial tensions and spreading coefficient . . . . . . . . . . . . . 164

11.1 Interfacial tensions $\ldots \ldots \ldots \ldots \ldots$

11.2 Wettability Indices . . . . . . . . . . . . . . . . . . . . . 180

11.3 Experiments . . . . . . . . . . . . . . . . . . 181 


\section{List of Figures}

2.1 Distribution of a tracer slug using (a) the streamline method and (b) ECLIPSEIMPES. $m_{c}$ is the center of mass of the tracer slug. (c) Conceptual picture illustrating the final position of a fluid element at the end of time interval $\Delta t$ by transporting along a streamline vs. the underlying Cartesian grid. . . . . . . . . . . . . . 9

2.2 Comparison of tracer profiles in a $250 \times 100$ heterogeneous domain using the streamline simulator: (a) mapping an analytical tracer solution along streamlines, (b) mapping a SPU (numerical) solution with 1 remapping step, (c) mapping a SPU solution with 10 remapping steps, (d) mapping a TVD (numerical) solution with 1 remapping step, (e) mapping a TVD solution with 10 remapping steps. . . . . . . . . . . . . 10

2.3 Comparison of FCM solvent profiles between 3DSL and ECLIPSE at $t_{D}=0.6$ in a $125 \times 50$ heterogeneous model at three gravity numbers. Injection is into the lower 10 left gridblocks and production is from the lower 10 right gridblocks. . . . . . . . . 11

2.4 FCM displacement recovery comparisons between 3DSL and ECLIPSE, for three different gravity numbers. . . . . . . . . . . . . . . . . . . 12

2.5 Comparison of water saturation profiles between 3DSL and ECLIPSE at $t_{D}=0.4$ in a $250 \times 75$ heterogeneous model at three gravity numbers. Injection is into the upper 10 left gridblocks and production is from the upper 10 right gridblocks. . . . . . . . 13

2.6 Water saturations predicted using 3DSL with and without gravity (100,000 gridblocks). Injection is in the central bottom two gridblocks, production is from the top two gridblocks of each corner. . . . . . . . . . . . . . . . . . . . . 14

2.7 Waterflood displacement recovery comparison between 3DSL and ECLIPSE for a 3D model (100,000 gridblocks) with two different gravity numbers. . . . . . . . . . . 15

2.8 Waterflood oil recovery comparisons between 3DSL million gridblock model and upscaled ECLIPSE-IMPES and 3DSL models. . . . . . . . . . . . . . . . 15

2.9 Convergence of oil recovery curve as maximum front speed is reduced from 250 to 15 gridblocks per time step, for $250 \times 75$ waterflood displacement, $N_{g}=0.4$. . . . . 16

2.10 Comparison of historical production rate data with history matches for the streamline simulator (3DSL) and a conventional implicit simulator (MORES) . . . . . . . . . 17

2.11 Schematic of a streamline path through a 2D gridblock of dimensions $\Delta x$ by $\Delta y$. . 20

3.1 One-dimensional, TVD solutions at $t_{D}=0.5$ with 100 grid blocks using UTCOMP (v.3.2 w/ automatic time stepping) and the $1 \mathrm{D}$ compositional simulator $(\mathrm{CFL}=0.5)$ used in this work for pure $\mathrm{CO}_{2}$ injection into a $30 / 70$ mixture of $\mathrm{CH}_{4}$ and $\mathrm{C}_{10}$ at 1600 psia and $160^{\circ}$ F. . . . . . . . . . . . . . . . 27

3.2 One-dimensional mobility ratio profile for the three component condensing problem

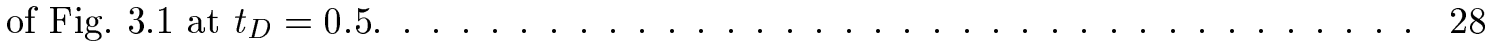

3.3 Permeability field $(62 \times 25)$ and spatial distribution of $\mathrm{CO}_{2}$ for $3 \mathrm{DSL}$ and UTCOMP

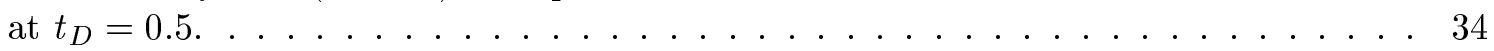

3.4 Cumulative recoveries of $\mathrm{CH}_{4}$ plus $\mathrm{C}_{10}$ for 3DSL and UTCOMP. . . . . . . . . 35 
3.5 Spatial distribution of $\mathrm{CO}_{2}$ for 3DSL at $t_{D}=0.5$ for grids $124 \times 50$ and $248 \times 100$ compare to the UTCOMP solution for grid $124 \times 50 \ldots \ldots \ldots \ldots$

3.6 One-dimensional, TVD solution with 100 gridblocks using UTCOMP (v.3.2 w/ automatic time stepping) and TVD solutions with 100 and 500 gridblocks using 3DSL $(\mathrm{CFL}=0.25)$ for $\mathrm{CH}_{4} / \mathrm{C}_{3}(65 / 35)$ injection into a $20 / 40 / 40$ mixture of $\mathrm{CH}_{4} / \mathrm{C}_{6} / \mathrm{C}_{16}$

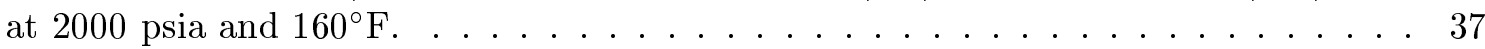

3.7 Saturations maps at $t_{D}=0.4$ for the displacement of a $20 / 40 / 40$ mixture of $\mathrm{CH}_{4} / \mathrm{C}_{6} / \mathrm{C}_{16}$ at 2000 psia and $160^{\circ} \mathrm{F}$ by a $65 / 35$ mixture of $\mathrm{CH}_{4} / \mathrm{C}_{3}$ through a heterogeneous cross-section with $125 \times 50$ gridblocks. . . . . . . . . . . . . 38

3.8 Sensitivity of the displacement of a $20 / 40 / 40$ mixture of $\mathrm{CH}_{4} / \mathrm{C}_{6} / \mathrm{C}_{16}$ at 2000 psia and $160^{\circ} \mathrm{F}$ on the enrichment level of the injected mixture of $\mathrm{CH}_{4} / \mathrm{C}_{3}$. The permeability field has 5 orders of magnitude difference in permeability. The maps show spatial distribution of $\mathrm{C}_{3}$. Recoveries are for $\mathrm{C}_{6}$ plus $\mathrm{C}_{16} \ldots \ldots \ldots$. . . . . . 39

3.93 D solutions with $180 \times 180 \times 16=518,400$ gridblocks and with $60 \times 60 \times 8=28,800$ gridblocks for the $4 \mathrm{C}$ problem of Fig. 3.6. . . . . . . . . . . . . . 40

3.10 Scaling of compositional solution. . . . . . . . . . . . . . . . . . 41

4.1 Description of the streamline method. Consider a permeability map (a) with injector and a producer wells. Solve the pressure field (b). Compute the velocity field and trace streamlines $(c)$. Move saturation along streamlines and compute the values of the saturation on the grid $(d) \ldots \ldots \ldots \ldots \ldots$

4.2 Representation of the coarse grid superimposed on a fine grid. The right figure represents a particular coarse grid block . . . . . . . . . . . . . . . 46

4.3 Upscaling of the transmissivities: the fine transmissivity map is divided into coarse grid blocks. The pressure equation is solved independently on each coarse grid block with a no flux boundary on all the faces except two opposing faces across which a prescribed pressure drop is applied. The upscaled values of the transmissivities are then obtained from the computation of the flux across the system. . . . . . . . . . 48

4.4 Upscaling of the transmissivity in the three directions $(a-c)$ where a constant pressure drop $\Delta P_{d}$ is applied on the direction $d$ and the gravity is set to $g=0$. (d) depicts the upscaling of the gravity transmissivity where $\Delta P_{z}=0$ and $g \neq 0 \ldots \ldots$

4.5 Computation of the fine grid pressure field using the pressure of the coarse grid and the fluxes coming from the neighboring coarse grid blocks. The fluxes are computed using Darcy's law and are weighted by the inter-blocks transmissivities. . . . . . . .

4.6 Description of the different steps of the nested gridding method. We first consider a fine description of the reservoir (a). The transmissivities are then upscaled on a coarse scale (b) and a pressure solution on the coarse grid is computed (c). The next step is the computation of the pressure on the fine grid (d) which leads to the velocity field. The streamlines are traced from the injector to the producer $(e)$ and saturation is moved using the classical streamline method $(f) \ldots \ldots \ldots \ldots$

$4.72 D$ grid with $n_{x}=5, n_{y}=5$ and the corresponding transmissivity sparse matrix. Only nonzero elements are represented. . . . . . . . . . . . . . . . 52

$4.83 D$ grid with $n_{x}=2, n_{y}=5$ and $n_{z}=5$ and the corresponding sparse matrix. Only nonzero elements are represented. . . . . . . . . . . . . . . . 53

4.9 Heterogeneous permeability fields (a), in a log-scale, used to compare the CPU time needed to solve the pressure. There are all extracted from a $500 \times 500$ permeability field. (b) is the CPU time comparison for a pressure solve using different solvers for $2 D$ problems. . . . . . . . . . . . . . . . . . . . . 
4.10 Permeability field containing $270 \times 270$ grid blocks with two facies. Permeability: $k$ (black) $=100 \mathrm{mD}$ and $k$ (white $)=0.1 \mathrm{mD}$. Proportion of black facies: $90 \%$. Correlation length: 10 grid blocks in the $x$ and $y$ directions with an anisotropy angle

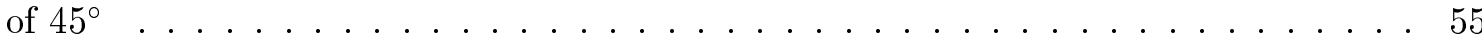

4.11 Fractional flow of oil at the producer for different upscaling factors and for the three methods (fine grid, nested gridding and upscaling). Case RES1. . . . . . . . . .

4.12 Water saturation maps from the fine grid simulation (a), from the nested gridding simulation (b) and from the simulation on the upscaled absolute permeabilities (c) for 0.35 PVI. The upscaling factor is $9 \times 9$. Case RES1 . . . . . . . . . . . . 56

4.13 Permeability field containing $270 \times 270$ grid blocks with two facies. Permeability: $k$ (black) $=100 \mathrm{mD}$ and $k$ (white) $=0.1 \mathrm{mD}$. Proportion of black facies: $90 \%$. Correlation length: 20 grid blocks in the $x$ and 2 grid blocks in the $y$ direction. . . . 57

4.14 Fractional flow of oil at the producer for different upscaling factors and for the three methods (fine grid, nested gridding and upscaling). Case RES2 . . . . . . . . . . 57

4.15 Water saturation maps from the fine grid simulation (a), from the nested gridding simulation (b) and from the simulation on the upscaled absolute permeabilities $(c)$ for 0.8 PVI. The upscaling factor is $27 \times 27$. Case RES2 . . . . . . . . . . . . 58

4.16 Case RES3. $99 \times 99 \times 99$ permeability field. The two wells are located on two opposing corners of the reservoir and they penetrate all the layers. The correlation length is equal to 2 grid blocks in all 3 directions. The permeability of the black facies is $100 \mathrm{mD}$ and $0.1 \mathrm{mD}$ for the white facies. . . . . . . . . . . . . .

4.17 Fractional flow of oil at the producer for different upscaling factors and for the three methods (fine grid, nested gridding and upscaling) for the case RES3. The gravity number is set to $N_{g}=0 \ldots \ldots \ldots \ldots \ldots$

4.18 Fractional flow of oil at the producer for different upscaling factors and for the three methods (fine grid, nested gridding and upscaling) for the case RES3. The gravity number is set to $N_{g}=2 \ldots \ldots \ldots \ldots \ldots \ldots$

4.19 Saturation maps for 0.15 PVI using the three methods (fine grid, nested gridding and upscaling) with an upscaling factor equal to $(3 \times 3 \times 3)$. The gravity number is set to $N_{g}=0 \ldots \ldots \ldots \ldots \ldots \ldots \ldots$

4.20 Saturation maps for 0.15 PVI using the three methods (fine grid, nested gridding and upscaling) with an upscaling factor equal to $(3 \times 3 \times 3)$. The gravity number is set to $N_{g}=2 \ldots \ldots \ldots \ldots \ldots \ldots \ldots$

5.1 Composition paths in a four-component system with constant K-values (Points a, b, c, $\mathbf{d}$ refer to composition points in Fig. 5.2. . . . . . . . . . . . . . 68

5.2 Variation of the nontie-line eigenvalue alonf the nontie-line path. . . . . . . . . 69

5.3 Key tie lines for a displacement of a three-component oil (composition o) by a twocomponent gas (composition g) at $160^{\circ} \mathrm{F}\left(71.1^{\circ} \mathrm{C}\right), 1600$ psia $(11.0 \mathrm{MPa})$. The crossover tie line intersects extensions of the initial oil and injection gas tie lines. See Table 5.3 for oil and gas compositions. . . . . . . . . . . . . . . 70

5.4 Composition for test of the modified negative flash algorithm $\left(\mathrm{T}=160^{\circ} \mathrm{F}\left(71.1^{\circ} \mathrm{C}\right)\right.$, $\mathrm{P}=1600 \mathrm{psia}(11.0 \mathrm{MPa})) \ldots \ldots \ldots \ldots \ldots \ldots \ldots \ldots$

5.5 Convergence of the modified negative flash. . . . . . . . . . . . . . . . 74

5.6 Dependence of key tie line lengths on pressure for displacement of a six-component oil by $\mathrm{CO}_{2}$ at $160^{\circ} \mathrm{F}\left(71.1^{\circ} \mathrm{C}\right)$. The MMP is 2380 psia (16.4 MPa). See Table 5.1 for oil and gas compositions. . . . . . . . . . . . . . . . . . 78

5.7 Results of one-dimensional compositional simulations with 800 grid blocks and a Courant number of 0.2, for the systems of Fig. 5.6, Fig. 5.8 and Fig. 5.9 . . . . . . . 78 
5.8 Key tie line lengths for $\mathrm{CO}_{2}$ displacement of a ten-component oil at $120^{\circ} \mathrm{F}\left(48.9^{\circ}\right.$ C). The MMP is 1466 psia $(10.1 \mathrm{MPa}) . \ldots \ldots \ldots \ldots$. . . . . . . . . . 79

5.9 Key tie line lengths for a two-component gas displacement of a three-component oil at $160^{\circ} \mathrm{F}\left(71.1^{\circ} \mathrm{C}\right)$, (see Fig. 5.3). The MMP is $2297 \mathrm{psia}(15.9 \mathrm{MPa}) \ldots$. . . . . . . 81

5.10 Effect of $\mathrm{CH}_{4}$ contamination of $\mathrm{CO}_{2}$ on MMP for the displacement of a $\mathrm{CH}_{4} / \mathrm{C}_{4} / \mathrm{C}_{10}$ mixture at $160^{\circ} \mathrm{F}\left(71.1^{\circ} \mathrm{C}\right)$. See Table 5.3 for the oil composition and Fig. 5.3 for the phase diagram. . . . . . . . . . . . . . . . . . .

5.11 Displacement of $20 \% \mathrm{CH}_{4}, 40 \% \mathrm{C}_{6}$ and $40 \% \mathrm{C}_{16}$ by $90 \% \mathrm{CH}_{4}$ and $10 \% \mathrm{C}_{3}$ at $\mathrm{P}=$ 2000 psia and $\mathrm{T}=200^{\circ} \mathrm{F}$. There is a rarefaction between crossover tie line $\mathrm{C}$ and initial tie line $\mathrm{O}$.

5.12 Numerical and analytical solutions for a system with a rarefaction along a nontie-line path. . . . . . . . . . . . . . . . . .

5.13 Displacement of $20 \% \mathrm{CH}_{4}, 40 \% \mathrm{C}_{6}$ and $40 \% \mathrm{C}_{16}$ by $90 \% \mathrm{CH}_{4}$ and $10 \% \mathrm{C}_{3}$ at $\mathrm{T}=$ $200^{\circ}$ F. MMP $=4264$ psia $(29.4 \mathrm{MPa}) \ldots \ldots \ldots \ldots$

6.1 Key tie lines intersect each other for a displacement of oil $o$ by gas $g$. . . . . . . 98

6.2 Comparison of MMPs calculated by forward contacts in a single mixing cell and by the tie line intersection method. . . . . . . . . . . . . . . . . . 98

6.3 Composition paths and crossover tie line surface for the displacement of oil $O(40 \%$ $\mathrm{CH}_{4}, 5 \% \mathrm{CO}_{2}, 20 \% \mathrm{C}_{4}$ and $\left.35 \% \mathrm{C}_{10}\right)$ by $I_{1}$ to $I_{4}$ at 1600 psia and $160 \mathrm{~F} \ldots \ldots$. . . . 99

6.4 Compositional simulation results for the displacement of $40 \% \mathrm{CH}_{4}, 5 \% \mathrm{CO}_{2}, 20 \%$ $\mathrm{C}_{4}$ and $35 \% \mathrm{C}_{10}$ by $50 \% \mathrm{CO}_{2}$ and $50 \% \mathrm{CH}_{4}$ at $160 \mathrm{~F}$. The calculated MMP is 2500

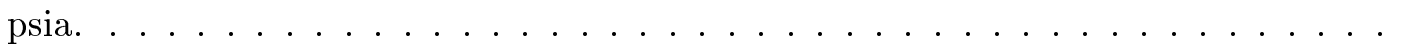

6.5 Convergence of reverse contacts to the injection gas tie line and forward contacts to the initial oil tie line (symbols indicate the phase compositions obtained by a negative flash). . . . . . . . . . . . . . . . . . . 100

6.6 Tie line lengths for rich gas displacement of an eight-component oil (Hearn and Whitson [83]) at 212F. The calculated MMP is 3279 psia. . . . . . . . . . . . 100

6.7 Tie line lengths for lean gas displacement of an eight-component oil (Hearn and Whitson [83]) at 212F. The calculated MMP is 5582 psia. . . . . . . . . . . . . 101

6.8 Tie line lengths for rich gas displacement of a 15-component oil (Hearn and Whitson [83]) at 212F. The calculated MMP is 3115 psia. . . . . . . . . . . . . . . 101

6.9 Tie line lengths for lean gas displacement of a 15-component oil (Hearn and Whitson [83]) at 212F. The calculated MMP is 5253 psia. . . . . . . . . . . . . . 102

6.10 Tie line lengths for displacement of oil A (Zick [230]) by solvent A at 185F. The calculated MMP is 2169 psia. . . . . . . . . . . . . . . . . . . . 102

6.11 Tie line lengths for displacement of oil A (Zick [230]) by $20 \% \mathrm{CH}_{4}$ and $80 \%$ solvent A at $185 \mathrm{~F}$. The calculated MMP is 3013 psia. . . . . . . . . . . . 103

6.12 Tie line lengths for displacement of oil A (Zick [230]) by $23 \% \mathrm{CH}_{4}$ and $77 \%$ solvent A at $185 \mathrm{~F}$. The calculated MMP is 3283 psia. . . . . . . . . . . . . . 103

6.13 Comparison between analytically calculated MMPs with slim tube test results. . . . 104

7.1 Construction of tangent shocks. . . . . . . . . . . . . . . . 108

7.2 Illustration of downstream solution path construction. . . . . . . . . . . . 109

7.3 Comparison of analytical and numerical saturation profiles for displacement of Oil A by Gas A at $275 \mathrm{~atm}$ and $387.45 \mathrm{~K}$. The finite difference (FD) solutions were obtained with 100,1000, and 10,000 grid blocks and $\Delta z / \Delta t=10 \ldots \ldots \ldots$. . . 112

7.4 Illustration of envelope curve. . . . . . . . . . . . . . . . . . . . 114

7.5 Vaporizing wave with liquid side envelope. Upstream (u), Downstream (d). . . . . 115 
7.6 Condensing wave with vapor side envelope. . . . . . . . . . . . . . . . 115

7.7 Condensing wave with liquid side envelope. . . . . . . . . . . . . . . 115

7.8 Vaporizing wave with vapor side envelope. . . . . . . . . . . . . . 116

7.9 Comparison of analytical solutions (with and without integration) with numerical saturation profiles for the displacement of Oil A by pure $\mathrm{n}_{2}$ at 275 atm and $387.45 \mathrm{~K}$. The finite difference (FD) solutions were obtained using 100 and 10,000 grid blocks

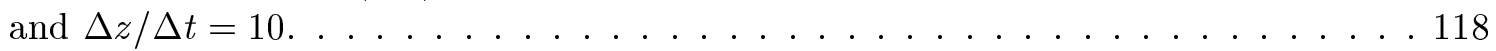

8.1 Three phases in a corner with half angle $\beta . \ldots \ldots \ldots \ldots \ldots \ldots$

8.2 Oil relative permeabilities measured in a water-wet system. (from Grader \& O'Meara [76] using a glass bead pack and analogue fluids. Included is a line of slope 1.9 on a log-log

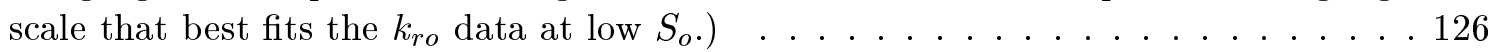

8.3 Different paths in capillary pressure space. The $R=R_{c}$ line separates the regions of oil layer stability. Path BCD represents gas invasion into high $P_{\text {cow }}$ corresponding to high $S_{o}$, followed by imbibition of water. Path AD represents gas invasion into low $P_{\text {cow }}$ corresponding to residual oil saturation after water invasion. . . . . . . . 127

8.4 Schematic diagram of different scales in multiphase flow in porous media. At the field scale $(100-1000 \mathrm{~m})$, the saturation profile is dominated by viscous forces. At much smaller scales $\left(10^{-3}-10^{-2} \mathrm{~m}\right)$, saturations are relatively constant and flow is dominated by capillary forces. Around a length scale of a meter, capillary and viscous forces are approximately the same. . . . . . . . . . . . . . 128

8.5 Schematic diagram of iteration procedure to obtain self-consistent relative permeability values. (1) A saturation path through the three phase region is guessed and followed with the network model. (2) During the displacement, three phase relative permeability values are calculated. (3) These relative permeability values are input into a 1-D numerical simulator with selected initial conditions, injection conditions, and fluid properties. (4) The 1-D simulator generates another saturation path. (5) This path is compared to the proposed path with the network model. (6) If the paths do not coincide, repeat the procedure, using the path from step 4 in the network model. . . . . . . . . . . . . . . . . . . . . . . . . . . . . . . . . .

8.6 Self-consistent saturation paths for gas injection into oil and water. . . . . . . . . 132

8.7 Capillary pressure paths for gas injection into different values of initial oil saturation

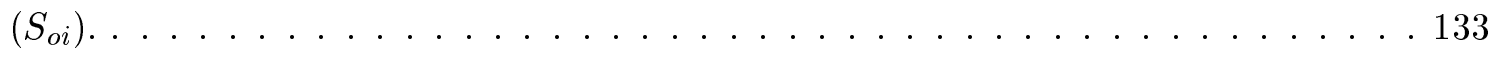

8.8 Self-consistent $k_{r g}$ curves for the saturation paths in Fig. 8.6. . . . . . . . . 133

8.9 Self-consistent $k_{r w}$ curves for the saturation paths in Fig. 8.6. . . . . . . . . . 134

8.10 Self-consistent $k_{r o}$ curves for the saturation paths in Fig. 8.6. . . . . . . . . 135

8.11 Recovery curves for gas injection at $C_{s}^{e q}=0 \ldots \ldots \ldots \ldots \ldots \ldots$

8.12 Self-consistent saturation paths for gas injection into oil and water with $C_{s}^{e q}=-4$

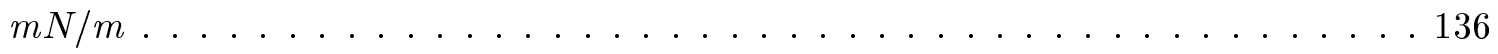

8.13 Self-consistent $k_{r o}$ curves for the saturation paths in Fig. 8.12. . . . . . . . 137

8.14 Recovery curves for gas injection at $C_{s}^{e q}=-4 m N / m . \ldots \ldots \ldots$

8.15 Self-consistent saturation path for waterflooding a gas condensate reservoir. . . . . 139

8.16 Capillary pressure path for the waterflood of a gas condensate reservoir. . . . . . . 139

8.17 Self-consistent $k_{r o}$ curves for the saturation paths in Fig. 8.15 . . . . . . . . . . . 140

8.18 Recovery curves for water injection of gas condensate reservoir. . . . . . . . . . . 140

9.1 Saturation trajectories from Grader and O'Meara's [76] experiments . . . . . . . . 144

9.2 Measured and fitted relative permeabilities . . . . . . . . . . . . . 145 
9.3 Rarefaction curves for the polynomial relative permeabilities fit to Grader and O'Meara's [76] experiments . . . . . . . . . . . . . . . . . . 147

9.4 Predicted and measured saturation paths for Grader and O'Meara's [76] run \#9 . . 149

9.5 Predicted and measured saturation paths for Grader and O'Meara's [76] run \#8 . . 150

9.6 Predicted and measured saturation paths for Grader and O'Meara's [76] run \#5 . . 151

9.7 Predicted and measured recoveries for Grader and O'Meara's [76] run \#5 . . . . . . 152

10.1 Calibration and porosity measurements from the CT Scanner. . . . . . . . . . 160

10.2 Results of sieve analysis for the sand used in this study. . . . . . . . . . . . . . 161

10.3 In-situ saturation measurements at different locations along the length of the core after 2.50 hours of drainage. . . . . . . . . . . . . . . . . . . 164

10.4 Water and air saturation profiles as a function of time and distance along the length of the core. . . . . . . . . . . . . . . . . . . . 165

10.5 Water relative permeability as a function of water saturation. The straight line is a best fit through the data on the doubly logarithmic axes. . . . . . . . . . . 165

10.6 Saturation path and oil saturation profile for experiment $1 . \ldots . \ldots 166$

10.7 Water and air saturation profiles as a function of time and distance for experiment 1. 166

10.8 Water relative permeability as a function of water saturation. The straight line in the top figure is a best fit through the data. Notice that the results of the three experiments lie on a single curve, which is similar to the two phase results in Fig. 10.5.168

10.9 Measured oil relative permeabilities. The straight lines are best fits to the data at low oil saturation. Notice that all the curves show an approximately quadratic form for the oil relative permeability at low oil saturations, except for the non-spreading decane system. . . . . . . . . . . . . . . . . . . 170

10.10Oil relative permeability from Naylor et al.. [147] . . . . . . . . . . . . . . . 171

10.11Oil relative permeability from Grader and O'Meara. [76] . . . . . . . . . . . . 171

10.12 Fluid distribution in the corner of a pore space. . . . . . . . . . . . . . . 171

11.1 Oil (octane) and water saturation profiles versus time during gravity drainage. The sand was water-wet and the initial condition was connate water saturation $S_{w c}$. Oil and water permeabilities can be calculated directly from the measured distributions. 178

11.2 Measured two-phase (water/oil) capillary pressure curves and three-phase oil and water relative permeabilities with n-octane as the oil for water-wet, oil-wet, mixedwet, and fractionally-wet sand. Open symbols are drainages which started from residual oil saturation $\left(S_{o r}\right)$, and closed symbols are drainages which started from connate water saturation $\left(S_{w c}\right) \ldots \ldots \ldots \ldots$. . . . . . . . . . . . . . . . . . . .

11.3 Measured oil relative permeabilities with hexane, octane, and decane as the oil in water-wet sand and with octane in water-wet sandstone[186] during three-phase gravity drainage. . . . . . . . . . . . . . . . . . . . . . 183

11.4 Measured water relative permeabilities for a) the two-phase (gas/water) system and for $b$ ) the three-phase (gas/oil/water) system from gravity drainages in water-wet sand. . . . . . . . . . . . . . . . . . . . . . . 184

11.5 Measured gas relative permeabilities for a) the two-phase gas/water system for media of varying wettability and the two-phase gas/oil system for oil-wet media, and for b) the three-phase gas/oil/water system for water-wet and oil-wet media. The gas relative permeability in oil-wet sand is roughly a factor of two smaller than that for identical water-wet sand for two-phase gas/water systems. . . . . . . . . . . . . . . 184

11.6 Configuration of fluids on : a) a flat water-wet surface, b) a flat oil-wet surface (shown bold). . . . . . . . . . . . . . . . . . . 187 
11.7 Possible configuration of fluids in a pore space corner of half-angle $\beta$ : a) water-wet surfaces, b) oil-wet surfaces (shown bold). Note that configuration b) is not allowed

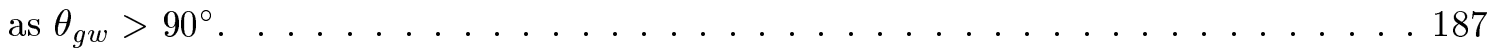

11.8 Pore-scale arrangements of fluid for water-wet, oil-wet, and mixed-wet corners. A thick line represents an oil-wet surface. Notice that oil layers sandwiched between water and gas are present for both the water-wet and mixed-wet media, leading to similar $k_{r o}$ at low $S_{o}$. In oil-wet media, oil is confined to the crevices, leading to a

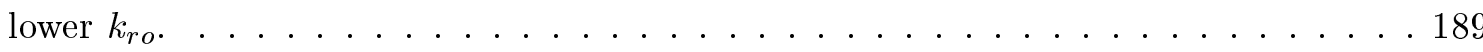

11.9 Comparison of relative permeabilities. (a) A spreading system $\left(\theta_{g o} \approx 0\right)$ : Octane in water-wet sand. (b) A nonspreading system : Decane in water-wet sand. (c) A nonwetting system : Water in oil-wet sand. (d) A mixed-wet system : Octane in mixed-wet sand. The solid lines in (a) and (d) show $k_{r o} \sim{S_{o}}^{2}$. The data, while consistent with this trend, only follows it approximately. . . . . . . . . . 190 


\section{Executive Summary}

This final report describes research carried out in the Department of Petroleum Engineering at Stanford University from September 1996 - May 2000 under a three-year grant from the Department of Energy on the "Prediction of Gas Injection Performance for Heterogeneous Reservoirs." The aim of this research project was an integrated study of the factors affecting gas injection, from the pore scale to the field scale, and involves theoretical analysis, laboratory experiments and numerical simulation. The proposal divided the work into four main areas: (1) Pore scale modeling of three phase flow in porous media; (2) Laboratory experiments and analysis of factors influencing gas injection performance at the core scale with an emphasis on the fundamentals of three phase flow; (3) Benchmark compositional simulations in heterogeneous media; and (4) Development of streamline-based reservoir simulation tools. The following chapters give a detailed account of our progress in these areas.

Chapters $2-4$ describe our development of streamline-based reservoir simulation tools. Chapter 2 introduces our three-dimensional streamline based reservoir simulator that can handle effects of gravity and changing well conditions for tracer flow, miscible flow and waterflooding. Chapter 3 extends the streamline methodology to compositional displacements and shows the streamline approach to be hundreds to thousands times faster than conventional grid-based methods. Chapter 4 reports a nested-gridding technique that accelerates streamline-based simulation still further and offers a direct alternative to upscaling.

Chapters 5-7 describe the development of method-of characteristics analytical solutions for the complex thermodynamics and flow of multicomponent gas/oil mixtures. Chapter 5 describes how to calculate the minimum miscibility pressure (MMP) or minimum miscibility enrichment (MME) rigoursly for reservoirs containing an arbitrary number of chemical components. Chapter 6 extends the computation of the MMP and MME for gas injection problems in which the gas also contains an arbitrary number of chemical components, and Chapter 7 develops an automatic procedure for finding the full 1D composition profile for gas injection below the MMP.

Chapters $8-11$ report advances in understanding the basics of three-phase flow through pore-scale modeling and core-scale experiments. Chapter 8 describes a three-phase pore-scale model which can compute macroscopic parameters, such as local oil recovery, relative permeability and capillary pressure from the basic pore-scale physical processes and fluid properties. Chapter 9 provides a framework for analyzing three-phase flow experiments. Chapter 10 describes threephase drainage experiments that were monitored using CT scanning resulting in measurements of three-phase relative permeabilities for fluids of varying spreading coefficients. Finally, Chapter 11 extends these measurements to systems of varying wettability and interprets the results in terms of the pore-scale physics unique to three-phase flow.

The work summarized in this report sets the stage for future work to combine better models of three-phase flow, analytical solutions of gas injection processes, and very fast streamline methods to create future physically-based reservoir simulation tools that are extremely efficient. 


\section{Introduction}

This final report describes research carried out in the Department of Petroleum Engineering at Stanford University from September 1996 - May 2000 under a three-year grant from the Department of Energy on the "Prediction of Gas Injection Performance for Heterogeneous Reservoirs." The research project was an integrated study of the physics and chemistry affecting gas injection, from the pore scale to the field scale, and involves theoretical analysis, laboratory experiments and numerical simulation. Specifically, advances were made on streamline-based simulation, analytical solutions to 1D compositional displacements, and modeling and experimental measurements of three-phase flow. Following are brief summaries of the research reported in each chapter.

Chapters 2, 3 and 4 describe our advances in streamline-based simulation. Chapter 2 introduces our three-dimensional streamline based reservoir simulator that can handle gravity and changing well conditions for tracer flow, miscible flow and waterflooding. Where comparison with conventional grid-based simulation is possible, the streamline method is hundreds to thousands of times faster and more accurate. These methods offer great promise for improving significantly our ability to predict flow in heterogeneous reservoirs at the field scale.

Chapter 3 extends the streamline methodology to compositional displacements where it is an attractive alternative to conventional grid-based methods. A general, compositional model is solved in one dimension along each streamline, accounting for phase behavior and volume change on mixing. The method is shown to be much faster and more accurate than conventional simulation. The streamline method is particularly well suited to study complex gas injection projects in highly heterogeneous media, where the combination of well placement, geological variability and phase behavior poses a particular challenge to finite-difference and finite-element codes.

Chapter 4 reports a nested-gridding technique that accelerates streamline-based simulation still further and offers a direct alternative to upscaling. Both conventional and streamline-based simulation approaches are limited by the memory and computer time requirements to solve for the pressure field. We propose a method where the pressure field is found approximately. First the pressure is computed on a coarse grid using averaged mobilities. This pressure solution is then used as a boundary condition for finding the pressures on the fine grid within each coarse grid block. The fluids are transported along the resulting streamlines, which reflect the fine grid details of the flow field. In this way, all small scale details of the flow are captured, with computations up to 10 times faster than streamline simulation on the full grid. Since the streamline method is itself orders of magnitude faster than grid-based approaches for displacement-type problems, the nested gridding approach offers huge speed advantages over conventional methods. Where traditional upscaling, with its inherent inaccuracies may have been required to perform a simulation on a fine scale reservoir description, this approach offers the possibility of running simulations directly on the fine grid.

A key feature of streamline methods is the use of one-dimensional (1D) solutions for flow along individual streamlines. In Chapters $2-4$, solutions along individual streamlines were computed numerically. Large additional speedups will be possible if fast methods for analytical solutions of the 1D flow problems can be found.

Chapters 5, 6 and 7 represent significant advances in our campaign to develop analytical solutions for the complex thermodynamics and flow of multicomponent gas/oil mixtures inherent in gas injection problems. In Chapter 5 we make use of the properties of solutions to the flow equations obtained by the method of characteristics. The solutions are used to calculate theoretically the minimum miscibility pressure (MMP) or minimum miscibility enrichment (MME) for a one or two component injection gas and a reservoir containing an arbitrary number of components. The 
calculation method is based on the idea that a series of key tie lines control the displacement behavior. Once the key tie lines are found, the MMP (or MME) is the pressure (or enrichment) at which one of the key tie lines has zero length. This approach is an efficient one that does not require that the full $1 \mathrm{D}$ solution be found.

Chapter 6 extends the computation of the MMP for gas injection problems in which the gas also contains an arbitrary number of chemical components. Injection, initial and crossover tielines are constructed for a general $n$ component system, and the tie-line that controls miscibility is identified. The predictions of this general and accurate method for obtaining the MMP compare favorably with experimental measurements and are shown to be superior in terms of speed and accuracy to other numerical methods.

Chapter 7 builds further on the method by developing an automatic procedure for finding the full 1D composition profile for gas injection below the MMP. The method is orders of magnitude faster than direct compositional simulation and suffers from no numerical dispersion. This work sets the stage for use of analytical 1D solutions in place of finite difference compositional solutions for slim tube displacements and in streamline compositional simulations.

Chapters 8-11 report advancements in understanding the basics of multiphase flow. Chapter 8 describes the results from a pore scale model of three phase flow in porous media. By coding pore scale displacement processes observed in micromodels and predicted from theoretical considerations, a numerical model may be developed that can compute macroscopic parameters, such as local oil recovery, relative permeability and capillary pressure from an understanding of the basic physics at the pore scale. We show that the three phase relative permeabilities depend on fluid properties, particularly spreading coefficient, and are extremely sensitive to the saturation path taken during the experiment. However, one simple idea emerges: for gas injection when the oil and water saturations are both low, the oil relative permeability, $k_{r o} \sim S_{o}^{2}$. These predictions are tested experimentally in chapters 10 and 11.

Chapter 9 provides a framework for analyzing three-phase flow experiments. Using data from Grader and O'Meara [76], we show how displacement paths can be calculated from the threephase relative permeabilities. This sets the stage for the three-phase experiments described in chapters 10 and 11. Chapter 10 provides a detailed description of the method we use to find three phase relative permeabilities. The CT scanner is used to image the saturation distribution as a function of distance and time along a core during a gravity drainage displacement. From this the relative permeabilities may be found by direct application of Darcy's law and conservation of mass, with no end effects or experimental artifacts. Relative permeabilities are measured that span more than six orders of magnitude, down to oil saturations as low as $S_{o}=0.01$. An extensive study is performed on sand-packs and a Berea sandstone to study gravity drainage in water-wet media. The effects of initial oil saturation and the oil spreading coefficient are discussed. The results of the experiments are explained and interpreted in terms of the arrangement of fluids at the pore scale.

Chapter 11 extends the measurements to systems of varying wettability. Here the wettability of a sand-pack is altered using a physically-based sequence of saturation changes that mimics the displacement processes observed in oil reservoirs. An initially water-filled and water-wet pack is flooded with a heavy crude oil/octane mixture. The pack is then left to age for several days. During this time, surface active components of the oil in direct contact with the solid surfaces of the pore space absorb to the surface and alter its wettability. Regions that remain water-filled or are protected by a thick wetting film of water remain water-wet. We then perform a series of three phase displacements and use CT scanning to measure the three phase relative permeabilities. The results are compared with measurements on water-wet, uniformly oil-wet and fractionally-wet media. We show that having a physically-based pattern of wettability makes a significant difference to the relative permeabilities, compared to a medium where surfaces are either uniformly water- or oil-wet. 
The work summarized in this report sets the stage for future work to combine better models of three-phase flow, analytical solutions of gas injection processes, and very fast streamline methods to create future reservoir simulation tools that are based on the physics of the displacements and are very efficient. The goal is to build physics-based techniques that give accurate high-resolution predictions of displacement processes at field scale. 


\title{
2. Development of a Streamline Based Reservoir Simulator
}

\author{
Rod Batycky, Marco Thiele and Martin Blunt
}

We present a new streamline-based simulator applicable to field scale flow. The method is three-dimensional (3D) and accounts for changing well conditions that result from infill drilling and well conversions, heterogeneity, mobility effects, and gravity effects. The key feature of the simulator is that fluid transport occurs on a streamline grid, rather than between the discrete gridblocks on which the pressure field is solved. The streamline grid dynamically changes as the mobility field and boundary conditions dictate. A general numerical solver moves the fluids forward in space and time along each streamline. Multiphase gravity effects are accounted for by an operator-splitting technique that also requires a numerical solver. Because fluid transport is decoupled from the underlying grid, the method is computationally efficient and very large time steps can be taken without loss in solution accuracy.

We present results of the streamline-based simulator applied to tracer, waterflooding, and first-contact miscible (FCM) displacements in two and three dimensions. Where possible, comparisons with conventional methods indicate that the streamline model minimizes numerical diffusion and is up to two orders of magnitude faster. We also demonstrate the efficiency of the method on a field scale, million-gridblock 36 -well waterflood that includes a pattern modification plan to improve oil recovery. Lastly, we present results of the method applied to the House Mountain waterflood in Canada.

\section{$2.1 \quad$ Introduction}

The use of streamlines and streamtubes to model convective displacements in heterogeneous media has been presented many times since the early works of Muskat[144, 145, 146], Fay \& Prats[61], and Higgins \& Leighton[90, 91, 92]. Important contributions to the field are also due to Parsons[169], Martin \& Wegner[132], Bommer \& Schechter[20], Lake et al.[123], Emanuel et al.[52], and Hewett \& Behrens[87, 86].

Streamline methods have recently resurfaced as a viable alternative to traditional finitedifference methods for large, heterogeneous, multiwell, multiphase simulations[184, 38, 206, 207, $205,18,14,11,10,22,88,173,204]$. The efficiency of the method has made it an ideal tool for ranking equiprobable reservoir images [208], rapid assessment of production strategies such as infill drilling and gas injection [51], computing upscaled component flux properties for compositional simulation [34], and integration with production data for reservoir characterization [213]. The method has also allowed for the solution of fine-scale models $\left(\mathcal{O}\left(10^{6}\right)\right.$ gridblocks) on standard computer resources, thus reducing the need for significant upscaling.

In this report we present advances on our previous work where we mapped analytical solutions along streamlines $[205,11]$. Although the streamline paths were updated periodically to account for changing mobility fields, the method could not account for changing well conditions or gravity - two key phenomena that must be modeled in general field scale simulations.

We account for these mechanisms by mapping one-dimensional (1D) numerical solutions along streamlines, as first proposed by Bommer \& Schechter[20]. In doing so, nonuniform initial

\footnotetext{
${ }^{1}$ The material in this chapter is published as "A 3D Field-Scale Streamline-Based Reservoir Simulator," $S P E$ Reservoir Engineering (1997) 11, 246-254.
} 
conditions that appear along recalcuated streamline paths, resulting from changing well and mobility conditions, can be moved forward in space and time correctly. Streamline paths are updated and the transport process repeated. The grid on which the pressure field is solved is effectively decoupled from the streamline grid used to transport fluids. There is no longer a global grid Courant-Friedrichs-Lewy (CFL) condition to restrict time step size. Furthermore, grid orientation and numerical diffusion effects are minimized. Finally, operator-splitting is used to account for gravity in multiphase flow [35, 74]. After moving fluids convectively along streamlines, fluids are then moved vertically along $1 \mathrm{D}$ gravity lines. Bratvedt et al.[22] presented a similar operator-splitting technique in the context of their front tracking method.

Our application of streamlines to field scale reservoir simulation is a combination of four existing ideas: (1) 3D streamlines [180], (2) updating the streamline paths to account for changing mobility field and well conditions [132, 184, 205], (3) numerical solutions along streamlines [20], and (4) including gravity effects in multiphase flow using operator-splitting [10, 22, 35, 74]. Using streamlines and gravity lines decouples the 3D transport problem into multiple 1D problems and leads to a very fast and accurate method applicable to a wide range of field conditions.

\subsection{The Streamline Method}

In this section we outline the streamline method. For a detailed discussion on how to trace the streamlines we refer the reader to the Appendix.

\subsubsection{Governing IMPES Equations}

The streamline method is an IMPES method. Ignoring capillary and dispersion effects, the governing equation for pressure $p$, for incompressible, multiphase flow is given by

$$
\nabla \cdot \overrightarrow{\vec{k}} \cdot\left(\lambda_{t} \nabla p+\lambda_{g} \nabla D\right)=0
$$

where $\mathrm{D}$ represents a depth below datum. The total mobility $\lambda_{t}$ and the total gravity mobility $\lambda_{g}$ are defined as

$$
\lambda_{t}=\sum_{j=1}^{n_{p}} \frac{k_{r j}}{\mu_{j}}, \quad \lambda_{g}=\sum_{j=1}^{n_{p}} \frac{k_{r j} \rho_{j} g}{\mu_{j}} .
$$

$k_{r j}$ is the relative permeability of phase $j, \mu_{j}$ is the phase viscosity, $\rho_{j}$ is the phase density, $g$ is the gravity acceleration constant, and $n_{p}$ is the number of phases present. We also require a material balance equation for each phase $j[122]$,

$$
\phi \frac{\partial S_{j}}{\partial t}+\vec{u}_{t} \cdot \nabla f_{j}+\nabla \cdot \vec{G}_{j}=0
$$

The total velocity $\vec{u}_{t}$ is derived from the 3D solution to the pressure field (Eq. 2.1) and application of Darcy's Law. The phase fractional flow is given by

$$
f_{j}=\frac{k_{r j} / \mu_{j}}{\sum_{i=1}^{n_{p}} k_{r i} / \mu_{i}},
$$

and the phase velocity due to gravity effects is given by

$$
\vec{G}_{j}=\overrightarrow{\vec{k}} \cdot g f_{j} \nabla D \sum_{i=1}^{n_{p}} k_{r i}\left(\rho_{i}-\rho_{j}\right) / \mu_{i} .
$$

Eqs. 2.1 and 2.3 form the IMPES set of equations in the formulation of the streamline simulator. We will confine our discussion to the solution of these equations for two-phase flow. 


\subsubsection{Coordinate Transform}

In a conventional IMPES finite-difference simulator Eq. 2.3 is solved in its full 3D form using the previously calculated pressure field. In the streamline method, we transform the 3D equation into multiple 1D equations that are solved along streamlines.

Streamlines are launched from gridblock faces containing injectors. As the streamlines are traced from injectors to producers we determine the time-of-flight along the streamline, which is defined as $[38,180]$

$$
\tau=\int_{0}^{s} \frac{\phi}{\left|\vec{u}_{t}(\zeta)\right|} d \zeta
$$

and gives the time required to reach a point $s$ on the streamline based on the total velocity $\vec{u}_{t}(\zeta)$ along the streamline. The permeability, porosity, and total mobility effects of the 3D Cartesian domain are incorporated along a streamline via the $\tau$ coordinate.

To determine the coordinate transform, we rewrite Eq. 2.6 as

$$
\frac{\partial \tau}{\partial \zeta}=\frac{\phi}{\left|\vec{u}_{t}\right|}
$$

which can further be rewritten as

$$
\left|\vec{u}_{t}\right| \frac{\partial}{\partial \zeta} \equiv \vec{u}_{t} \cdot \nabla=\phi \frac{\partial}{\partial \tau}
$$

Substituting Eq. 2.8 into Eq. 2.3 gives

$$
\frac{\partial S_{j}}{\partial t}+\frac{\partial f_{j}}{\partial \tau}+\frac{1}{\phi} \nabla \cdot \vec{G}_{j}=0
$$

Eq. 2.9 is the governing pseudo-1D material balance equation for phase $j$ along a streamline coordinate. The equation is pseudo-1D since the gravity term is typically not aligned along the direction of a streamline. To solve Eq. 2.9 we split the equation into two parts based on operator-splitting as outlined by Glimm et al.[74], Colella et al.[35], and Bratvedt et al.[22]. First, a convective step along streamlines is taken governed by

$$
\frac{\partial S_{j}^{c}}{\partial t}+\frac{\partial f_{j}}{\partial \tau}=0
$$

to construct an intermediate saturation distribution $S_{j}^{c}$. Then, a gravity step is taken along gravity lines using

$$
\frac{\partial S_{j}}{\partial t}+\frac{1}{\phi} \frac{\partial G_{j}}{\partial z}=0
$$

with $S_{j}^{c}$ as the initial condition to construct $S_{j}$, and $G_{j}=\left|\vec{G}_{j}\right|$. For simplicity, we have assumed that the $z$-coordinate direction is aligned with the gravity lines.

\section{D Numerical Solvers}

One-dimensional numerical solvers are used to solve Eqs. 2.10 and 2.11. Each solver is completely decoupled from the rest of the simulator. Here we have chosen to solve Eq. 2.9 but in fact any equation with the desired physics written in 1D can be used. For example, this method has been extended to compositional displacements (see Chapter 3) [204].

For cases presented here, Eq. 2.10 is solved numerically using a single-point upstream (SPU) weighting scheme explicit in time. By discretizing in $\tau$ space, this leads to a natural refinement in 1D where flow velocities are high and reduced resolution where flow velocities are low. To retain 
accuracy within the numerical solver the irregular spaced $\tau$-grid is converted to a regular spaced $\tau$-grid. Time stepping for the SPU scheme is controlled within the solver using the optimal local CFL constraint particular to a given streamline such that the fastest front is always moved one $\tau$ node per local time interval $\left(\Delta t_{1 D}\right)$. The ability to honor the local CFL constraint minimizes numerical diffusion.

For the gravity solver, Eq. 2.11 is discretized in space limited to the same vertical resolution of the underlying grid on which the pressure field is defined. Eq. 2.11 is solved using an explicit upstream weighting method outlined by Sammon [188]. An additional advantage of decoupling the gravity step in this way is that Eq. 2.11 is only solved in flow regions where gravity effects are important. For example, in locations where fluids are completely segregated, Eq. 2.11 will not be solved since $\partial G / \partial z=0$.

\section{Time Stepping}

In field scale displacements the streamline paths change with time due to the changing mobility field and/or changing boundary conditions. Thus the velocity field is updated periodically in accordance with these changes, by solving for the pressure.

To move the 3D solution forward in time globally by $\Delta t$ from $t^{n}$ to $t^{n+1}=t^{n}+\Delta t$ we use the following algorithm:

1. At the start of a new time step, $t^{n+1}$, solve for pressure using Eq. 2.1. We solve Eq. 2.1 using a standard seven-point finite difference scheme, with no-flow boundary conditions over the surface of the domain and specified pressure or rate at the wells. The resulting linear set of equations is solved using a multigrid method [119].

2. Apply Darcy's Law to determine the total velocity at gridblock faces of the 3D Cartesian domain.

3. Trace streamlines from injectors to producers as outlined in the Appendix. For each streamline we do the following: (a) While tracing a streamline, the current saturation information from each gridblock that the streamline passes through is recorded. In this manner, a profile of saturation versus $\tau$ is generated for the new streamline. (b) Move the saturations forward $\Delta t$ by solving Eq. 2.10 numerically in 1D. This will involve several timesteps within the numerical solver since $\Delta t \gg \Delta t_{1 D}$. (c) Map the new saturation profile back to the original streamline path and onto the underlying grid.

4. Average all the streamline properties within each gridblock of the $3 \mathrm{D}$ domain to determine the saturation distribution at $t^{n+1}$.

5. If $G_{j} \neq 0$ include a gravity step that traces gravity lines from the top of the domain to the bottom of the domain along $\vec{g}$. For each gravity line we do the following: (a) The saturation distribution calculated in the convective step as a function of $z$ is traced along a gravity line. (b) The saturations are moved forward by $\Delta t$ using Eq. 2.11. (c) Map the new saturation profile back to the original gravity line and onto the underlying grid.

6. If $G_{j} \neq 0$ average all gravity line properties within each gridblock of the 3D domain to determine the final saturation distribution at $t^{n+1}$.

7. Return to Step 1.

In the above time stepping scheme, for tracer displacements with fixed boundary conditions the streamline paths do not change with time. The pressure field is only calculated once. $\Delta t$ represents a global convective time step between remappings. For nonlinear displacements (changing streamline paths), each global time step includes a pressure solve and a remapping step. 


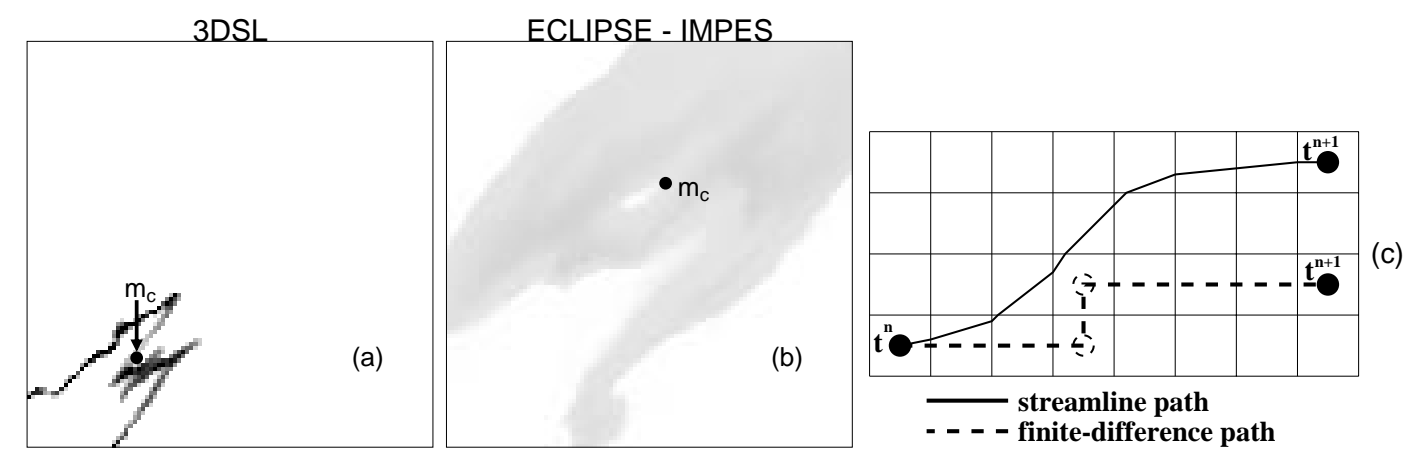

Figure 2.1: Distribution of a tracer slug using (a) the streamline method and (b) ECLIPSE-IMPES. $m_{c}$ is the center of mass of the tracer slug. (c) Conceptual picture illustrating the final position of a fluid element at the end of time interval $\Delta t$ by transporting along a streamline vs. the underlying Cartesian grid.

\subsection{Tracer Displacements}

In our first example, a small slug of tracer is injected into a $100 \times 100$ heterogeneous quarterfive spot model. The slug distribution at $t_{D}=0.07$ is shown in Figure 2.1 for the streamline simulator (3DSL) and ECLIPSE-IMPES[101] with a SPU scheme. This comparison demonstrates how conventional grid-based methods can suffer from large amounts of numerical diffusion and move fluid in the wrong direction. In contrast, the streamline method automatically captures the correct flow direction and moves fluid with minimal numerical diffusion. Note the difference in the location of the center of mass $m_{c}$ of the slug predicted by the two methods.

Figure 2.1c illustrates conceptually the difference between the two methods. The efficiency of the streamline method is a result of being able to take large global time steps along streamline paths. The CFL constraints of the underlying grid have been decoupled from transport and there is no limit to the size of $\Delta t$. For a conventional method, because transport is restricted to Cartesian directions, small global time steps must be taken but will not guarantee the same path as a streamline path. For this example, the streamline method required 2 time steps to move the slug profile in Figure 2.1 whereas ECLIPSE required over 900 time steps.

For both the streamline simulator and conventional methods, saturation information is only known at the scale of a single gridblock. Each time we map the streamline saturation information to the underlying grid we mix all of the streamline saturations within each gridblock. For the numerical solutions, these mixed properties are then picked up at the next time step and moved forward. In this manner streamlines communicate with each other within each gridblock after a global time step. The number of remappings will influence the level of mixing present in the solution. We will study this mixing next and compare results with those from mapping an analytical solution along streamlines [205, 11], which for tracer displacements give exact results in the case of no physical dispersion or diffusion.

For the numerical solutions, $G_{j}=0$ in Eq. 2.11 and $S_{j}=f_{j}=C$, where $C$ is the tracer concentration, in Eq. 2.10. Figure 2.2 shows a comparison of tracer flow results in a heterogeneous $250 \times 100$ medium for the exact solution (mapping the analytical $1 \mathrm{D}$ tracer profile) and the numerical mapping technique using both a SPU scheme and a high-order TVD scheme [17] to solve Eq. 2.10. The use of TVD schemes for transport equations along streamlines is discussed in detail elsewhere $[173,204]$. Taking the entire time step $\Delta t$ within the 1D solver and mapping only once to the underlying grid (Figure 2.2b,d) gives results almost identical to the reference solution. There is no difference between using a SPU or a TVD scheme. This is because we move fluid at the 


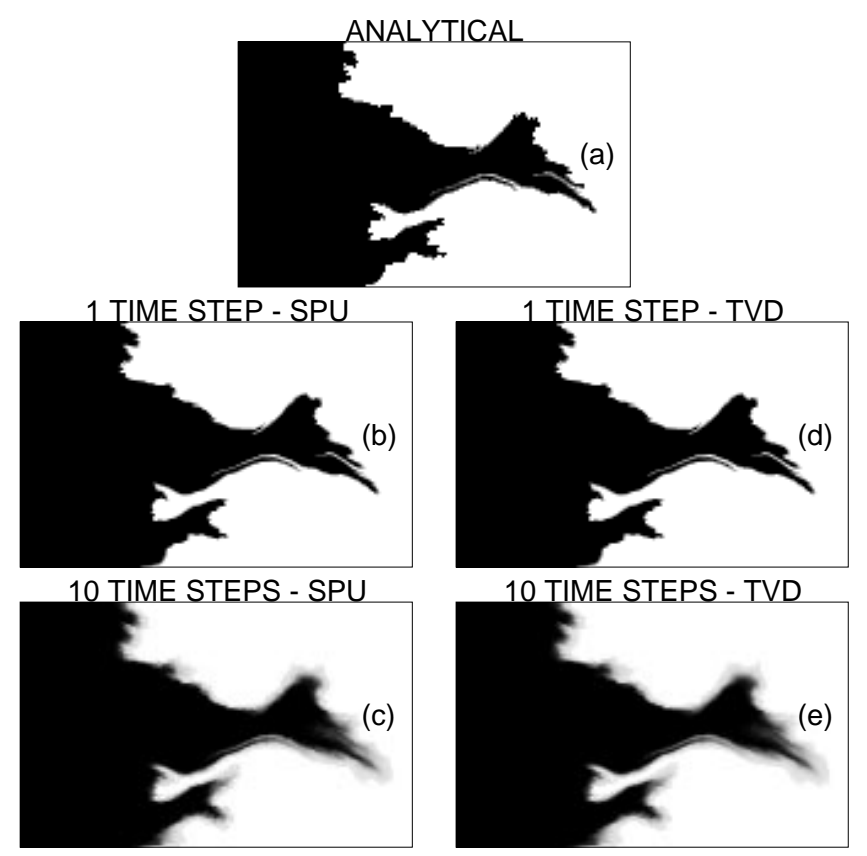

Figure 2.2: Comparison of tracer profiles in a $250 \times 100$ heterogeneous domain using the streamline simulator: (a) mapping an analytical tracer solution along streamlines, (b) mapping a SPU (numerical) solution with 1 remapping step, (c) mapping a SPU solution with 10 remapping steps, (d) mapping a TVD (numerical) solution with 1 remapping step, (e) mapping a TVD solution with 10 remapping steps.

optimum CFL number along a streamline, such that the fastest front moves one $\tau$ interval per local time step $\Delta t_{1 D}$. Under this condition a SPU scheme will not suffer from numerical diffusion and is equivalent to a high-order scheme. We see similar behavior for waterflood displacements. Thus, for the remaining cases in this report we use the SPU scheme. However, for more complex 1D systems, where the front speed is not known analytically - such as compositional displacements - a TVD scheme can offer improved accuracy [204].

If the convective time step is reduced by a factor of 10 , then 10 time steps are taken to reach $t_{D}=0.3$. There are 10 remappings to the underlying grid resulting in the saturation profiles shown in Figure 2.2c,e. Again there is no difference between the SPU or TVD scheme. However, there is more diffusion than in Figure 2.2b,d, and it is due to increased mixing of streamline properties at the gridblock scale.

As we show later, for nonlinear displacements where the flow field changes with time, the streamline method requires only 10's of time steps per pore volume injected (PVI) to produce converged results. The degree of mixing is approximately that shown in Figure 2.2c,e and is smaller than numerical diffusion in conventional methods. This is important since numerical diffusion can feed back into errors in the flow field calculation, resulting in compounding errors in recovery predictions.

\subsection{First-Contact Miscible Displacements}

For first-contact miscible displacements we use Eq. 2.10 with $S_{j}=f_{j}=C$, where $C$ is the concentration of the miscible injectant. Effective phase viscosities and densities are calculated using the Todd \& Longstaff method [209]. For all cases shown here the Todd \& Longstaff mixing 

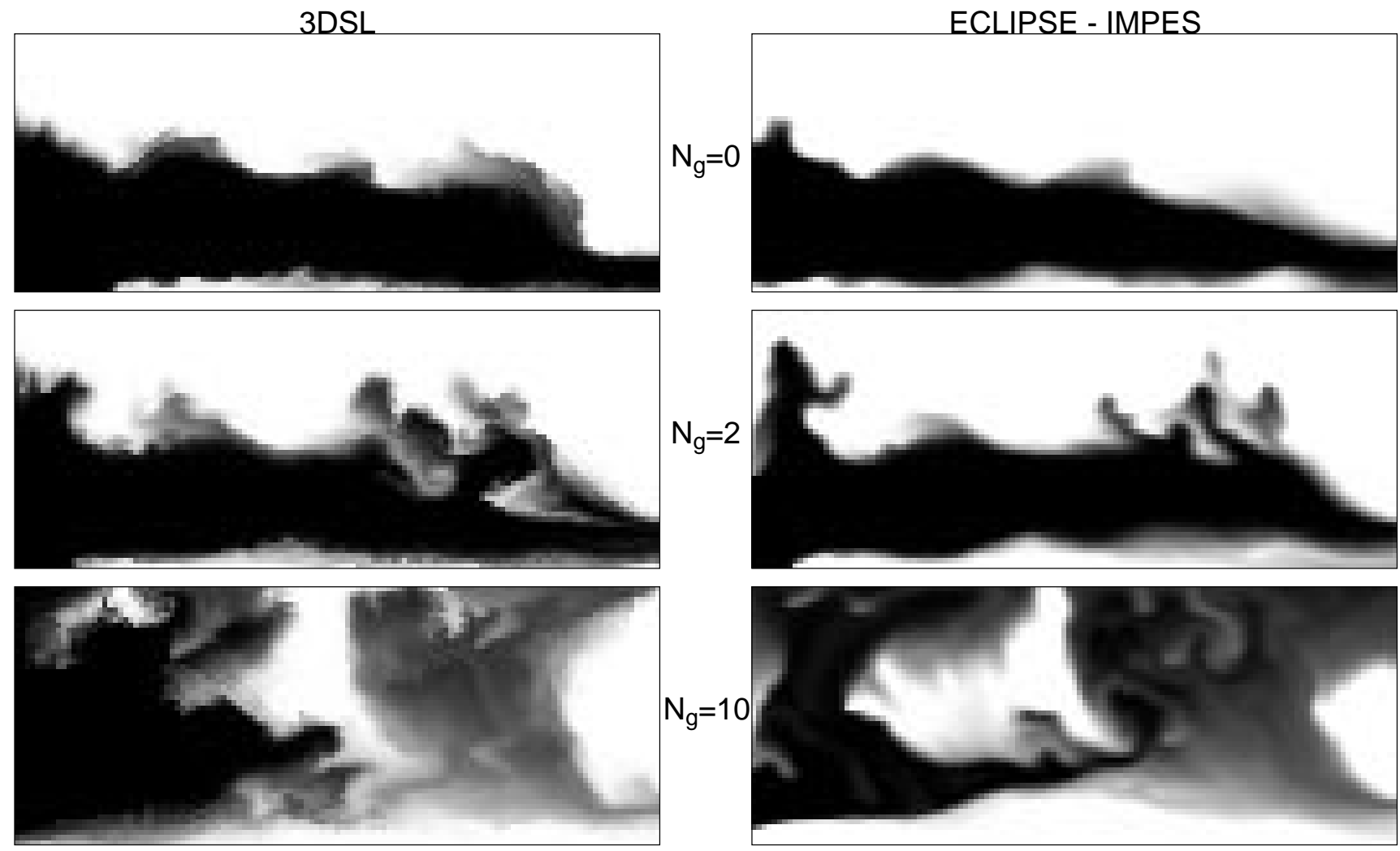

Figure 2.3: Comparison of FCM solvent profiles between 3DSL and ECLIPSE at $t_{D}=0.6$ in a $125 \times 50$ heterogeneous model at three gravity numbers. Injection is into the lower 10 left gridblocks and production is from the lower 10 right gridblocks.

parameter is assumed to be $\omega=1$. This implies that fluid properties between the miscible phases are completely mixed at the gridblock scale. As a result the effective phase densities within any gridblock are equal $\left(G_{j}=0\right)$ and only Eq. 2.10 governs transport.

We define a gravity number as [203]

$$
N_{g}=\frac{\bar{k}_{V} \Delta \rho g L^{2}}{\bar{k}_{H}\left(\overline{\Delta p}_{H}\right) h},
$$

where $\bar{k}_{V}$ and $\bar{k}_{H}$ are average vertical and horizontal permeabilities respectively, $\Delta \rho$ is the pure fluid density difference, $\overline{\Delta p}_{H}$ is the pressure drop in the horizontal direction, $L$ is the distance between wells, and $h$ is the model height.

Shown in Figure 2.3 are the solvent profiles for a $125 \times 50$ heterogeneous system at $t_{D}=0.6$ for the streamline method at three different gravity numbers. Injection is in the lower ten gridblocks on the left and production is from the lower ten gridblocks on the right. The pure fluid viscosity ratio was kept constant at 10, and phase densities were altered to change the gravity number. Clearly, the streamline simulator (3DSL) can model gravity dominated FCM displacements as seen by the increased amount of solvent rising in the model as gravity forces are increased. For comparison, ECLIPSE-IMPES two-point upstream weighting results with the same parameters are also shown in Figure 2.3. The solvent distributions are similar between the two methods, although there appears to be more detail in the 3DSL pictures. A summary of oil recovery is shown in Figure 2.4 for each gravity number. In general, ECLIPSE under predicts recovery compared to the streamline method. There appears to be more numerical diffusion within ECLIPSE which causes increased 


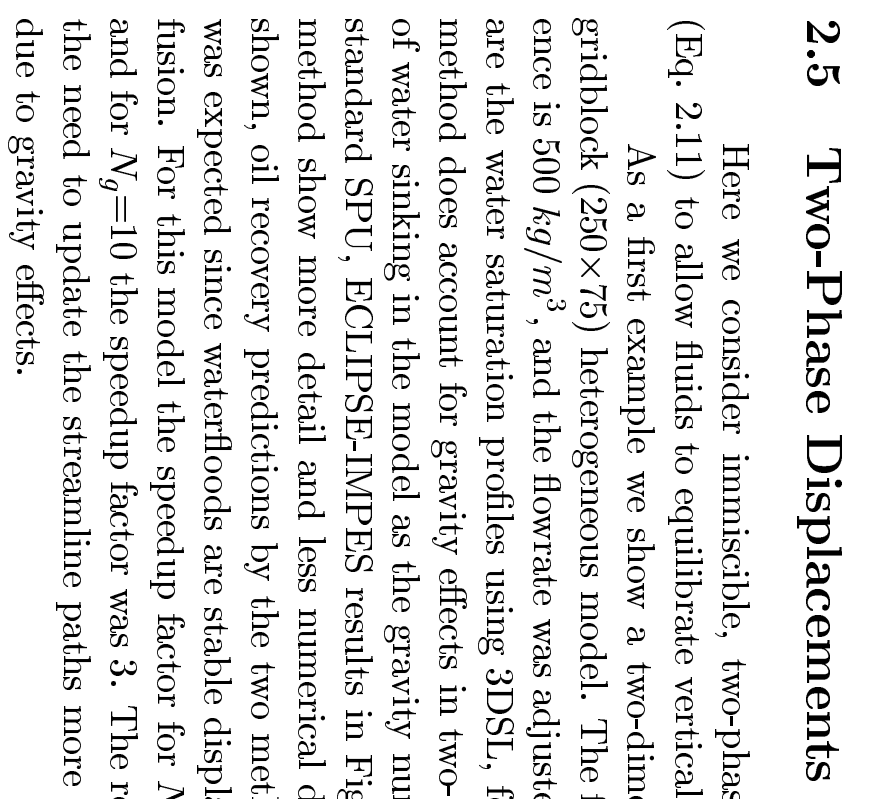

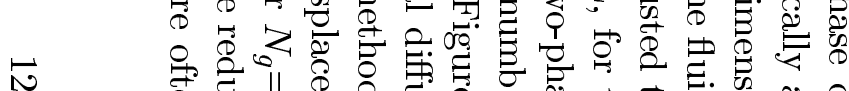

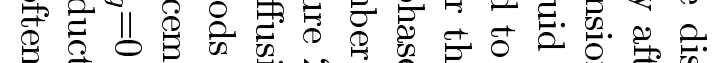

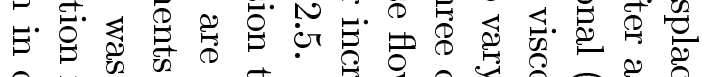

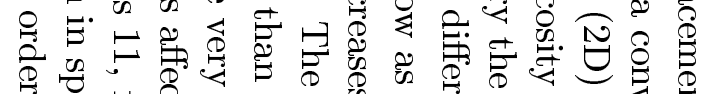

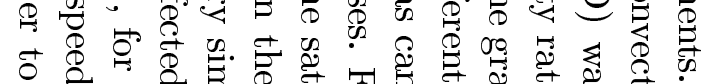

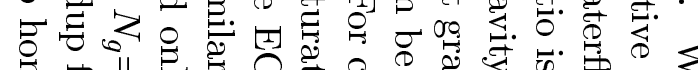

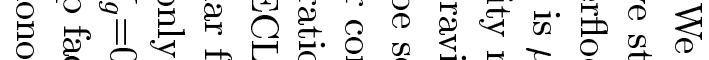

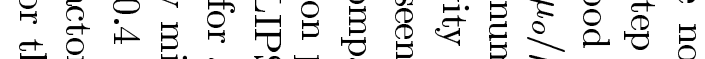

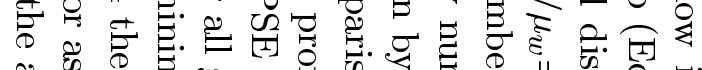

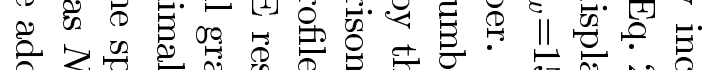
窟.

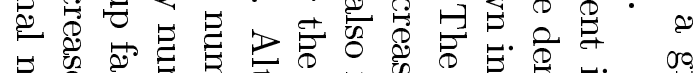

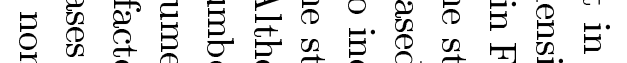
:

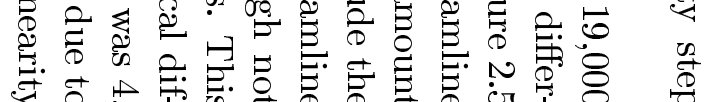

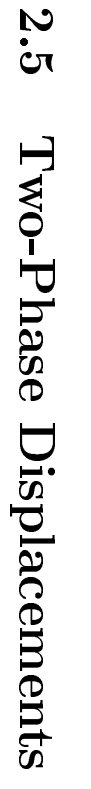

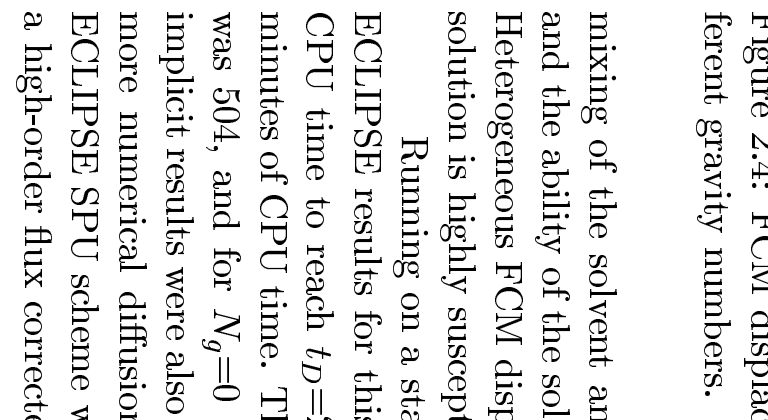

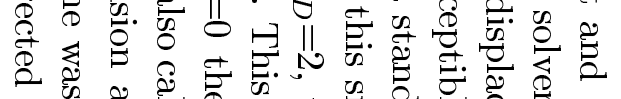

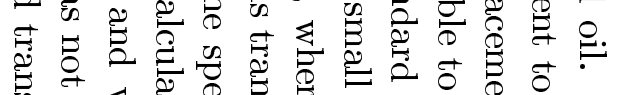

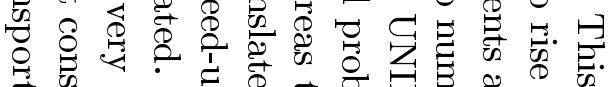

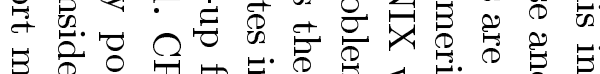

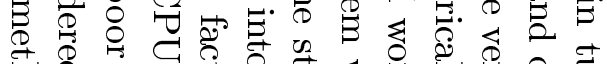
5

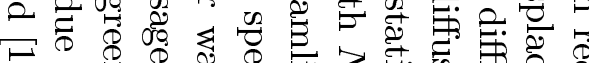

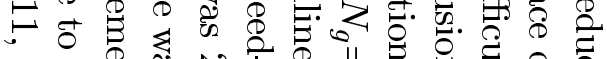

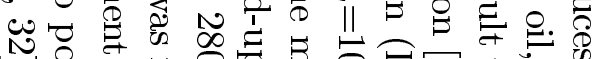
更. 若.

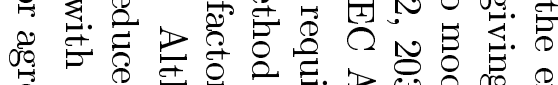

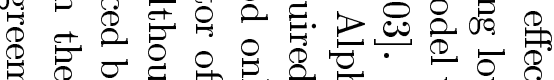

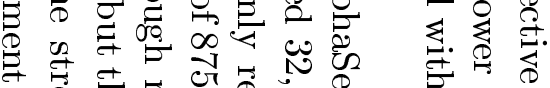

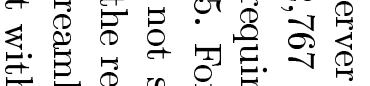

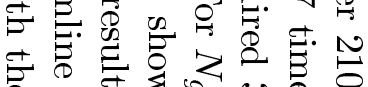
का क्य

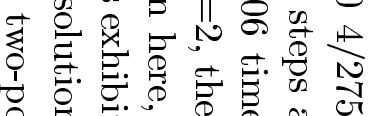
.

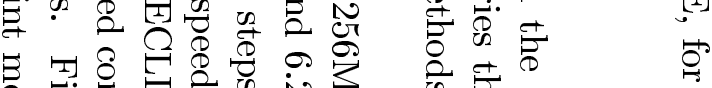

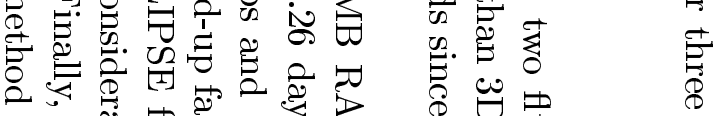

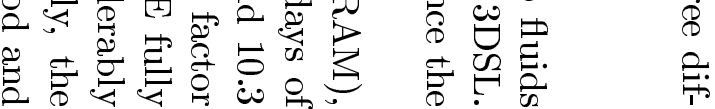

Dimensionless Recovery, $\mathrm{E}_{\mathrm{R}}$

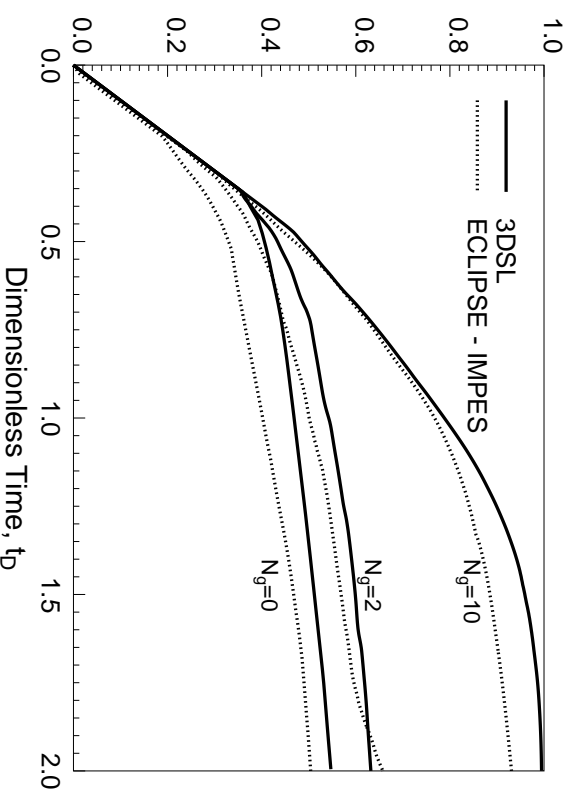



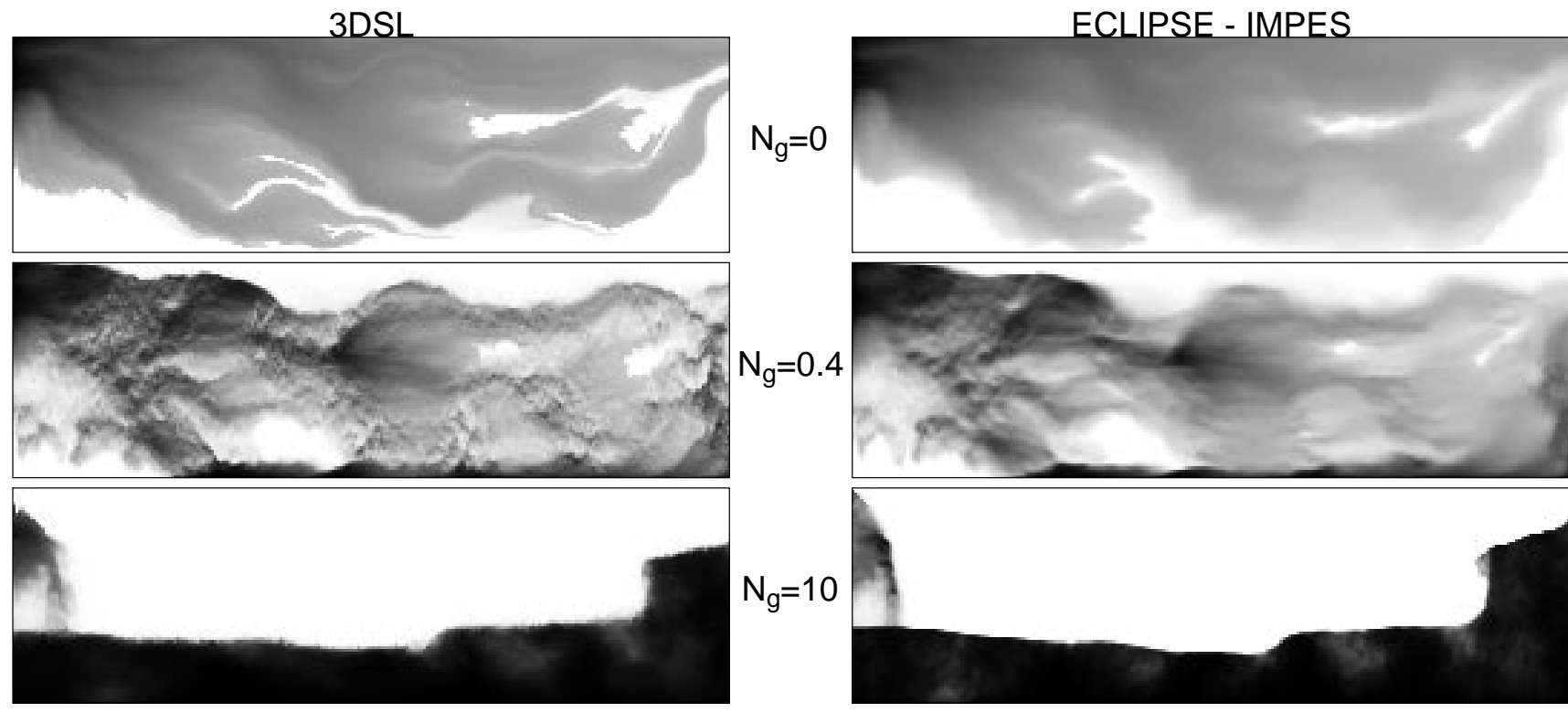

$\mathrm{N}_{\mathrm{g}}=10$

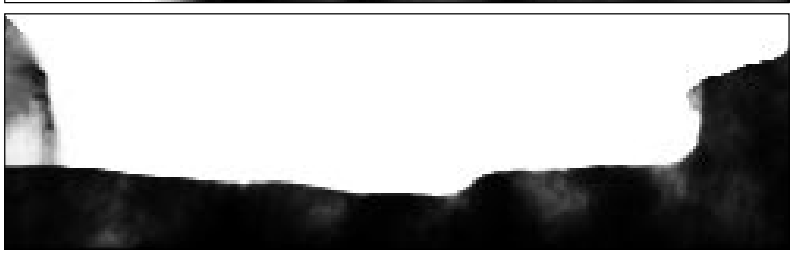

Figure 2.5: Comparison of water saturation profiles between 3DSL and ECLIPSE at $t_{D}=0.4$ in a $250 \times 75$ heterogeneous model at three gravity numbers. Injection is into the upper 10 left gridblocks and production is from the upper 10 right gridblocks.

As a second example we solve a 100,000 gridblock $(100 \times 100 \times 10) 5$-spot waterflood with and without gravity. An injection well is located in the lower two central gridblocks, and a production well is located in the upper two gridblocks in each corner of the model. The water distributions with and without gravity using 3DSL are shown in Figure 2.6. We compare the streamline results against the ECLIPSE-IMPES SPU method. Figure 2.7 shows that the oil recovery predicted by the two methods is similar, although there are some small differences for the $N_{g}=1$ case. We attribute these differences to grid orientation effects in ECLIPSE and the ability of 3DSL to better model the water cone to each producer. For the case $N_{g}=0$, the streamline model required 50 minutes run time while the equivalent ECLIPSE model required 101 hours run time, a speedup factor of 121. For the case $N_{g}=1.0$, the streamline model required 5.4 hours run time while ECLIPSE required 297 hours (12.4 days) run time, a speedup factor of 55 .

\subsection{Million Gridblock Waterflood}

A major limitation of previous streamline models was the inability to account for changing well conditions. Mapping numerical solutions along the streamlines removes this deficiency and allows for shut-in of wells, conversion of producers to injectors, and infill drilling.

We illustrate this flexibility with a field scale problem, a 1.16 million gridblock $(220 \times 220 \times 24)$ waterflood model containing 9 producers and 9 injectors in 5-spot patterns. The permeability field, generated using sequential Gaussian simulation [41], is highly heterogeneous with a permeability trend aligned diagonal to the 5 -spots. The correlation length is $\lambda_{c}=0.25$ in the on-trend direction, $\lambda_{c}=0.03$ in the off-trend direction, and $\lambda_{c}=0.17$ in the vertical direction. The producers are completed in the upper 12 gridblocks and the injectors are completed in the lower 12 gridblocks. At $t_{D}=0.4$ all the producers are recompleted in the lower portion of the model and converted to injectors, while 16 additional infill producers are added to give a line-drive waterflood.

The million gridblock model was run using 3DSL on a standard workstation and required 


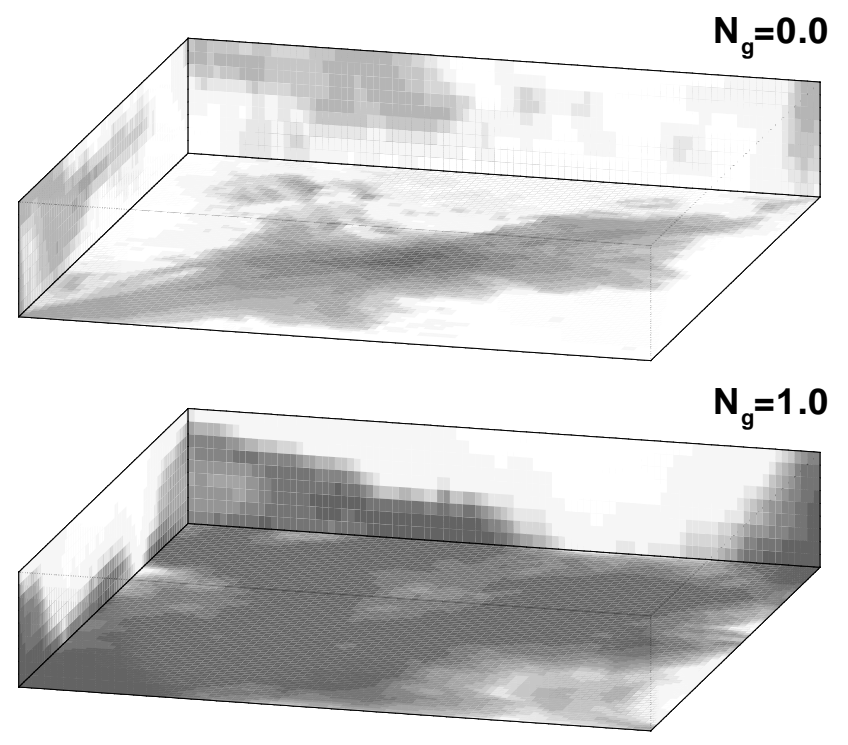

Figure 2.6: Water saturations predicted using 3DSL with and without gravity (100,000 gridblocks). Injection is in the central bottom two gridblocks, production is from the top two gridblocks of each corner.

50 hours for the base case recovery curve, and 40 hours for the incremental results. The base case and incremental recovery due to the pattern modifications are shown in Figure 2.8. We could not run this model with ECLIPSE using our current computer resources. To compare with ECLIPSE, the model was upscaled by a factor of 16 to 72,000 gridblocks $(110 \times 110 \times 6)$ using geometric averaging of absolute permeability. Relative permeabilities were not altered for the coarse scale model. The oil recovery results for ECLIPSE-IMPES SPU are also shown in Figure 2.8. For this model the ECLIPSE base case recovery curve required 55 hours run time, while the incremental results required 13 hours run time. It is worth noting that an ECLIPSE fully implicit solution for the base case model required 120 hours run time due to time step convergence problems for this size model. Included for reference are 3DSL upscaled results, which required 28 minutes run time for the base case and 22 minutes run time for the incremental case.

As seen in Figure 2.8, incremental oil recovery due to infill drilling is underestimated in the upscaled model. This is because upscaling leads to a larger over prediction of oil recovery for the base case 5-spot model, than for the line-drive model. We attribute this to the difference in the inter-well permeability correlation lengths, which are $50 \%$ smaller in the line-drive pattern than the 5-spot pattern. This comparison highlights two points: (1) the ECLIPSE base case model was 16 times smaller than the 3DSL model, yet both required approximately the same run time, and (2) ignoring fine-scale heterogeneity can lead to an over optimistic prediction of field performance.

\subsection{Convergence}

A key issue in the streamline method is how large a global time step $\Delta t$ can be allowed between pressure solves before the streamline paths require updating. For a conventional IMPES scheme the maximum time step size is linked to the grid CFL constraint dictated by the largest flow velocity anywhere in the domain (typically near a well). Small time steps result, causing fronts in most of the domain to move at far less than the optimal one gridblock per time step giving rise 


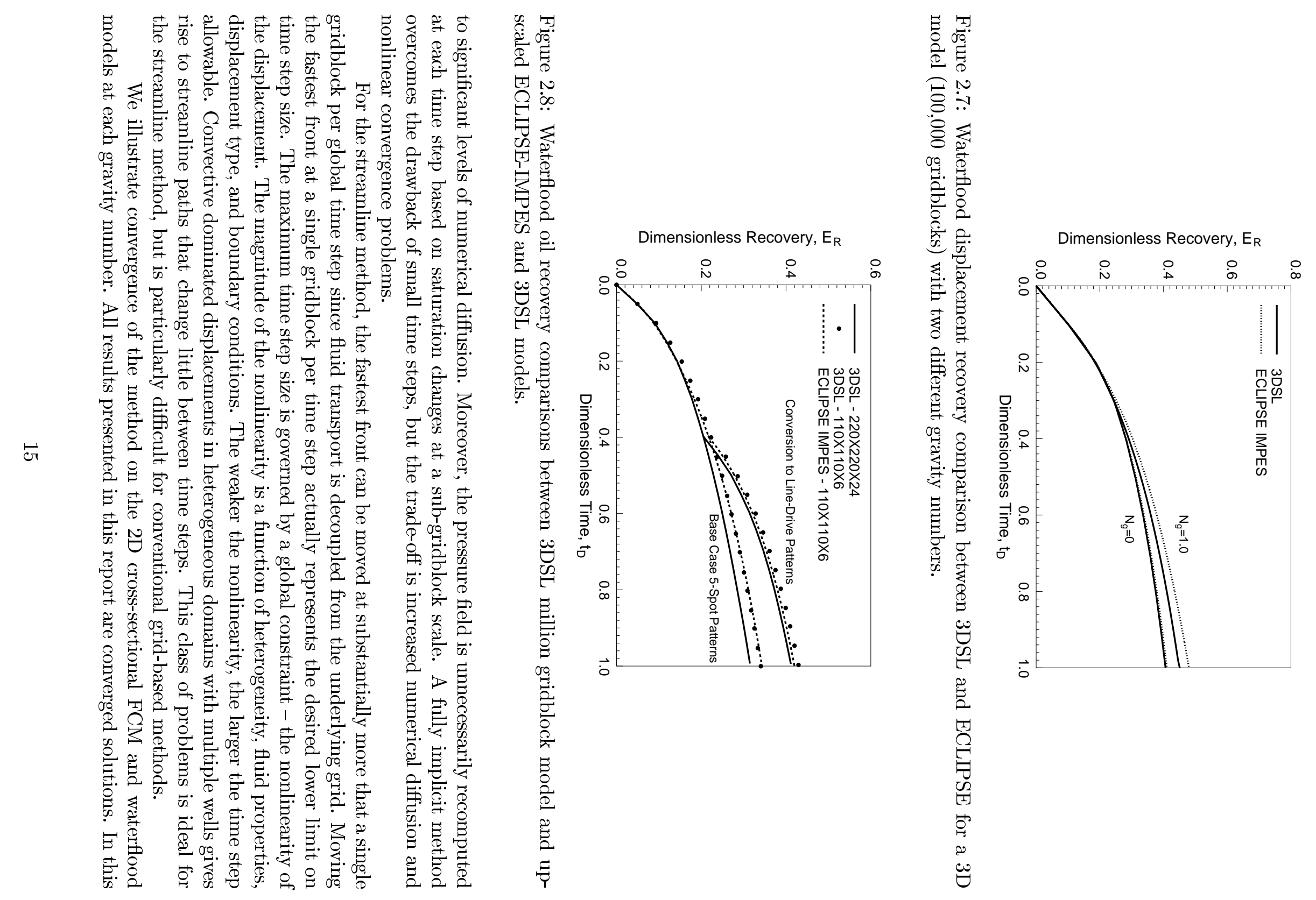




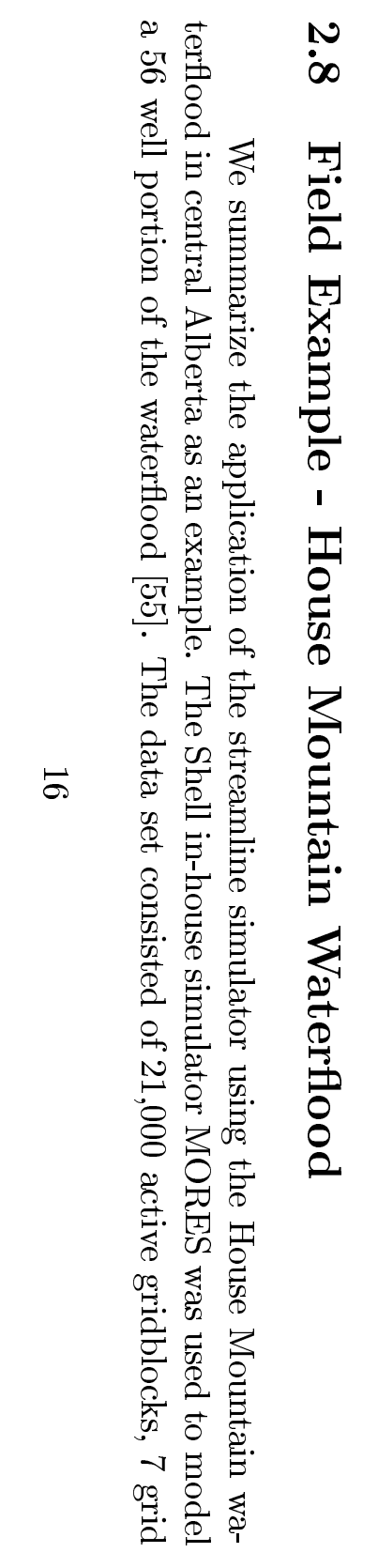

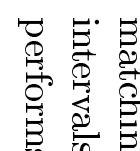

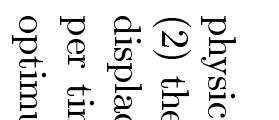

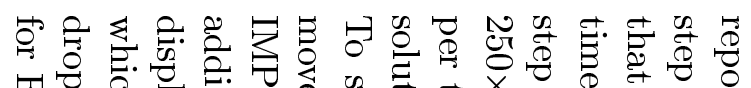

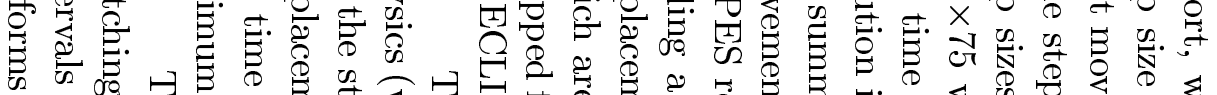
等

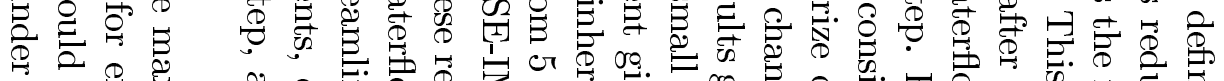

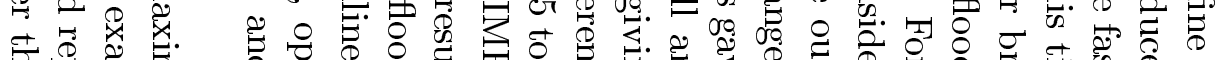

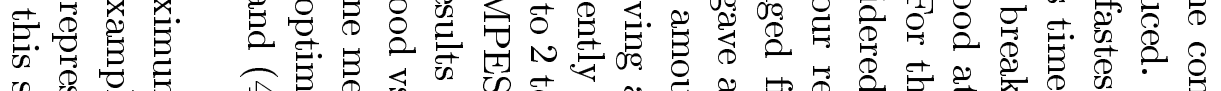

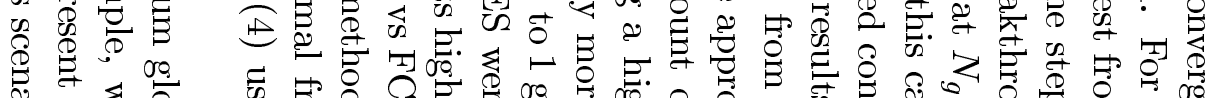

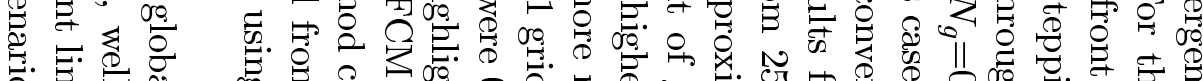

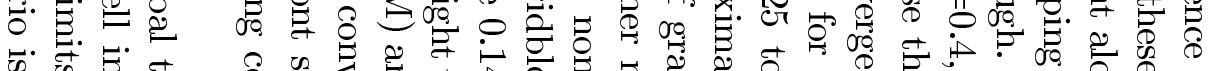

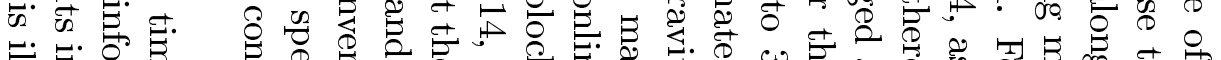

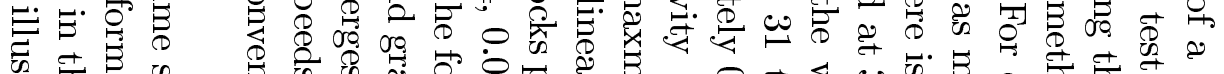

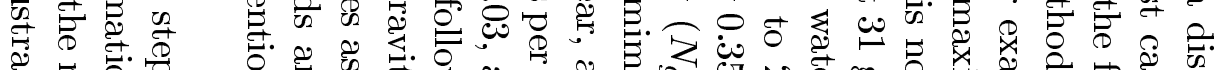
क

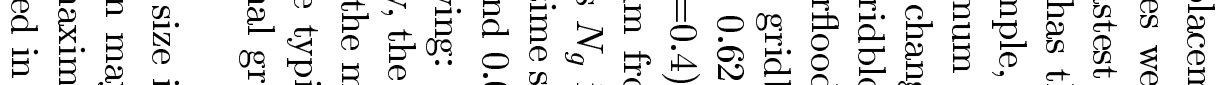

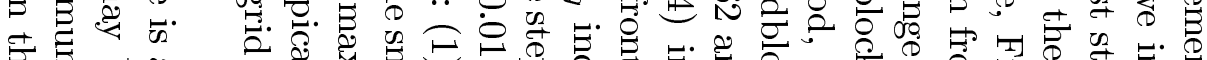

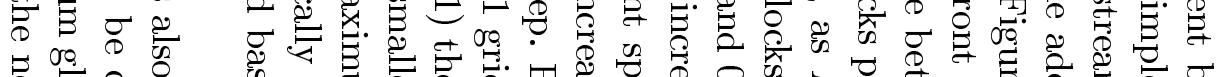

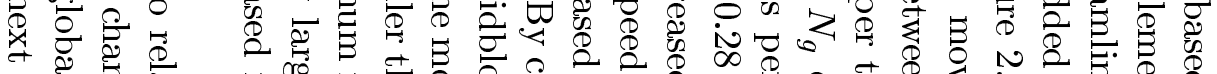

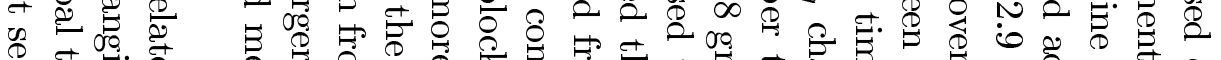

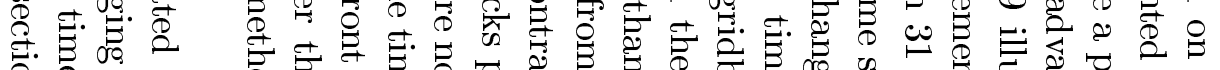

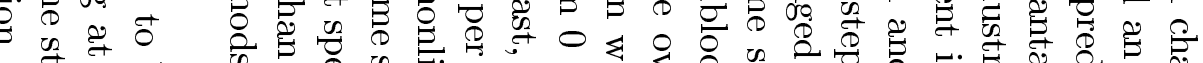
क्षृ क.

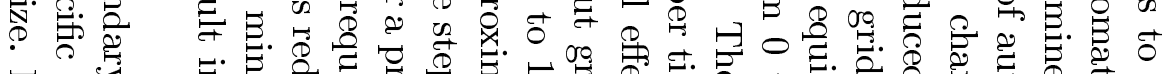
它 吉部.

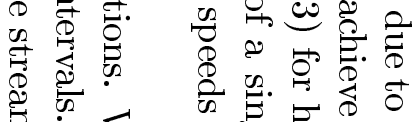

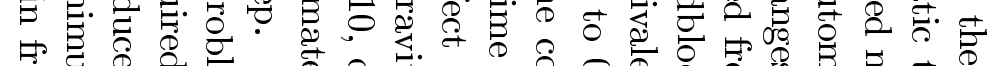

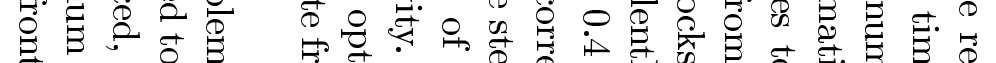

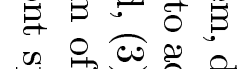

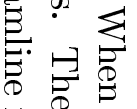

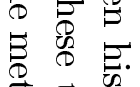

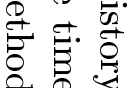
की

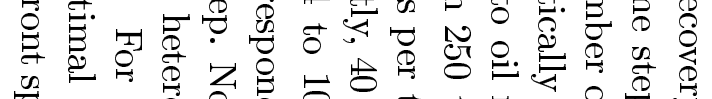

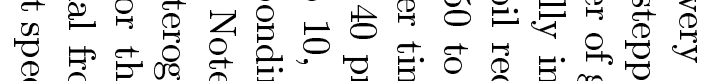

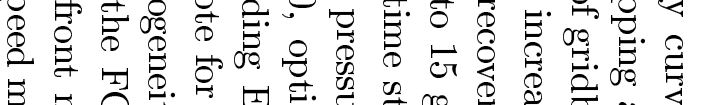

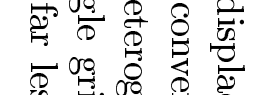

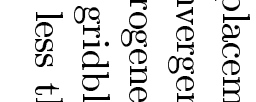

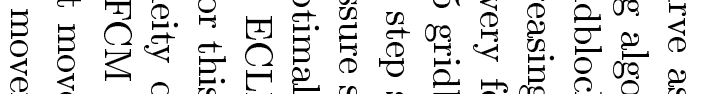

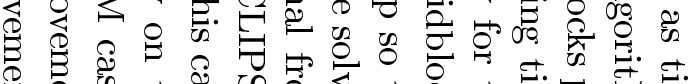

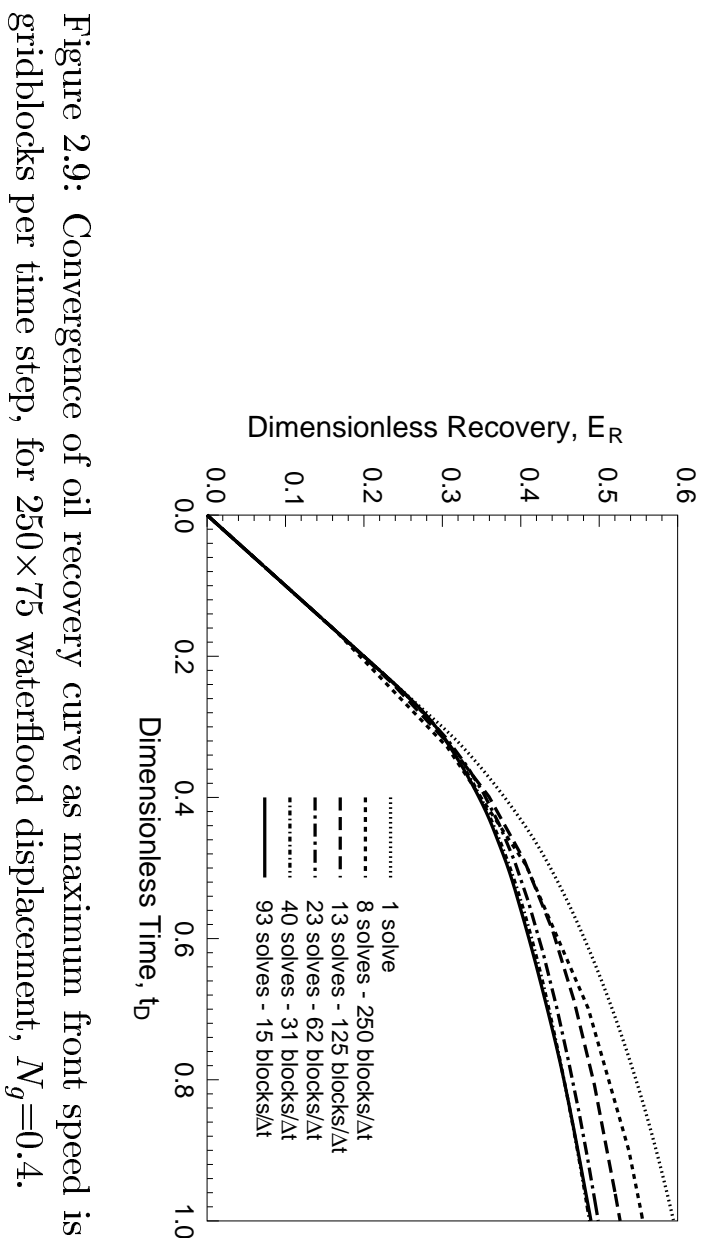



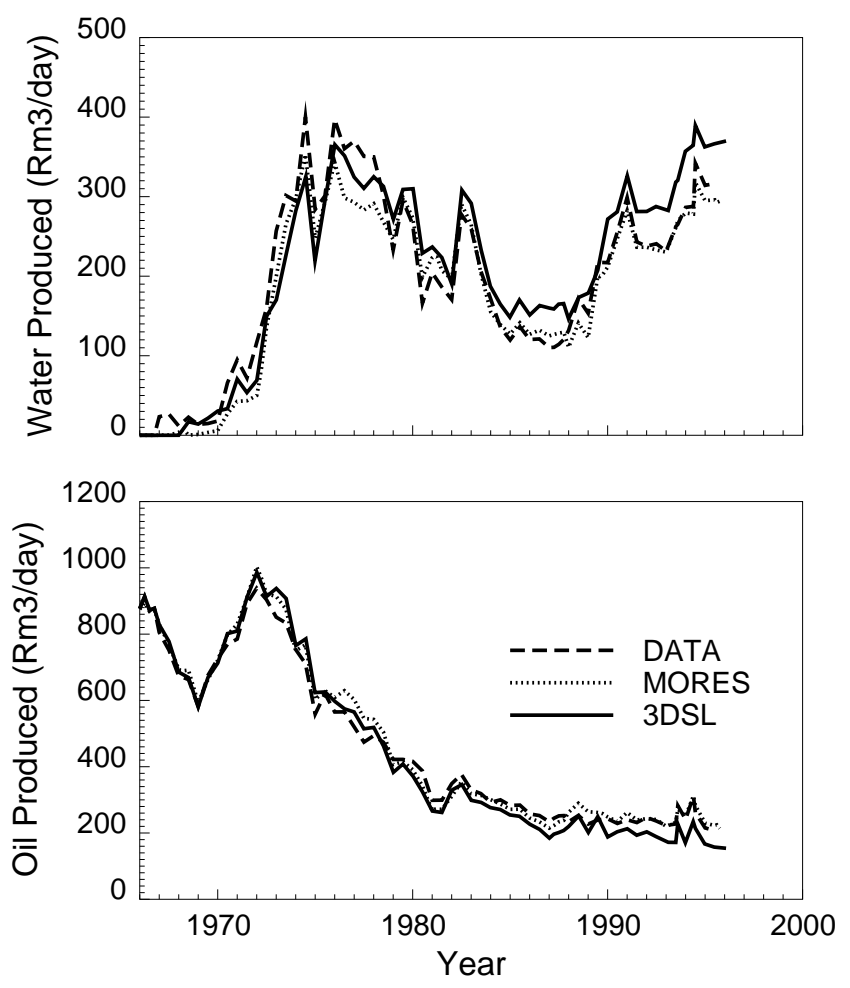

Figure 2.10: Comparison of historical production rate data with history matches for the streamline simulator (3DSL) and a conventional implicit simulator (MORES).

layers, three main rock types, and included vertical barriers in the southern half of the model. Production data consisted of 30 years of history at 6 month intervals with standard shut-ins and start-ups, 14 well abandonments, and 2 infill and 2 horizontal redrills added. The MORES simulation specified total liquid production only. The quality of the history match was defined on predicted water production performance on a well-by-well basis.

To input the data set into 3DSL the major assumption required was that the system be strictly incompressible. The assumption was not a major limitation in this case since the voidage replacement ratio was approximately 1 . No tuning of the streamline history match was performed. Figure 2.10 summarizes the field oil and water production rates for MORES and 3DSL compared with the field data. Post-breakthrough prediction is excellent, although there is some deviation in the 3DSL results after 1985. We believe that this is a result of over prediction of water rates at two southern producers and is due to assuming only a single rock type within 3DSL.

For this 21,000 gridblock model, there was only a minimal speed advantage using 3DSL compared with the fully implicit MORES model. This is a result of historical well data limiting the time step size to 6 month intervals. However, we were also able to run a downscaled 201,000 gridblock model with 3DSL in 10.5 CPU hours. The ability to run a fine scale model translates into either incorporating more heterogeneity in the existing model, or including the entire field. A field-wide simulation eliminates the need to account for leak-off in pattern type simulations and gives true field-wide performance predictions. 


\subsection{Discussion and Limitations}

The streamline method is ideally suited for large, heterogeneous, multiwell problems that are convectively dominated. This is a class of problems that is particularly challenging for conventional numerical techniques. For these cases, the streamline simulator can represent the difference between solving a problem and not solving it at all. However, the method is not applicable to all situations and the results presented here do ignore the following effects:

1. We have assumed that the fluids are incompressible. For compressible flow, streamlines are still defined, but they do not always originate or end at wells. The method can accommodate compressibility by solving a mass balance equation along the streamlines. However, for highly compressible single-phase flow, such as primary production, frequent recomputation of the pressure field is necessary and the method is unlikely to offer a significant speed advantage over conventional techniques.

2. The current method does not allow for transport between streamlines due to capillary effects or transverse dispersion. These effects could be accommodated using operator-splitting [58]. Fluids would be moved diffusively along the underlying grid after the convective and gravity steps. For situations where these effects are dominant, again the streamline method may offer little speed advantage over existing methods.

Because of the decoupled nature of the streamline method, it is easily extended to more general displacements by simply solving a different mass conservation equation along each streamline. For example, we extended the method to compositional displacements in Chapter 3 [204], where multi-component phase behavior effects are accounted for in the $1 \mathrm{D}$ solver. We observe that the advantages of reduced numerical diffusion and increased speed for FCM and waterflood displacements are amplified for compositional displacements.

\subsection{Conclusions}

1. The underlying idea of the streamline method is to decouple a 3D problem into multiple 1D problems. Fluid transport is separated from the underlying grid and instead occurs along a dynamically changing streamline grid. The main advantage is that the grid CFL conditions are eliminated from fluid transport giving global time step sizes that are independent of the underlying grid constraints. Additionally the method reduces numerical diffusion. A streamline-based grid is ideal for convective dominated displacements in heterogeneous media.

2. The ability to take large time steps and a reduced number of pressure solves gives speedup factors between 1 and 2 orders of magnitude over conventional finite-difference methods. For waterflood and FCM displacements considered here, only 10's of pressure solves over 2 PVI were required, whereas conventional IMPES methods required 1000's or pressure solves and convective steps due to the grid CFL constraints. As shown, this increased efficiency translates into easily being able to solve million gridblock models.

3. The streamline method exhibits convergence as the time step size is reduced. The maximum time step size is dependent on the nonlinearity of the problem - the greater the nonlinearity, the smaller the time step size. In terms of front speeds, for all but cases with significant gravity effects, fronts can move at substantially greater than the minimum of 1 gridblock per time step. In comparison, conventional IMPES methods gave average front speeds substantially less than 1 gridblock per time step.

4. The streamline method was tested on a 56 well field waterflood with 30 years of production history. History matching results compared very well with a conventional simulation, although no tuning of the streamline model was performed. 


\subsection{Nomenclature}

$C=$ concentration

$D=$ depth from datum, $\mathrm{L}$

$E_{R}=$ dimensionless recovery efficiency

$f_{j}=$ fractional flow of phase $j$

$\vec{G}_{j}=j$ phase velocity due to gravity, L/t

$g=$ gravitational acceleration constant, $\mathrm{L} / \mathrm{t}^{2}$

$g_{v, x}=$ velocity gradient across gridblock in $x$-direction, $1 / \mathrm{t}$

$h=$ height of model, $\mathrm{L}$

$\overrightarrow{\vec{k}} \quad=$ absolute permeability tensor, $\mathrm{L}^{2}$

$k_{r j}=$ relative permeability of phase $j$

$\bar{k}_{H}=$ average horizontal permeability, $\mathrm{L}^{2}$

$\bar{k}_{V}=$ average vertical permeability, $\mathrm{L}^{2}$

$L=$ distance between producer and injector, $\mathrm{L}$

$m_{c}=$ center of mass

$N_{g} \quad=$ gravity number

$n_{p}=$ number of phases

$p=$ Pressure, $\mathrm{M} / \mathrm{t}^{2} \mathrm{~L}$

$S_{j}=$ saturation of phase $j$

$S_{j}^{c}=$ saturation of phase $j$ after convective step

$s=$ spatial distance coordinate along a streamline, $\mathrm{L}$

$t=$ time, $\mathrm{t}$

$t_{D}=$ dimensionless time

$u_{t}=$ total Darcy velocity, $\mathrm{L} / \mathrm{t}$

$v=$ interstitial velocity, $\mathrm{L} / \mathrm{t}$

$x_{e}=x$-position of streamline exit location, $\mathrm{L}$

$x_{i}=x$-position of streamline inlet location, $\mathrm{L}$

$x, y, z=$ spatial coordinates

$\overline{\Delta p}_{H}=$ average pressure drop in horizontal direction, $\mathrm{M} / \mathrm{t}^{2} \mathrm{~L}$

$\Delta t=$ global time step size, $\mathrm{t}$

$\Delta t_{1 D}=$ time step size within 1D solver, $\mathrm{t}$

$\Delta t_{e, x}=$ time-of-flight required to reach an $x$-exit face, $\mathrm{t}$

$\Delta \rho=$ fluid density difference, $\mathrm{M} / \mathrm{L}^{3}$

$\zeta=$ local streamline coordinate, $\mathrm{L}$

$\lambda_{c}=$ permeability correlation length

$\lambda_{g}=$ total gravity mobility, $1 / \mathrm{Lt}$

$\lambda_{t}=$ total mobility, Lt $/ \mathrm{M}$

$\mu_{j}=$ viscosity of phase $j, \mathrm{M} / \mathrm{Lt}$

$\rho_{j}=$ density of phase $j, \mathrm{M} / \mathrm{L}^{3}$

$\tau=$ time of flight, $\mathrm{t}$

$\phi \quad=$ porosity

$\omega=$ Todd \& Longstaff mixing parameter 


\subsection{Appendix: Tracing Streamlines}

Streamlines are launched uniformly across the face of a gridblock containing an injector, with the number of streamlines launched proportional to the flux across the face. Thus each streamline between all producers and injectors carries the same flux. Not every gridblock in the domain will contain a streamline based on launching a finite number of streamlines. For these missed gridblocks we define a streamline by simply tracing back from each missed gridblock until an injector is reached.

Tracing streamlines from injectors to producers is based on the analytical description of a streamline path within a gridblock as outlined by Pollock [180] and Datta-Gupta \& King [38]. The underlying assumption is that the velocity field in each coordinate direction varies linearly and is independent of the velocities in the other directions. This method is attractive because it is analytical and consistent with the governing material balance equation.

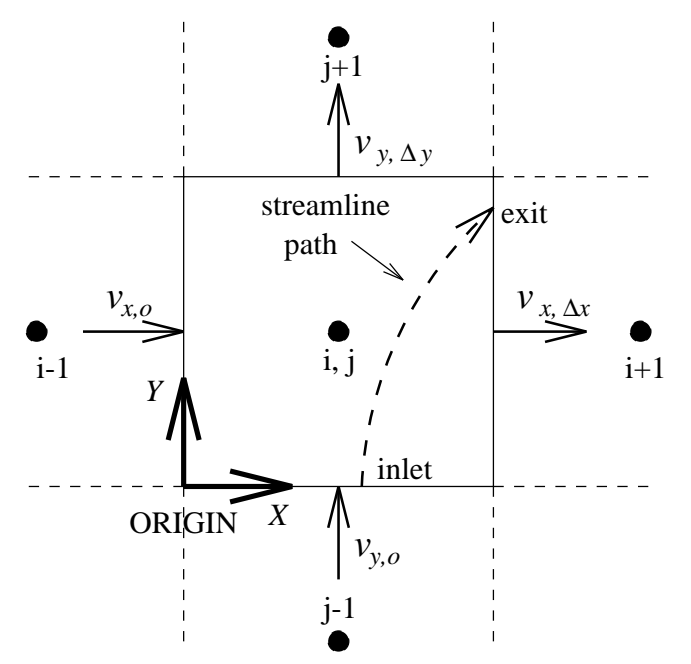

Figure 2.11: Schematic of a streamline path through a 2D gridblock of dimensions $\Delta x$ by $\Delta y$.

Consider the two-dimensional gridblock in Figure 2.11, for which we know the interstitial velocity field and have defined a local coordinate system and origin. The velocity in the $x$-direction, $v_{x}$, is defined as

$$
v_{x}=v_{x, o}+g_{v, x}\left(x-x_{o}\right),
$$

where $g_{v, x}$ is the velocity gradient across the gridblock and is given by

$$
g_{v, x}=\left(v_{x, \Delta x}-v_{x, o}\right) / \Delta x
$$

Knowing that $v_{x}=d x / d t$, one can integrate Eq. 2.13 to yield the time required to reach an $x$-exit face $\Delta t_{e, x}$ as

$$
\Delta t_{e, x}=\frac{1}{g_{v, x}} \ln \left\{\frac{v_{x, o}+g_{v, x}\left(x_{e}-x_{o}\right)}{v_{x, o}+g_{v, x}\left(x_{i}-x_{o}\right)}\right\},
$$

where $x_{i}$ is the inlet position and $x_{e}$ is the exit position, in the $x$-coordinate direction. Similarly, the times required to reach the exit faces in the $y$ or $z$ directions are given by

$$
\Delta t_{e, y}=\frac{1}{g_{v, y}} \ln \left\{\frac{v_{y, o}+g_{v, y}\left(y_{e}-y_{o}\right)}{v_{y, o}+g_{v, y}\left(y_{i}-y_{o}\right)}\right\},
$$


and

$$
\Delta t_{e, z}=\frac{1}{g_{v, z}} \ln \left\{\frac{v_{z, o}+g_{v, z}\left(z_{e}-z_{o}\right)}{v_{z, o}+g_{v, z}\left(z_{i}-z_{o}\right)}\right\} .
$$

The correct face which the streamline will exit is that which requires the smallest value of $\Delta t_{e}$ calculated from Eqs. 2.15, 2.16, and 2.17. Knowing the minimum time the exact exit location of the streamline is determined by rewriting Eqs. 2.15, 2.16, and 2.17 as

$$
\begin{aligned}
& x_{e}=\frac{1}{g_{v, x}}\left(v_{x, i} \exp \left\{g_{v, x} \Delta t_{e}\right\}-v_{x, o}\right)+x_{o}, \\
& y_{e}=\frac{1}{g_{v, y}}\left(v_{y, i} \exp \left\{g_{v, y} \Delta t_{e}\right\}-v_{y, o}\right)+y_{o}, \\
& z_{e}=\frac{1}{g_{v, z}}\left(v_{z, i} \exp \left\{g_{v, z} \Delta t_{e}\right\}-v_{z, o}\right)+z_{o},
\end{aligned}
$$

where $v_{i}$ represents the inlet-velocity and $v_{o}$ is the velocity at the origin coordinates.

For the case when velocity is uniform across a gridblock in a given direction $\left(g_{v}=0\right)$, Eq. 2.15, for example, simply becomes $\Delta t_{e, x}=\left(x_{e}-x_{i}\right) / v_{x, o}$ and Eq. 2.18 becomes $x_{e}=x_{o}+\Delta t_{e, x} v_{x, o}$. Finally, for the situation where a flow divide exists in say the $x$ direction within a gridblock, one must assure that the sign $v_{x}$ at the inlet location is the same as the sign of $v_{x}$ at a potential $x$ exit face. This check also avoids the possibility of calculating negative logarithms in Eqs. 2.15, 2.16, and 2.17. 


\title{
3. A Streamline-Based 3D Field-Scale Compositional Reservoir Simulator
}

\author{
Marco R. Thiele, Rod P. Batycky, and Martin J. Blunt
}

This chapter presents the extension of the streamline approach to full-field, three-dimensional (3D) compositional simulation. The streamline technique decomposes a heterogeneous 3D domain into a number of one-dimensional (1D) streamlines along which all fluid flow calculations are done. Streamlines represent a natural, dynamically changing grid for modeling fluid flow. We use a 1D compositional finite-difference simulator to move components numerically along streamlines, and then map the 1D solutions back onto an underlying Cartesian grid to obtain a full 3D compositional solution at a new time level.

Because of the natural decomposition of the 3D domain into a number of $1 \mathrm{D}$ problems, the streamline approach offers substantial computational efficiency and minimizes numerical diffusion compared to traditional finite-difference methods. We compare our three and four component solutions with solutions from two finite difference codes, UTCOMP and Eclipse 300 (E300). These examples show that our streamline solutions are in agreement with the finite-difference solutions, are able to minimize the impact of numerical diffusion, are faster by orders of magnitude. Numerical diffusion in finite-difference formulations can interact with reservoir heterogeneity to substantially mitigate mobility differences and lead to optimistic recovery predictions.

We demonstrate the efficiency and usefulness of the streamline-based simulator on a 518,400 gridblock, 3D, heterogeneous, 36-well problem for a condensing-vaporizing gas drive with four components. We can simulate this problem on an average-size workstation in three CPU days. It takes approximately the same amount of time to simulate the upscaled 28,800 gridblock version of the problem using finite-differences.

We conclude with a qualitative discussion explaining the near-linear scaling of the streamline approach with the number of gridblocks and the cubic and higher scaling exhibited by one of the finite-difference codes.

\subsection{Introduction}

The use of streamlines and streamtubes to model convective displacements in heterogeneous media has been presented repeatedly since the early work by Muskat[146, 144, 145], Fay and Prats[61], and Higgins and Leighton[90, 91, 92]. Important subsequent contributions are due to Parsons[169], Martin and Wegner[132], Bommer and Schechter[20], Lake et al.[123], Mathews et al.[134], Emanuel et al.[52, 54, 53], Renard[184], and Hewett and Behrens[86].

Recently, streamline methods have received renewed attention by several groups as a viable alternative to traditional finite-difference (FD) methods for large, heterogeneous, multiwell, multiphase simulations, which are particularly difficult for FD simulators to model adequately[21, 22, $172,173,206,207,205,18,11,10,12,190]$. Large speed-up factors compared to traditional FD solutions, minimization of numerical diffusion and grid orientation effects, and the inherent simplicity of the approach offer unique opportunities for integration with modern reservoir characterization methods. Examples include ranking of equiprobable earth models, estimation of the uncertainty in production forecasts due to the uncertainty in the geological description, rapid assessment of

\footnotetext{
${ }^{2}$ The material in this chapter was presented at the 1997 SPE Annual Technical Conference and Exhibition in San Antonio, Texas, Oct 5-8, 1997, and is published as SPE paper 38889 in the conference proceedings.
} 
production strategies such as infill drilling patterns and miscible gas injection[54, 172, 10, 208, 51]. In addition, streamlines may offer an attractive alternative to well-known problems with upscaling of absolute and pseudorelative permeabilities by allowing larger geological models and requiring upscaling across a smaller range of scales[33, 9].

As discussed in Chapter 2, our streamline approach for reservoir simulation hinges on two important extensions to past streamline/streamtube methods: (1) the use of true 3D streamlines[180] and (2) and numerical solutions of the transport equations along periodically changing streamlines[20, 184]. With these extensions we have been able to simulate realistic fluid flow in detailed, heterogeneous, 3D reservoir models much more efficiently than FD methods[205, 10, 12].

We emphasize that reservoir simulation using streamlines is not a minor modification of current FD approaches, but instead represents a significant shift in methodology. By transporting fluids along periodically changing streamlines, the streamline approach is equivalent to a dynamically adapting grid that is decoupled from the underlying, static, grid used to describe the reservoir geology. The 1D nature of a streamline is translated into a 1D transport problem that can be solved easily and efficiently. This is considerably different from FD methods that use the same grid to solve both for pressure and for saturation/composition, and are forced to move fluids only along grid directions.

The implementation of our approach uses five key ideas:

Heterogeneity and Well Locations: Streamlines represent the natural grid to capture high and low flow regions due to well placements and reservoir heterogeneity[146, 169, 132, 52, 86]. Muskat first used streamline to estimate reservoir drainage volumes resulting from well placements. The use of streamlines to capture the impact of heterogeneity is more recent and is due to the work by Lake et al[123] and by Emanuel and co-workers[52, 54, 53, 134, 86].

3D Streamlines: Tracing 3D streamlines using a time-of-flight (TOF) approach as presented by Pollock[180] and Datta-Gupta and King[38]. Using a TOF formulation along streamlines is significantly easier than using a volumetric formulation along streamtubes, particularly for true 3D flow. It is straightforward to show that a volumetric coordinate along a streamtube is equivalent to a TOF coordinate along a streamline[205].

1D Numerical Solutions: Moving solutions forward in time numerically along 1D streamlines as proposed by Bommer and Schechter[20]. By using a numerical FD solution along streamlines extends the approach to general initial conditions, changing boundary conditions, and any type of displacement or recovery mechanism which can be formulated in one dimension.

Updating Streamlines: Capturing the changing total velocity field due to problem nonlinearities as well as time-varying boundary conditions (wells coming online as well as shutting-in) by periodically recalculating the streamlines[133, 184, 206, 207, 205, 11, 10, 12]. Because streamlines represent the natural flow grid along which fluids want to move, far fewer streamline updates (global pressure solves) are required to move the fluids forward in time compared to FD methods. This results in a significant computational efficiency. Combining numerical $1 \mathrm{D}$ solutions (point 3 above) with periodically updated 3D streamlines is the centerpiece of our approach. It allows to capture nonlinear flow mechanism in 3D while retaining the speed advantages that made approximate $2 \mathrm{D}$ streamtube methods attractive in the past.

Operator-Splitting: Using operator-splitting to capture flow mechanisms that are not aligned with the total velocity field, such as gravity[74, 22]. The inability of $2 \mathrm{D}$ streamtube methods in the past to account for gravity effects has been a long-standing criticism. By using operator splitting and 3D streamlines it is possible to account for any mechanism that is not aligned with the total velocity field, such as transverse diffusion, capillary crossflow, and gravity. 
Operator splitting is a well established mathematical concept and has been used in the past to solve convective-diffusive transport problems[58], transport problems on multiple grids[182], and transport problems with gravity $[82,22]$.

\subsection{Compositional Displacements}

In this chapter we consider the extension of the streamline approach to compositional displacements. We have described the application of the method to immiscible and miscible displacements in our previous work (see Chapter 2) [206, 208, 205, 18, 11, 10], and the reader is referred to those publications for additional details.

Modeling mass transfer effects correctly becomes imperative when designing injection processes that will enhance the recovery of oil compared to traditional waterflooding methods. For example, under the right physical conditions gas or solvent injection can lead to very efficient recovery processes, as capillary forces that cause entrapment in immiscible displacements are reduced leading to much lower residual oil saturations. If the injected solvent is completely miscible, i.e. only a single phase is formed over the range of pressures and temperatures present in the reservoir, then the displacement is said to be first-contact-miscible (FCM) and the residual oil saturation in a swept zone is theoretically zero. In many cases however, miscibility is achieved in-situ after the solvent has contacted the oil repeatedly and mass transfer occurs between the injected solvent and the oil in place. These cases are referred to as multicontact miscibility (MCM). If miscibility is never achieved for the range of pressures and compositions in a particular displacement, then the displacement is referred to as being immiscible, although mass transfer still occurs between the flowing phases. For a general overview on the subject the reader is referred to Stalkup's monograph[197].

Although the residual oil saturations that can be achieved by gas injection processes can be very low, there is an offsetting effect caused by the injected fluids usually being significantly more mobile and of lower density than the resident oil. Higher mobility can lead to channeling and viscous fingering[203]. A significant density contrast can lead to gravity override. These effects have been observed experimentally at the core scale by several investigators[27, 26, 191, 62] and have also been confirmed numerically. Experimental work has also underscored the importance of crossflow mechanism due to capillary forces and concentration gradients, but is remains unclear to what extent these mechanisms act at the scale of typical field-scale reservoir simulation gridblock and how they affect the flow between gridblocks at that scale.

It is generally agreed though, that successful modeling of gas injection processes at the field scale must be able to capture the relevant interactions of the first-order transport mechanisms with the geological description of the reservoir. The successful study of these interactions has proven to be difficult in the specific case of compositional displacements. Problems usually encountered in traditional immiscible displacements such as numerical diffusion and upscaling of absolute and relative permeabilities[33, 9] are exasperated in compositional displacements because of the strong nonlinearities introduced by the thermodynamic equilibrium calculations[34, 8]. The impact of numerical diffusion is to mitigate the mobility and density contrasts of the fluids through mixing and underestimate bypassing and gravity override[199, 110]. Upscaling of fine-scale heterogeneities tends to modify local contact efficiencies and the multicontact miscibility process[149, 8, 56]. An additional difficulty — which is unique to compositional modeling - is the need to reduce the large number of hydrocarbon components present in a real system to a number that can still represent the phase behavior of the system and is numerically feasible[150]. Unfortunately, attempts to use finer grids with more geological detail and/or more pseudocomponents for a better representation of the phase behavior quickly lead to computational costs which are beyond current simulator capabilities, particularly for full-field development studies. As a result, most field-scale compositional simulation use grids with order $10^{3}$ to $10^{4}$ gridblocks. 
Streamlines can offer an attractive alternative to FD methods because of the natural decomposition of a 3D, heterogeneous problem into a series of simpler 1D, homogeneous problems. The advantage comes from the fact that the $1 \mathrm{D}$ solutions along the streamlines are completely independent from the size of the original 3D problems as well as the magnitude of the absolute permeability. The computational requirements for large field-scale problems are thereby reduced significantly, and problems with numerical diffusion, upscaling, and equilibrium calculations are minimized leading to more accurate predictions.

\subsection{The Streamline Formulation}

Here we develop the streamline formulation under the special assumption that gravity, capillary, and diffusive forces are negligible. All of these effects can be included using operator splitting in the same way gravity is included in oil-water displacements[10, 22].

The multidimensional mass conservation equations for a chemical species, $i$, flowing under convective forces only can be written as

$$
\phi \frac{\partial C_{i}}{\partial t}+\vec{u}_{t} \cdot \nabla F_{i}=0 \quad, \quad i=1, \ldots, n_{c}
$$

where

$$
C_{i}=\sum_{j=1}^{n_{p}} x_{i j} \rho_{j} S_{j} \quad, \quad j=1, \ldots, n_{p}
$$

are the local moles of component $i$ and

$$
F_{i}=u_{D} \sum_{j=1}^{n_{p}} x_{i j} \rho_{j} f_{j} \quad, \quad j=1, \ldots, n_{p}
$$

is the molar flux of component $i . n_{p}$ is the number of phases, $n_{c}$ is the number of components, $x_{i j}$ is the mole fraction of component $i$ in phase $j, \rho_{j}$ is the density of phase $j, S_{j}$ is the saturation of phase $j, u_{D}$ is the total dimensionless volumetric velocity, $\phi$ is the porosity, and $f_{j}$ is the fractional flow of phase $j$ given by

$$
f_{j}=\frac{k_{r j} / \mu_{j}}{\sum_{k=1}^{n_{p}} k_{r k} / \mu_{k}} .
$$

Here $k_{r j}$ is the relative permeability of phase $j$ and $\mu_{j}$ is the viscosity of phase $j$.

The streamline approach centers on transforming Eq. 3.1 into a 1D problem along streamlines. To do that we determine the time-of-flight[180, 38] (TOF) along a streamline, which is given by

$$
\tau=\int_{0}^{\zeta} \frac{\phi}{u_{t}} d s
$$

and leads to the definition

$$
\left|u_{t}\right| \frac{\partial}{\partial s} \equiv \vec{u}_{t} \cdot \nabla=\phi \frac{\partial}{\partial \tau}
$$

Using Eq. 3.6 it is possible to rewrite Eq. 3.1 as a one-dimensional formulation for each component

$$
\frac{\partial C_{i}}{\partial t}+\frac{\partial F_{i}}{\partial \tau}=0 \quad, \quad i=1, \ldots, n_{c} .
$$

The total velocity field, $u_{t}$, necessary to determine the TOF using Eq. 3.5 can be determined by solving the total mass balance equation

$$
\sum_{i=1}^{n_{c}}\left\{\phi \frac{\partial C_{i}}{\partial t}+\vec{u}_{t} \cdot \nabla F_{i}=0\right\}
$$


for pressure and then using Darcy's law to determine the local velocity field. For the work presented here, we have approximated Eq. 3.8 by

$$
\nabla \cdot \overrightarrow{\vec{K}} \cdot\left(\lambda_{t} \nabla P\right)=0
$$

where $\overrightarrow{\vec{K}}$ is the local permeability tensor and $\lambda_{t}$ is the total mobility given by

$$
\lambda_{t}=\sum_{j=1}^{n_{p}} k_{r j} / \mu_{j}
$$

Eq. 3.9 assumes that the pressure distribution solved under the assumption of incompressibility is close the solution obtained assuming a compressible system. For systems with a small pressure drop compared to the average reservoir pressure between injectors and producers — which we consider here - this is probably not a bad assumption. We are currently pursuing the extension to a fully compressible system, in which Eq. 3.8 is used to find the total velocity field rather than Eq. 3.9. This involves only a minor modification in the tracing algorithm and is a straightforward extension.

Although Eq. 3.9 does not account for compressibility and volume-change-on-mixing, these effects are accounted for in the 1D solution, Eq. 3.7, by solving for the dimensionless total velocity, $u_{D}$, which is no longer constant. Details of this are outlined in in the following section.

\subsection{One-Dimensional Compositional Solver}

Rewriting Eq. 3.1 as Eq. 3.7 decouples the global 3D, heterogeneous flow problem into a series of $1 \mathrm{D}$, homogeneous problems along streamlines. The original permeability distribution is now reflected by the geometry and the TOF distribution of a streamline. Using Eq. 3.7 allows to simulate any type of transport mechanism by simply using the appropriate 1D solver along streamlines. For this work we have written a 1D compositional solver for Eq. 3.7 which is described next.

We solve Eq. 3.7 for the total moles of component $i$ using an explicit first-order in time, second-order in space TVD scheme[17]. The scheme can be improved to second-order time accuracy using a simple modification[185]. Let

$$
F_{i}=u_{D} \hat{F}_{i}
$$

then we can write the finite-difference form of Eq. 3.7 as

$$
C_{i, k}^{n+1}=C_{i, k}^{n}+\frac{\Delta t}{\Delta \tau}\left(\left[u_{D} \hat{F}_{i}\right]_{k-\frac{1}{2}}^{n}-\left[u_{D} \hat{F}_{i}\right]_{k+\frac{1}{2}}^{n}\right)
$$

where the intercell fluxes, $\hat{F}_{i}$, are approximated by

$$
\hat{F}_{i, k+\frac{1}{2}}^{n}=\hat{F}_{i, k}^{n}+\frac{\Phi_{i, k+\frac{1}{2}}^{n}}{2}\left(\hat{F}_{i, k+1}^{n}-\hat{F}_{i, k}^{n}\right)\left(1-\frac{\Delta t}{\Delta \tau}\right) .
$$

$\Phi$ is the van Leer limiter[212]

$$
\Phi=\frac{r+|r|}{1+|r|}
$$

and $r$ is a function of the ratio of adjacent flux differences

$$
r_{i, k+\frac{1}{2}}^{n}=\frac{F_{i}^{n}-F_{i-1}^{n}}{F_{i+1}^{n}-F_{i}^{n}} .
$$



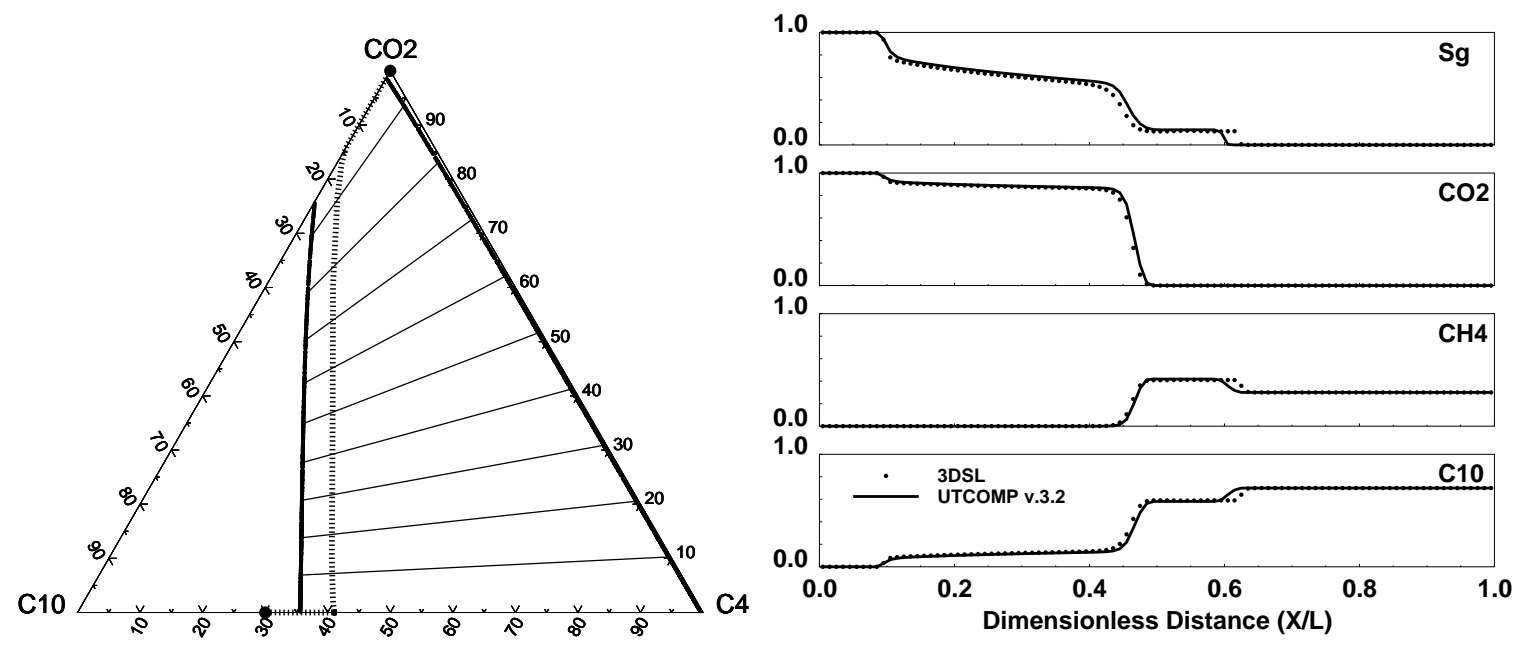

Figure 3.1: One-dimensional, TVD solutions at $t_{D}=0.5$ with 100 grid blocks using UTCOMP (v.3.2 $\mathrm{w} /$ automatic time stepping) and the $1 \mathrm{D}$ compositional simulator $(\mathrm{CFL}=0.5)$ used in this work for pure $\mathrm{CO}_{2}$ injection into a 30/70 mixture of $\mathrm{CH}_{4}$ and $\mathrm{C}_{10}$ at 1600 psia and $160^{\circ} \mathrm{F}$.

Here $k$ is the node counter of the discretized streamline, and $n$ is the local time level along each streamline. We then find the total mole fractions for each component using

$$
z_{l}=\frac{C_{l}}{\sum_{i=1}^{n_{c}} C_{i}} .
$$

Compressibility effects due to volume-change-on-mixing and pressure gradients are accounted for by solving iteratively for the total dimensionless velocity, $u_{D}$, together with Eq. 3.12 using a total mass balance equation of the form[44]

$$
u_{D, k+\frac{1}{2}}^{n}=\frac{\sum_{i=1}^{n_{c}}\left[\frac{\Delta \tau}{\Delta t}\left(C_{i, k}^{n}-C_{i, k}^{n+1}\right)+\left(u_{D} \hat{F}_{i}\right)_{k-\frac{1}{2}}^{n}\right]}{\sum_{i=1}^{n_{c}} \hat{F}_{i, k+\frac{1}{2}}} .
$$

Phase compositions, densities, and saturations are determined from an equilibrium calculation using the Peng-Robinson equation of state[177], an accelerated successive substitution algorithm[136], and a negative flash algorithm[225]. Liquid densities are corrected using volume translation[176]. Phase viscosities are calculated using the Lohrenz-Bray-Clark correlation[128].

Fig. 3.1 shows a comparison of the one-dimensional solution obtained using 3DSL with a Courant-Friedrichs-Lewy (CFL) number of 0.5 with the solution obtained using UTCOMPciteutc93 for a 3 component displacement at 1600 psia and $160^{\circ} \mathrm{F}$. UTCOMP is an implicit-pressure, explicitcomposition, isothermal, three-dimensional compositional simulator developed at the University of Texas at Austin with a third-order TVD scheme to control numerical diffusion. Except for a slight difference in the fastest front speed, the agreement is good.

\subsection{Two-Dimensional Solutions}

In this section we consider a three component (3C) condensing gas drive and a four component $(4 \mathrm{C})$ condensing-vaporizing gas drive through various heterogeneous cross sections. The 


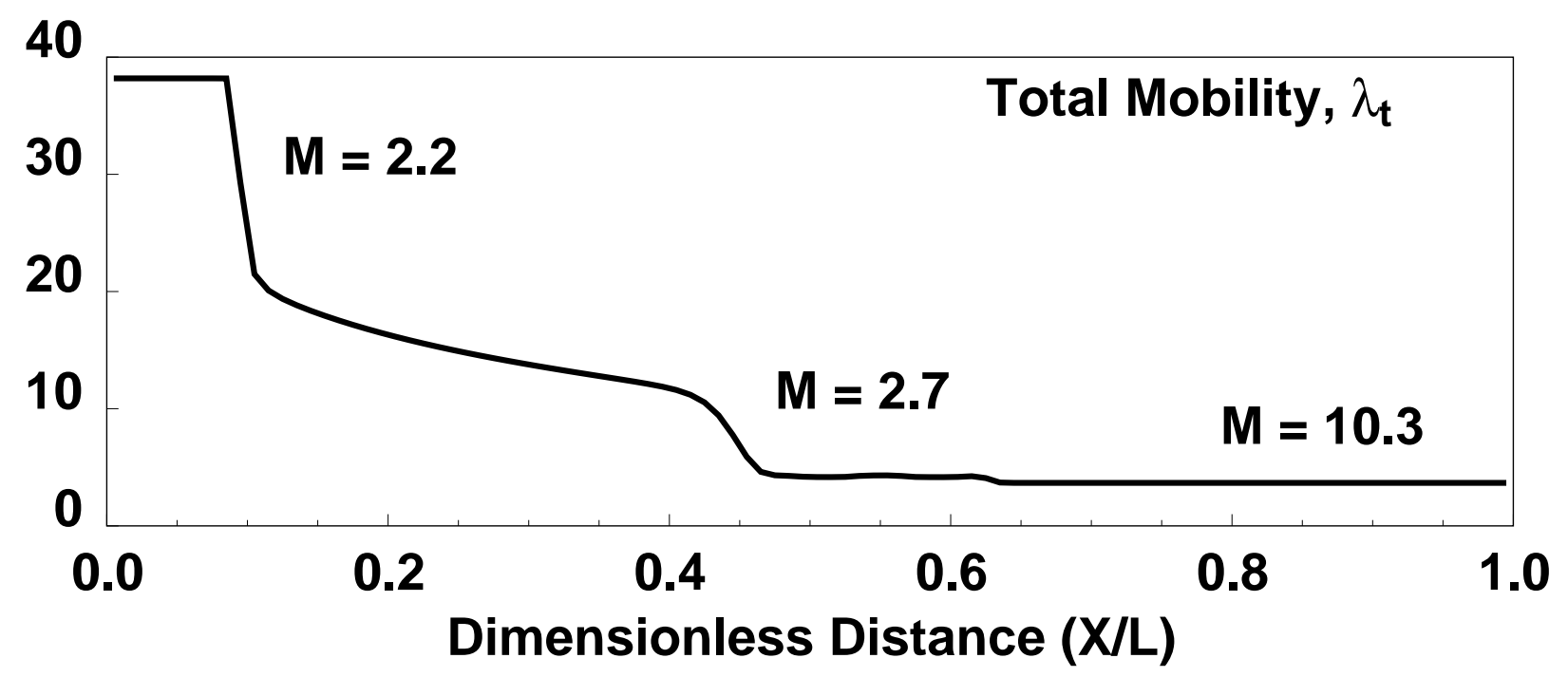

Figure 3.2: One-dimensional mobility ratio profile for the three component condensing problem of Fig. 3.1 at $t_{D}=0.5$.

aim of this section is two-fold: (1) to benchmark the solutions from the streamline approach with solutions obtained from UTCOMP and (2) to highlight the ability of the streamline approach to efficiently investigate the interaction of heterogeneity, numerical resolution (number of gridblocks), phase behavior and unstable flow. The second issue has been considered by many investigators[199, 29, 62, 8, 149, 166, 167]. The recurrent difficulty faced by these studies was the limited size of the numerical problem that could be considered because of the large computational resources generally required. Stalkup[199] attempted to overcome this limitation by extrapolating his results to an infinite number of gridblocks with some success. But if one-dimensional problems are any guide to the number of gridblocks required to obtain converged solutions, it is reasonable to assume that most $2 \mathrm{D}$ and $3 \mathrm{D}$ solutions presented in the literature are probably not converged solutions - particularly for heterogeneous domains - but instead represent an intermediate solution in a sequence of refined grids. This observation is demonstrated through an example in the next section.

\subsubsection{Three Component Condensing Gas Drive}

The injection of pure $\mathrm{CO}_{2}$ into a $3 \mathrm{O} / 70$ mole percent mixture of $\mathrm{CH}_{4}$ and $\mathrm{C}_{10}$ at 1600 psia and $160{ }^{\circ} \mathrm{F}$ is an example of a condensing gas drive. The corresponding 1D solution is shown in Fig. 3.1. For modeling $2 \mathrm{D}$ and 3D displacements, the expected nonlinearity (or instability) of the displacement can be estimated from the total mobility profile of the $1 \mathrm{D}$ solution. For the $3 \mathrm{C}$ case, Fig. 3.2 shows that the end-point mobility ratio of $M=10.3$ is divided into a leading $\mathrm{CO}_{2}$ front with a mobility ratio of $M=2.7$, a rarefaction wave, and a trailing front with a mobility ratio of $M=2.2$. Although the end-point mobility ratio is mitigated substantially by phase behavior effects alone, some nonlinearity can be expected due to the leading mobility ratio of $M=2.7$. In addition, because the leading $\mathrm{CO}_{2}$ shock is self-sharpening, numerical diffusion is expected to be less efficient in smearing out the mobility difference.

To investigate this displacement in 2D heterogeneous media, we considered an initial crosssection with $N_{x}=62$ and $N_{z}=25$ gridblocks (Fig. 3.3). Permeability varies over four orders 
of magnitude and has a correlation length of approximately 0.3 in the main direction of flow. A comparison of the spatial distribution of $\mathrm{CO}_{2}$ is shown in Fig. 3.3, and the corresponding recoveries are shown in Fig. 3.4. Both figures, Fig. 3.3 and Fig. 3.4, demonstrate that the streamline solution is in agreement with the solution given by UTCOMP. The UTCOMP solutions were found using the third-order TVD option for controlling numerical diffusion. For this problem, the streamline code (3DSL) required 0.75 hrs to generate the recovery curve up to 2 pore volumes (PV) injected, whereas UTCOMP required $18 \mathrm{hrs}$ - a speed-up of approximately 24 .

To further verify the $2 \mathrm{D}$ solution, we refined the $62 \times 25$ grid to $124 \times 50$ and to $248 \times 100$ by maintaining the same permeability distribution as the original $62 \times 25$ grid. Since the leading $\mathrm{CO}_{2}$ shock front has an unfavorable mobility ratio and is also self-sharpening, the increased numerical resolution of the local velocity field is expected to capture the instability of the problem. Fig. 3.5 shows the comparison between UTCOMP and 3DSL for the downscaled grids. The finer $248 \times$ 100 grid could not be run with UTCOMP due to prohibitively high computational costs. Three important comments can be made about the results shown in Fig. 3.5:

1. As the grid is refined, the streamline solution shows signs of instability at the leading $\mathrm{CO}_{2}$ front due to the unfavorable mobility ratio. This demonstrates that numerical diffusion in 3DSL is minimized to the point that we are able to see the onset of a viscous-fingering like behavior at the shock front and other small scale features of the displacement.

2. The UTCOMP solution for the $124 \times 50$ also has increased resolution compared to the $62 \times 25$ solution of Fig. 3.3. But unlike the streamline solution it appears as a sharpened image of the original $62 \times 25$ grid without noticeable changes in the small scale features of the $\mathrm{CO}_{2}$ front. This points to the problem that FD-models can require grid refinement by several powers of 2 before any noticeable change in the flow behavior can be observed. This despite higher order numerical schemes.

3. We could not simulate the $248 \times 100$ grid with UTCOMP because the computational expense was simply too high. We believe that this is not because of specific inefficiencies in UTCOMP, but rather because the underlying finite-difference scheme combined with the strong nonlinearities of the equilibrium calculations make refinement studies of the sort presented here very expensive for FD methods. In many cases studies of this sort are not possible using FD.

The speed-up of 3DSL compared to UTCOMP for the $124 \times 50$ grid was $92(92 \mathrm{hrs}$ for UTCOMP vs. $1 \mathrm{hr}$ for $3 \mathrm{DSL})$ to reach $2 \mathrm{PV}$ injected.

\subsubsection{Four Component Condensing-Vaporizing Gas Drive}

The displacement of a $3 \mathrm{C}$ mixture of $\mathrm{CH}_{4} / \mathrm{C}_{6} / \mathrm{C}_{16}(20 / 40 / 40)$ by an enriched gas mixture of $\mathrm{CH}_{4} / \mathrm{C}_{3}(65 / 35)$ at 2,000 psia and $200{ }^{\circ} \mathrm{F}$ exhibits a condensing behavior at the leading edge (the injected gas condenses into the resident oil) and a vaporizing behavior at the trailing edge (the injected gas volatilizes the resident oil)[109, 230]. Because the injected composition is close to the minimum enrichment composition for miscibility, the displacement can be characterized as being near-miscible[109]. Fig. 3.6 shows the 1D solution obtained using UTCOMP and 3DSL. The end-point mobility ratio for this $4 \mathrm{C}$ problem is $M \approx 8.4$. As a result of the near-miscible nature of the displacement, most of the mobility contrast $(M \approx 7.2)$ occurs over a small region (Fig. 3.6), and thus one may expect a $2 \mathrm{D}$ solution to be more unstable than the $3 \mathrm{C}$ problem considered in the previous section. But a finer 1D solution with 500 gridblocks (Fig. 3.6) reveals that the mobility contrast of $M \approx 7.2$ in fact occurs over two rarefaction waves and a self-sharpening shock[109], with the mobility ratio across the shock being $M \approx 2$. Thus, although the two-phase region is significantly smaller than in the $3 \mathrm{C}$ component case, the mobility contrast is mitigate significantly 
by the spreading nature of the two rarefaction waves. The instability for this displacement then is expected to be primarily due to the self-sharpening shock following the rarefaction waves.

We considered this problem in our previous work with analytical solutions along streamtubes. Because mapping analytical solutions along streamtubes overestimates the mobility contrast of a displacement, we indeed found solutions that were characterized by a very large amount of instability (long, wispy channels) as shown in Fig. 3.7. In contrast, the UTCOMP solution appeared to be characterized by a large amount of mixing resulting in a negligible mobility contrast[207].

We revisit the problem here using numerical solutions along streamlines. Fig. 3.7 shows the gas saturation maps at $t_{D}=0.4$ for the three displacements. The numerical solution obtained from 3DSL is clearly different from our first solution obtained using streamtubes in that it exhibits less channeling. But the 3DSL solution is also different from the UTCOMP solution, in that there appears to be more detail in the saturation distribution and signs of some channeling, particularly due to the self-sharpening shock following the rarefaction waves. The similarity between the 3DSL and UTCOMP solutions in Fig. 3.7 shows the ability of the streamline approach to capture the compositional nature of the problem correctly. It also suggests that for this displacement, the nearmiscible condition does not pose a significant problem in terms of the instability of the displacement. UTCOMP required 86 hrs to generate the saturation map shown in Fig. 3.7, whereas 3DSL required 0.6 hrs. This represents a speed-up factor of 143 .

\subsubsection{Sensitivity on Injected Composition.}

The speed and accuracy of the streamline approach clearly offers unique opportunities to revisit outstanding problems in predicting the effectiveness of enriched gas injection in heterogeneous media as a way to enhance production of hydrocarbons. As an example, we consider the sensitivity of the $4 \mathrm{C}$ condensing-vaporizing displacement on the level of enrichment of the injected mixture. This is a relevant questions since increasing enrichment will lower the residual oil saturation and increase the local contact efficiency, but at the same time may cause bypassing and early breakthrough due to the unstable nature of the displacement. Finding the optimal level of enrichment, that will maximize oil recovery for a given description of the phase behavior and geological setting entails many forward simulations with sufficient geological and numerical resolution. We emphasize that the solution to such an exercise will depend strongly on the physical scale of the problem and the resulting interaction of viscous, gravity, capillary, and diffusive forces at that scale[26, 27, 191]. Since we are neglecting gravity, capillary, and diffusive mechanisms here, the results are meant primarily to demonstrate the efficiency of the streamline approach, rather than to reveal any novel physical insight.

Fig. 3.8 shows the result of the sensitivity exercise through a $128 \times 64$ permeability field with 5 orders of magnitude difference in permeability. The three injection compositions mixtures of $\mathrm{CH}_{4} / \mathrm{C}_{3}-(85 / 15),(65 / 35)$, and $(45 / 55)$ mole percent - represent immiscible, MCM, and FCM displacements respectively of the initial oil composition. We also used a simple linear interpolation for the relative permeabilities to account for the fact that as the displacement goes from immiscibility to miscibility the relative permeabilities tend to straight lines and the residual saturations go to zero. For this particular example, Fig. 3.8 shows that injection of a $\mathrm{CH}_{4} / \mathrm{C}_{3}$ mixture that achieves first-contact miscibility with the resident oil will be the most efficient displacement. We believe that the primary reason for this is that in particular this case reservoir heterogeneity has a first order effect compared to displacement instabilities. It is the geology that is dictating the

primary channels of the solvent. The cumulative simulation time for the three cases on a standard workstation was $16 \mathrm{hrs}$. 


\subsection{Three Dimensional Solution}

In the previous sections we studied cross-sectional models. However, due to the decoupling of fluid transport from the underlying geological grid, the streamline method is ideally suited for large, 3D, multiwell models dominated by heterogeneity. Because of grid CFL constraints, these problems are particularly difficult for conventional FD methods.

As a result, the streamline approach offers unique opportunities to model field-scale problems with an acceptable level of geological resolution. Although upscaling will still be necessary to go from the fine-scale earth models of $\mathcal{O}\left(10^{7-9}\right)$ gridblocks to the reservoir simulation scale of $\mathcal{O}\left(10^{3-6}\right)$, the level of upscaling required will be significantly less for simulations using streamlines.

As an example, we simulated the $4 \mathrm{C}$ problem discussed in the previous section on a $180 \times 180 \times 16=518,400$ heterogeneous Cartesian grid with a total of 36 wells for a period of $0.7 \mathrm{PVs}$ injected. The average pressure gradient between injectors and producers was $\Delta P \approx 180$ psia. The simulation run required approximately 3 days on a standard workstation (DEC AlphaServer $21004 / 275$ with $256 \mathrm{MB}$ of RAM). Although there are only 30 gridblocks between wells for this half-million gridblock example, it is a significant improvement in resolution compared to the limited number of gridblocks usually used in FD field-scale models. Fig. 3.9 shows recovery and breakthrough curves for $\mathrm{C}_{3}$ obtained using UTCOMP and Eclipse 300[72] (E300). The upscaled $60 \times 60 \times 8$ model was generated from the fine-scale $180 \times 180 \times 16$ model using simple geometric averaging of the absolute permeabilities. More sophisticated upscaling techniques may have reduced the difference between fine-scale and coarse-scale results[33, 9]. Fig. 3.9 is a reminder that the difference in the solution due to upscaling of the geological model, pseudoization of the phase behavior, and the usually low numerical resolution between wells poses a challenging problem for traditional FD approaches for modeling field-scale compositional problems. Streamlines may offer a simple yet effective solution to increase the geological detail and resolution between wells using a fraction of the computational costs required by FD. As we show in the following section, the streamline approach may in fact allow to model problems that are beyond current hardware capabilities for FD methods. For this example, solution of the $60 \times 60 \times 8=28,800$ model with E300 required approximately the same CPU time as the $180 \times 180 \times 16=518,400$ model with 3DSL. We estimated the runtime for the $180 \times 180 \times 16$ model with Eclipse in the tens of years (Fig. 3.10).

\section{7 $\quad$ Timing and Scaling}

In our previous work[205, 10] we found speed-up factors between 10 and 500 for waterflooding and FCM displacements in 2D and 3D heterogeneous domains and under a variety of flow conditions, geological descriptions, and boundary conditions. In most cases, the speed-up resulted from the reduced number of global pressure solves and large convective steps allowed by 3DSL.

Fig. 3.10 shows scaling results we found for the 3D, 4C problem described previously. We generated Fig. 3.10 by starting with the fine-scale model of $180 \times 180 \times 16$ and then considered upscaled models $60 \times 60 \times 8,45 \times 45 \times 8$, and $36 \times 36 \times 8$. We could only run the later three models with E300 using our available computational resources. For comparison, we also show the scaling of 3DSL on the same 3D problem for a waterflood. Although the results in Fig. 3.10 are specific to the example we studied, they show that the streamlines solutions scale near-linearly with the number of gridblocks, $N_{g b}$, whereas the FD solution obtained with Eclipse 300 scales approximately as $N_{g b}^{3.4}$.

The scaling behavior of each method can be explained qualitatively by considering the 
expression for the total run time, $T$, given by

$$
T=N_{t s} \times \Delta t_{t s}
$$

where $N_{t s}$ is the number of timesteps and $\Delta t_{t s}$ is the time required per timestep. $\Delta t_{t s}$ is the sum of the time required to invert the pressure matrix, $\Delta t_{p}$, and the time to move the saturation/concentration forward in time, $\Delta t_{S}$. It is reasonable to assume that for each method, $\Delta t_{t s}$, is roughly the same and with a near linear scaling. For example, our public-domain multigrid[119] solver scales near-linearly, $\Delta t_{p} \propto N_{g b}^{1.3}$.

The efficiency of the streamline approach compared to FD is derived from the fact that the number of global timesteps, $N_{t s}$, is independent of the number of gridblocks of the system, because fluid transport is decoupled from the underlying grid. Instead, it is directly related to the nonlinearity of the problem (mobility contrast/gravity number) and the number of well-events (changing boundary conditions). A good example of this is tracer flow, where for constant boundary conditions, only a single global timestep is required, irrespective of the level of heterogeneity and the number of gridblocks. The reason that the magnitude of heterogeneity is irrelevant is because the $1 \mathrm{D}$ solution along the streamline is homogeneous, with all the heterogeneity buried in the TOF.

A finite-difference code, on the other hand, must take many timesteps that are directly dictated by the size of the gridblocks and the magnitude of the heterogeneity present. It is probably the changing level of heterogeneity (higher extremes of permeability with increasing grid resolution) that causes the cubic scaling exhibited by the FD code in Fig. 3.10. For homogeneous systems, the number of timesteps in FD are expected to scale linearly with $N_{g b}$. But as heterogeneity is added to the model, finer grids with higher permeability contrasts will cause the number of timesteps to scale with a power greater than one. Assuming a near-linear scaling for the pressure solution and the explicit timestepping, combined with a quadratic scaling of the number of timesteps would explain the $N_{g b}^{3.4}$ seen in Fig. 3.10 for the FD code.

\subsection{Conclusions}

We have extended the streamline approach to 2D and 3D compositional displacements by using a general 1D numerical solver along streamlines. The 1D solution accounts for compressibility and volume-change-on-mixing by solving for the total velocity. The advantages of using streamlines for two-phase immiscible as well as FCM displacements are amplified for compositional displacements:

1. By decoupling the 1D transport problem from the underlying geological grid, streamlines allow to retain the numerical accuracy required to model compositional displacements.

2. The streamline approach scales near-linearly with the number of gridblocks, which represents a considerable improvement over conventional FD methods.

3. The efficiency of the streamline approach offers a unique opportunity to revisit outstanding questions for designing field-scale enhanced-recovery processes.

4. Higher geological and numerical resolution reduces the number of scales that need to be crossed when going from the fine-scale earth model to the reservoir simulation model, thereby reducing the dependence on upscaling of absolute and relative phase permeabilities. This will lead to a significant improvement in the resolution of the transport problem and to more reliable predictions.

5. The increased numerical efficiency can be translated to a higher number of pseudocomponents for the description of the phase behavior, larger models, and better geological resolution. 


\subsection{Nomenclature}

$C_{i}=$ total moles of component $i$

$F_{i}=$ molar flux of component $i$

$f_{j}=$ fractional flow of phase $j$, dimensionless

$\overrightarrow{\vec{K}} \quad=$ absolute permeability tensor, $\mathrm{L}^{2}$

$k=$ node index for finite-difference scheme

$k_{r j}=$ relative permeability of phase $j$, dimensionless

$M=$ mobility ratio, dimensionless

$n_{c}=$ number of components

$n_{p}=$ number of phases

$N_{g b}=$ number of total gridblocks

$N_{t s}=$ number of global time steps

$n=$ time step level in finite-difference formulation

$P=$ Pressure $\mathrm{M} / \mathrm{T}^{2} \mathrm{~L}$

$P V=$ pore volumes, $L^{3}$

$r=$ ratio of successive flux differences

$S_{j}=$ saturation of phase $j$, dimensionless

$s=$ spatial distance coordinate along a streamline, $\mathrm{L}$

$t=$ time, $\mathrm{T}$

$t_{D}=$ dimensionless time

$\vec{u}_{t}=$ total Darcy velocity, $\mathrm{L} / \mathrm{T}$

$u_{D}=$ dimensionless total velocity

$x_{i, j}=$ mole fraction of component $i$ in phase $j$, dimensionless

$z_{i}=$ total mole fraction of component $i$, dimensionless

$\Delta t=$ time step size in 1D solution, $\mathrm{T}$

$\Delta t_{p}=$ CPU time required per global pressure solution, $\mathrm{T}$

$\Delta t_{S}=$ CPU time required per global convective step, $\mathrm{T}$

$\Delta t_{t s}=$ CPU time required per global time step, $\mathrm{T}$

$\Phi=$ van Leer limiter for TVD scheme

$\phi \quad=$ porosity, dimensionless

$\rho_{j}=$ density of phase $j, \mathrm{M} / \mathrm{L}^{3}$

$\zeta=$ local streamline coordinate, $\mathrm{L}$

$\lambda_{t}=$ total mobility, $\mathrm{L}^{3} \mathrm{~T} / \mathrm{M}$

$\tau=$ time of flight, $\mathrm{T}$

$\mu_{j}=$ viscosity of phase $j, \mathrm{M} / \mathrm{TL}$ 


\section{Permeability}
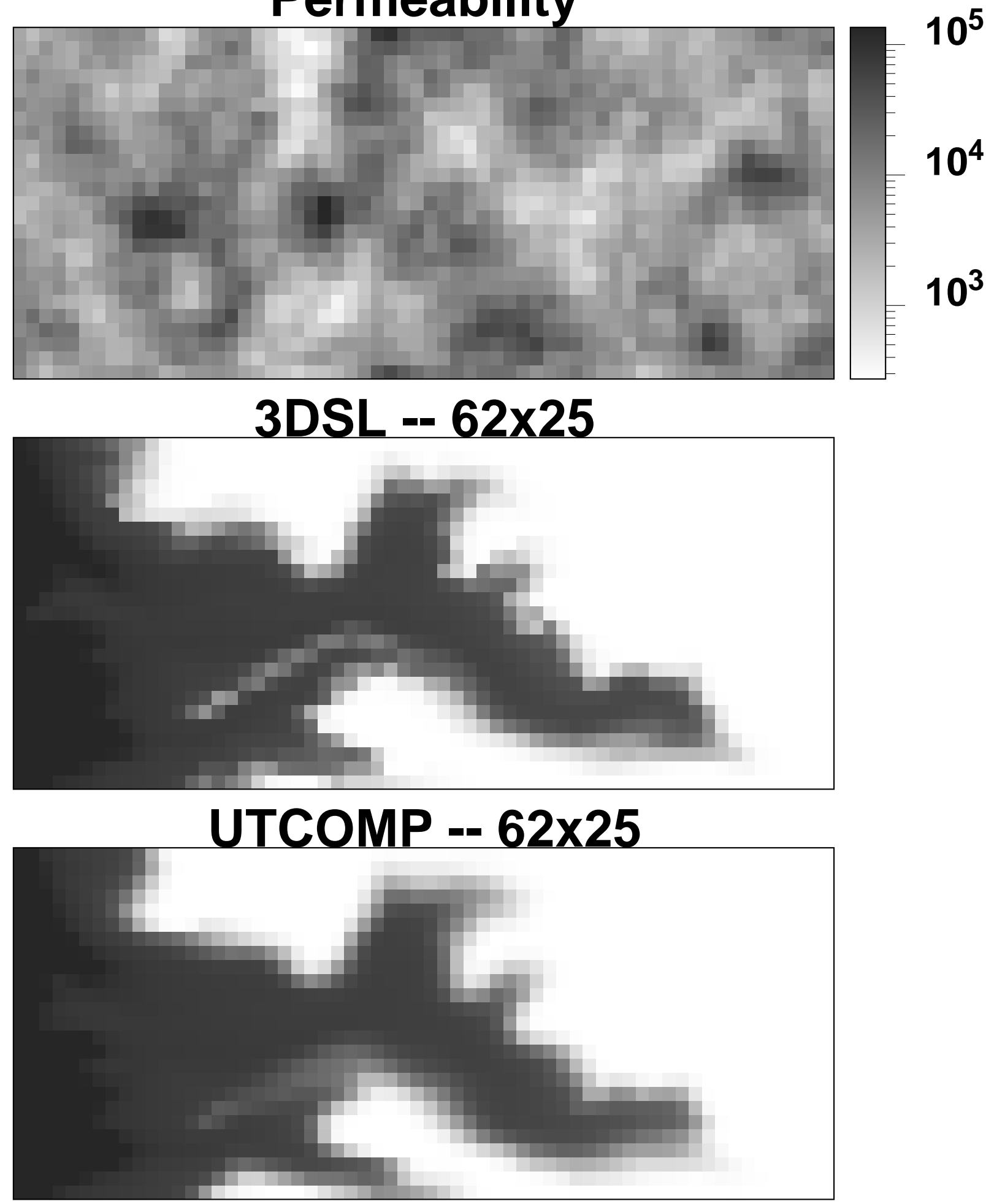

Figure 3.3: Permeability field $(62 \times 25)$ and spatial distribution of $\mathrm{CO}_{2}$ for $3 \mathrm{DSL}$ and UTCOMP at $t_{D}=0.5$. 


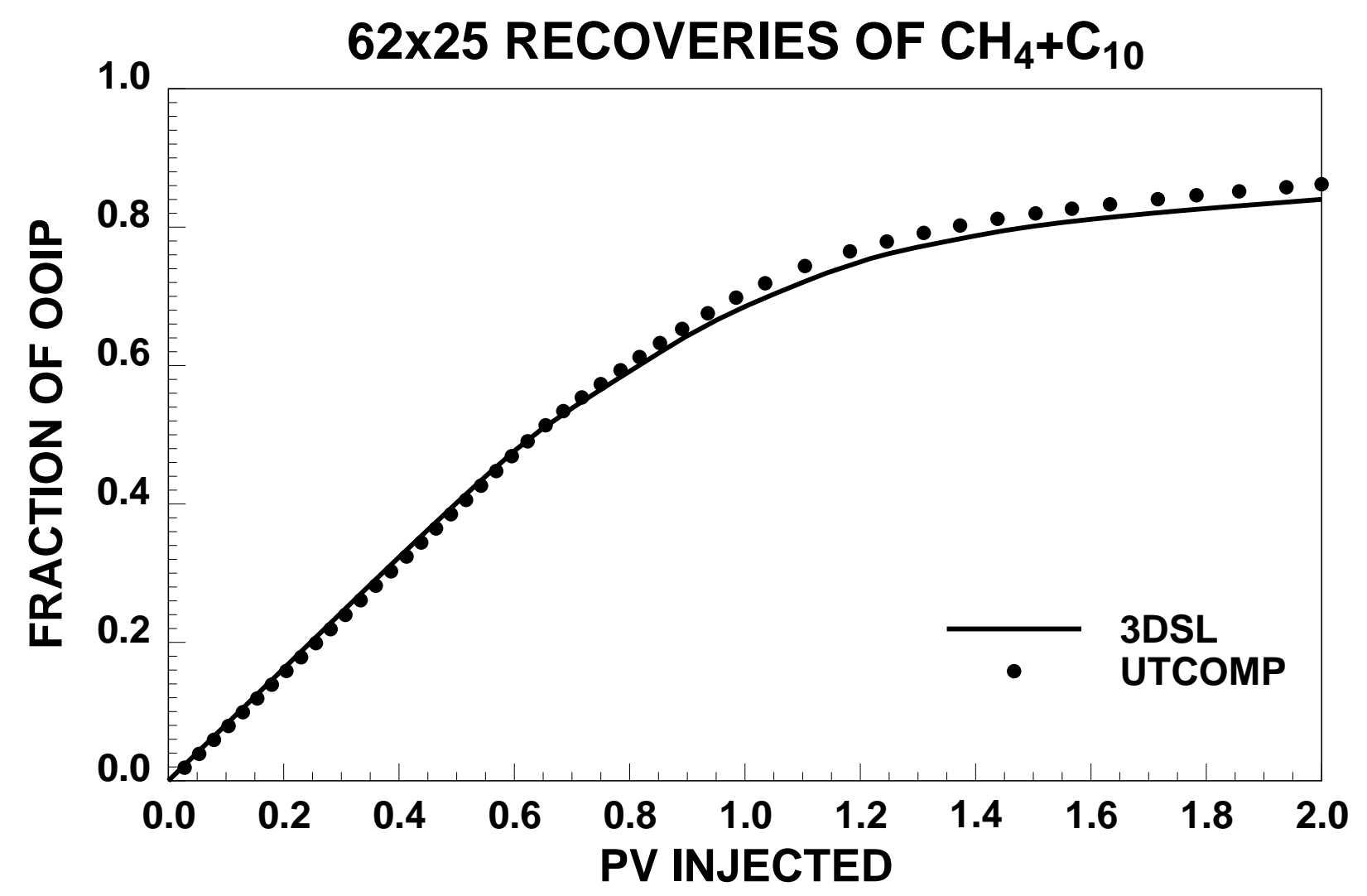

Figure 3.4: Cumulative recoveries of $\mathrm{CH}_{4}$ plus $\mathrm{C}_{10}$ for 3DSL and UTCOMP. 

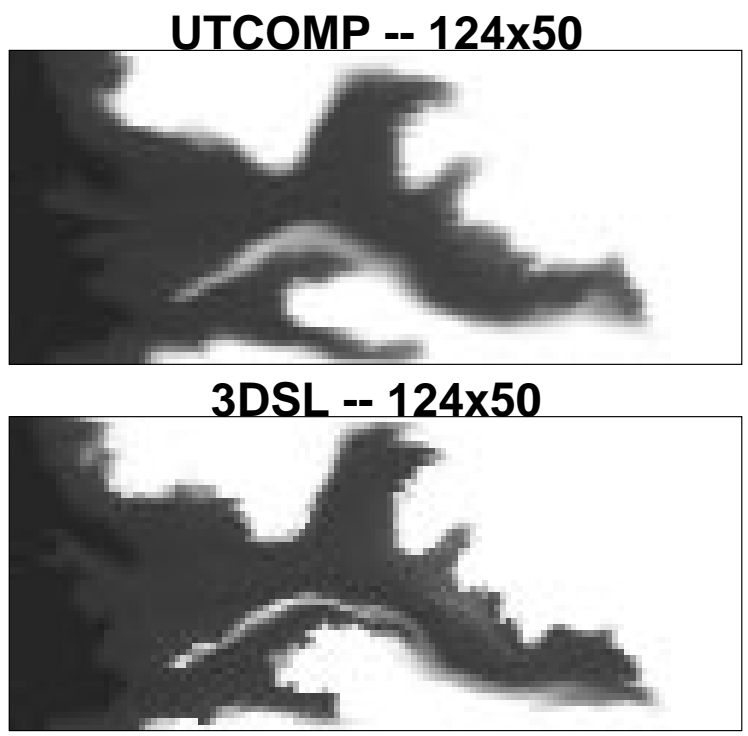

3DSL -- 248x100

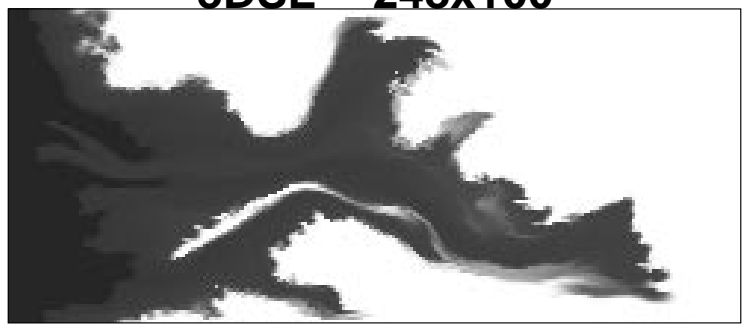

Figure 3.5: Spatial distribution of $\mathrm{CO}_{2}$ for $3 \mathrm{DSL}$ at $t_{D}=0.5$ for grids $124 \times 50$ and $248 \times 100$ compare to the UTCOMP solution for grid $124 \times 50$. 


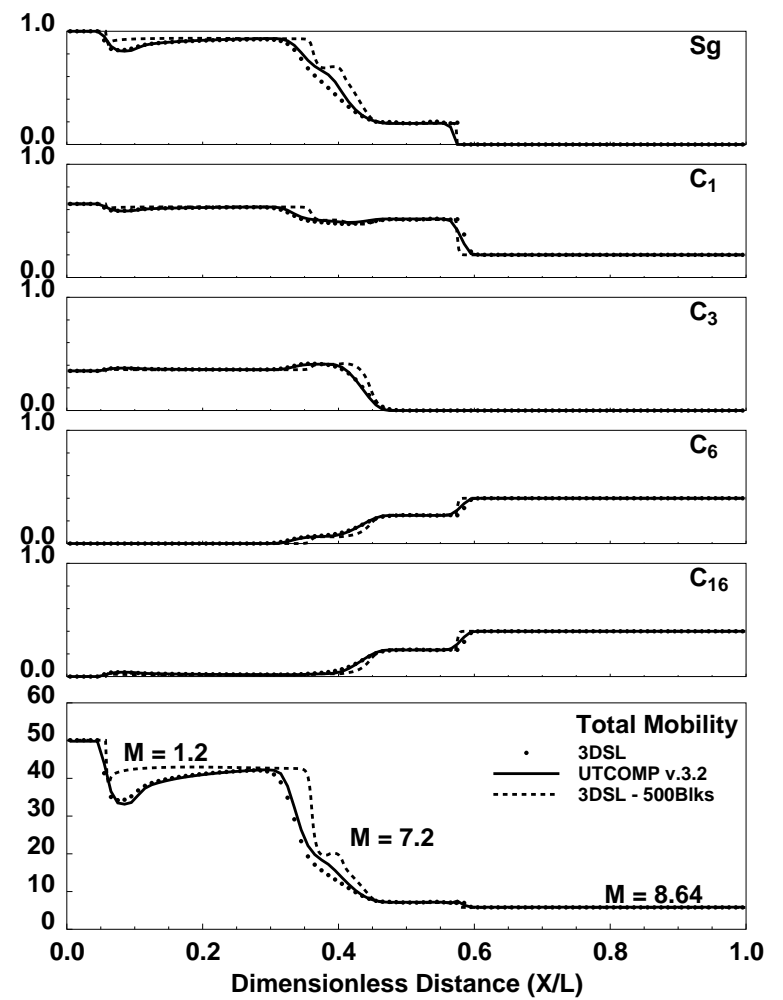

Figure 3.6: One-dimensional, TVD solution with 100 gridblocks using UTCOMP (v.3.2 w/ automatic time stepping) and TVD solutions with 100 and 500 gridblocks using $3 \mathrm{DSL}(\mathrm{CFL}=0.25)$ for $\mathrm{CH}_{4} / \mathrm{C}_{3}$ $(65 / 35)$ injection into a $20 / 40 / 40$ mixture of $\mathrm{CH}_{4} / \mathrm{C}_{6} / \mathrm{C}_{16}$ at 2000 psia and $160^{\circ} \mathrm{F}$. 


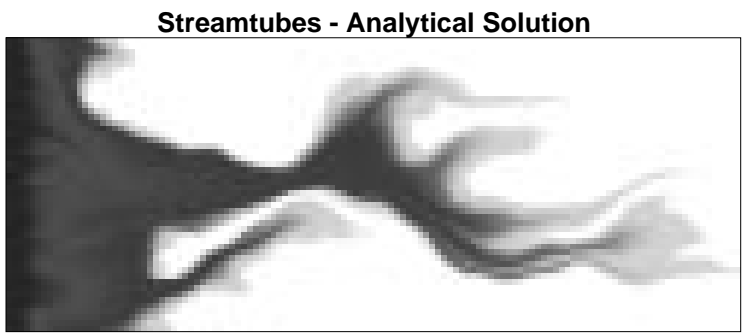

3DSL - Numerical Solution TVD (0.6 hr)

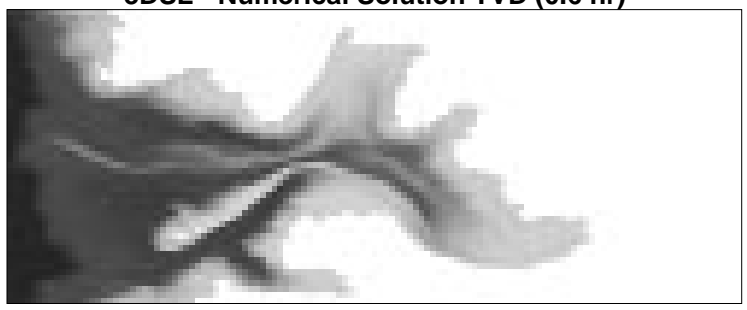

UTCOMP - TVD (86 hr)

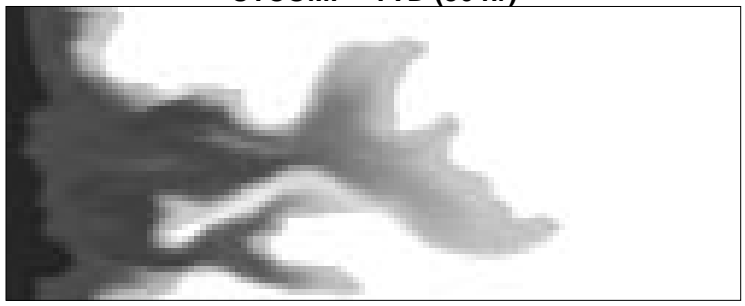

Figure 3.7: Saturations maps at $t_{D}=0.4$ for the displacement of a $20 / 40 / 40$ mixture of $\mathrm{CH}_{4} / \mathrm{C}_{6} / \mathrm{C}_{16}$ at 2000 psia and $160^{\circ} \mathrm{F}$ by a $65 / 35$ mixture of $\mathrm{CH}_{4} / \mathrm{C}_{3}$ through a heterogeneous cross-section with $125 \times 50$ gridblocks. 


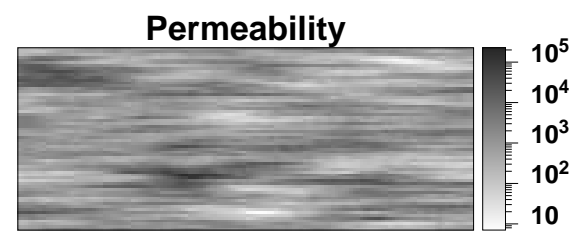

$\mathrm{C} 1 / \mathrm{C} 3=85 / 15$

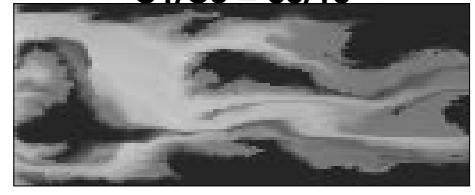

$\mathrm{C} 1 / \mathrm{C} 3=65 / 35$

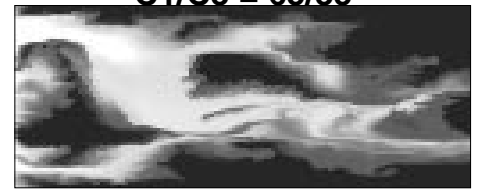

$\mathrm{C} 1 / \mathrm{C} 3=45 / 55$

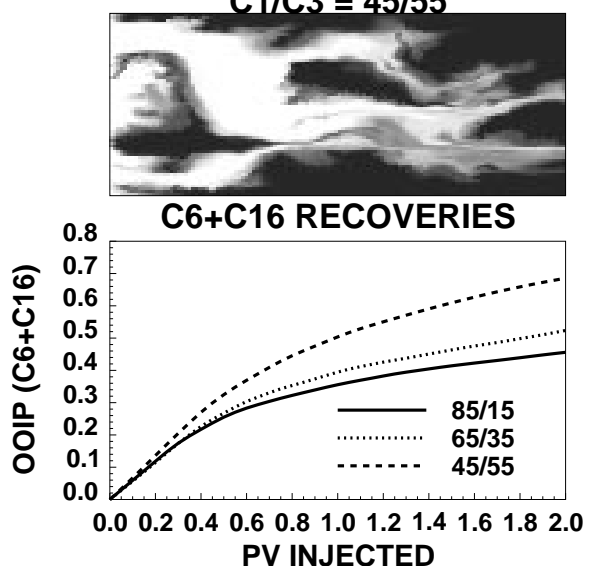

Figure 3.8: Sensitivity of the displacement of a 20/40/40 mixture of $\mathrm{CH}_{4} / \mathrm{C}_{6} / \mathrm{C}_{16}$ at 2000 psia and $160^{\circ} \mathrm{F}$ on the enrichment level of the injected mixture of $\mathrm{CH}_{4} / \mathrm{C}_{3}$. The permeability field has 5 orders of magnitude difference in permeability. The maps show spatial distribution of $\mathrm{C}_{3}$. Recoveries are for $\mathrm{C}_{6}$ plus $\mathrm{C}_{16}$. 

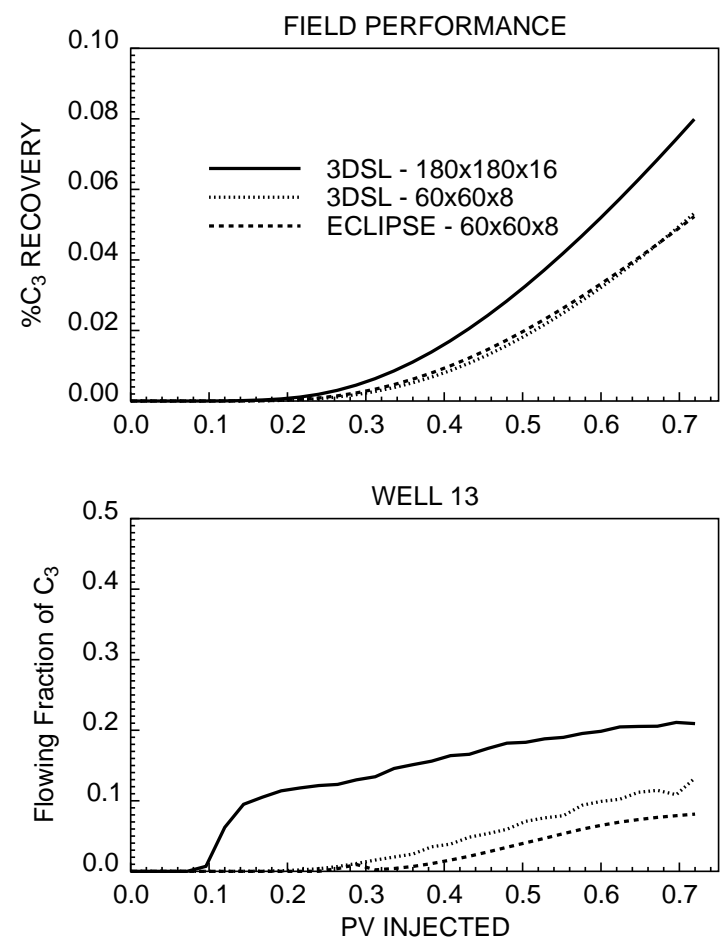

Figure 3.9: 3D solutions with $180 \times 180 \times 16=518,400$ gridblocks and with $60 \times 60 \times 8=28,800$ gridblocks for the $4 \mathrm{C}$ problem of Fig. 3.6. 


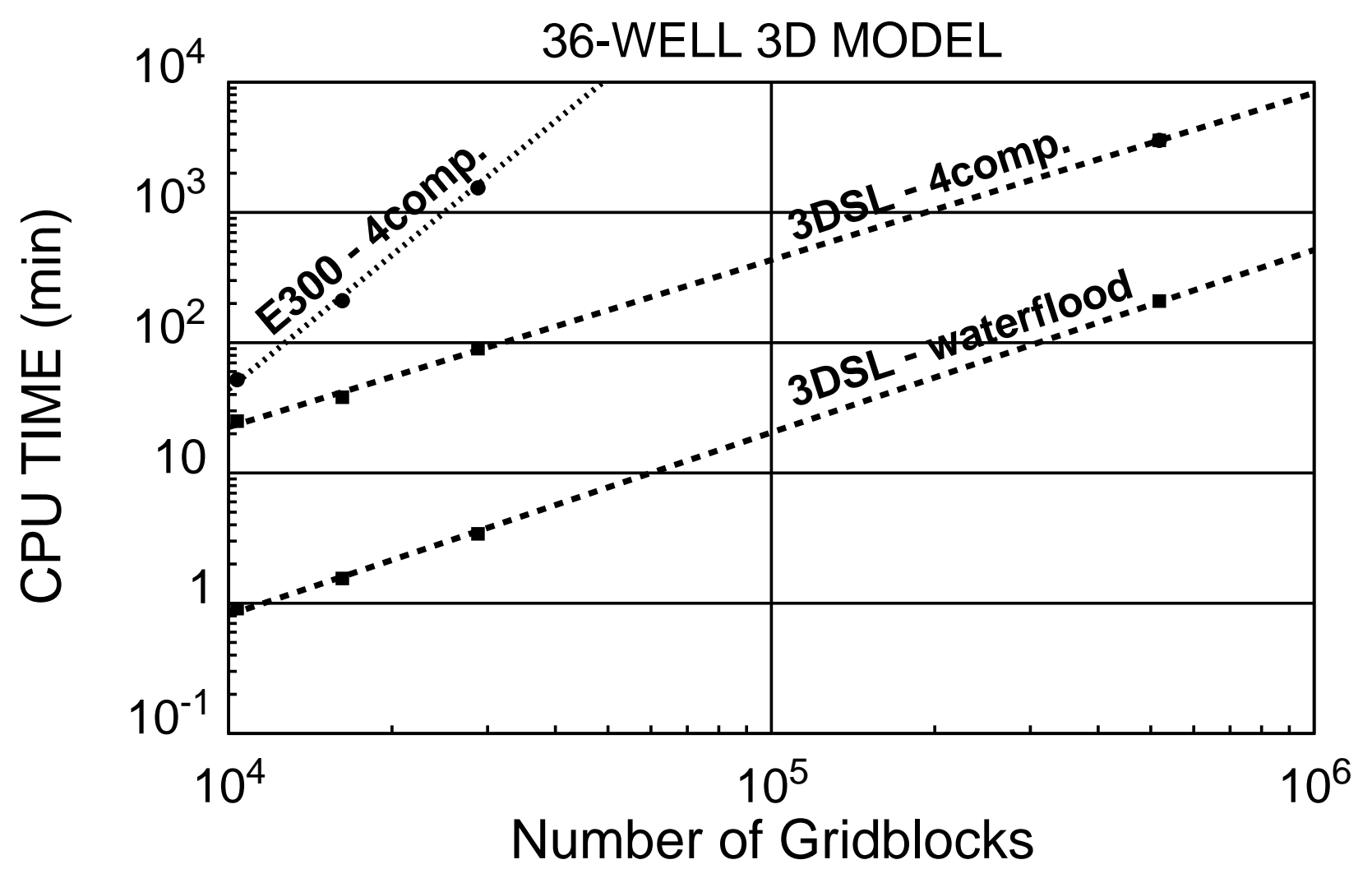

Figure 3.10: Scaling of compositional solution. 


\title{
4. Nested Gridding and Streamline Simulation
}

\author{
Yann Gautier, Martin J. Blunt, and Michael A. Christie
}

\begin{abstract}
Detailed reservoir models routinely contain $10^{6}$ to $10^{8}$ grid blocks. These models often cannot be used directly in a reservoir simulation because of the time and memory required for solving the pressure grid on the fine grid. In this chapter, we propose a nested gridding technique that efficiently obtains an approximate solution for the pressure field. The domain is divided into a series of coarse blocks, each containing several fine cells. Effective mobilities are computed for each coarse grid block and the pressure is then found on the coarse scale. The pressure field within each coarse block is computed using flux boundary conditions obtained from the coarse pressure solution. Streamline-based simulation is used to move saturations forward in time. We test the method for a series of example waterflood problems and demonstrate that the method can give accurate estimates of oil production for large $3 D$ models significantly faster than direct simulation using streamlines on the fine grid, making the method overall approximately up to 1,000 times faster than direct conventional simulation.
\end{abstract}

\subsection{Introduction}

Reservoir models containing millions of grid blocks frequently cannot be used directly in reservoir simulation since the time and memory cost is too high. Instead the reservoir simulation is performed on a coarser grid on which average multiphase flow properties are defined. The process of finding effective properties is called upscaling and in recent years many different methods have been proposed (see [31, 165] for recent reviews).

For single-phase flow problems, the absolute permeability has to be upscaled. Several reliable methods can be found in the literature [165]. In contrast, upscaling for multiphase flow is not so well established although several methods of "pseudoization" have been developed (see Barker [9] and references therein for a review on that subject). However most of the time the results are not robust and depend highly on the particular boundary conditions used.

Recently another approach has been considered: instead of doing the simulation only on a coarse gridding of the reservoir, both the fine and the coarse scales are considered. Gautier and Nœtinger [71] used this idea for single phase flow problems. They upscaled the full absolute permeability tensor using renormalization and they proposed an algorithm to construct the fine grid fluxes based on the the upscaled grid properties. The preferential flow paths were well detected but the orientation of the fluxes wasn't accurate due to a bias of the algorithm.

Ramé and Killough [182] used a multiscale method to solve miscible flow problems. They interpolated on a fine grid the pressure obtained from a coarser grid solution. They first used a collocation finite element method to interpolate, which leads to non physical oscillatory variations of the pressure. The results were then improved using a a spline method. The main problem of their method was that the mass conservation was not ensured from their interpolation scheme. Moreover the fine grid heterogeneities were not directly considered.

Verdière and Guérillot $[78,214]$ applied a dual scale approach for waterflooding problems that overcome the limitations of the method developed by Ramé and Killough [182]. As before, saturation was computed on a fine grid and the pressure on a coarser grid. They also associated two different time scales to the pressure and to the saturation equations. The algorithm they used

\footnotetext{
${ }^{3}$ The material in this chapter is published as "Nested Gridding and Streamline-Based Simulation for Fast Reservoir Performance Prediction," Computational Geosciences (1999) 3, 295-320.
} 
can be divided in different steps. First, considering the fine grid information, they upscaled values of the product of the absolute permeability and of the mobility (the transmissivity) using a simple algebraic rule. They then solved the pressure equation on the coarse grid. In the next step they computed the fine grid pressure and the fluxes using flux boundary conditions derived from the coarse grid pressure. With this information they updated the saturation solving the associated hyperbolic equation. All their computations were done using a finite difference simulator on a $2 D$ field with simple boundary conditions: prescribed rates or pressures on two opposite corners of the reservoir. They neglected gravity and capillary pressure. The results were on good agreement with the fine grid simulation leading to a precision gain over typical upscaling methods. Guedes and Schiozer [77] used a similar method with a different upscaling step. Instead of an algebraic rule, they used a pseudo method to obtain the upscaled transmissivities. They showed the improvment of their methods with respect to coarse grid simulations using upscaled absolute permeabilities or pseudo relative permeabilities.

In a subsequent paper, Verdière and Vignal [215] showed mathematically that their method converges to the exact solution using a finite volume formalism. They also computed numerical error estimates on a few test cases. In a more general framework, Hou and Wu [98] have developed a multiscale finite element approach for solving pressure-like equations. This is a mathematically rigorous technique whereas the method developed by Verdière and Guérillot is approximate and broadly corresponds to the first step of a multigrid method.

In this chapter, we will apply this type of algorithm to solve the pressure equation within a streamline-based multiphase flow simulation [10, 204]. Streamline-based simulation has been shown to give fast and reliable estimates of the solution of different flow simulation problems. The streamline method is briefly as follows. The grid pressure is first computed and the velocity field is derived using Darcy's law. Streamlines are then traced from injectors to producers. The next step is to solve the one-dimensional saturation equation on every streamline and map the saturations back to the original grid. The grid properties are finally updated and the pressure is solved for the next time step.

A multiscale approach for streamline-based simulation is appealing for several reasons. The first motivation is to increase the speed of the streamline method. Streamline methods can be up to 100 times faster and require less memory than a typical finite difference simulation [10, 204]. As we will see the new method will provide an additional speed-up factor of nearly one order of magnitude, with a saving of up to $40 \%$ of the memory cost. Hence, bigger problems can potentially be considered than by using conventional simulation. Another motivation is that using a multiscale method to solve the pressure equation can provide a fully parallel method since all the upscaling problems and the computation of the fine grid pressure are independent and can be made parallel easily. This is also the case for the saturation equation which has been split into series of small one-dimensional problems.

We will implement this nested gridding streamline-based flow simulation within a more general framework than the one used by Verdière and Guérillot $[78,214]$. We will show how to treat wells and how to incorporate $3 D$ effects by computing an equivalent gravity transmissivity. To upscale fine grid transmissivities, we will use the pressure solve technique which has been shown to be more robust than other upscaling methods [165, 171]. This step requires the solution of small linear systems to obtain the fine grid block pressure with prescribed boundary conditions. The exiting flux is then computed and the effective transmissivity is derived using Darcy's Law. To optimize the CPU cost of a pressure evaluation we will select different solvers for the different problems we encounter. For the small problems that we have to solve during the upscaling step and during the pressure computation on the fine grid we use a band solver that is particularly efficient for small linear systems. A multigrid or an iterative solver is used for larger problems on the coarse scale. Therefore nested gridding provides us a flexible way to save CPU time for the computation 
of the pressure since we adapt the type of solver used to the size of the problem considered.

First, we briefly review the streamline method, then we develop our new technique, and lastly, we present results in $2 D$ and $3 D$ with and without gravity.

\subsection{The Streamline Method}

We consider multiphase flow with the following assumptions: we neglect capillary, compressibility and dispersion effects. The streamline method is an IMPES method (IMplicit in Pressure and Explicit in Saturation). The pressure $p$ is solved using the following differential equation [5]:

$$
\nabla \cdot \mathbf{k}\left(\lambda_{t} \nabla p+\lambda_{g} \nabla D\right)=Q
$$

$D$ represents the depth, $Q$ the source terms due to wells, $\mathbf{k}$ the absolute permeability tensor, $\lambda_{t}$ and $\lambda_{g}$ the total mobility and total gravitational mobility respectively for $n_{p}$ phases, defined as:

$$
\lambda_{t}=\sum_{j=1}^{n_{p}} \frac{k_{r_{j}}}{\mu_{j}}, \quad \lambda_{g}=\sum_{j=1}^{n_{p}} \frac{k_{r_{j}} \rho_{j} g}{\mu_{j}}
$$

where $g$ is the gravitational acceleration constant and $\rho_{j}$ is the density of phase $j$. The material balance for phase $j$ leads to the saturation equation:

$$
\phi \frac{\partial S_{j}}{\partial t}+\mathbf{u}_{t} \cdot \nabla f_{j}+\nabla \cdot \mathbf{G}_{j}=Q_{w} f_{j, w}
$$

where $f_{j}$ represents the Buckley-Leverett fractional flow of phase $\mathrm{j}$ :

$$
f_{j}=\frac{k_{r_{j}} / \mu_{j}}{\sum_{m=1}^{n_{p}} k_{r_{m}} / \mu_{m}}
$$

and $\mathbf{G}_{j}$ is the velocity due to gravity effects:

$$
\mathbf{G}_{j}=g f_{j} \mathbf{k} \nabla D \sum_{m=1}^{n_{p}} \frac{k_{r_{m}}}{\mu_{m}}\left(\rho_{m}-\rho_{j}\right)
$$

The total velocity $\mathbf{u}_{t}$ is computed from the pressure solution of Eq. 4.1, using Darcy's law:

$$
\mathbf{u}_{t}=\mathbf{k}\left(\lambda_{t} \nabla p+\lambda_{g} \nabla D\right)
$$

In a conventional finite difference method, the saturation equation, Eq. 4.3 is solved directly on the $3 D$ field. With the streamline method, the problem is decoupled into a set of $1 D$ problems along streamlines.

The streamlines are traced from injectors to producers. Their paths are determined from the total velocity computed for each grid block face using an analytical method developed by Pollock [180]. The saturation is then moved along each streamline using a numerical solution of the $1 D$ problem. Finally, the saturation is mapped onto the grid blocks. The pressure field is then recomputed and new streamlines are traced. The different steps are pictured in Figure 4.1.

The streamline method has two main advantages over conventional finite difference simulators $[10,204]$. It requires fewer simulation time steps and it is less sensitive to numerical diffusion. The maximum time step between two pressure solves in a conventional IMPES simulator is conditioned by a global CFL constraint which requires that the fluid cannot move more than one grid block per time step. Typically, the CFL constraint is determined from the largest flow velocity in the whole field leading to unnecessarily small time steps. In the streamline method, a local 


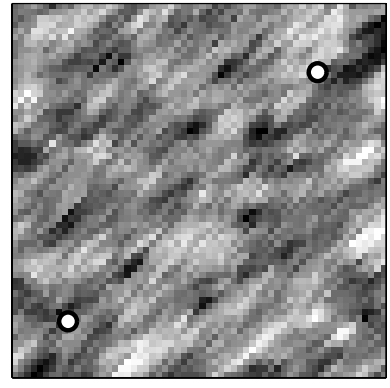

(a)

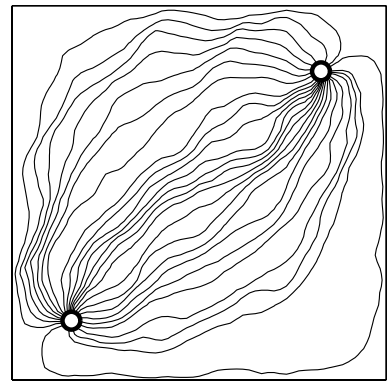

(c)

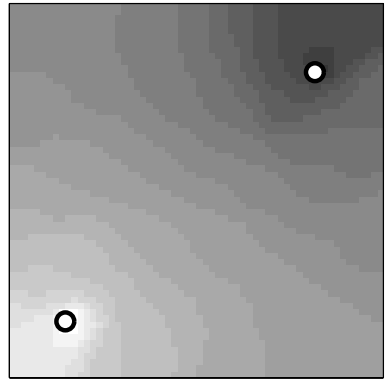

(b)

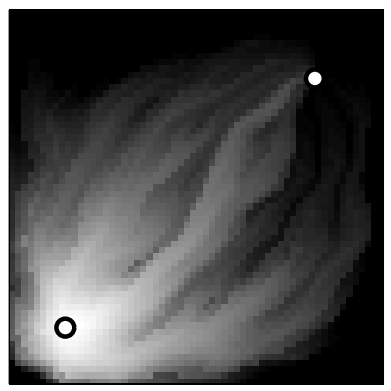

(d)

Figure 4.1: Description of the streamline method. Consider a permeability map (a) with injector and a producer wells. Solve the pressure field $(b)$. Compute the velocity field and trace streamlines (c). Move saturation along streamlines and compute the values of the saturation on the grid (d). 
CFL constraint is defined for every streamline and the saturation is updated using a $1 D$ solver. Large global time steps can be taken between pressure solves. The most time consuming step for both streamline and conventional approaches is the solution of the pressure equation Eq. 4.1. In our proposal method we use an approximate pressure solver based on nested gridding in order to decrease the CPU time of the simulation.

\subsection{Description of the New Method}

The basic idea of the nested gridding method is to decouple the pressure problem into a set of smaller problems on two scales: we consider a coarse grid superimposed on a fine grid (Figure 4.2). We assume that the initial petrophysical properties are defined on the fine grid. The first step of the nested gridding method is to upscale these properties on the coarse scale. The pressure equation Eq. 4.1 is solved on the coarse scale. The next step is to go back to the fine grid, solving a set of local problems within each coarse grid block using boundary conditions derived from the previous step.

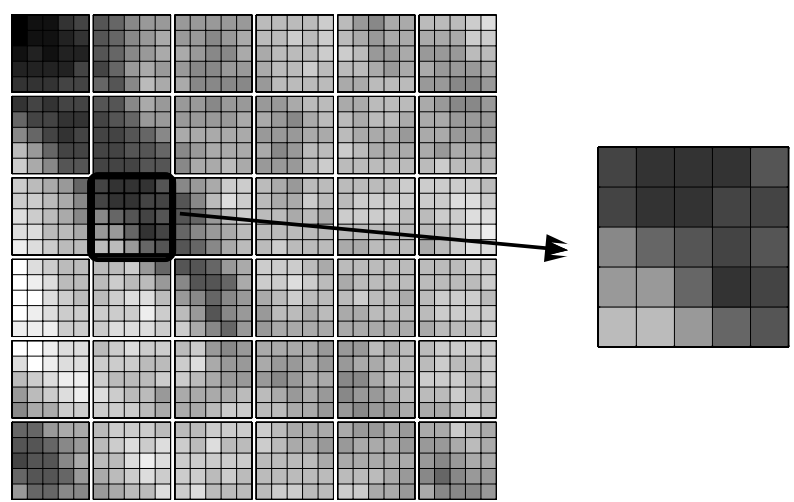

Figure 4.2: Representation of the coarse grid superimposed on a fine grid. The right figure represents a particular coarse grid block

Let us first recall the finite difference equation related to the pressure equation. Using a seven point stencil, the pressure is determined from the classical relation [5]:

$$
\begin{aligned}
& T_{z, k-\frac{1}{2}} P_{i, j, k-1}+T_{y, j-\frac{1}{2}} P_{i, j-1, k}+T_{x, i-\frac{1}{2}} P_{i-1, j, k} \\
- & P_{i, j, k}\left(T_{z, k-\frac{1}{2}}+T_{y, j-\frac{1}{2}}+T_{x, i-\frac{1}{2}}+T_{z, k+\frac{1}{2}}+T_{y, j+\frac{1}{2}}+T_{x, i+\frac{1}{2}}\right) \\
+ & T_{z, k+\frac{1}{2}} P_{i, j, k+1}+T_{y, j+\frac{1}{2}} P_{i, j+1, k}+T_{x, i+\frac{1}{2}} P_{i+1, j, k} \\
= & Q_{i, j, k}+G_{z, k-\frac{1}{2}} D_{i, j, k-1}-G_{z, k+\frac{1}{2}} D_{i, j, k}
\end{aligned}
$$

where the right hand side contains source terms induced by the boundary conditions $Q$ and by the difference of elevation between the considered grid block and its neighbors (for a cartesian grid only the neighbors in the vertical direction have a nonzero contribution). $i, j, k$ are respectively the indices of a block in the $x, y, z$ directions. $T_{\bullet, \bullet \pm \frac{1}{2}}$ represents the inter-block transmissivities which is the harmonic average of the block transmissivities. For example, in the $x$ direction, with $\left(\Delta x_{i}, \Delta y_{i}, \Delta z_{i}\right)$ representing the size of the grid block:

$$
T_{x, i+\frac{1}{2}}=\frac{2}{\frac{1}{T_{x, i}}+\frac{1}{T_{x, i+1}}}
$$


where the transmissivity of each fine grid block is:

$$
T_{x, i}=\frac{\Delta y_{i} \Delta z_{i}}{\Delta x_{i}} \lambda_{t, i} k_{x, i}
$$

$G_{\bullet, \bullet \pm \frac{1}{2}}$ represents the inter-block gravity transmissivities which is the harmonic average of the block gravity transmissivities. For example, in the $x$ direction,

$$
G_{x, i+\frac{1}{2}}=\frac{2}{\frac{1}{G_{x, i}}+\frac{1}{G_{x, i+1}}}
$$

where the transmissivity of each fine grid block is:

$$
G_{x, i}=\frac{\Delta y_{i} \Delta z_{i}}{\Delta x_{i}} \lambda_{g, i} k_{x, i}
$$

Eq. 4.7 can also be represented in a matrix form

$$
\mathbf{T P}+\mathbf{G D}=\mathbf{Q}
$$

where $\mathbf{T}$ and $\mathbf{G}$ are the transmissivity and gravity transmissivity matrices, $\mathbf{P}$ and $\mathbf{D}$ are vectors containing the pressure and the elevation of the different grid blocks.

\subsubsection{The Upscaling Step}

In the following we will focus on one particular type of upscaling: the pressure solve technique $[13,165,171,179,120]$. This method is widely used and it has been shown to be more robust than other faster techniques such as simple averaging rules and renormalization [165, 117, 71].

In our nested gridding method we compute two different upscaled quantities: the transmissivities and the gravity transmissivities. Let $N$ be the number of coarse grid blocks and $m$ the number of fine grid blocks contained in each coarse grid block. We take an isolated coarse grid block $(I, J, K)$ and solve the pressure equation (Eq. 4.7) on the underlying fine grid with suitable boundary conditions. To compute the effective transmissivities in the direction $d=x, y, z$, Eq. 4.7 is solved using constant pressure boundary conditions on the $d$ direction and no-flux boundary conditions on the other directions. We neglect gravity for these computations. Once the pressure is obtained inside the coarse grid block, the exiting flux $q^{c}$ is computed and the effective transmissivity of each coarse grid block is derived using Darcy's law. In the $x$ direction:

$$
T_{I, x}^{c}=\frac{q^{c}}{\Delta X_{I} \Delta P_{x}}
$$

where $\Delta P_{x}$ is the pressure drop and $\Delta X_{I}$ is the length of the coarse grid block in the $x$ direction. This process is repeated in the two remaining directions to obtain $\mathbf{T}^{c}=\left(T_{x}^{c}, T_{y}^{c}, T_{z}^{c}\right)$ for all the coarse grid blocks. The upscaling process in the $x$ direction is depicted in Figure 4.3.

To compute the effective gravity transmissivity, we are using the same method as before but with different boundary conditions. Since a Cartesian grid is considered, only the $z$ direction effective gravity transmissivity will have a nonzero value which we call $G_{I, z}^{c}$. To solve the fine scale problem, we consider no-flux boundary condition on the $x$ and $y$ faces of the block and a constant gravity $g$ along the $z$ direction. No pressure drop is applied on the two opposite faces in the $z$ direction. $G_{I, z}^{c}$ is computed from the exiting flux with:

$$
G_{I, z}^{c}=\frac{q^{c}}{g \Delta Z_{I}}
$$




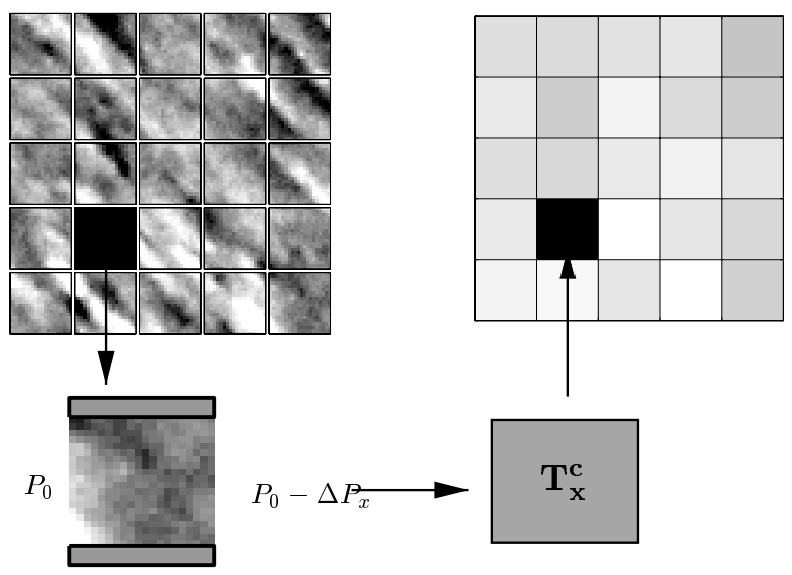

Figure 4.3: Upscaling of the transmissivities: the fine transmissivity map is divided into coarse grid blocks. The pressure equation is solved independently on each coarse grid block with a no flux boundary on all the faces except two opposing faces across which a prescribed pressure drop is applied. The upscaled values of the transmissivities are then obtained from the computation of the flux across the system.

The upscaling is repeated for every coarse grid block. Each coarse block is characterized by three upscaled transmissivities and one upscaled gravity transmissivity. Note that the effects of the fine grid saturation distribution is accounted for in $\mathbf{T}^{\mathbf{c}}$ and $G_{I, z}^{c}$, and that, in order to compute the different effective parameters we split the influence of the viscous and gravitational forces. The different upscaling steps are described in Figure 4.4.

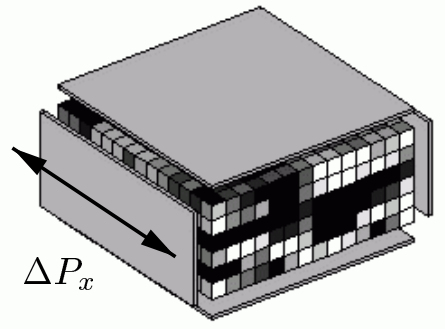

(a)

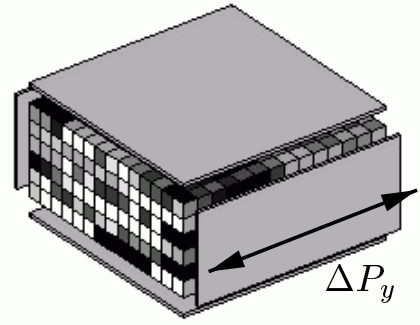

(b)

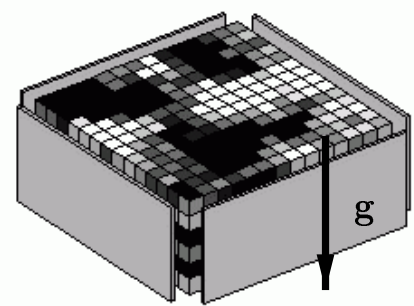

(d)

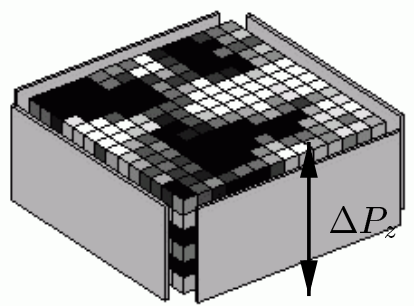

(c)

Figure 4.4: Upscaling of the transmissivity in the three directions $(a-c)$ where a constant pressure drop $\Delta P_{d}$ is applied on the direction $d$ and the gravity is set to $g=0$. (d) depicts the upscaling of the gravity transmissivity where $\Delta P_{z}=0$ and $g \neq 0$. 


\subsubsection{Pressure Solve on the Coarse Grid}

The second step of the method is to compute the pressure on the coarse scale using Eq. 4.7 with the effective transmissivities $\mathbf{T}^{c}$. Here the right hand side contains the source terms induced by the wells. To represent the wells we use the Peaceman model [170]. In the next step, the pressure at the coarse scale will be used to derive the pressure field at the fine scale.

\subsubsection{Computation of the Pressure on the Fine Grid.}

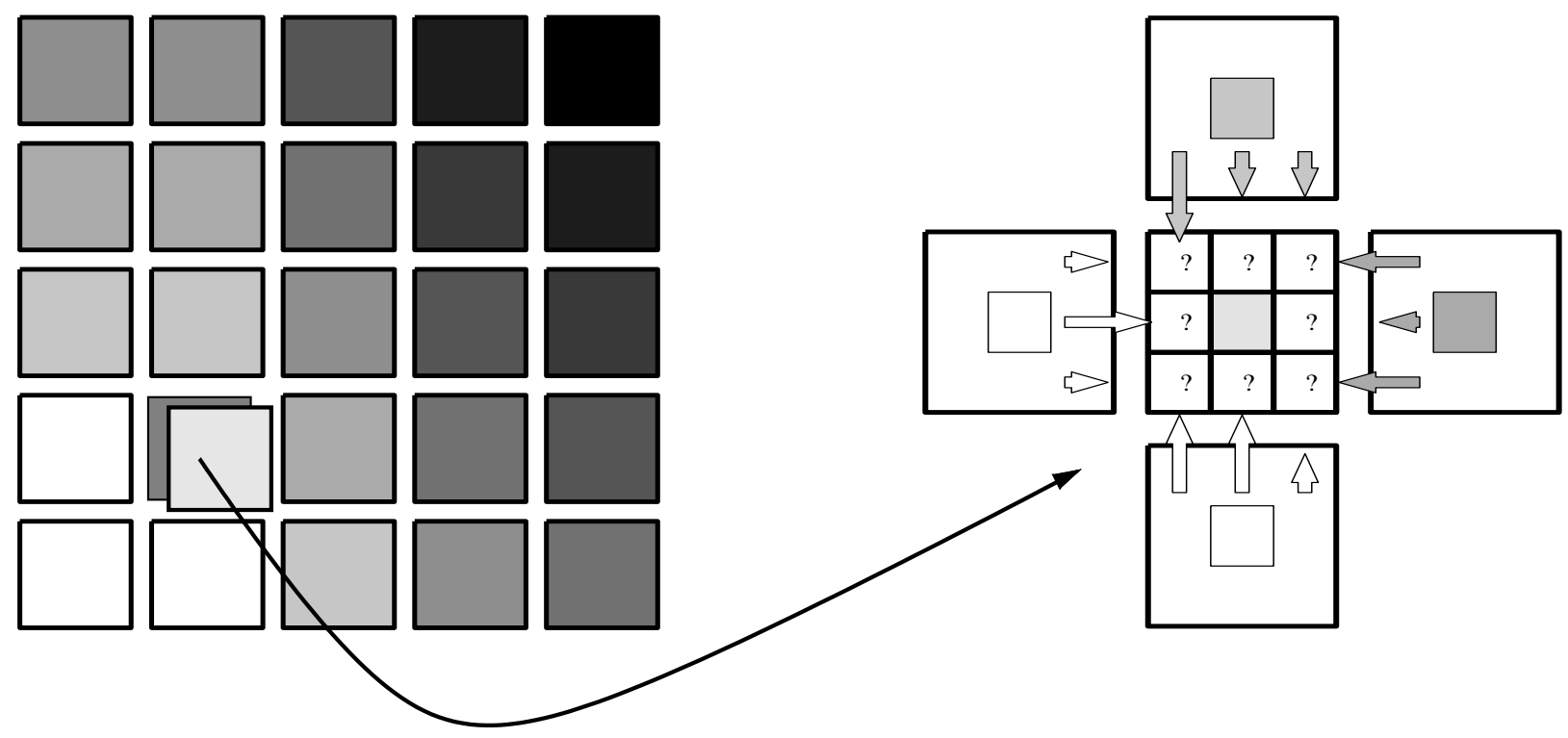

Figure 4.5: Computation of the fine grid pressure field using the pressure of the coarse grid and the fluxes coming from the neighboring coarse grid blocks. The fluxes are computed using Darcy's law and are weighted by the inter-blocks transmissivities.

The pressure on the fine grid is obtained from the former step by solving the pressure equation on each coarse grid block. Let us consider a particular coarse grid block. It contains $m$ fine grid blocks whose pressures will be derived from Eq. 4.7 using the following boundary conditions: the pressure at the central fine grid block is assumed known and equal to the pressure of the coarse grid block. Furthermore, the fluxes at the boundaries of the fine grid are assumed to be equal to $q^{c}$ (the flux computed from the coarse pressure solution using Darcy's law) weighted by the inter-block transmissivities $T_{\bullet, \bullet \pm \frac{1}{2}}$ [214]. For instance, the fine grid flux in the $x$ direction for a particular grid block $(i, j, k)$ located on the right boundary is:

$$
q_{x, i}^{f}=q_{x}^{c} \times \frac{T_{x, i+\frac{1}{2}, j, k}}{\sum_{l_{j}, l_{k}} T_{x, i+\frac{1}{2}, l_{j}, l_{k}}}
$$

In the sum, $\left(l_{j}, l_{k}\right)$ are the indices in the $y$ and $z$ directions of the grid blocks located on the boundary. The upscaling step is described in Figure 4.5.

For coarse grid blocks perforated by a well, we modify slightly the above algorithm. The fluxes along each faces are still considered to be computed with Eq. 4.15. We will require that the pressures at the grid blocks perforated by the well are defined such that the rates are globally the same on both the fine grid and the coarse grid. Let $q^{c, w}$ be the rate flowing from the well from a particular coarse grid block containing $n_{l}$ layers. We assume that the well rate coming from 
each perforated layer $l$ is equal to $q^{c, w}$ weighted by the well transmissivity of the layer $T_{l}^{w}$ which is defined in Peaceman's well model $[170,5]$. We thus have:

$$
q_{l}^{f, w}=\frac{T_{l}^{w}}{\sum_{i=1}^{n_{l}} T_{i}^{w}} \times q^{c, w}
$$

$q_{l}^{f, w}$ is related to the well pressure at the top well grid block completion $\left(k^{*}\right), P_{k^{*}}^{w}$ :

$$
q_{l}^{f, w}=T_{l}^{w}\left(P_{k^{*}}^{w}-P_{l}+\frac{1}{2} \sum_{i=k^{*}+1}^{l}\left(\gamma_{i-1}+\gamma_{i}\right)\left(D_{i}-D_{i-1}\right)\right)
$$

where the wellbore specific gravity is defined by:

$$
\gamma_{i}=\frac{\lambda_{g, i}}{\lambda_{t, i}}
$$

Using Eq. 4.16 and Eq. 4.17 we obtain the pressure of the fine grid block:

$$
P_{l}=P_{k^{*}}^{w}-\frac{q^{c, w}}{\sum_{i=1}^{n_{l}} T_{i}^{w}}+\sum_{i=k^{*}+1}^{l}\left(\gamma_{i-1}+\gamma_{i}\right)\left(D_{i}-D_{i-1}\right)
$$

This method ensures that the flow will preferentially exit the well through high transmissivity layers.

Once the pressure is defined on the fine grid, we compute the velocity field in order to trace streamlines. To ensure continuity of the fluxes, the velocity field is computed from the fine grid pressure within each coarse grid block volume, and from the coarse fluxes on the boundaries, Eq. 4.15, that were used in the pressure solution on the fine grid. Once the streamlines are defined, the saturation is moved forward in time as in a standard streamline method. The pressure field and streamlines are periodically recomputed during the displacement. The saturation is always defined on the fine grid and is used in the computation of $\mathbf{T}^{c}$ and $\mathbf{G}$ for the coarse grid.

Figure 4.6 summarizes the different steps of the nested grid method. Note that only the steps (2-4) are different from the classical streamline method where they are replaced by a pressure solve over the entire fine grid.

\subsection{CPU Cost Evaluation for the Nested Gridding Method}

We will present in this section a comparison of the CPU cost for the conventional streamlinebased simulation against our new nested gridding approach. We compare the CPU time needed to solve Eq. 4.7 for both methods. The other steps in the simulation are similar for the two methods. The CPU time needed to solve directly the pressure equation on the fine grid is:

$$
t^{(\text {direct })}=t(N \times m)
$$

In the nested gridding method, the time $t^{\text {(nested) }}$ required to solve the pressure equation in $d=2,3$ dimensions is the sum of three terms:

$$
\begin{aligned}
t^{(\text {nested })} & =\left(d+1_{g}\right) N t(m) \\
& +t(N) \\
& +N t(m)
\end{aligned}
$$




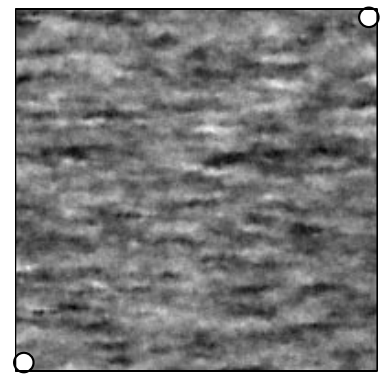

(a) Fine grid transmissivity map

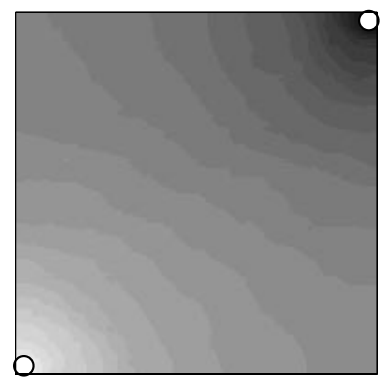

(d) Pressure on the fine grid

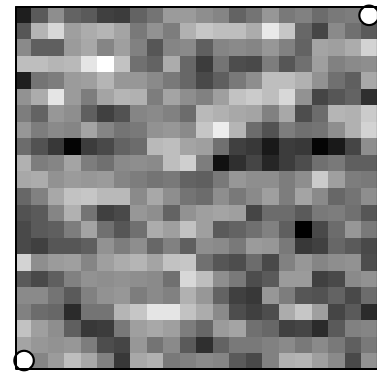

(b) Coarse grid transmissivity map

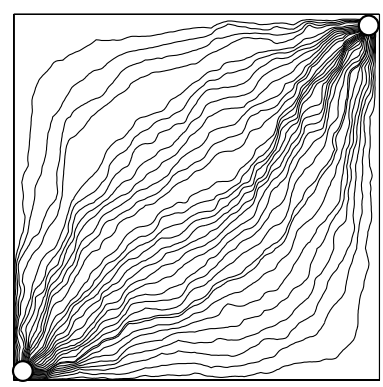

(e) Streamlines on the fine grid

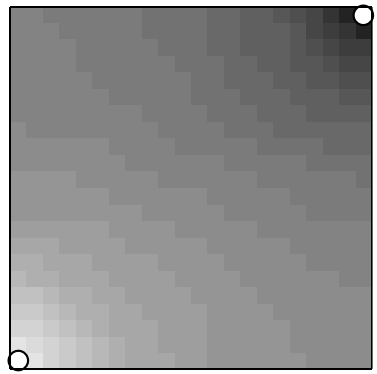

(c) Pressure on the coarse grid

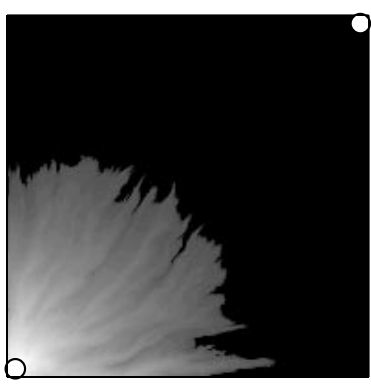

(f) Saturation on the fine grid

Figure 4.6: Description of the different steps of the nested gridding method. We first consider a fine description of the reservoir (a). The transmissivities are then upscaled on a coarse scale (b) and a pressure solution on the coarse grid is computed (c). The next step is the computation of the pressure on the fine grid (d) which leads to the velocity field. The streamlines are traced from the injector to the producer $(e)$ and saturation is moved using the classical streamline method $(f)$. 
The first term Eq. 4.21, represents the amount of time necessary to solve the $N$ small linear systems needed to perform the upscaling in $d$ directions of the transmissivities and the upscaling of the gravity transmissivities assuming that the time required to get the solution is $t(m)$. $1_{g}$ is equal to 1 or 0 depending on whether or not gravity is considered. The second term, Eq. 4.22, represents the time needed to solve the pressure field on the coarse scale and the last term Eq. 4.23 is the time needed to solve compute the pressure inside all the different coarse grid blocks. Adding these terms we obtain:

$$
t^{(\text {nested })}=\left(d+1_{g}+1\right) N t(m)+t(N) \quad \text { with } d=2,3
$$

For the upscaling step, for $3 D$ problems, three or four linear systems have to be solved to compute the effective transmissivities in the three directions and the gravity transmissivities. But, since the same transmissivity matrix, is used three times and is stored in memory, the required time is less than 3 or $4 N t(m)$.

The nested gridding method requires us to solve multiple linear systems whose sizes are highly different: typically for the upscaling step and the computation of the fine grid pressure, the system will contain $10-100$ unknowns, whereas for the computation of the coarse grid pressure the number of unknowns is $10^{4}-10^{5}$. One key issue of the nested gridding method is its ability to use the most appropriate solver at each scale to obtain the lowest CPU time cost. In the next section different solvers are presented and tested. We will see that their performances are sensitive to the number of unknowns as well as to the available computer memory.

\subsection{CPU Requirements of Different Pressure Solvers}

The pressure is solved from Eq. 4.7. Typically, considering all the grid blocks, we obtain a banded linear system of equations. The bandwidth depends on the total number of grid blocks and on the numbering of the grid blocks. Figure 4.7 and Figure 4.8 represent the shape of the matrix formed with the transmissivity of the reservoir in $3 D$ and $2 D$ (only nonzero elements are represented). Adding well constraints leads to additional off-diagonal terms.
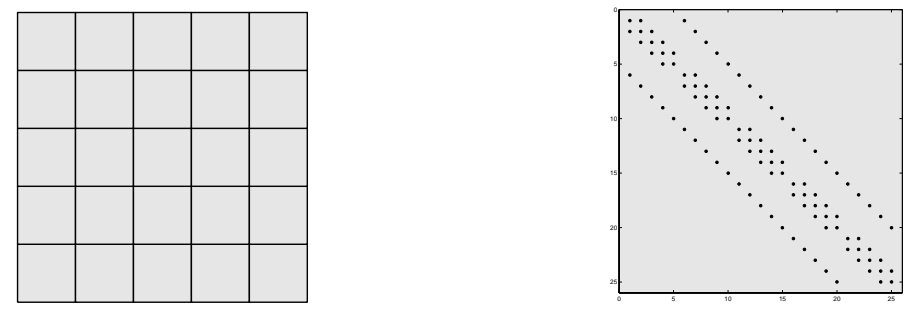

Figure 4.7: 2Dgrid with $n_{x}=5, n_{y}=5$ and the corresponding transmissivity sparse matrix. Only nonzero elements are represented.

We will use three different public domain solvers:

- a band solver DGBSV from the $L A P A C K$ library [4].

- an iterative solver from the ITPACK library [116].

- a multigrid solver $A M G$ [202].

The performance and memory requirements of these two solvers are very different. For the band solver, the data are stored in an $N_{b} \times N_{t}$ array, where $N_{b}$ and $N_{t}$ represent respectively the bandwidth 

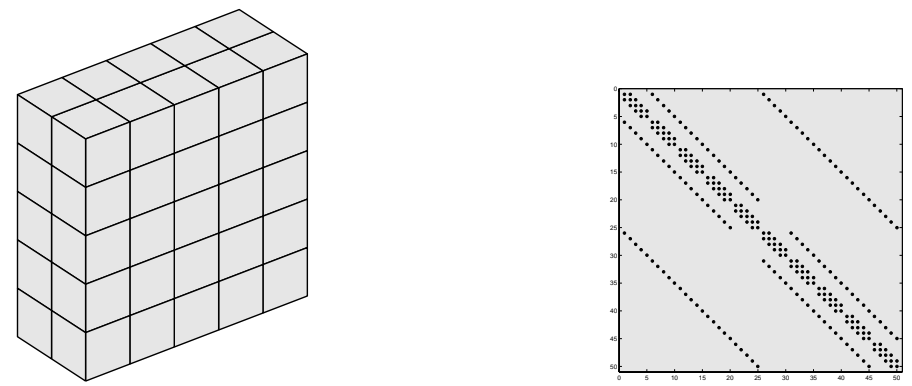

Figure 4.8: $3 D$ grid with $n_{x}=2, n_{y}=5$ and $n_{z}=5$ and the corresponding sparse matrix. Only nonzero elements are represented.

and the total number of grid blocks. The storage space increases quickly with the system size and makes this method only suitable for problems containing less than 30,000 grid blocks in $2 D$ using a DEC alpha workstation with 512MB RAM. The iterative solver and the multigrid solver both use a storage which is proportional to a weighted sum of the number of nonzero elements and the total number of grid blocks, $N_{t}$. The iterative solver takes also advantage of the symmetric properties of the transmissivity matrix. Only one half of the nonzero elements are stored. A 500,000 grid block problem was the largest case we could run with our computer using the multigrid solver, whereas problems with up to two million grid blocks were possible using the iterative solver.

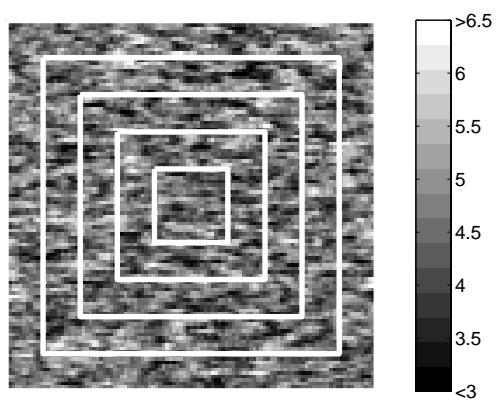

(a)

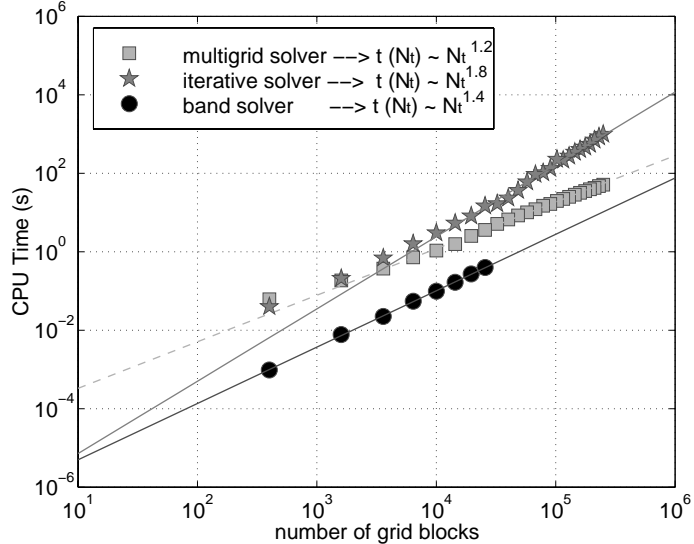

(b)

Figure 4.9: Heterogeneous permeability fields (a), in a log-scale, used to compare the CPU time needed to solve the pressure. There are all extracted from a $500 \times 500$ permeability field. (b) is the $\mathrm{CPU}$ time comparison for a pressure solve using different solvers for $2 D$ problems.

We now compare the CPU cost of different solvers for $2 D$ problems. We consider a permeability field $(500 \times 500$ grid blocks $)$ generated using a sequential gaussian simulation [41]. The pressure equation is solved for permeability fields extracted from it with a prescribed pressure on two opposing faces and no flux boundary on the two other faces. The construction of the different permeability fields is depicted in Figure 4.9(a). The CPU time needed for the pressure solve is plotted as a function of the total number of grid blocks $N_{t}$ in Figure 4.9(b) for the three available solvers.

For a sufficiently large number of grid blocks the time for a pressure solve using the multigrid 
Table 4.1: CPU time (in $s$ ) and memory (in MB) comparisons for a pressure solve on $3 D$ permeability fields of varying size.

\begin{tabular}{ccccc}
\hline Dimension $\left(n_{x} n_{y} n_{z}\right)$ & \multicolumn{2}{c}{ ITPACK } & \multicolumn{2}{c}{ AMG } \\
\cline { 2 - 5 } & RAM & CPU & RAM & CPU \\
\hline $30^{3}$ & 11 & 4.4 & 27 & 4.1 \\
\hline $50^{3}$ & 35 & 52 & 110 & 63 \\
\hline $70^{3}$ & 86 & 250 & 294 & 275 \\
\hline $90^{3}$ & 178 & 703 & - & - \\
\hline
\end{tabular}

solver scales as $N_{t}^{1.2}$, as $N_{t}^{1.8}$ for the iterative solver and as $N_{t}^{1.4}$ for the band solver. The band solver uses less CPU time than the two other methods. The drawback is that the memory requirements are so high that it can be use for only relatively small problems, such as the computation of the pressure within each coarse grid block during the upscaling step. The multigrid solver is always faster than the iterative solver when considering more than ten thousand grid blocks for $2 D$ problems.

In the nested gridding method, the band solver is used for the upscaling step as well as for the computation of the pressure back to the fine grid. The pressure on the coarse grid is computed using the multigrid method, or the iterative method if the memory requirements are too high. As an example, we can evaluate a first approximation of the speed-up factor for $600 \times 600$ grid blocks. The time required to solve the pressure equation directly on the fine grid, using the $A M G$ solver, is about $80 \mathrm{~s}$. For the nested gridding approach, using a $3 \times 3$ upscaling factor, the time to solve the pressure field from Eq. 4.24 is:

$$
\begin{aligned}
t^{(\text {nested })}(600 * 600) & = & 3 * 200 \times 200 \times t(9)+t(200 * 200) \\
& = & 3 * 4.210^{-6} \times 40000+6.2 \\
& = & 6.7 \mathrm{~s}
\end{aligned}
$$

The speed-up factor is around 10 for this example.

Table 4.1 shows some comparisons of the solvers for $3 D$ problems. Here, the iterative solver is as fast as the multigrid solver. Hence, the iterative solver will be used to solve the pressure field on the coarse scale since the memory requirements are lower. As an evaluation of the speed-up factor, let us consider a permeability field containing $90 \times 90 \times 90$ grid blocks with an upscaling factor $(3 \times 3 \times 1)$ (The effective transmissivity in the $z$ direction is simply the arithmetic mean of the fine grid transmissivities and the factor $(d+1)$ in Eq. 4.24 is actually close to 3$)$. We neglect gravity effects. Using Table 4.1 and Figure 4.9(b) (since each pressure solve for a single coarse grid block is a $2 D$ problem), the time needed for the upscaling method is $20 \mathrm{~s}$ leading to a speed-up factor equal to 35. In all these evaluations, the construction of the different transmissivity matrices on the coarse and on the fine scale and the different data manipulations involved by the nested gridding method were not considered. Therefore, the speed-up factors may be overestimated.

\subsection{Results}

In this section, we will compare the results provided by the streamline method with the new nested gridding method. The streamline simulator is 3DSL [10, 204], and the subroutines for the pressure solve have been updated for the new nested gridding method.

The permeability fields used in this part are generated with a indicator algorithm (SISIM) from the GSLIB library [41]. These fields are characterized by three $(3 D)$ or two $(2 D)$ correlation lengths $l_{x}, l_{y}, l_{z}$ and by the different value of the permeability facies. The cases study are waterflood problems with two wells, a producer and an injector with prescribed pressure. The fluid viscosity 
ratio between oil and water is $\mu_{o} / \mu_{w}=10$. The relative permeability curves are quadratic functions of the saturations. We will compare saturation maps and recovery curves for three methods:

1. The nested gridding method.

2. Streamline simulation on the fine grid.

3. Streamline simulation on the coarse grid where the absolute permeability is upscaled as described in this chapter. There is no upscaling of relative permeability. We call this the upscaling method.

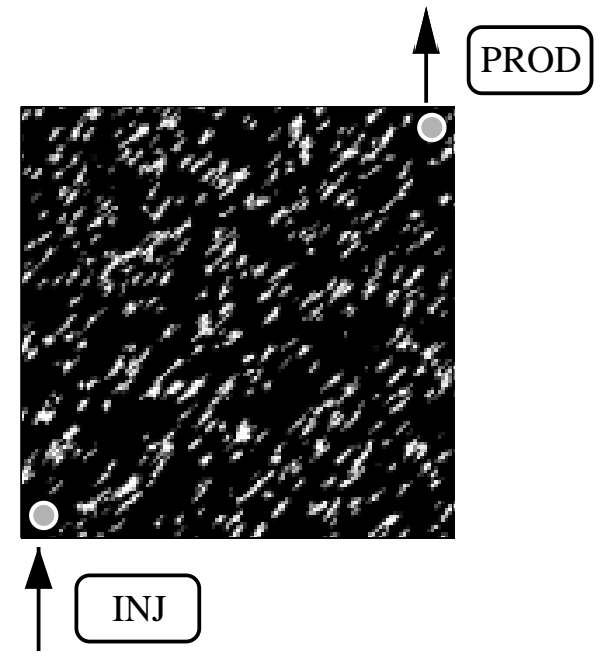

Figure 4.10: Permeability field containing $270 \times 270$ grid blocks with two facies. Permeability: $k$ (black) $=100 \mathrm{mD}$ and $k$ (white) $=0.1 \mathrm{mD}$. Proportion of black facies: $90 \%$. Correlation length: 10 grid blocks in the $x$ and $y$ directions with an anisotropy angle of $45^{\circ}$

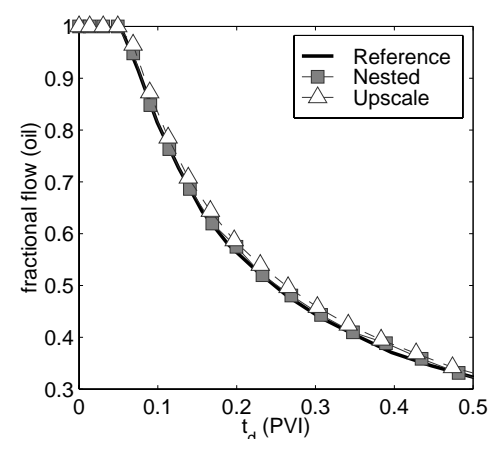

(a) $(3 \times 3)$

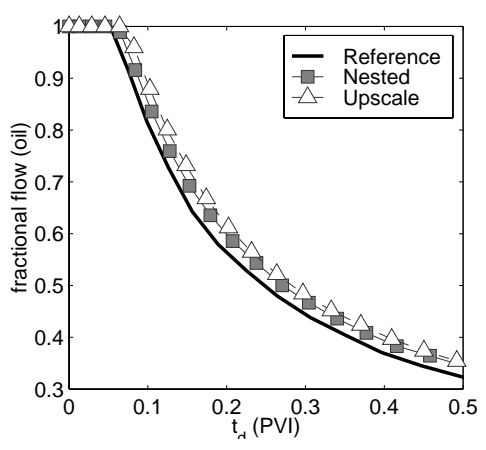

(b) $(9 \times 9)$

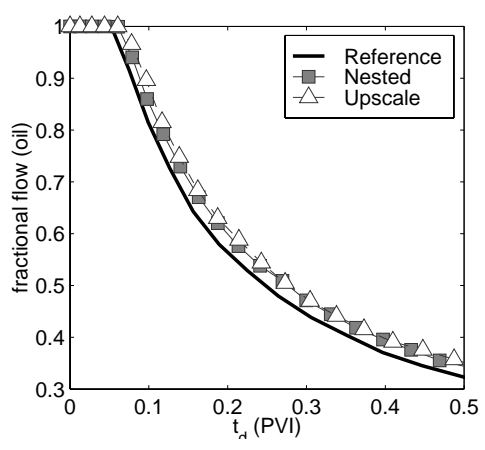

(c) $(27 \times 27)$

Figure 4.11: Fractional flow of oil at the producer for different upscaling factors and for the three methods (fine grid, nested gridding and upscaling). Case RES1.

\subsubsection{Numerical Results for Two-Dimensional Reservoirs}

Figure 4.10 represents a permeability field RES1 containing two facies with $n_{x}=n_{y}=270$ grid blocks, $l_{c x}=l_{c y}=10$ (in grid block units). The black facies represents grid blocks with a 


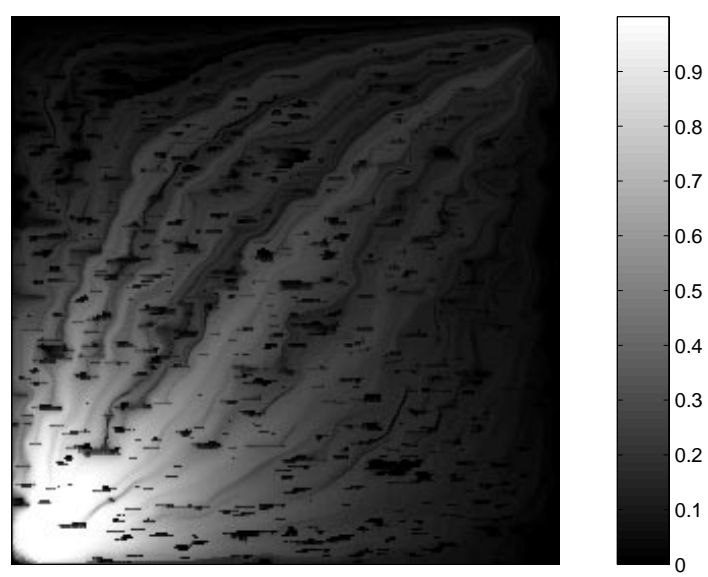

(a) Fine grid simulation

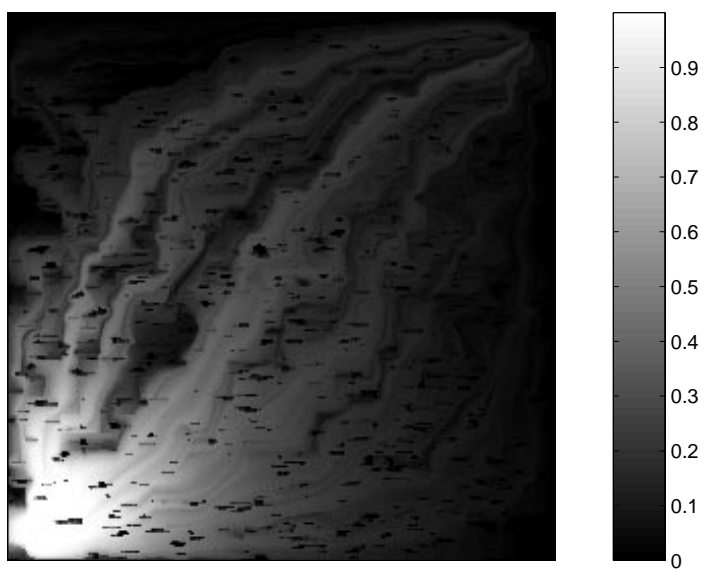

(b) Nested gridding method

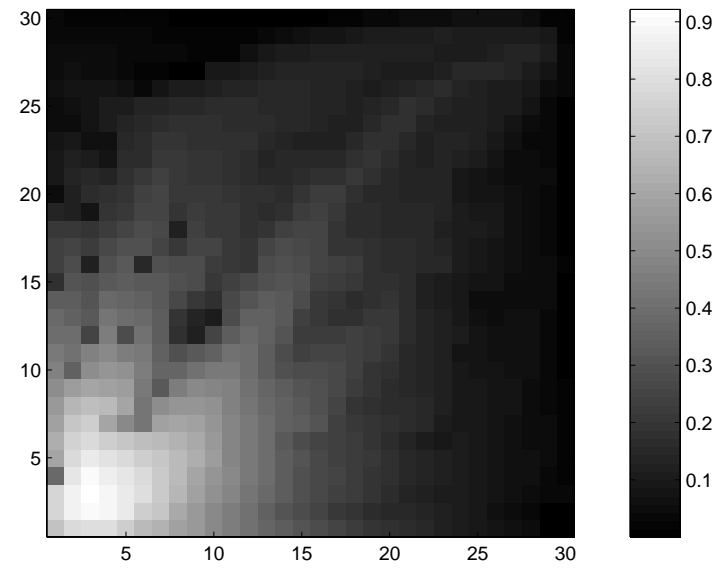

(c) Upscaling method

Figure 4.12: Water saturation maps from the fine grid simulation (a), from the nested gridding simulation (b) and from the simulation on the upscaled absolute permeabilities (c) for 0.35 PVI. The upscaling factor is $9 \times 9$. Case $R E S 1$. 
permeability equal to $100 \mathrm{mD}$. The white facies permeability is $0.1 \mathrm{mD}$. The proportion of the black facies is $90 \%$. Water is injected from the lower-left corner well and the fractional flow of oil is observed at the producer (upper-right corner). Results are shown in Figure 4.11 for the three methods and for three upscaling factors (each coarse grid blocks contains $3 \times 3,9 \times 9$ or $27 \times 27$ fine grid blocks). We can see that for the smallest upscaling factors, both the nested gridding and the upscaling give good approximations of the reference fine grid simulation. When we coarsen, the difference increases between the fine grid simulation and the two approximate methods. The nested gridding is slightly better than upscaling. The saturation maps for the three methods are shown in Figure 4.6 for a $9 \times 9$ upscaling factor for 0.35 PVI. Fine grid details are captured by the nested gridding methods, while only the main features of the flow are detected by upscaling.

We now consider a more demanding problem with greater channeling. Figure 4.13 represents a

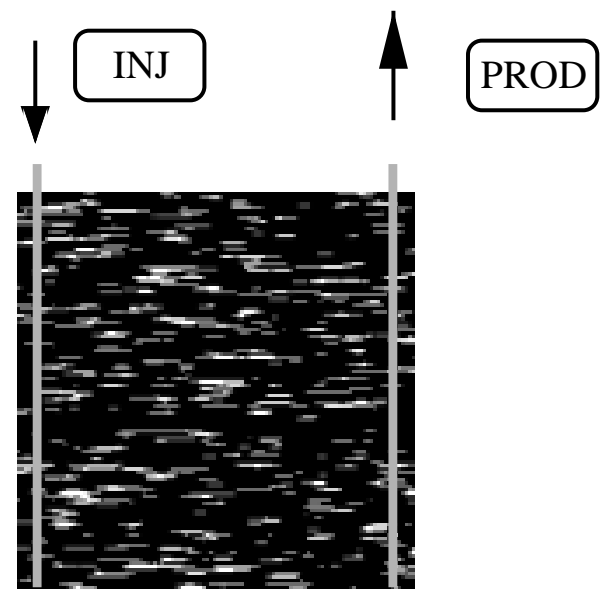

Figure 4.13: Permeability field containing $270 \times 270$ grid blocks with two facies. Permeability: $k$ (black $)=100 \mathrm{mD}$ and $k$ (white) $=0.1 \mathrm{mD}$. Proportion of black facies: $90 \%$. Correlation length: 20 grid blocks in the $x$ and 2 grid blocks in the $y$ direction.

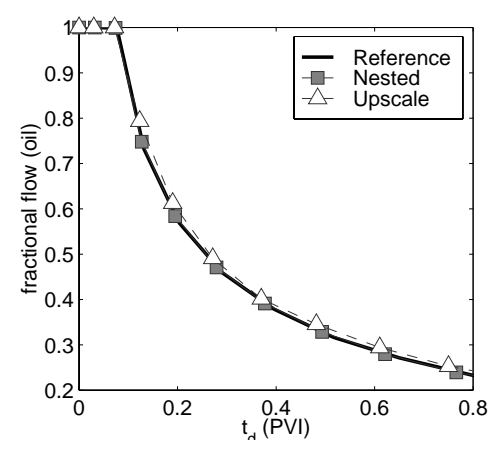

(a) $(3 \times 3)$

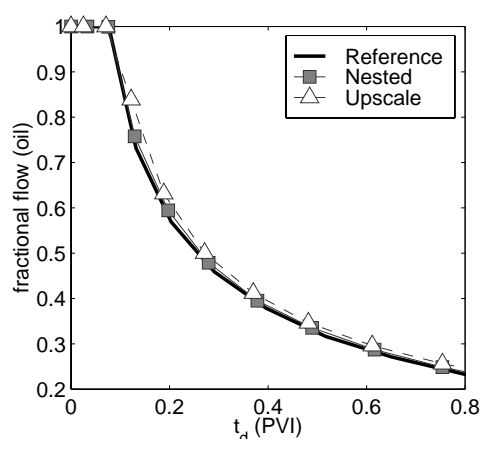

(b) $(9 \times 9)$

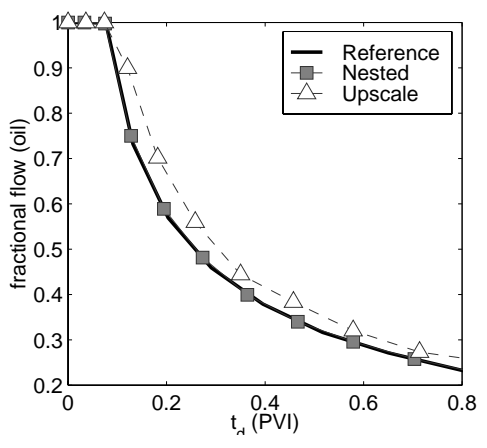

(c) $(27 \times 27)$

Figure 4.14: Fractional flow of oil at the producer for different upscaling factors and for the three methods (fine grid, nested gridding and upscaling). Case RES2

layered permeability field on the $(x, z)$ plane containing two facies with $n_{x}=n_{y}=270$ grid blocks, $l_{c x}=20 l_{c z}=2$ (in grid block units). The proportions and permeability of the two facies are similar to the case RES1. 


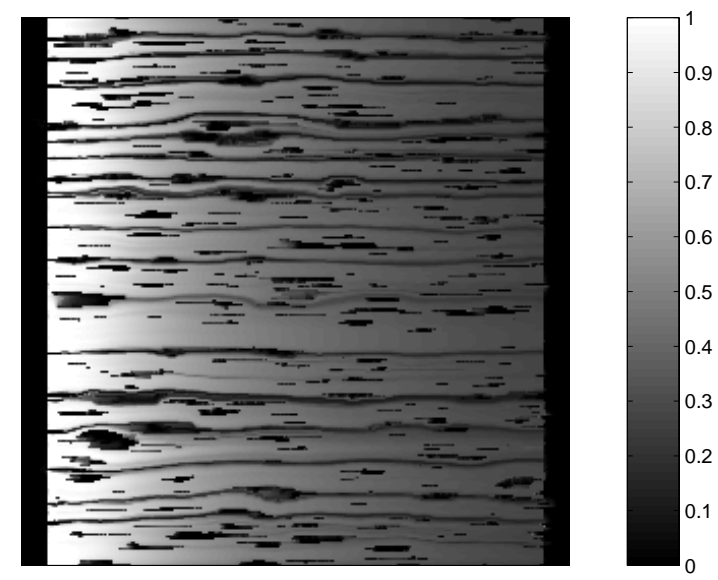

(a) Fine grid simulation

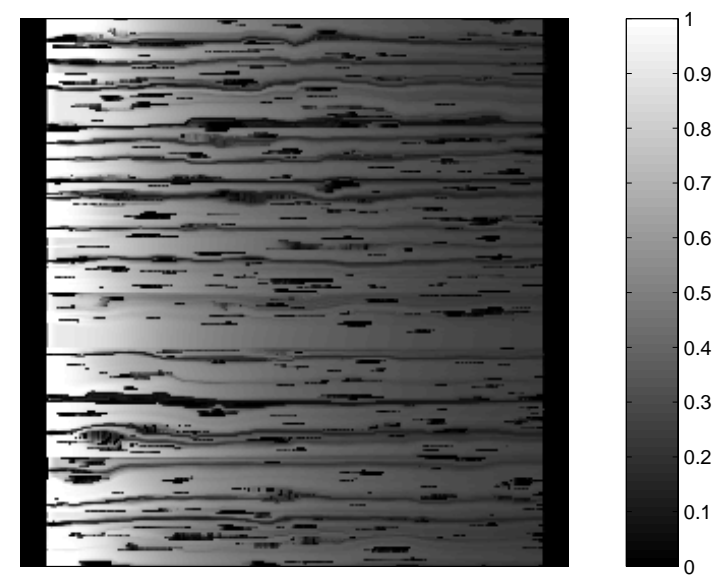

(b) Nested gridding method

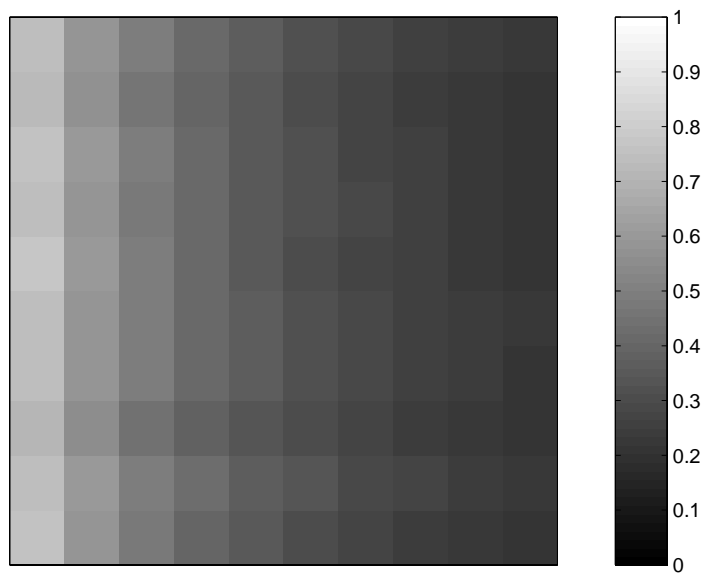

(c) Upscaling method

Figure 4.15: Water saturation maps from the fine grid simulation (a), from the nested gridding simulation (b) and from the simulation on the upscaled absolute permeabilities (c) for $0.8 P V I$. The upscaling factor is $27 \times 27$. Case $R E S 2$ 
Table 4.2: Speed-up factor and memory requirements of the nested grid method for a pressure solve and for the whole simulation on the two $2 D$ examples presented in the chapter.

\begin{tabular}{cccccc}
\hline & Upscaling factor & \multicolumn{2}{c}{ Speed-up factor } & \multicolumn{2}{c}{ Memory } \\
& & $P$ solve & sim & RAM & $\%$ saved \\
\multirow{2}{*}{ RES1 } & $3 \times 3$ & 3.8 & 1.2 & 19 & 61 \\
\cline { 2 - 6 } & $9 \times 9$ & 4.3 & 1.5 & 13 & 73 \\
\hline \multirow{2}{*}{ RES2 } & $3 \times 3$ & 5.6 & 2.1 & 19 & 61 \\
\cline { 2 - 6 } & $9 \times 9$ & 6.2 & 1.6 & 13 & 73 \\
\hline
\end{tabular}

Water is injected from the left well and oil is produced from the right. A constant pressure difference is prescribed between the two wells which are fully penetrating. Gravity is neglected. Production curves are shown in Figure 4.14 for the three methods and for three upscaling factors $(3 \times 3,9 \times 9$ or $27 \times 27$ fine grid blocks). We can see that as the grid is coarsened the results from upscaling get worse. In contrast, nested gridding gives results almost identical to fine grid simulation, even for high upscaling factors. To understand better this behavior we present in Figure 4.14 three maps representing the saturation for $0.7 \mathrm{PVI}$ derived from the three different simulations with an upscaling factor equal to $27 \times 27$. The preferential flow paths induced by the permeability pattern are well represented by the nested gridding method since it retains an explicit description of the saturation distribution on the fine grid. The upscaling method gives poor results with an overly smooth saturation profile. Thus fine grid details are not taken into account.

Table 4.2 shows the CPU time and memory comparisons for the nested gridding method and the classical streamline method. The multigrid solver was used to compute the pressure field for the reference case. The average speed-up factor for the pressure solve is 5 and decreases to 1.5 for the entire simulation. This is because there are no time savings for tracing streamlines and moving saturations, since these steps are performed on the fine grid. Up to $73 \%$ of the memory is saved since we don't have to store the transmissivity matrix related to the fine grid $(N \times m$ grid blocks) but only the transmissivity matrix related to the coarse grid ( $N$ grid blocks) and to the fine grid within a coarse grid block ( $m$ grid blocks).

\subsubsection{Numerical Results for Three-Dimensional Reservoirs}

We will now present a $3 D$ example. We consider a $99 \times 99 \times 99$ permeability field containing two facies. Small low permeability clusters $(0.1 \mathrm{mD})$, characterized by a correlation length of 2 grid blocks in every direction, are embedded into a matrix of higher permeability $(100 \mathrm{mD})$. The proportion of the low permeability clusters is $20 \%$. The permeability map and the well locations are represented in Figure 4.16.

In this $3 D$ example we will show two different results with and without gravity. We define for that purpose a gravity number which compares the relative contribution of the viscous forces over the gravity forces $[63,203]$ :

$$
N_{g}=\frac{\Delta \rho g L^{2}}{\Delta P_{h} H}
$$

where $\Delta \rho$ represents the density difference between water and oil, $L^{2}$ and $\Delta P_{h}$ are the distance and the pressure difference between the two wells and $H$ is the height of the reservoir. We will consider the case where the gravity can be neglected $\left(N_{g}=0\right)$ and the case where the effects are important $\left(N_{g}=2\right)$.

Water is injected in the injector and we observe the oil fractional flow at the producer for $N_{g}=0$ and 2 in Figure 4.17 and Figure 4.18 respectively. Clearly in all the cases, and for 


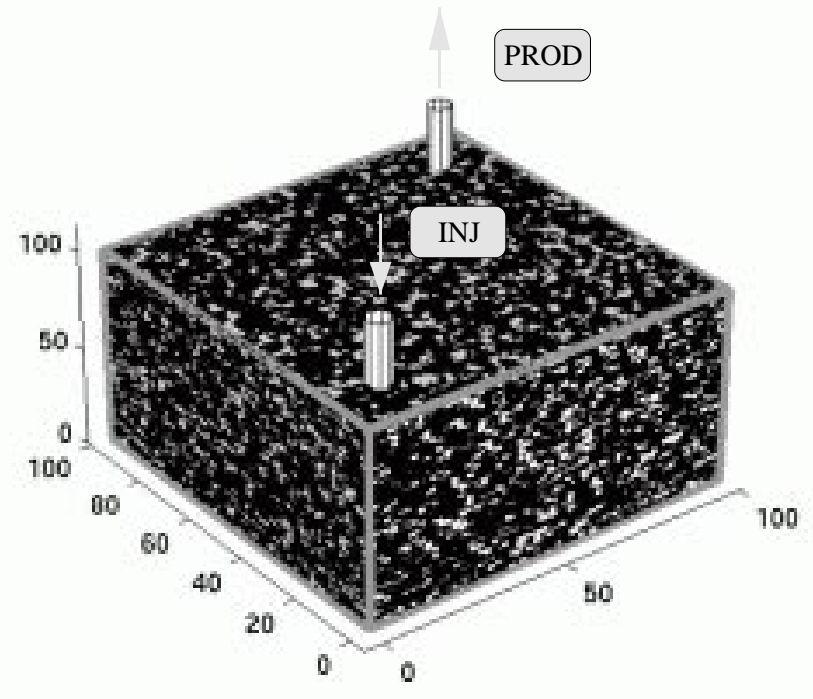

Figure 4.16: Case RES3. $99 \times 99 \times 99$ permeability field. The two wells are located on two opposing corners of the reservoir and they penetrate all the layers. The correlation length is equal to 2 grid blocks in all 3 directions. The permeability of the black facies is $100 \mathrm{mD}$ and $0.1 \mathrm{mD}$ for the white facies.

all the different upscaling factors, the nested gridding is more accurate than the upscaling which overestimated the breakthrough time and the overall oil production.

Figure 4.19 represents a zoom of the saturation maps for the case $R E S 3$ when $N_{g}=0$. The figure shows four slices of the $3 D$ array. Only one third of the reservoir is shown. The upper left corner represents the injector. Once again, we can see that the water front advance and fine grid details are accurately captured by the nested gridding method. The upscaling method gives a smoothed representation of the flow pattern.

Finally, saturation maps for the entire reservoir are presented for the case where $N_{g}=2$ (Figure 4.20), which shows clearly the important effects of gravity which segregates the two fluids. Here, water which is denser than the oil preferentially moves to the bottom of the reservoir. Gravity effects are also well accounted by our method.

Table 4.3 compares the CPU time and memory requirements of the nested gridding simulation with the fine grid simulation for the $3 D$ examples. The multigrid solver was used to compute the pressure field for the reference case. In the third column, the numbers shown in parenthesis correspond to the case where gravity is considered. For the pressure solve, the speed-up factor can go up to 37 for an upscaling factor of 3 in all directions. Note that the maximum speed-up factor does not correspond to the maximum upscaling factor. The overall speed-up factor is only 8.5. This is because we have only modified the pressure solve. When adding gravity, the overall speedup factor is approximately halved mainly due to an additional step used to solve the saturation equation [10].

We have also performed CPU time comparisons between the nested gridding and the upscaling method for the $3 D$ examples. For a pressure solve, the upscaling method is about 5-7 times faster than nested gridding. For the whole simulation upscaling is between 30-60 times faster than nested gridding. 


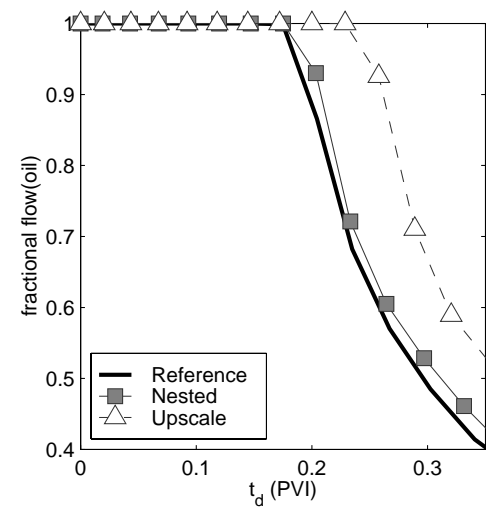

(a) $(3 \times 3 \times 1)$

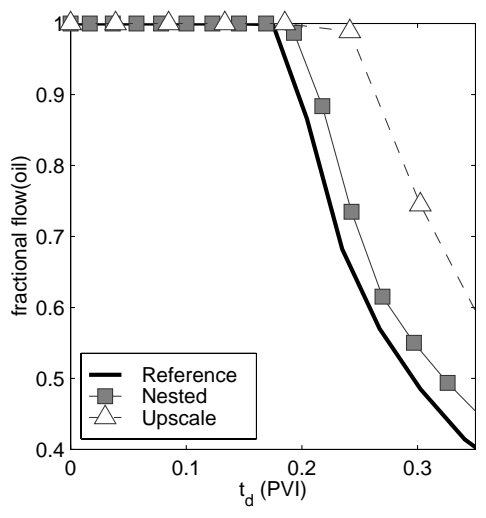

(b) $(3 \times 3 \times 3)$

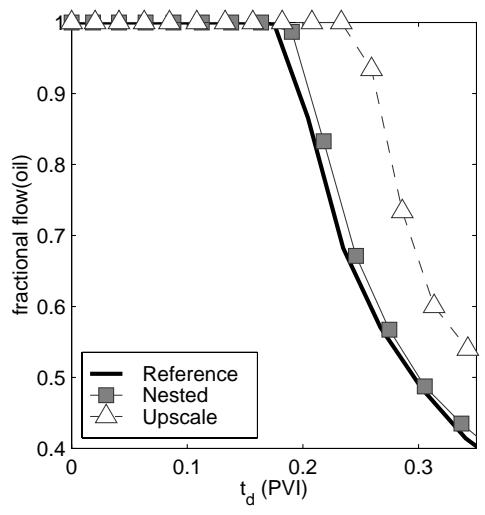

(c) $(9 \times 9 \times 3)$

Figure 4.17: Fractional flow of oil at the producer for different upscaling factors and for the three methods (fine grid, nested gridding and upscaling) for the case RES3. The gravity number is set to $N_{g}=0$.

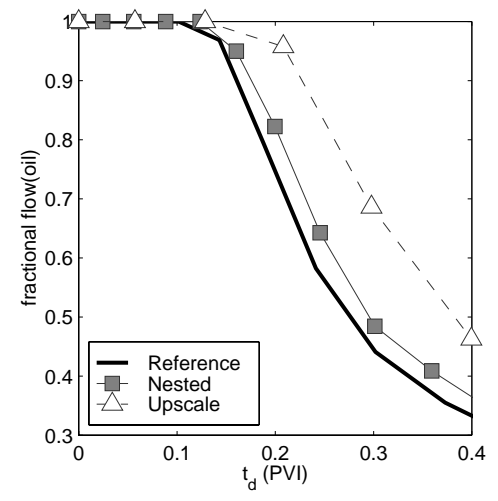

(a) $(3 \times 3 \times 1)$

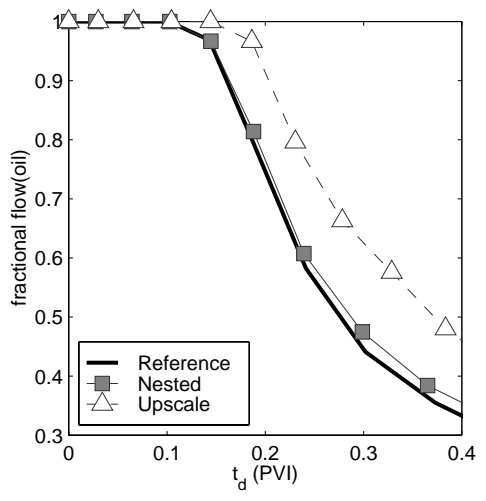

(b) $(3 \times 3 \times 3)$

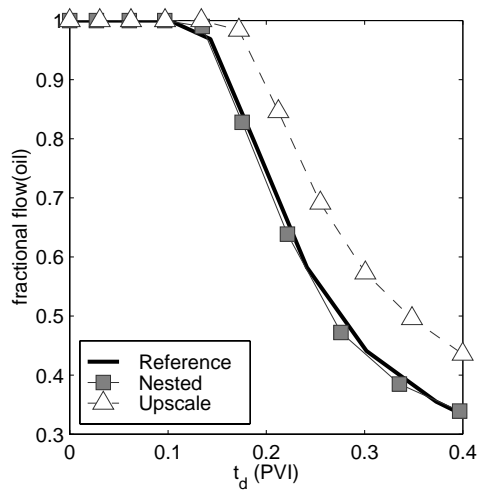

(c) $(9 \times 9 \times 3)$

Figure 4.18: Fractional flow of oil at the producer for different upscaling factors and for the three methods (fine grid, nested gridding and upscaling) for the case RES3. The gravity number is set to $N_{g}=2$. 


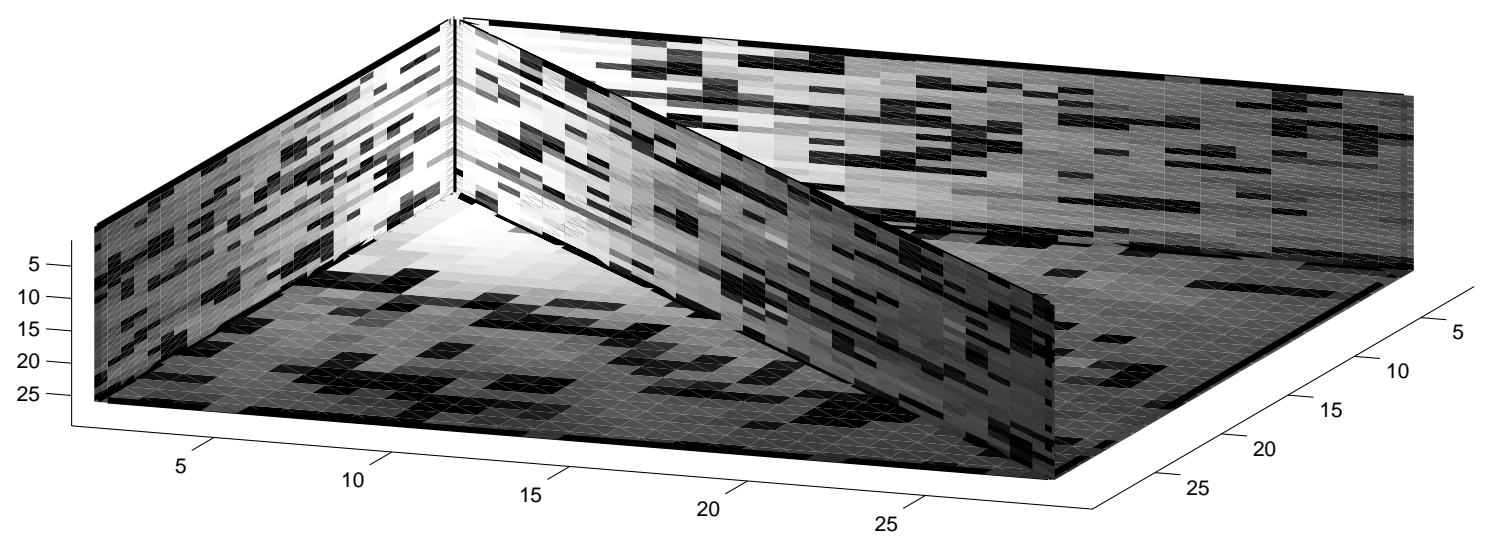

(a) Fine Grid

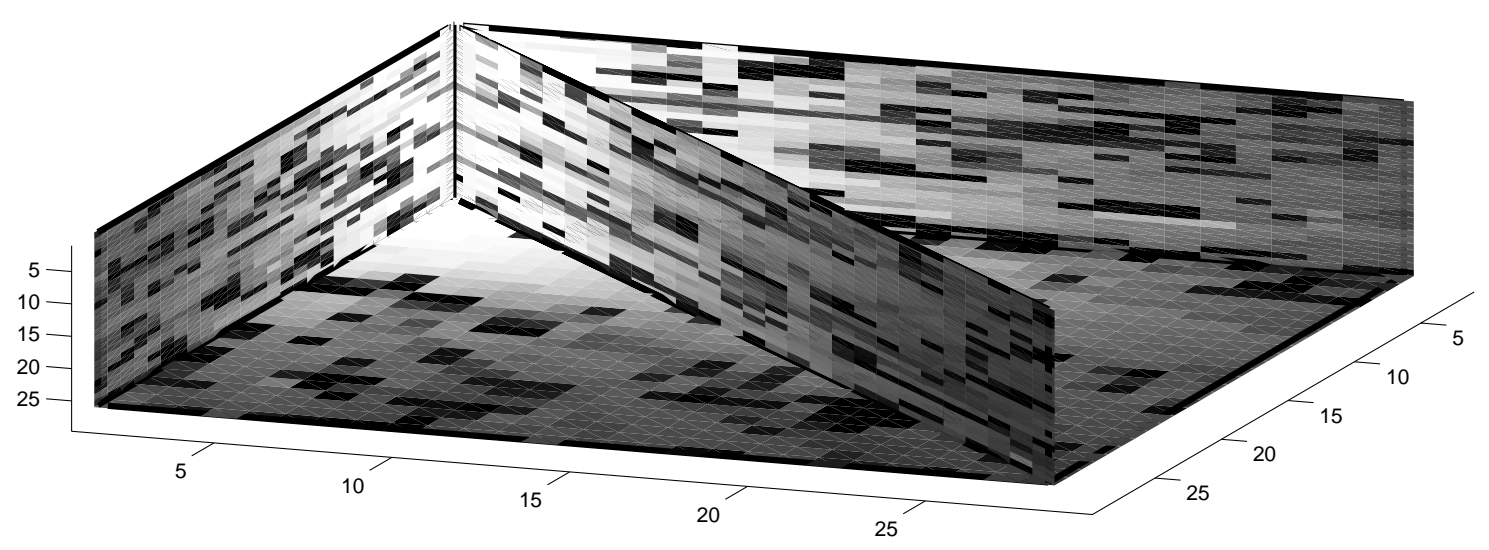

(b) Nested Gridding

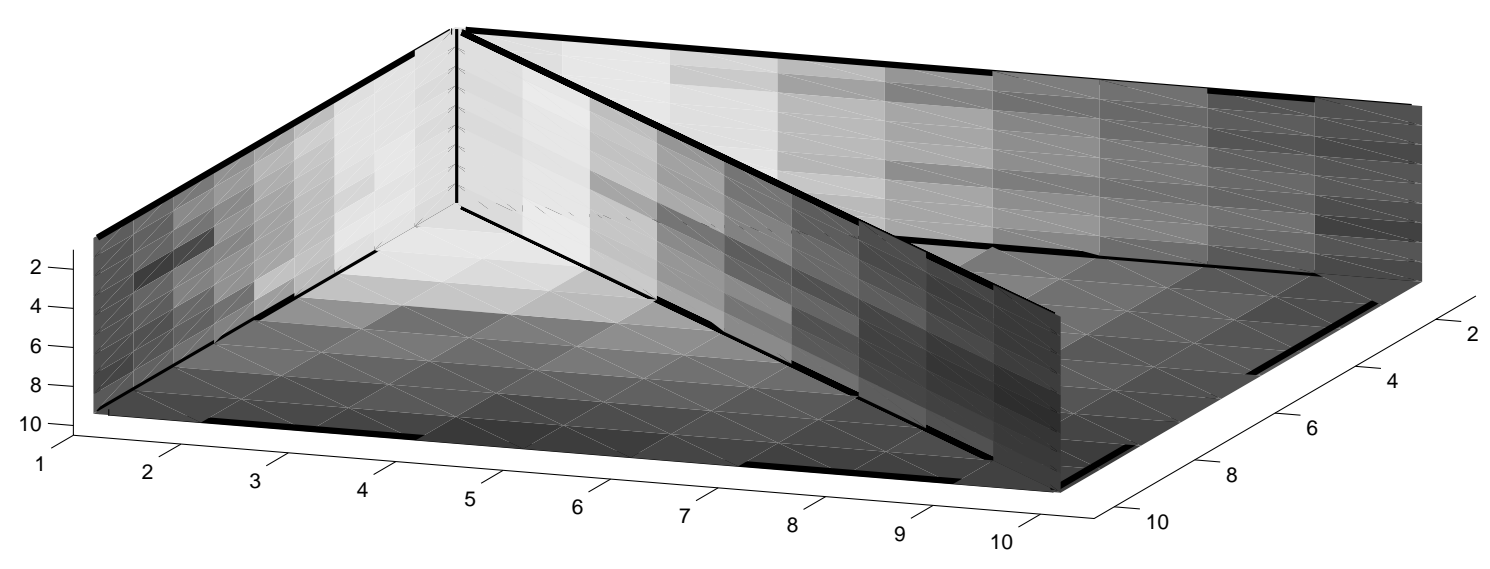

(c) Upscaling

Figure 4.19: Saturation maps for 0.15 PVI using the three methods (fine grid, nested gridding and upscaling) with an upscaling factor equal to $(3 \times 3 \times 3)$. The gravity number is set to $N_{g}=0$ 


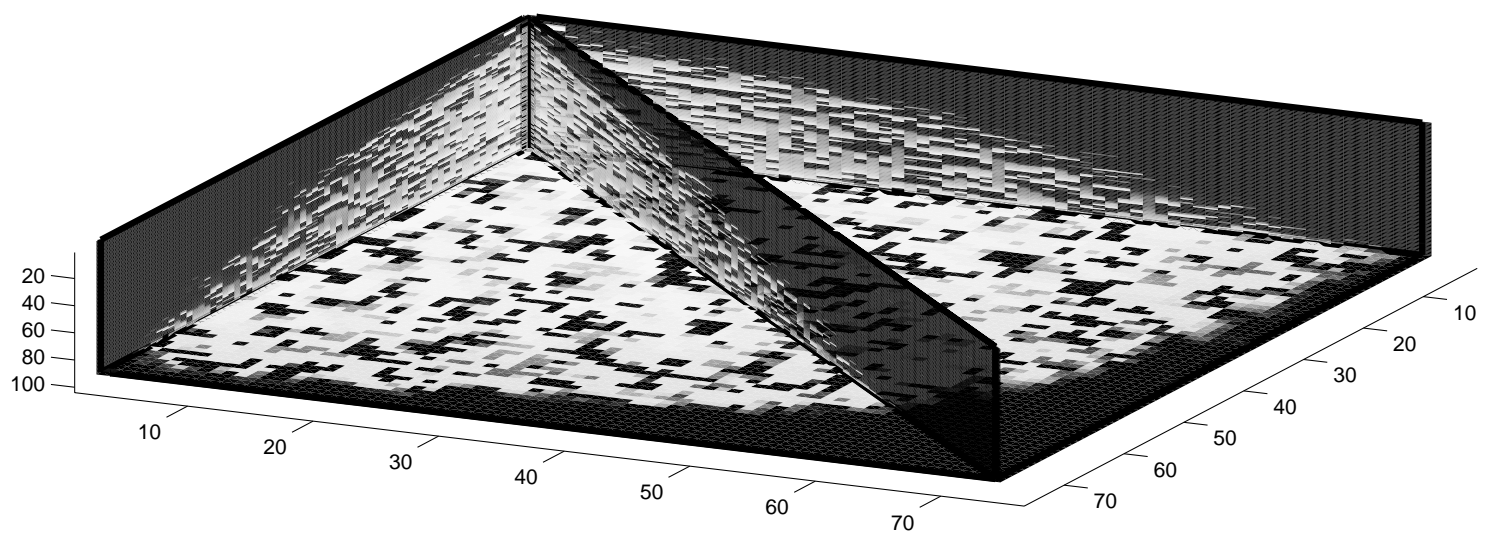

(a) Fine Grid

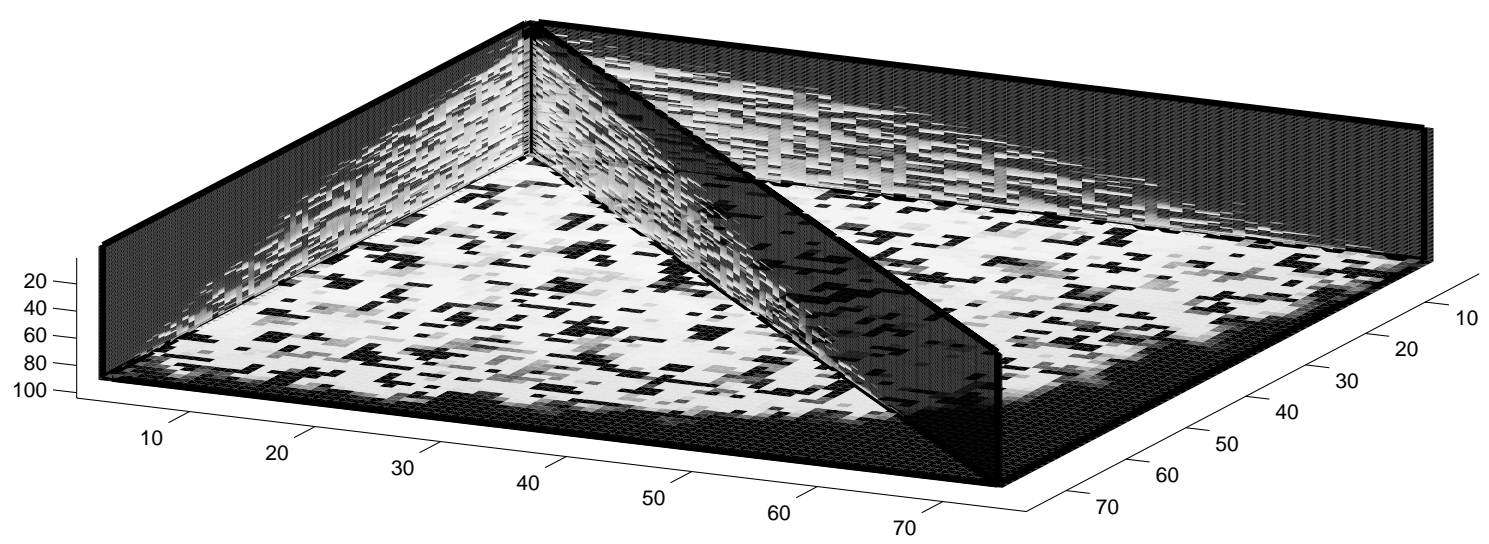

(b) Nested Gridding

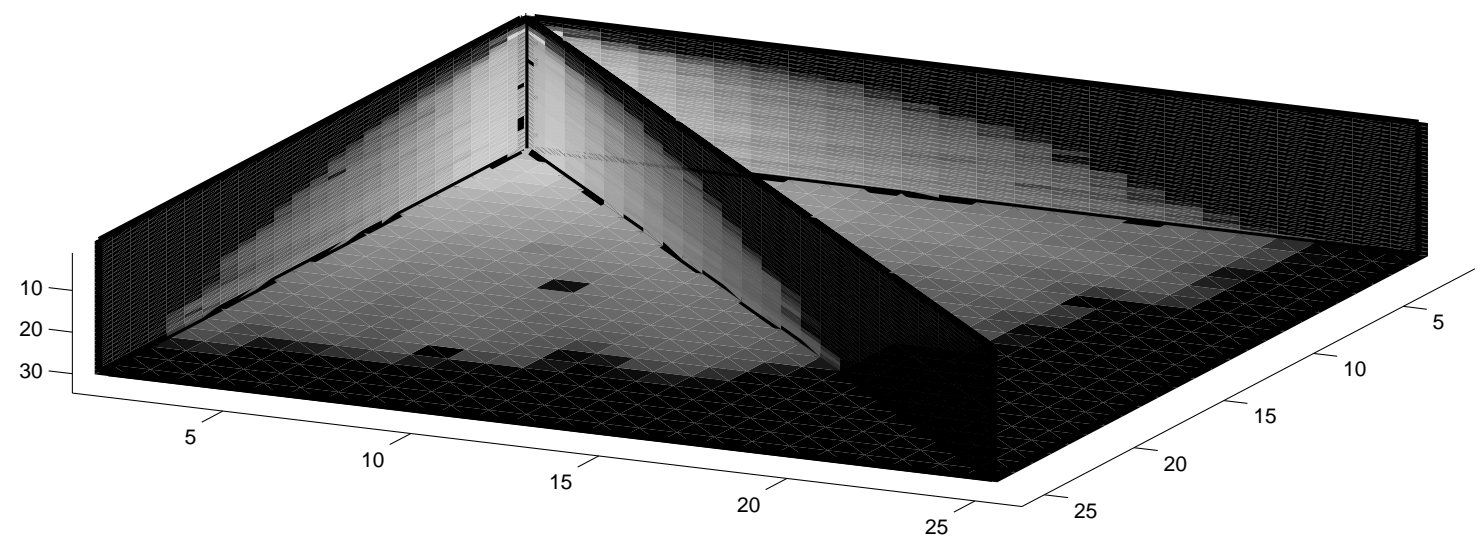

(c) Upscaling

Figure 4.20: Saturation maps for 0.15 PVI using the three methods (fine grid, nested gridding and upscaling) with an upscaling factor equal to $(3 \times 3 \times 3)$. The gravity number is set to $N_{g}=2$ 
Table 4.3: Speed-up factor and memory requirements of the nested gridding method for a pressure solve and for the whole simulation on the $3 D$ example presented in the chapter. The numbers in parenthesis in the third column are speed-up factors for the case where $N_{g}=2$

\begin{tabular}{cccccc}
\hline & Upscaling factor & \multicolumn{2}{c}{ Speed-up factor } & \multicolumn{2}{c}{ Memory } \\
& & $P$ solve & sim & RAM & $\%$ saved \\
\hline \multirow{3}{*}{ RES3 } & $3 \times 3 \times 1$ & 14 & $5.8(3)$ & 200 & 20 \\
\cline { 2 - 6 } & $3 \times 3 \times 3$ & 37 & $8.5(4)$ & 164 & 35 \\
\cline { 2 - 6 } & $9 \times 9 \times 3$ & 17 & $6.2(3)$ & 146 & 42 \\
\cline { 2 - 6 } & $9 \times 9 \times 9$ & 2.2 & 2.1 & 150 & 40 \\
\hline
\end{tabular}

\subsection{Discussion and Conclusions}

We have proposed an approximate method, using nested gridding, to estimate recovery directly from a fine grid. We showed some simple examples that demonstrated that the method was more accurate than using coarse grid simulation with upscaled permeabilities, while being faster than direct fine grid simulation.

The method used streamline-based simulation to move the saturations on the fine grid. For large $3 D$ problems it has been shown previously that streamline-based simulation is up to 100 times faster than conventional finite difference methods [10]. Our nested gridding method is almost up to another factor of 8.5 faster for large problems giving an overall speed-up up to 850 while retaining reasonable accuracy.

While the nested gridding method has been shown to give accurate results for the cases studied, the speed-up compared with direct fine-scale simulation is modest. This is because the transport of fluid along streamline on the fine grid dominates the overall CPU time for large $3 D$ problems. Our streamline code has not been optimized, however. Furthermore the movement of fluid along streamlines is easy to implement in parallel, offering potentially substantial time savings.

Many upscaling techniques are applicable only to a given set of boundary conditions (well rate or locations) [31]. The nested gridding method, since it retains an explicit representation of the saturation distribution on the fine grid, automatically accommodates changing well rates and location. 


\title{
5. Analytical Calculation of Minimum Miscibility Pressure for Injection Gases with One or Two Components
}

\author{
Yun Wang and Franklin M. Orr, Jr.
}

Analysis of the problem of one-dimensional, dispersion-free displacement of a multicomponent oil by a gas such as $\mathrm{CO}_{2}$, or methane has shown that the behavior of the flow is controlled by a sequence of key tie lines. Those that extend through the original oil and injected gas compositions and $n_{c}-3$ tie lines known as crossover tie lines. The minimum miscibility pressure (MMP) is the lowest pressure at which any of the key tie lines is a critical tie line. We show how to identify the $\mathrm{n}_{\mathrm{c}}-3$ crossover tie lines for oils that contain an arbitrary number of components as a sequence of tie lines whose extensions intersect. For displacement by a gas that contains only one component, that problem is equivalent to the problem of performing a flash calculation for single phase mixture with the composition of the intersection point. We transform the standard flash equation to a form that gives convergent solutions even for compositions well outside the phase diagram. We use the modified flash calculation to determine which of the key tie lines approaches the critical locus as the pressure is increased. Calculation of tie line lengths for a sequence of increasing pressures to determine the MMP as the lowest pressure at which the length of one of the key tie-lines becomes zero. Extension of the method to systems with two components in the injection gas is demonstrated for a four-component system, and further extension to multicomponent injection gases is discussed.

\subsection{Introduction}

Gas injection processes are among the most effective methods of enhanced oil recovery. A key parameter in the designing of a gas injection project is the minimum miscibility pressure (MMP), the pressure at which the local displacement efficiency approaches $100 \%$. If the flow is one-dimensional (1D) and there is no dispersive mixing, then the displacement efficiency is exactly $100 \%$ at the MMP. In real displacements, however, those conditions are not strictly satisfied. At pressures near MMP, the phases that form in the transition zone between injected fluid and original oil in place have very low interfacial tension, and the displacement is close to piston-like even when small amounts of dispersion or nonuniform flow due to viscous fingering or gravity segregation are present. That is the situation that arises in slim tube displacements, for example, displacement experiments that are routinely used to determine the MMP. In those experiments a long (say 10 $\mathrm{m}$ or longer), small diameter (say $0.5 \mathrm{~cm}$ ) tube packed with sand or glass beads is filled with oil that is then displaced by injection gas at a fixed temperature and outlet pressure. The fraction of the original oil in place recovered after injection of some fixed amount of gas (usually 1.1 or 1.2 pore volumes) is measured. The fraction of oil recovered is then plotted as a function of pressure. Typically, recovery increases rapidly with increasing pressure and then levels off. The MMP is usually taken to be the intersection of lines drawn through recovery points in the steeply climbing and level regions as long as the recovery in the level region is above some arbitrary cutoff (often $90 \%)$.

Most attempts to predict MMPs have been empirical. Investigators have tried to fit experimental MMPs to a variety of expressions for specific gas systems (see, for example, Holm and

\footnotetext{
${ }^{4}$ The material in this chapter was published as "Analytical Calculation of Minimum Miscibility Pressure," Fluid Phase Equilibria (1997) 139, 101-124.
} 
Josendal [96]; Glaso [73]; Orr and Silva [163]). While those approaches reproduce reasonably well the experimental observations on which they were based, they should be used with caution for injection gases or oils with compositions substantially different from those used to build the correlation (Orr and Silva [163]).

Computational attempts to calculate the MMP include three approaches: One-dimensional compositional simulations, mixing cell calculations based on a single cell, and multiple mixing cell calculations. Compositional simulations can be used to determine the MMP provided that sufficiently fine grids are used in the computations, the prediction of the MMP will be as accurate as the representation of the phase behavior by the equation of state used. The disadvantage of the simulation approach is the lengthy computation time for fine-grid simulations performed at many pressures.

Other investigators have used mixing cell methods (Pedersen et al. [175]; Jensen and Michelsen [102]; Neau et al. [148]) to estimate MMPs. In those methods, injected gas is first mixed with oil and flashed. The resulting vapor is mixed with fresh oil (forward contacts) and the liquid is mixed with fresh injection gas (reverse contacts), and the new mixtures are flashed. The process is repeated until it converges. The forward contacts converge to the tie line that extends through the oil composition (if there is one), and the reverse contacts converge to the tie line through the injection gas composition. The pressure is then increased until one of the tie lines is a critical tie line, which is taken to be the MMP. These methods work well as long as it is oil tie line (vaporiaing gas drive) or the injection gas tie line (condensing gas drive) that controls miscibility. In many systems, however, the MMP occurs at a pressure at which neither the oil tie line nor the gas tie line is a critical tie line, though such behavior can only occur if four or more components are present (Orr et al. [164]). The condensing/vaporizing drive described by Zick [230] and Stalkup [198] is an example of this behavior (Johns et al. [109]). For those systems, mixing cell methods do not give accurate predictions.

Multicell versions of the mixing cell idea have also been used. Metcalfe et al. [138] described a technique in which gas is added to the first cell and the contacts are flashed. The resulting gas phase is then moved to the next cell while more gas is added to the initial cell, and both cells are flashed. Again gas is moved to downstream cells. Thus, the procedure is similar to one-dimensional simulation, though fluids are not moved according to relative permeability functions. Hearn and Whitson [83] cite unpublished work by Zick that shows that multicell methods can be used for condensing-vaporizing drives.

In this report, we attempt to avoid the inaccuracies that can arise from finite grids or numbers of mixing cells, as well as the computational effort of many flash calculations in many cells, by calculating the MMP directly from analytical solutions for multicomponent, two-phase flow. We define the MMP based on the assumption of 1D, dispersion-free flow. Under those assumptions, the MMP can be calculated rigorously from analytical solutions for one-dimensional flow obtained by the method of characteristics (MOC). We begin with a brief review of the MOC theory to show that the problem of finding the MMP can be reduced in many cases to the problem of finding a sequence of tie lines whose extensions intersect at compositions that may lie well outside the region of physically attainable compositions. Next we formulate and solve a negative flash algorithm that converges for the compositions of the intersection points and use it to determine the sequence of tie lines. We then adjust the displacement pressure until one of the key tie lines becomes a critical tie line, one that is tangent to the critical locus (Johns and Orr [108]). We describe a technique that can be used to find the MMP for a two-component injection gas, and we discuss the extension of the technique to systems with more components. 


\subsection{Mathematical Model}

For one-dimensional, dispersion-free two-phase flow in which components do not change volume as they transfer between two phases, the mass conservation equations are

$$
\frac{\partial C_{i}}{\partial t}+\frac{\partial F_{i}}{\partial z}=0, \quad i=1, \ldots, n_{c}
$$

where $t$ and $z$ are dimensionless time and distance, respectively. Components do change volume as they transfer between phases, but that volume change does not affect the MMP (Dindoruk [43]). $C_{i}$ is the overall volumetric fraction of component $i$ given by:

$$
C_{i}=c_{i l}\left[1+\left(K_{i}-1\right) S\right], \quad i=1, \ldots, n_{c},
$$

$F_{i}$ is the the overall fractional flux of component $i$ given by:

$$
F_{i}=c_{i l}\left[1+\left(K_{i}-1\right) f\right], \quad i=1, \ldots, n_{c},
$$

Here $c_{i l}$ is the volume fraction of component $i$ in the liquid phase; $S$ is gas saturation; $f$ is the fractional flow of the gas phase; and $K_{i}$ is the equilibrium ratio of component $i$.

For the calculations reported here, the fractional flow function was assumed to be

$$
f=\frac{S^{2}}{S^{2}+(1-S)^{2}}
$$

It should be noted that while the fractional flow function is required to construct analytical solutions to Eq. 5.1, numerical calculations suggest that it has no effect on the key tie line geometry that determines the MMP. Two additional constraints associated with Eq. 5.1 are:

$$
\sum_{i=1}^{n_{c}} C_{i}=1
$$

and

$$
\sum_{i=1}^{n_{c}} F_{i}=1,
$$

which means that only $n_{c}-1$ of the conservation equations are independent.

Eqs. 5.1-5.6 can be solved by calculating the velocity at which a given overall composition propagates. An eigenvalue problem results, in which an eigenvalue is the wave speed at which the composition moves. The associated eigenvector gives a discrete direction in composition space along which composition variations satisfy the differential equations. Integration along eigenvector directions gives many 'paths' through the $n_{c}$ dimensional composition space. For example, Fig. 5.1 shows typical composition paths for a four-component system in which equilibrium K-values are independent of composition (Note that when equilibrium K-values are independent of compositions the surfaces of liquid and vapor compositions are both planes. In Fig. 5.1, the surface of liquid compositions is the triangle $\mathrm{ABC}$, and the surface of vapor compositions is $\mathrm{DEF}$ ). Tie lines are paths, and there are two nontie-line paths through each composition point. The solution to the problem is the unique route that connects the initial oil composition to the injection gas composition (Monroe et al. [143]). The modern form of the theory was developed for analysis of three-component surfactant flooding (Larson and Hirasaki [126]; Larson [125]; Helffreich [85]; Hirasaki [93]), though mathematically equivalent approaches were reported earlier by Welge et al. [223] and Wachman [218] for gas/oil and alcohol displacement systems. Application of the theory to gas/oil systems continued with the analysis of three-component systems by Dumore et al. [50]. Monroe et al. [143] 


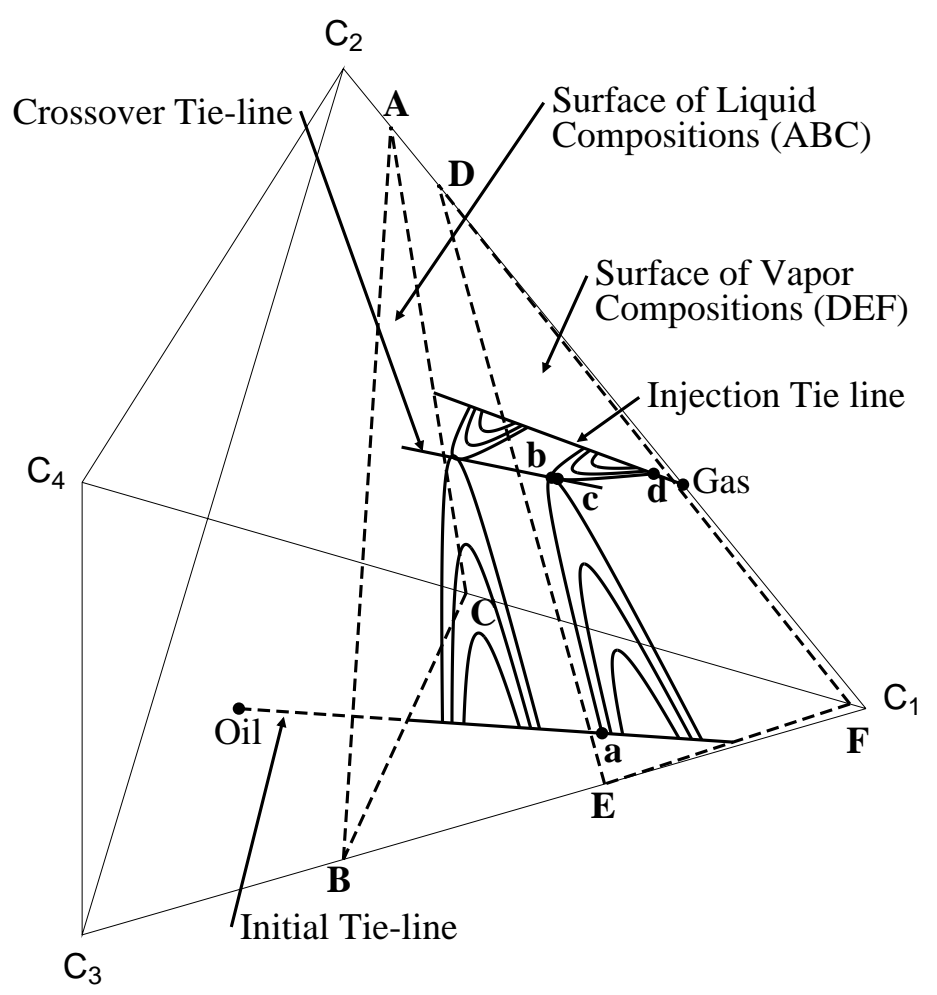

Figure 5.1: Composition paths in a four-component system with constant K-values (Points a, b, c, $\mathbf{d}$ refer to composition points in Fig. 5.2.

obtained the first solutions for systems with four components, and subsequent work by Johns [107] and Dindoruk [43] explored the behavior of systems with four or more components (Dindoruk [43]; Orr et al. [164]; Johns et al. [109]; Johansen et al. [110]). Those papers showed that the solution behavior is controlled by $n_{c}-1$ key equilibrium tie lines. They are the tie lines that extend through injection gas and initial oil compositions as well as $n_{c}-3$ additional tie-lines known as 'crossover' tie lines (Monroe et al. [143]). If the pressure or enrichment is increased enough that one of the key tie lines becomes a critical tie line (a tie line of zero length that is tangent to the critical locus), then the displacement is multicontact miscible (Orr et al. [164]; Johns et al. [109]). Thus the first step toward finding the MMP is to identify the key tie lines.

In portions of the solution where compositions vary continuously, the variation must occur along paths. It often happens, however, that eigenvalues vary along a path in such a way that fast-moving compositions would appear upstream of slow-moving ones, a physical impossibility. In such situations, the continuous variation (known as a rarefaction by analogy with gas dynamics) is replaced by a shock, which must satisfy a material balance (also known as a Rankine-Hugoniot condition) of the form

$$
\Lambda=\frac{F_{i}^{U}-F_{i}^{D}}{C_{i}^{U}-C_{i}^{D}}, \quad i=1, \ldots, n_{c},
$$

where $\Lambda$ is the shock velocity, superscripts $U$ and $D$ represent the upstream and downstream sides of the shock. A shock must also occur whenever the number of phases present changes; the solution route must enter and exit the two-phase region via a shock along a tie line extension (Helfferich [85]).

Fig. 5.2 shows an example, again for the constant K-values, in which a shock between two tie lines would be required. It shows the magnitudes of composition velocities, $\lambda_{n t}$, along the path 


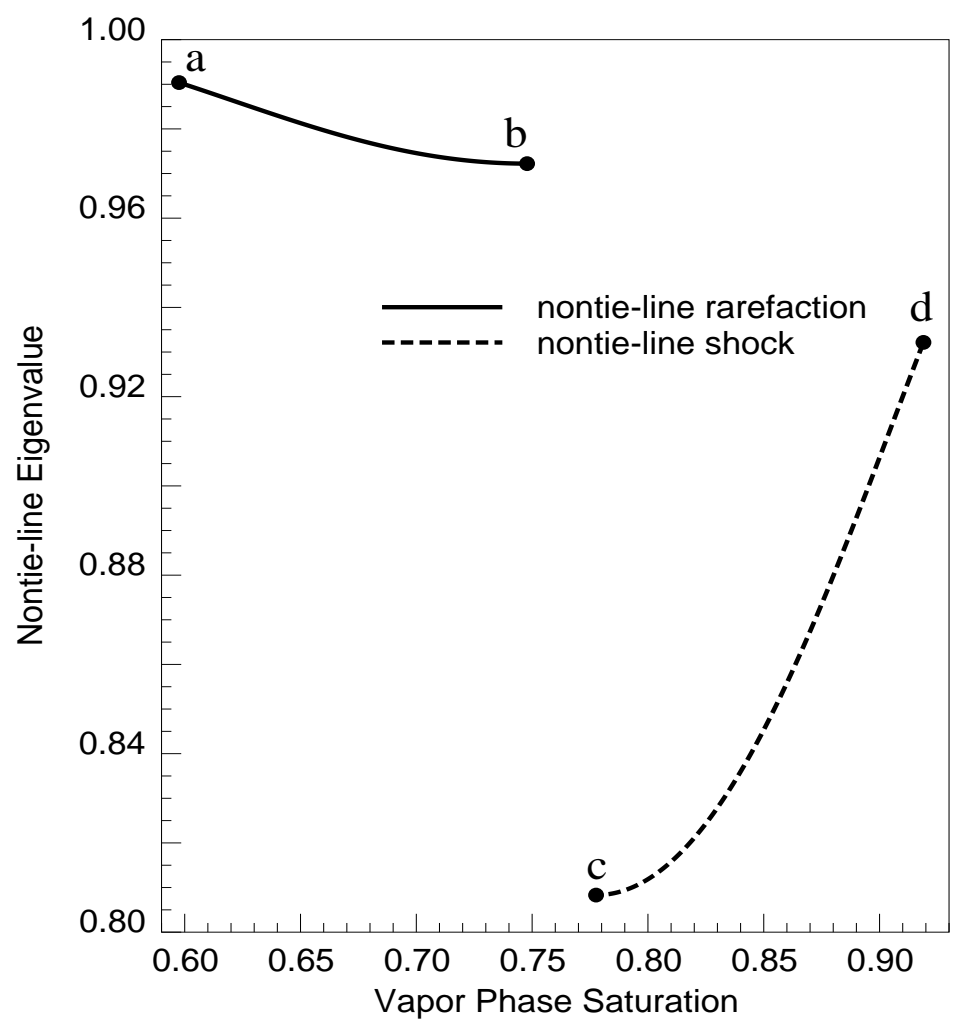

Figure 5.2: Variation of the nontie-line eigenvalue alonf the nontie-line path.

traced from $a$ to $b$ in the 'vertical' surface of paths and then from $c$ to $d$ in the 'horizontal' surface of paths in Fig.1 (between $b$ and $c$ there is a short variation along the crossover tie line). Value of $\lambda_{n t}$ decreases as the path is traced toward the direction from $a$ to $b$, so a rarefaction is permitted there. From $c$ to $d$, however, the value of $\lambda_{n t}$ increases, which would violate the velocity constraints if this path were being traced in the upstream direction. Hence, this composition variation would be 'self-sharpening', and a shock would connect the tie lines that contain points $c$ and $d$. An important result of the analytical theory is that the extensions of two tie lines connected by a shock must intersect (Johns [107]; Orr et al. [164]; Johns et al. [109]).

For systems in which all the tie lines are connected by shocks, which we refer to as fully self-sharpening, all the crossover tie lines can be determined from the requirement that the tie line extensions intersect. Fig. 5.3 shows such a system. In Fig. 5.3, the initial oil composition is $o$, a mixture of methane $\left(\mathrm{CH}_{4}\right)$, butane $\left(\mathrm{C}_{4}\right)$ and decane $\left(\mathrm{C}_{10}\right)$. The curves labeled 'phase envelopes' in Fig. 5.3, are the binodal curves of liquid and vapor compositions in the four ternary faces that bound the quaternary diagram. The injection gas is a mixture of $\mathrm{CO}_{2}$ and $\mathrm{CH}_{4}$ (composition $g$ ). The initial tie line lies in the $\mathrm{CH}_{4} / \mathrm{C}_{4} / \mathrm{C}_{10}$ ternary face, and the injection gas tie line is on the $\mathrm{CH}_{4} / \mathrm{CO}_{2} / \mathrm{C}_{10}$ face. The extensions of the crossover tie line intersect both the initial oil and injection gas tie lines. In this system, if the pressure were increased, the crossover tie line would become tangent to the critical locus at a pressure lower than that of either the initial oil or injection gas tie lines.

For ternary systems, self-sharpening behavior can be identified easily from an analysis of systems with constant K-values (see Appendix A of Johns [107] or Johns et al. [109] for details). The analysis shows that self-sharpening behavior is controlled by whether the value of the intermediate $\mathrm{K}$-value is greater than or less than one. While that analysis is only approximate for systems with composition-dependent K-values, the prediction of self-sharpening behavior is quite reliable. For systems with more than three components, self-sharpening behavior can always be detected by 


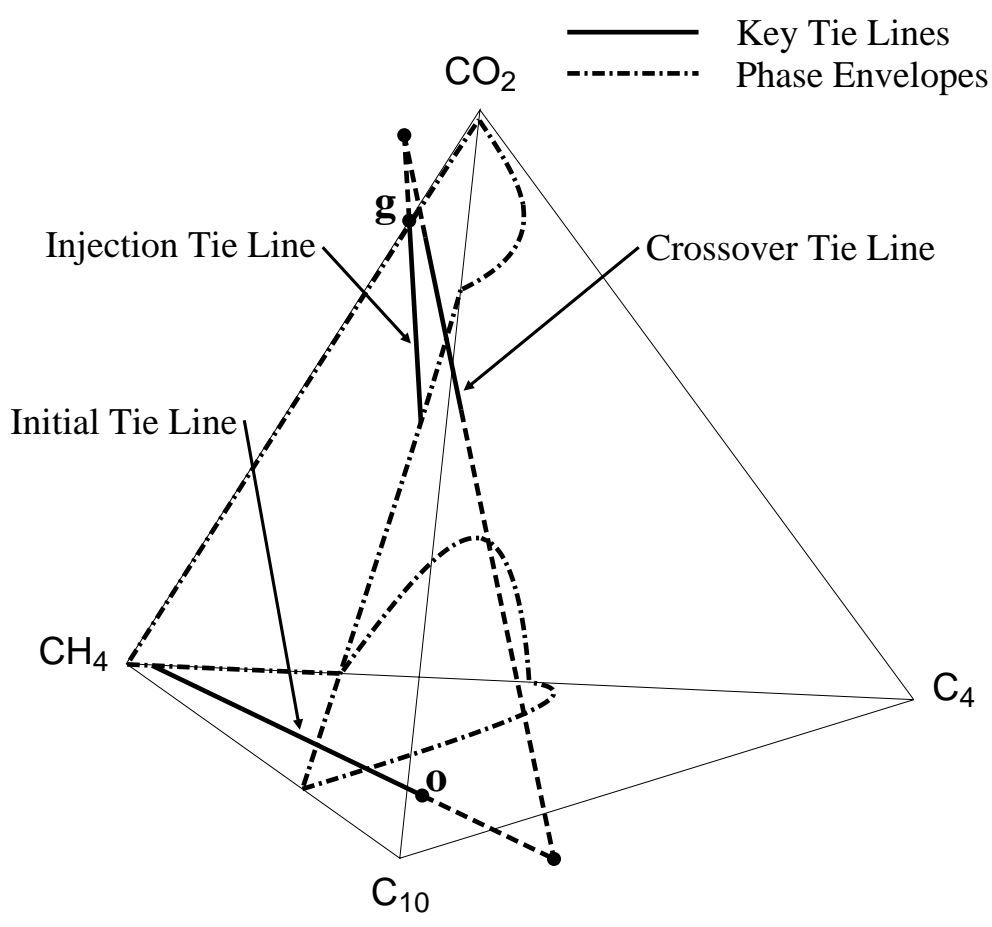

Figure 5.3: Key tie lines for a displacement of a three-component oil (composition o) by a twocomponent gas (composition $\mathrm{g}$ ) at $160^{\circ} \mathrm{F}\left(71.1^{\circ} \mathrm{C}\right), 1600 \mathrm{psia}(11.0 \mathrm{MPa})$. The crossover tie line intersects extensions of the initial oil and injection gas tie lines. See Table 5.3 for oil and gas compositions. 
direct integration along nontie-line paths. An approximate method for identifying self-sharpening behavior in multicomponent displacements was outlined by Johns and Orr [108], who reported a procedure for calculation of the MMP for displacement of a multicomponent oil by a singlecomponent gas. In that scheme, components present at any point in a displacement are lumped into a pseudoternary system so that the expression given by Johns and Orr [108] could be used to determine self-sharpening behavior.

In the remainder of this report we develop a technique for calculation of MMP that is rigorous for systems that are fully self-sharpening. Experience with solutions for $\mathrm{CO}_{2}$ displacements, for example, indicates that the fully self-sharpening systems are common for $\mathrm{CO}_{2}$ injection. We also have computational evidence that this approach gives accurate MMPs for displacements that include one or more segments of rarefaction along nontie-line paths, but we do not have a rigorous proof of accuracy for those systems.

We find the key tie-lines by solving a sequence of flash calculations for the compositions at intersection points of tie lines. Those points always lie in the single-phase region, and they often fall outside the phase diagram where some component fractions are negative (see Fig. 5.3 for an example). For single-phase compositions that are not too far from the two-phase region, the negative flash of Whitson and Michelsen [225] can be used to determine the tie-line that extends through that composition. However, many of the intersection points that arise in the analytical solutions are well outside the domain of convergence of the negative flash algorithm of Whitson and Michelsen. In the next section, we transform the flash calculation and develop a negative flash algorithm that does converge for such compositions.

\subsection{Modified Negative Flash}

The equation of any tie line is given by a material balance of the form

$$
C_{i}=c_{i l}(1-S)+c_{i v} S, i=1, \ldots, n_{c},
$$

which can be rearranged to yield Eq. 5.2 by substitution of the definition of the equilibrium K-values,

$$
K_{i}=\frac{c_{i v}}{c_{i l}}, i=1, \ldots, n_{c}
$$

where $C_{i}$ is the overall composition for component $i, c_{i l}$ is the volume fraction of component $i$ in the liquid phase and $c_{i v}$ is the volume fraction in the vapor phase. $S$ is the vapor phase saturation. $K_{i}$ is the equilibrium ratio for component $i$ (K-value). The equilibrium compositions must also sum to unity,

$$
\sum_{i=1}^{n_{c}} c_{i l}=\sum_{i=1}^{n_{c}} c_{i v}=1 .
$$

The usual approach to a flash calculation follows that of Rachford and Rice [181]. For a given set of K-values, the following equation is solved iteratively for $S$,

$$
F(S)=\sum_{i=1}^{n_{c}} \frac{C_{i}\left(K_{i}-1\right)}{1+\left(K_{i}-1\right) S}=0 .
$$

The phase compositions are then calculated as 


$$
\left\{\begin{aligned}
c_{i l} & =\frac{C_{i}}{1+\left(K_{i}-1\right) S} \\
c_{i v} & =\frac{K_{i} C_{i}}{1+\left(K_{i}-1\right) S}
\end{aligned}\right.
$$

Fugacities can be calculated from an equation of state (EOS). Here we use the PengRobinson EOS [177] to calculate component partial fugacities. K-values are then updated using fugacities until convergence is reached. Phase compositions in mole fractions are converted to volume fractions using the pure component molar densities.

Rachford and Rice assumed that the overall composition is inside the two-phase region. Whitson and Michelsen [225] pointed out that single-phase compositions can be flashed with the same algorithm if the vapor phase saturation is not restricted to lie between zero and one. They showed that their negative flash always converges for compositions within a range bounded by $1 /\left(1-K_{\max }\right)$ and $1 /\left(1-K_{\min }\right)$, where $K_{\max }$ and $K_{\min }$ are the highest and lowest K-values of the system.

We observe that, for an arbitrary overall composition, the vapor phase saturation $S$ can vary in an unrestricted way, while the phase compositions $c_{i l}$ and $c_{i v}$ are always subject to the restriction that they be greater than zero and less than one. Hence we choose phase compositions instead of vapor phase saturation as the primary variables. Elimination of $S$ yields the following system of equations:

$$
\left\{\begin{array}{l}
\sum_{i=1}^{n_{c}} c_{i l}-1=0 \\
C_{i} c_{1 l}\left(K_{1}-1\right)-c_{1 l} c_{i l}\left(K_{1}-K_{i}\right)-c_{i l} C_{1}\left(K_{i}-1\right)=0, \quad i=2, . ., N_{c}
\end{array}\right.
$$

Eq. 5.13 can be solved for the liquid phase compositions by a Newton-Raphson iteration of the form

$$
\mathbf{J} \Delta \mathbf{c}_{1}=-\mathbf{F},
$$

where $\Delta c_{l}$ is the vector of changes in individual component fractions. The entries in $\mathrm{F}$ are

$$
\begin{gathered}
F_{1}=\sum_{i=1}^{n_{c}} c_{i l}-1 \\
F_{i}=C_{i} c_{1 l}\left(K_{1}-1\right)-c_{1 l} c_{i l}\left(K_{1}-K_{i}\right)-c_{i l} C_{1}\left(K_{i}-1\right) \quad i=2, . ., n_{c} .
\end{gathered}
$$

The Jacobian matrix $\mathbf{J}$, whose elements are derivatives of $\mathbf{F}$ with respect to $c_{i l}$, is

$$
\mathbf{J}=\left[\begin{array}{ccccc}
1 & 1 & 1 & \ldots & 1 \\
J_{21} & J_{22} & 0 & \ldots & 0 \\
J_{31} & 0 & J_{33} & \ldots & 0 \\
\cdot & \cdot & \cdot & \cdot & \cdot \\
J_{n_{c} 1} & 0 & 0 & \ldots & J_{n_{c} n_{c}}
\end{array}\right]
$$


where

$$
\left\{\begin{array}{l}
J_{i 1}=C_{i}\left(K_{1}-1\right)-c_{i l}\left(K_{1}-K_{i}\right) \\
J_{i i}=-c_{1 l}\left(K_{1}-K_{i}\right)-C_{1}\left(K_{i}-1\right) \quad i=2, . ., n_{c}
\end{array}\right.
$$

The simple structure of the Jacobian matrix yields a straightforward algebraic solution of the form

$$
\left\{\begin{array}{c}
\Delta c_{1 l}=\frac{\sum_{i=2}^{n_{c}} \frac{F_{i}}{J_{i i}}-F_{1}}{1-\sum_{i=2}^{n_{c}} \frac{J_{i 1}}{J_{i i}}} \\
\Delta c_{i l}=-\frac{F_{i}+J_{i 1} \Delta c_{1 l}}{J_{i i}} \quad i=2, . ., n_{c}
\end{array}\right.
$$

The updated phase compositions are

$$
c_{i l}^{\text {new }}=c_{i l}^{\text {old }}+\Delta c_{i l} \quad i=1, . ., n_{c} .
$$

The iterative process ends when the left hand side of Eq. 5.13 is zero to within some tolerance. Then the phase compositions are used to calculate new partial fugacities and the Kvalues are updated. Here we use the standard successive substitution

$$
K_{i}^{\text {new }}=K_{i}^{\text {old }}\left(\frac{f_{i}^{L}}{f_{i}^{V}}\right) \quad i=1, . ., n_{c},
$$

where $f_{i}^{L}$ and $f_{i}^{V}$ are the liquid and vapor partial fugacities for component i, respectively, and the K-values in Eq. 5.20 are defined in terms of mole fractions. The flash calculation is complete when component partial fugacities are equal (to within some tolerance). We note that there is an opportunity to improve the speed of the calculations performed be acceleration along the lines described by Michelsen [139]. For the examples reported in this report, however, the computation times for calculating MMPs were quite short (on the order of 3-10 minutes on a workstation), so the speed of the successive substitution was not a limiting factor.

Fig. 5.4 shows three overall compositions used to test the performance of the modified negative flash, and Fig. 5.5 compares convergence behavior of the modified flash with that of the negative flash of Whitson and Michelsen. Composition $\mathbf{A}$ is in the domain of convergence of the Whitson-Michelsen negative flash (The vapor phase saturation for point $\mathbf{A}$ is -0.19799, well within the domain of convergence $-1.79176<\mathrm{S}<1.01865)$. Both methods converge quickly. Compositions $\mathbf{B}$ and $\mathbf{C}$ are outside the domain of convergence for the Whitson-Michelsen negative flash, but Fig. 5.5 shows that the modified negative flash converges quickly for those compositions as well. Points $\mathbf{B}$ and $\mathbf{C}$ are actually outside the phase diagram; the mole fraction of $\mathrm{CO}_{2}$ is negative. They

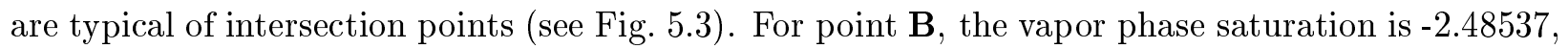
which is clearly outside the convergence domain $-2.14178<\mathrm{S}<1.03103$. Composition $\mathrm{C}$ lies on the extension of a tie line that is quite close to the critical point. The vapor phase saturation of point $\mathbf{C}$ is -3902.968 , which is also outside the domain of convergence defined by the largest and smallest K-values: $-3443.336<\mathrm{S}<323.810\left(K_{\max }=1.000290, K_{\min }=0.996912\right)$. In this case as well, the modified negative flash converges rapidly. It is important that the flash algorithm perform well in the near-critical region if it is to be used to calculate the MMP. 


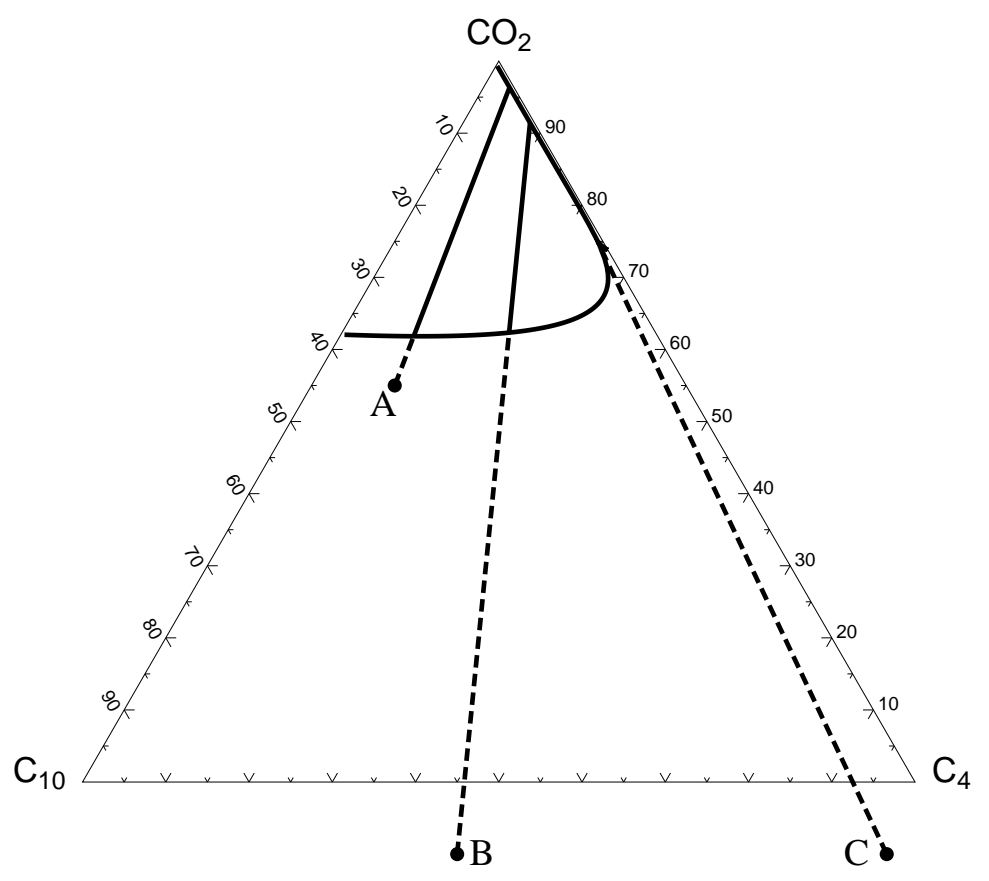

Figure 5.4: Composition for test of the modified negative flash algorithm $\left(\mathrm{T}=160^{\circ} \mathrm{F}\left(71.1^{\circ} \mathrm{C}\right)\right.$, $\mathrm{P}=1600$ psia $(11.0 \mathrm{MPa}))$.
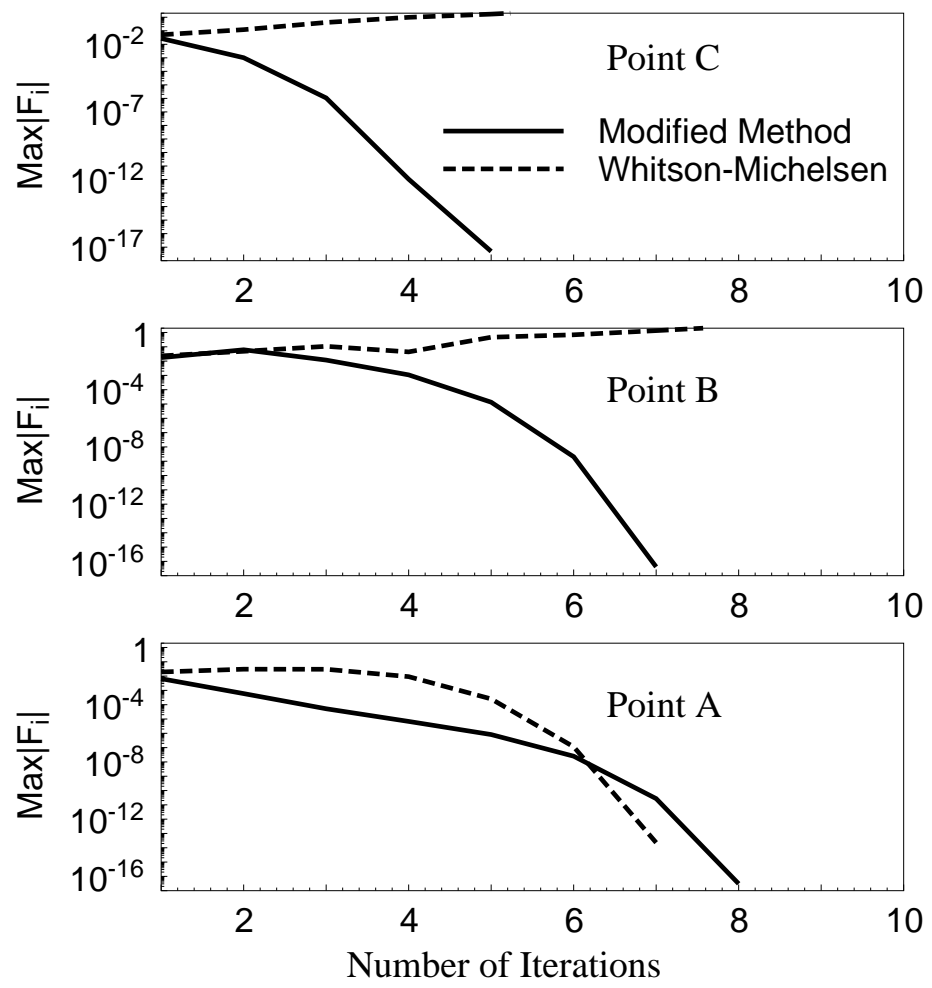

Figure 5.5: Convergence of the modified negative flash. 
Table 5.1: Oil and gas compositions for pure $\mathrm{CO}_{2}$ injection, $\mathrm{T}=160^{\circ} \mathrm{F}$.

\begin{tabular}{|c||c|c|c|c|c|c|}
\hline \hline \multirow{2}{*||}{ Component } & \multicolumn{5}{|c|}{ Compositions(Mole Fraction) } \\
\cline { 2 - 7 } & $\mathrm{CH}_{4}$ & $\mathrm{CO}_{2}$ & $\mathrm{C}_{4}$ & $\mathrm{C}_{10}$ & $\mathrm{C}_{14}$ & $\mathrm{C}_{20}$ \\
\hline Oil & 0.20 & 0.05 & 0.05 & 0.40 & 0.10 & 0.20 \\
\hline Gas & 0.00 & 1.00 & 0.00 & 0.00 & 0.00 & 0.00 \\
\hline
\end{tabular}

\subsection{Analytical Calculation of MMP}

\subsubsection{Pure $\mathrm{CO}_{2}$ Injection}

To illustrate how the modified negative flash can be used to determine the MMP, we consider first an example in which pure $\mathrm{CO}_{2}$ displaces a six-component oil. This system is fully self-sharpening: all the nontie-line portions of the solution are shocks. Hence, all the adjacent key tie lines intersect. We now show that the intersection points are such that exactly one component changes to zero composition across each shock that connects two tie lines. Foe the calculations reported, component properties were those reported by Johns [107].

Table 5.1 shows the oil and gas compositions and system temperature. The initial and injection tie lines are both known. The initial tie line includes all six components that are present in the oil. The injection tie line contains two components, $\mathrm{CO}_{2}$ and $\mathrm{C}_{20}$. There are three crossover tie-lines altogether for a six-component system.

We show now that one component disappears across each shock between tie lines in the sequence from the initial tie line to the injection tie line. Assume first that there are two intersecting tie lines that have non-zero fractions of an identical number of components. Now the number of components present can only change if the tie line changes. Because there are only three crossover tie lines, there must be another pair of adjacent intersecting tie lines that have two or more components missing. Suppose that components $\mathrm{j}$ and $\mathrm{k}$ are missing on tie-line $n$, then

$$
\begin{aligned}
& C_{j}=c_{j l}^{n}\left[1+\left(K_{j}^{n}-1\right) S_{n}\right]=0, \\
& C_{k}=c_{k l}^{n}\left[1+\left(K_{k}^{n}-1\right) S_{n}\right]=0,
\end{aligned}
$$

which requires that

$$
S_{n}=\frac{1}{1-K_{j}^{n}}=\frac{1}{1-K_{k}^{n}}
$$

Eq. 5.24 is satisfied only if $K_{j}^{n}=K_{k}^{n}$, an equality that never holds true except at the critical point as long as $\mathrm{K}$-values stay ordered. That condition is satisfied for this system and for systems of practical interest; if two components have almost equal K-values they should be lumped for compositional purposes. Thus, the assumption that two tie lines in the sequence have the same number of components leads to a contradiction. Hence we have shown that, for this system, there are four shocks that connect pairs of tie lines, and one component present in the initial oil disappears across each shock. The argument given applies to initial compositions with an arbitrary number of components. Therefore the statement applies for displacement of an oil containing any number of components by a pure component gas.

We use the disappearance of one component at each shock to locate easily the sequence of tie line intersection points. Components disappear in the order of their K-values: $\mathrm{CH}_{4}$ first in 
Table 5.2: Key tie-line structure for pure $\mathrm{CO}_{2}$ injection.

\begin{tabular}{|c||c|c|c|c|c|c|}
\hline \hline \multirow{2}{*}{ Tie-lines } & \multicolumn{7}{|c|}{ Compositions } \\
\hline & $\mathrm{CH}_{4}$ & $\mathrm{CO}_{2}$ & $\mathrm{C}_{4}$ & $\mathrm{C}_{10}$ & $\mathrm{C}_{14}$ & $\mathrm{C}_{20}$ \\
\hline Initial & $\sqrt{ }$ & & $\sqrt{ }$ & $\sqrt{ }$ & $\sqrt{ }$ & $\sqrt{ }$ \\
\hline Crossover 1 & & $\sqrt{ }$ & $\sqrt{ }$ & $\sqrt{ }$ & $\sqrt{ }$ & $\sqrt{ }$ \\
\hline Crossover 2 & & $\sqrt{ }$ & & $\sqrt{ }$ & $\sqrt{ }$ & $\sqrt{ }$ \\
\hline Crossover 3 & & $\sqrt{ }$ & & & $\sqrt{ }$ & $\sqrt{ }$ \\
\hline Injection & & $\sqrt{ }$ & & & & $\sqrt{ }$ \\
\hline
\end{tabular}

the shock from the initial tie line to crossover tie line $1 ; \mathrm{C}_{4}$ second in the shock from crossover tie line 1 to crossover tie line 2, and so on. Table 5.2 shows the sequence of tie lines for the example six-component oil displaced by $\mathrm{CO}_{2}$. For the disappearing component on the crossover tie line $n$,

$$
C_{j}=c_{j l}^{n}\left[1+\left(K_{j}^{n}-1\right) S^{n}\right]=0 .
$$

Therefore the vapor phase saturation on crossover tie line $n$ where the component $j$ is zero is

$$
S^{n}=\frac{1}{1-K_{j}^{n}}
$$

Substitution of Eq. 5.24 into Eq. 5.23 for other components gives the $i$ compositions of the intersection point,

$$
C_{i}=c_{i l}^{n}\left[\frac{K_{i}^{n}-K_{j}^{n}}{1-K_{j}^{n}}\right], \quad i \neq j .
$$

Application of the modified negative flash then gives the next tie line associated with the intersection point.

The lengths $l_{n}$ of the key tie-lines indicate how close they are to the critical region,

$$
l_{n}=\sqrt{\sum_{i=1}^{n_{c}}\left(c_{i v}^{n}-c_{i l}^{n}\right)^{2}}
$$

To find the MMP, we repeat the calculation of tie line lengths for increasing pressures. The MMP is the pressure at which one of the key tie lines reaches zero length, an indication that it is a critical tie line. Thus, for a pure component injection gas, the algorithm for calculation of the MMP is:

1. Perform a modified negative flash to find the initial oil and injection gas tie lines.

2. Find the intersection point between the initial tie line and crossover tie line 1 from Eq. 5.27 applied to each of the components in the oil,

$$
C_{i}^{1}=c_{i l}^{o}\left[\frac{K_{i}^{o}-K_{j}^{o}}{1-K_{j}^{o}}\right],
$$

where superscript $o$ denotes the initial tie line, and

$$
i=\mathrm{CH}_{4}, \mathrm{CO}_{2}, \mathrm{C}_{4}, \mathrm{C}_{10}, \mathrm{C}_{14}, \mathrm{C}_{20}, \quad j=\mathrm{CH}_{4} .
$$


3. Perform a modified negative flash to find crossover tie line 1.

4. Find the intersection point between crossover tie lines 1 and 2

$$
C_{i}^{2}=c_{i l}^{1}\left[\frac{K_{i}^{1}-K_{j}^{1}}{1-K_{j}^{1}}\right],
$$

where the superscript 1 denotes crossover tie line 1 , and

$$
i=\mathrm{CH}_{4}, \mathrm{CO}_{2}, \mathrm{C}_{4}, \mathrm{C}_{10}, \mathrm{C}_{14}, \mathrm{C}_{20}, \quad j=\mathrm{C}_{4} \text {. }
$$

5. Perform a modified negative flash to find crossover tie line 2.

6. Find the intersection point between crossover tie lines 2 and 3

$$
C_{i}^{3}=c_{i l}^{2}\left[\frac{K_{i}^{2}-K_{j}^{2}}{1-K_{j}^{2}}\right],
$$

where the superscript 2 denotes crossover tie line 2 , and

$$
i=\mathrm{CH}_{4}, \mathrm{CO}_{2}, \mathrm{C}_{4}, \mathrm{C}_{10}, \mathrm{C}_{14}, \mathrm{C}_{20}, \quad j=\mathrm{C}_{10} .
$$

7. Perform a modified negative flash to find the crossover tie line 3.

8. Increase system pressure and repeat the above steps until one of the key tie line lengths is zero.

Fig. 5.6 shows the change of key tie line lengths with pressure. As Fig. 5.6 indicates, the first crossover tie line reaches the critical region at lower pressure than any other tie line. Therefore it is the first crossover tie line that controls miscibility. Fig. 5.6 indicates that the MMP is 2380 psia (16.4 MPa). To confirm the MMP calculated analytically, we also carried out one-dimensional compositional simulations for the same system. We used a fully explicit finite difference simulator with single-point upstream weighting. The Peng-Robinson equation of state was used to calculate phase equilibria. The computations were performed with 800 grid blocks, and the Courant number $(C=\lambda \Delta t / \Delta x, \lambda$ is the eigenvalue) was set to 0.2. Fig. 5.7 shows results of those simulations reported as fractional recovery (defined as one minus the fraction of moles of hydrocarbons remaining within the porous medium) of hydrocarbon components at 1.1 pore volumes injected (the use of recovery at 1.1 pore volumes injected is arbitrary but customary in interpretations of slim tube displacements). We interpret the compositional simulation results as if they were experimental results: The estimated MMP is taken to be the intersection of lines drawn through recovery points in the steeply climbing and level regions. An estimated MMP of 2340 psia (16.1 MPa) is obtained by extrapolating the linear portions of the recovery curve to an intersection point. A more precise estimate of the MMP could have been obtained by applying the technique described by Zick [230] (extrapolation to an infinite number of grid blocks), though at the cost of some additional computation time. While the numerical results are clearly influenced by numerical dispersion (see Stalkup [198], Zick [230] or Walsh and Orr [219]), they are already accurate enough to confirm the analytical results.

Next we consider displacement of a ten-component oil by pure $\mathrm{CO}_{2}$. This oil was studied experimentally by Metcalfe and Yarborough [137], and Johns and Orr [108] reported an analytical solution for this oil. However, they encountered convergence difficulties in the near critical region when they attempted to calculate the MMP, and hence they obtained only an estimate. However, 


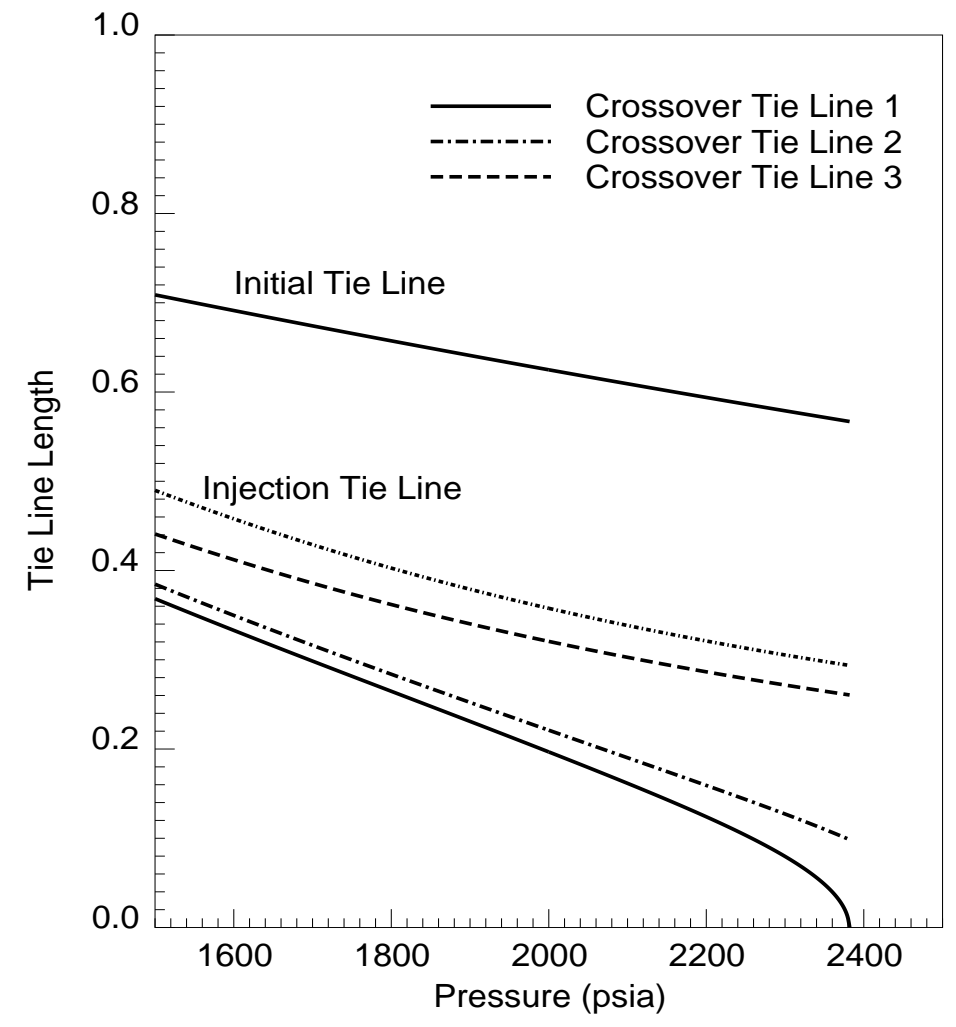

Figure 5.6: Dependence of key tie line lengths on pressure for displacement of a six-component oil by $\mathrm{CO}_{2}$ at $160^{\circ} \mathrm{F}\left(71.1^{\circ} \mathrm{C}\right)$. The MMP is 2380 psia $(16.4 \mathrm{MPa})$. See Table 5.1 for oil and gas compositions.

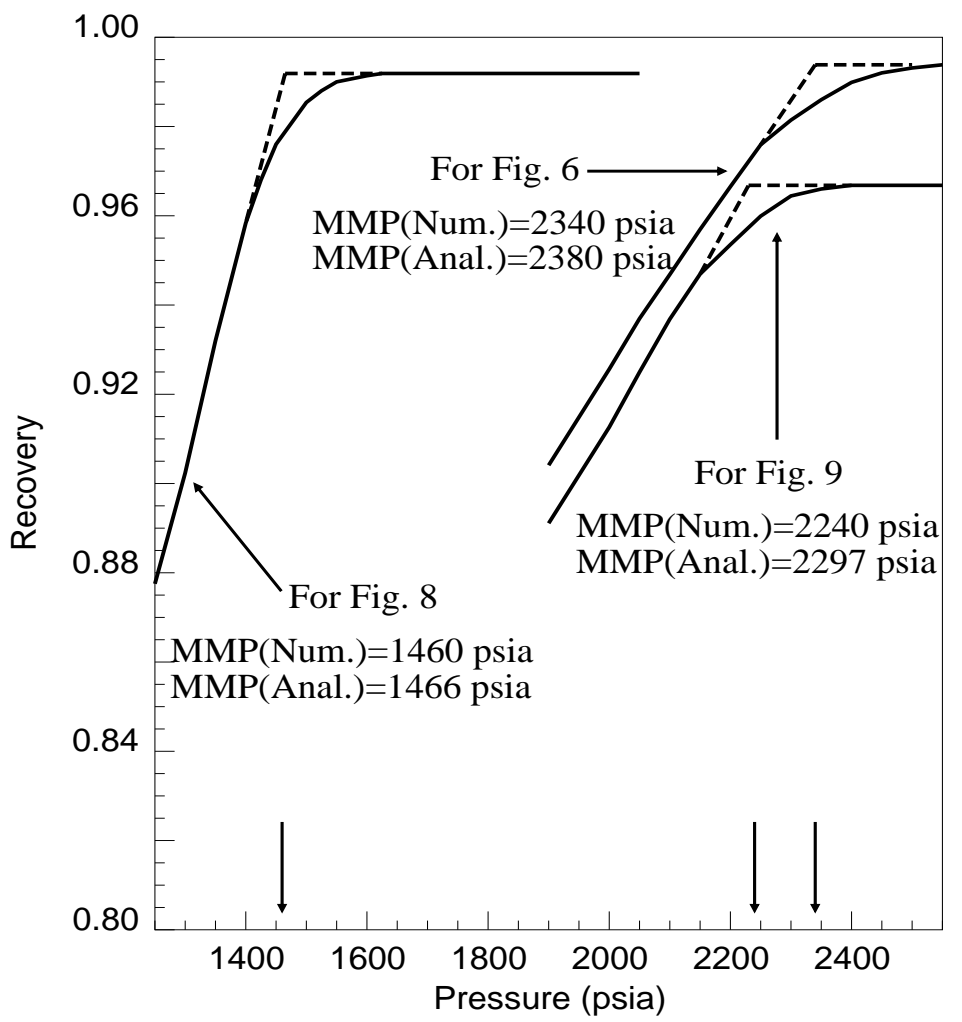

Figure 5.7: Results of one-dimensional compositional simulations with 800 grid blocks and a Courant number of 0.2, for the systems of Fig. 5.6, Fig. 5.8 and Fig. 5.9. 


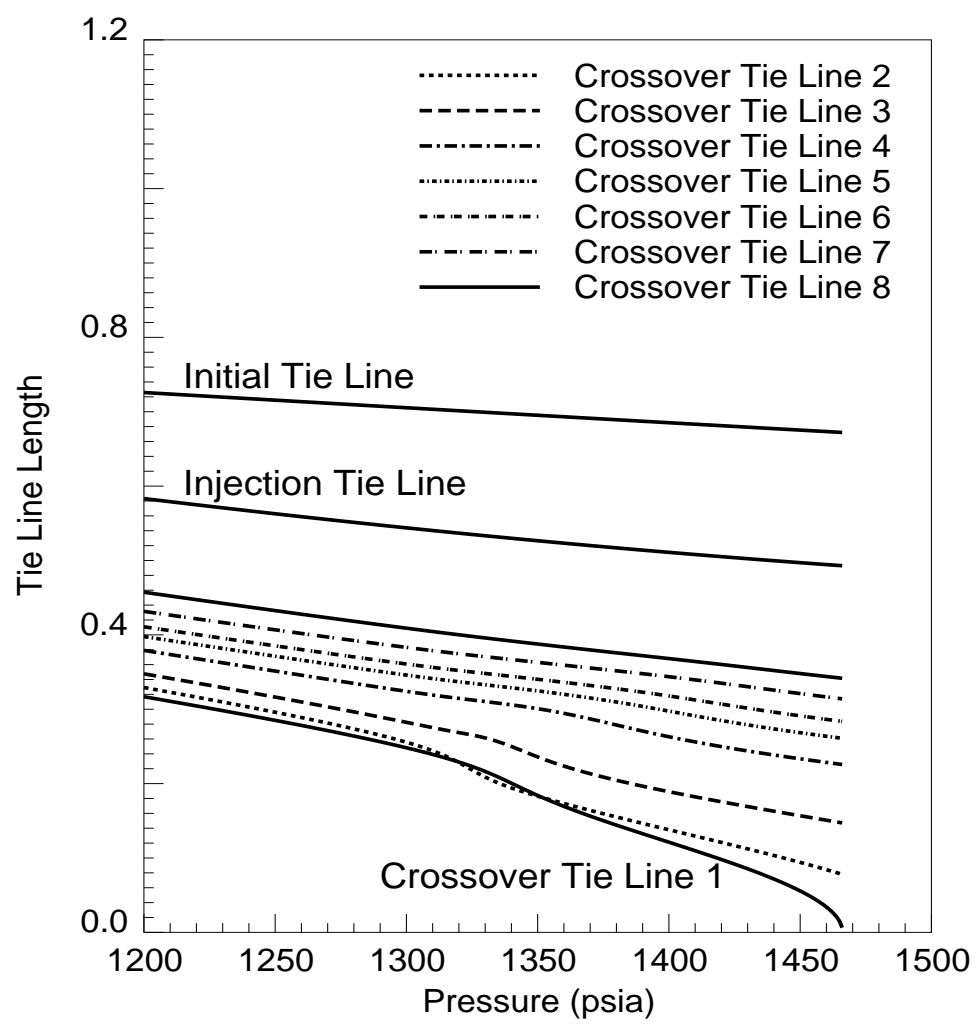

Figure 5.8: Key tie line lengths for $\mathrm{CO}_{2}$ displacement of a ten-component oil at $120^{\circ} \mathrm{F}\left(48.9^{\circ} \mathrm{C}\right)$. The MMP is 1466 psia (10.1 MPa).

use of the modified negative flash described here allowed direct calculation of the MMP. Fig. 5.8 shows the key tie line lengths as a function of system pressure, obtained by the algorithm described. Again the first crossover tie line controls miscibility. For displacement of oils containing dissolved $\mathrm{CH}_{4}, \mathrm{CO}_{2}$ will normally have the second highest $\mathrm{K}$-value, and hence the first crossover tie line will control miscibility (Johns and Orr [108]). The calculated MMP is 1466 psia (10.1 MPa). Again, the numerical results (see Fig. 5.7) confirm the analytical MMP with an estimate of 1460 psia (10.1 $\mathrm{MPa})$.

The algorithm given can be applied to a fully self-sharpening system with any number of components as long as the injection gas contains only a single component. This approach is more difficult to apply, however, if the injection gas contains more than one component. In the next section, we consider an alternative approach for such injection gas systems.

\subsubsection{Two-Component Gas Injection}

If the injection gas contains more than one component, then it is not necessarily true that a component disappears at each shock that connects two tie lines. Furthermore, the tie line intersection points associated with shock solutions cannot be determined a priori by using the fact that one component disappears at the intersection points. The example shown in Fig. 5.3 is such a system. Table 5.3 lists the oil and gas compositions as well as the system temperature. In this example, the injection gas contains $\mathrm{CO}_{2}$ and $\mathrm{CH}_{4}$, and the crossover tie line intersects both the initial and injection tie lines. However, the intersection points cannot be determined unless the crossover tie line is known. In principle, the technique for single-component gas injection could be used to determine the key tie lines if guesses of intersection points were made. However, experience indicates that the trial and error approach is difficult to apply in a way that converges routinely, 
Table 5.3: Oil and gas compositions for a four-component system, $\mathrm{T}=160^{\circ} \mathrm{F}$.

\begin{tabular}{|c||c|c|c|c|}
\hline \hline \multirow{2}{*}{ Component } & \multicolumn{4}{c|}{ Compositions(Mole Fraction) } \\
\cline { 2 - 5 } & $\mathrm{CH}_{4}$ & $\mathrm{CO}_{2}$ & $\mathrm{C}_{4}$ & $\mathrm{C}_{10}$ \\
\hline Oil & 0.20 & 0.00 & 0.15 & 0.65 \\
\hline Gas & 0.20 & 0.80 & 0.00 & 0.00 \\
\hline
\end{tabular}

especially when the system contains more than four components.

In such cases, therefore, it is more convenient to solve the full set of intersection equations simultaneously. In this four-component example, the equations for the two intersection points of the key tie lines are

$$
\begin{gathered}
c_{\mathrm{CH}_{4}, \mathrm{l}}^{o}\left[1+\left(K_{\mathrm{CH}_{4}}^{o}-1\right) S^{o}\right]=c_{\mathrm{CH}_{4}, 1}^{c}\left[\frac{K_{\mathrm{CH}_{4}}^{c}-K_{\mathrm{CO}_{2}}^{c}}{1-K_{\mathrm{CO}_{2}}^{c}}\right], \\
c_{\mathrm{C}_{4}, 1}^{o}\left[1+\left(K_{\mathrm{C}_{4}}^{o}-1\right) S^{o}\right]=c_{\mathrm{C}_{4}, 1}^{c}\left[\frac{K_{\mathrm{C}_{4}}^{c}-K_{\mathrm{CO}_{2}}^{c}}{1-K_{\mathrm{CO}_{2}}^{c}}\right], \\
c_{\mathrm{CH}_{4}, 1}^{g}\left[1+\left(K_{\mathrm{CH}_{4}}^{g}-1\right) S^{g}\right]=c_{\mathrm{CH}_{4}, 1}^{c}\left[\frac{K_{\mathrm{CH}_{4}}^{c}-K_{\mathrm{C}_{4}}^{c}}{1-K_{\mathrm{C}_{4}}^{c}}\right], \\
c_{\mathrm{CO}_{2}, 1}^{g}\left[1+\left(K_{\mathrm{CO}_{2}}^{g}-1\right) S^{g}\right]=c_{\mathrm{CO}_{2}, 1}^{c}\left[\frac{K_{\mathrm{CO}_{2}}^{c}-K_{\mathrm{C}_{4}}^{c}}{1-K_{\mathrm{C}_{4}}^{c}}\right],
\end{gathered}
$$

where $c_{i, l}^{o}$ are the liquid phase compositions of the initial tie line; $c_{i, l}^{g}$ are the liquid phase compositions of the injection gas tie line and $c_{i, l}^{c}$ are the liquid phase compositions of the crossover tie line; $K_{i}^{o}$ are the $\mathrm{K}$-values for the initial tie line; $K_{i}^{g}$ are the $\mathrm{K}$-values for the injection tie line and $K_{i}^{c}$ are the $\mathrm{K}$-values for the crossover tie line $\left(\mathrm{i}=\mathrm{CH}_{4}, \mathrm{CO}_{2}, \mathrm{C}_{4}, \mathrm{C}_{10}\right) . S^{o}$ is the vapor phase saturation on the initial oil tie line of the intersection point between the initial tie line and the crossover tie line, and $S^{g}$ is the vapor phase saturation on the injection gas tie line of the intersection point between the crossover tie line and the injection tie line.

The initial oil and injection gas tie lines can be found easily from the initial and injection compositions using the negative flash of Whitson and Michelsen [225] or the modified negative flash described here. The K-values of the crossover tie line are not known initially, but the equation of Wilson [226] or some other suitable guess can be used. For the example given here the equation of Wilson was used. Iteration over the K-values converged rapidly for the four-component example, and as the pressures were increased toward the MMP, the solution still converged fairly rapidly. Fig. 5.9 shows the tie line length as a function of the system pressure. It indicates that for this system the MMP is 2297 psia $(15.8 \mathrm{MPa})$, and the crossover tie line controls miscibility. The numerical simulation results shown in Fig. 5.7 confirm the analytical calculation with an estimated MMP of 2240 psia (15.4 MPa).

Fig. 5.10 shows that the effect of adding $\mathrm{CH}_{4}$ to $\mathrm{CO}_{2}$ is to increase the MMP substantially. When pure $\mathrm{CO}_{2}$ is injected, the crossover tie line lies in the $\mathrm{CO}_{2} / \mathrm{C}_{4} / \mathrm{C}_{10}$ face, and the MMP is easy to identify (Orr et al. [164]). As the $\mathrm{CH}_{4}$ fraction is increased the MMP climbs because the crossover tie line moves into the interior of the quaternary diagram, where the tie line is further from the critical locus at some pressure below the MMP. Hence, the pressure required to make the crossover tie line a critical tie line increases. When the $\mathrm{CH}_{4}$ fraction reaches $100 \%$ in the injection gas, the algorithm simply finds the critical tie line for the $\mathrm{CH}_{4} / \mathrm{C}_{4} / \mathrm{C}_{10}$ ternary system. 


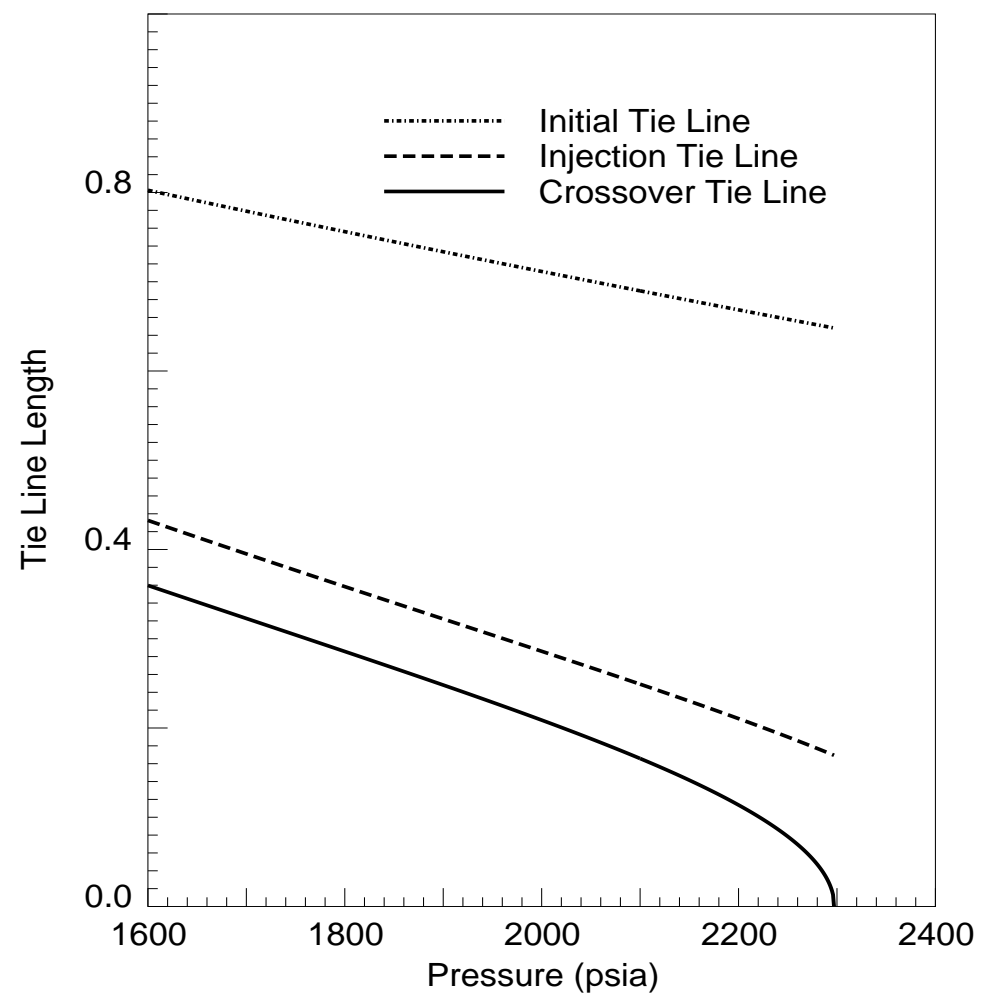

Figure 5.9: Key tie line lengths for a two-component gas displacement of a three-component oil at $160^{\circ} \mathrm{F}\left(71.1^{\circ} \mathrm{C}\right)$, (see Fig. 5.3). The MMP is 2297 psia (15.9 MPa).

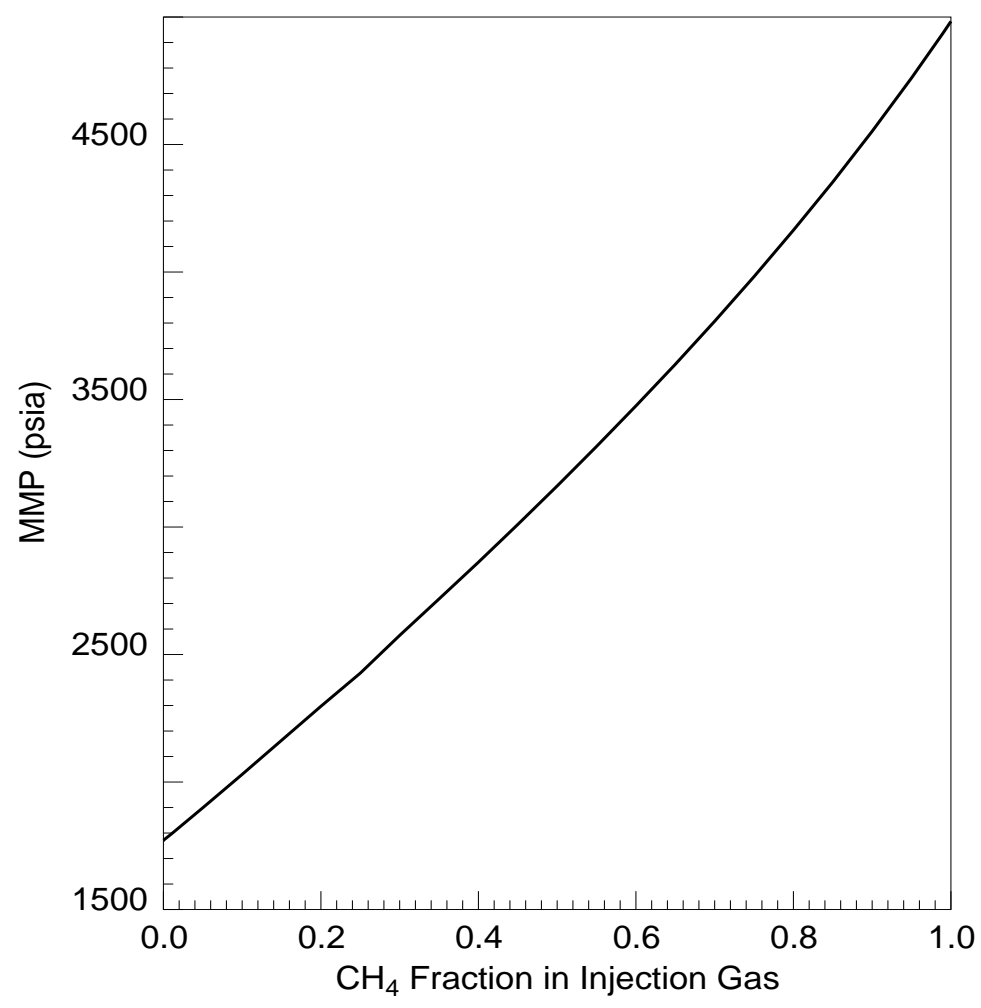

Figure 5.10: Effect of $\mathrm{CH}_{4}$ contamination of $\mathrm{CO}_{2}$ on $\mathrm{MMP}$ for the displacement of a $\mathrm{CH}_{4} / \mathrm{C}_{4} / \mathrm{C}_{10}$ mixture at $160^{\circ} \mathrm{F}\left(71.1^{\circ} \mathrm{C}\right)$. See Table 5.3 for the oil composition and Fig. 5.3 for the phase diagram. 


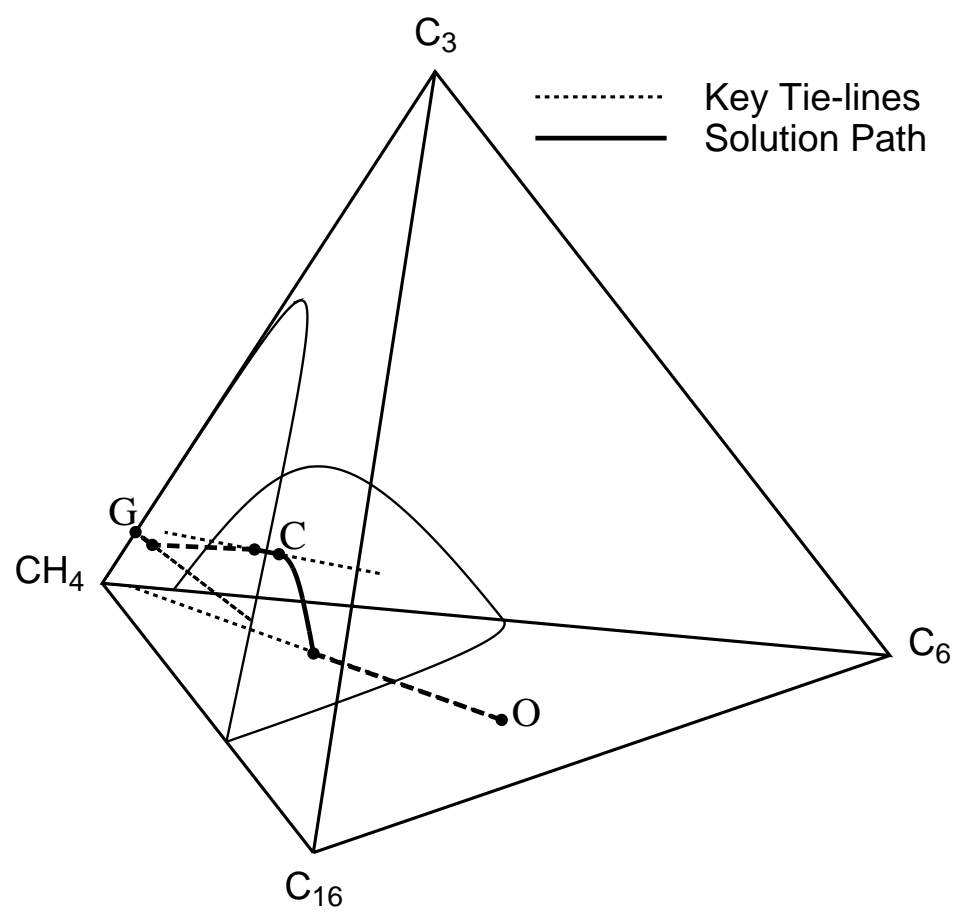

Figure 5.11: Displacement of $20 \% \mathrm{CH}_{4}, 40 \% \mathrm{C}_{6}$ and $40 \% \mathrm{C}_{16}$ by $90 \% \mathrm{CH}_{4}$ and $10 \% \mathrm{C}_{3}$ at $\mathrm{P}=$ 2000 psia and $\mathrm{T}=200^{\circ} \mathrm{F}$. There is a rarefaction between crossover tie line $\mathrm{C}$ and initial tie line $\mathrm{O}$.

\subsubsection{Nontie-line Rarefactions}

At least one additional issue must be addressed if analytical methods are to be used reliably to calculate MMPs for the full range of gas-oil system. While fully self-sharpening systems are quite common, it is possible to find exceptions. If the injection gas contains significant quantities of components with K-values less than one, then it is possible that some tie lines will be connected by rarefactions, and it is also possible to have rarefactions in other situations (see Johns et al. [109], for an example). The following example shows that even though tie lines are connected by rarefactions, the notion that all tie lines intersect each other is still a good approximation. If that is generally true then the MMP calculation method proposed in this report will still give accurate predictions of MMPs.

Fig. 5.11 shows a quaternary system in which a $\mathrm{CH}_{4} / \mathrm{C}_{3}$ mixture displaces a $\mathrm{CH}_{4} / \mathrm{C}_{6} / \mathrm{C}_{16}$ mixture. Direct application of the K-value rule suggested by Johns ([107]) indicates that there is a rarefaction along the nontie-line path connecting the crossover tie line and the initial tie line. To show that the notion of intersecting tie lines is still reasonable in this case, we first find the crossover tie line by solving the tie line intersection equations. Next we construct the analytical solution by integrating along the nontie-line path from the crossover tie line to the initial tie line. The numerical integration is quite simple. It consists of taking a small step along the nontie-line eigenvector direction, after which the eigenvector is evaluated again, and the process is repeated. When the composition steps taken are small, the numerical error associated with this simple integration is also small. In the calculations described here, the integration step size was $10^{-6}$. Finally, we compare our analytical solution with a solution obtained by fine-grid numerical simulation. To minimize the effect of numerical dispersion, we use a single-point upstream weighting scheme with 5000 grid blocks. Fig. 5.12 shows that the agreement between the analytical and numerical solutions is excellent. 

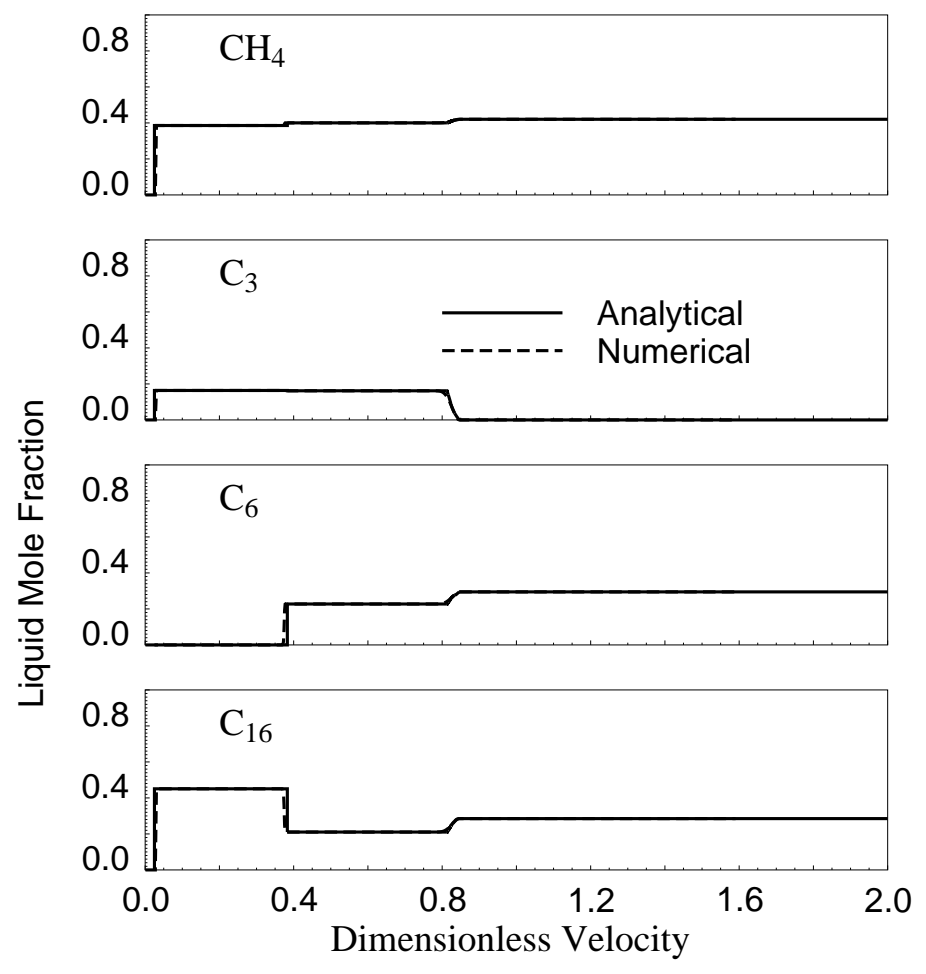

Figure 5.12: Numerical and analytical solutions for a system with a rarefaction along a nontie-line path.

Tables 5.4 and 5.5 compare two key tie lines obtained by three different approaches: analytical solution (Johns [107]), tie line intersection solution and numerical simulation. Table 5.4 compares crossover tie line. The analytical solution was found by integrating along a nontie-line path from the initial tie line to the crossover tie line. The numerical simulation version was obtained by conventional, fine-grid (5000 grid blocks), finite difference simulation. The tie line intersection version agrees quite closely (the maximum difference between tie lines is $4 \times 10^{-5}$ in the $\mathrm{C}_{6}$ liquid mole fraction). Table 5.5 compares initial oil tie lines obtained in a i different way. The analytical solution was obtained from a negative flash of the oil composition. The numerical version came from the compositional simulation. The tie line intersection result was obtained by integrating along the nontie-line path from the crossover tie line (calculated with the intersection equations) to the $\mathrm{C}_{3}=0$ face. Here again, the maximum difference in phase compositions is about $3 \times 10^{-5}$ $\left(\mathrm{C}_{6}\right.$ mole fraction in the liquid). That difference is small enough to be consistent with the accuracy of the numerical integration along the nontie-line path (step size $10^{-6}$ ). Thus, we conclude that it is reasonable to use the tie line intersection method to estimate the MMP even in the absence of a proof that tie lines intersect when nontie-line rarefactions are present. For the particular system shown in Fig. 5.11, we use the tie line intersection method to calculate the MMP. Fig. 5.13 shows the MMP is 4264 psia (29.4 MPa). Numerical simulation results similar to those shown in Fig. 5.7 gave an estimate of $4320 \mathrm{psia}(29.8 \mathrm{MPa})$ for the MMP. In this example, therefore, the estimate of the MMP from tie line intersection approach is accurate.

Computation times for MMP calculations described here are compared in Table 5.6. Estimation of the MMP by compositional simulation requires that simulations be performed at multiple pressures (we used 10 pressures for the examples in Fig. 5.7). For the relatively simple systems considered here, the tie line intersection approach is about two orders of magnitude faster than 


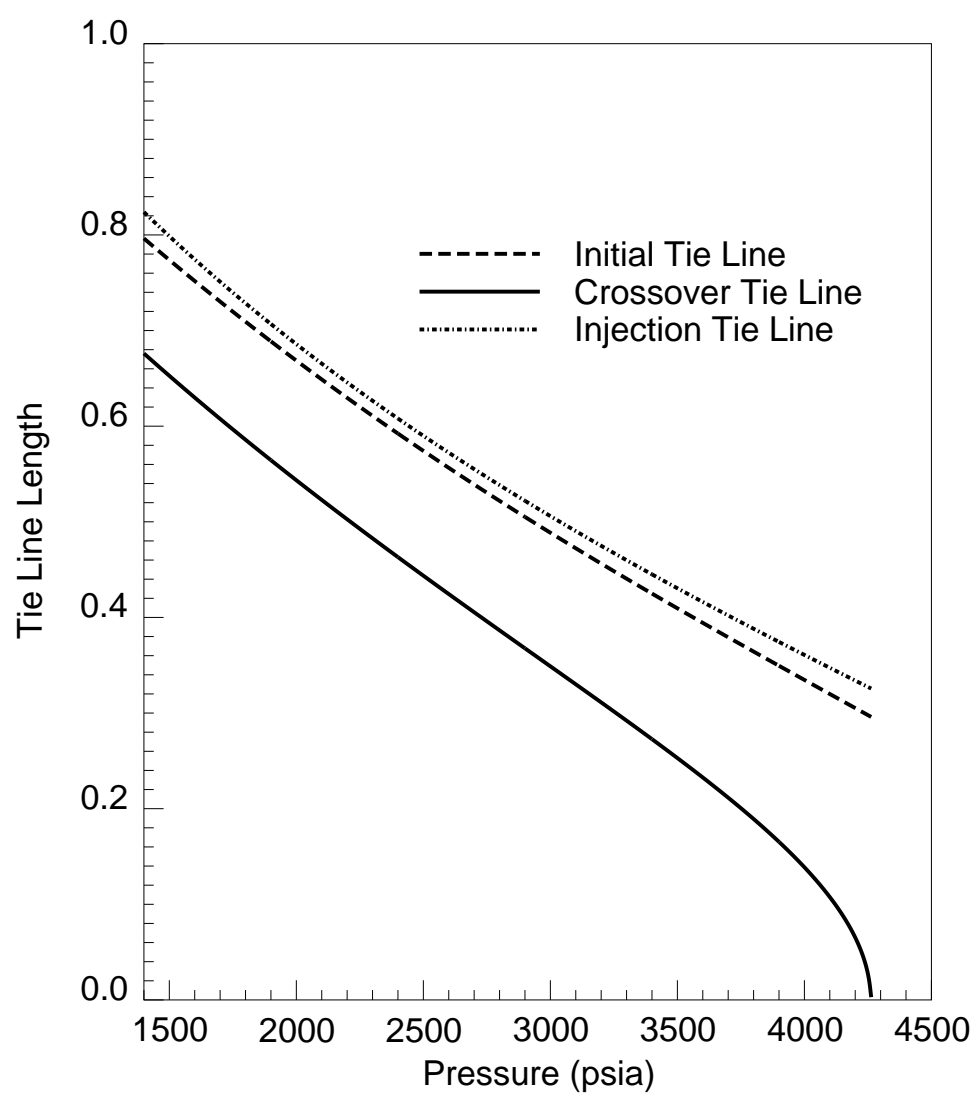

Figure 5.13: Displacement of $20 \% \mathrm{CH}_{4}, 40 \% \mathrm{C}_{6}$ and $40 \% \mathrm{C}_{16}$ by $90 \% \mathrm{CH}_{4}$ and $10 \% \mathrm{C}_{3}$ at $\mathrm{T}=$ $200^{\circ} \mathrm{F} . \mathrm{MMP}=4264 \mathrm{psia}(29.4 \mathrm{MPa})$.

conventional simulation. For systems with more components present, the compositional simulation times will grow rapidly with the number of components. Hence, we argue that the tie line intersection technique offers a significant advantage over conventional simulation.

\subsection{Conclusions}

The analysis and examples reported here demonstrate that:

1. A modified negative flash calculation can be performed that yields convergent solutions for composition points far outside the two-phase region, for compositions outside the phase diagram, and for compositions on extensions of near-critical tie-lines.

2. For fully self-sharpening systems in which a gas containing one component displaces a multicomponent oil, the modified negative flash can be used to find the sequence of key tie lines from the fact that one component disappears across each shock. The MMP is the lowest pressure at which one of the key tie lines is a critical tie line.

3. For fully self-sharpening systems in which a gas containing two components displaces a fourcomponent oil, the crossover tie line can be found by solving the full set of tie line intersection equations simultaneously. The MMP can then be found as the lowest pressure at which one of the key tie lines is a critical tie line.

4. For systems that are not fully self-sharpening, it is still reasonable to assume that all key tie lines intersect each other. The same approach can then be used to calculate the MMP. 
Table 5.4: Tie-line structure for two-component injection.

\begin{tabular}{|c||c|c|c|c|c|c|}
\hline \hline \multirow{2}{*}{ Tie-lines } & \multicolumn{7}{|c|}{ Compositions } \\
\cline { 2 - 7 } & $\mathrm{CH}_{4}$ & $\mathrm{CO}_{2}$ & $\mathrm{C}_{4}$ & $\mathrm{C}_{10}$ & $\mathrm{C}_{14}$ & $\mathrm{C}_{20}$ \\
\hline Initial & $\sqrt{ }$ & & $\sqrt{ }$ & $\sqrt{ }$ & $\sqrt{ }$ & $\sqrt{ }$ \\
\hline Crossover 1 & $\sqrt{ }$ & $\sqrt{ }$ & $\sqrt{ }$ & $\sqrt{ }$ & $\sqrt{ }$ & $\sqrt{ }$ \\
\hline Crossover 2 & $\sqrt{ }$ & $\sqrt{ }$ & & $\sqrt{ }$ & $\sqrt{ }$ & $\sqrt{ }$ \\
\hline Crossover 3 & $\sqrt{ }$ & $\sqrt{ }$ & & & $\sqrt{ }$ & $\sqrt{ }$ \\
\hline Injection & $\sqrt{ }$ & $\sqrt{ }$ & & & & $\sqrt{ }$ \\
\hline
\end{tabular}

Table 5.5: Comparison of the initial tie lines obtained from three different approaches for the system shown in Fig. 5.11.

\begin{tabular}{|c||c|c|c|c|}
\hline \hline \multicolumn{1}{|c||}{ Initial Tie Line } & \multicolumn{4}{c|}{ Liquid Phase Mole Fraction } \\
\cline { 2 - 5 } & $\mathrm{CH}_{4}$ & $C_{3}$ & $C_{6}$ & $C_{16}$ \\
\hline Analytical & 0.420225 & 0.000000 & 0.294695 & 0.285080 \\
\hline Numerical & 0.420260 & 0.000000 & 0.294660 & 0.285080 \\
\hline Tie Line Intersection & 0.420224 & 0.000000 & 0.294674 & 0.285102 \\
\hline \multicolumn{1}{|c||}{} & \multicolumn{4}{c|}{ Vapor Phase Mole Fraction } \\
\hline Analytical & 0.965949 & 0.000000 & 0.033674 & 0.000377 \\
\hline Numerical & 0.965943 & 0.000000 & 0.033679 & 0.000378 \\
\hline Tie Line Intersection & 0.965952 & 0.000000 & 0.033671 & 0.000377 \\
\hline
\end{tabular}

Table 5.6: Comparison of CPU times between the numerical and analytical approaches.

\begin{tabular}{|c||c|c|c|c|}
\hline \hline \multicolumn{1}{|c||}{} & \multicolumn{4}{c|}{ System } \\
\cline { 2 - 5 } Figure & Fig. 9 & Fig. 13 & Fig. 6 & Fig. 8 \\
\hline Number of Components & 4 & 4 & 6 & 11 \\
\hline Number of Pressures & 10 & 10 & 10 & 10 \\
\hline Method & \multicolumn{5}{c|}{ CPU Time } \\
\hline Numerical Simulation & $11.2 \mathrm{hrs}$ & $10.4 \mathrm{hrs}$ & $19.1 \mathrm{hrs}$ & $32.1 \mathrm{hrs}$ \\
\hline Analytical Approach & $1.3 \mathrm{mins}$ & $2.8 \mathrm{mins}$ & $2.0 \mathrm{mins}$ & $3.5 \mathrm{mins}$ \\
\hline
\end{tabular}




\title{
6. Calculation of Minimum Miscibility Pressure for Multicomponent Injection Gases
}

\author{
Yun Wang and Franklin M. Orr, Jr.
}

\subsection{Abstract}

We describe and test a method for calculation of minimum miscibility pressure (MMP) that makes use of an analytical theory for one-dimensional, dispersion-free flow of multicomponent mixtures. The theory shows that in a displacement of an oil by a gas with $n_{c}$ components, the behavior of the displacement is controlled by a sequence of $n_{c}-1$ key tie lines. Besides the tie lines that extend through the initial oil and injection gas compositions, there are $n_{c}-3$ tie lines, known as crossover tie lines, that can be found from a set of conditions that requires the extensions of the appropriate tie lines to intersect each other. The MMP is calculated as the pressure at which one of the key tie lines becomes a critical tie line (a tie line of zero length that is tangent to the critical locus). We describe the numerical approach for solving the tie line intersection equations, and we use slim tube test and compositional simulation data reported in the literature to show that the proposed approach can be used to calculate MMP accurately for displacements with an arbitrary number of components present.

\subsection{Introduction}

Design of a multicontact miscible gas injection project requires knowledge of the minimum miscibility pressure (or minimum gas enrichment), the pressure (or enrichment) above which high pressure gas displaces nearly $100 \%$ of oil in the swept zone. In this chapter we examine methods for calculating the MMP from an equation-of-state based representation of phase behavior for mixtures of the oil displaced with the injected gas.

The earliest attempts to calculate MMPs were based on ternary theory for multicontact miscible displacement ([100]). For example Benham [15] constructed approximate ternary diagrams and used extensions of critical tie lines to estimate minimum enrichments for condensing gas drive systems. Metcalfe et al. [138] described a method for condensing gas drives based on multiple equilibrium mixing cells. In that method, a sort of simplified finite difference compositional simulation, gas was mixed with oil in the first cell and flashed. Liquid and vapor in excess of the cell volume were moved downstream to the next cell, and the process repeated. The MMP was determined as the pressure required to produce near-critical mixtures in downstream cells. Kuo [121] used a single cell version of the mixing cell approach, again for condensing gas drives. In his method, gas was mixed with oil and flashed. The resulting vapor was removed and fresh injection gas added to the liquid in the cell (sometimes called reverse contacts), and the flash calculation was repeated. The mixing process was then repeated until the liquid phase composition converged. If it converged to a critical mixture (vapor and liquid identical), the system was taken to be miscible. If not the pressure was incremented and the process repeated to find the MMP. Luks et al. [129] used a similar technique for both condensing and vaporizing drives. For vaporizing gas drives, vapor from each flash was mixed with fresh oil (forward contacts). Both forward and reverse contacts were

\footnotetext{
${ }^{5}$ The material in this chapter was presented at the 1998 SPE/DOE Improved Oil Recovery Symposium in Tulsa, Oklahoma, April 19-22, 1998, and is published as SPE paper 39683 in the conference proceedings.
} 
performed, and the MMP was taken to be the lower of the pressures required to produce a critical mixture by repeated contacts.

Nouar and Flock [153] used the properties of ternary systems in a different way. They calculated critical compositions and tie line slopes directly (for ternary systems only) and used them to estimate MMP for vaporizing gas drive systems. The MMP was taken to be the pressure at which the oil composition was intersected by the extension of the critical tie line. Systems with more than three components were handled by correlating calculated ternary MMPs with variable molecular weight for the intermediate and heavy hydrocarbon components.

Thus, the behavior of ternary systems is well understood. The MMP can be determined by finding the pressure at which the oil composition (for a vaporizing gas drive), or the injected gas composition (for a condensing gas drive), is located on the extension of a critical tie line. That tie line can be found directly by one of the mixing cell methods or by performing negative flash calculations (Whitson and Michelsen [225], Wang and Orr [220]).

It is now known, however, that extension of these ideas to systems with more than three components requires additional understanding. Zick [230] showed that multicomponent displacements can display features of both condensing and vaporizing drives. He also showed that the MMP could be estimated by one-dimensional compositional simulation, provided sufficient care was taken to account for the effects of numerical dispersion. A subsequent paper by Stalkup [198] confirmed Zick's results and outlined a procedure for extrapolation of the number of grid blocks to eliminate effects of numerical dispersion. The papers by Zick and Stalkup demonstrate clearly that the MMP can always be found by compositional simulation, though fine grids and repeated simulations at multiple pressures will mean that significant computation times will be required.

Jensen and Michelsen [102] continued the investigation of the ideas of critical tie lines from ternary systems. They showed that for systems with more than three components, the pressures required to force critical tie lines to extend through the injection gas composition or the oil composition do not necessarily indicate the correct MMP. They advocated use of a mixing cell approach instead. (We will show below that the single cell technique can also lead to inaccurate MMPs for that system.) Given the speed and simplicity of single cell methods, investigations of their properties have continued ([148]), and multicell methods have also been examined ([175]).

The reason for the inaccuracies encountered in the critical tie line and single cell methods became apparent when analytical solutions were obtained for one-dimensional, dispersion-free flow of four-component mixtures. Monroe et al. [143] showed that three key tie lines control displacement behavior in four-component systems: the tie line that extends through injection gas composition, the tie line that extends through the oil composition, and a third tie line called "the crossover tie line". Orr et al. [163] then showed that multicontact miscibility develops if any of the three key tie lines is a critical tie line. In many displacements, it is the crossover tie line that approaches the critical locus at the lowest pressure, and in such cases that tie line determines the MMP. Johns et al. [109] then showed that the condensing/vaporizing mechanism described by Zick [230] arises when a crossover tie line controls development of miscibility. Hence, methods that identify only the critical tie lines associated with the injection gas or the initial oil are doomed to failure for condensing/vaporizing systems.

The analytical approach was used by Dindoruk et al. [45] to study MMP behavior of $\mathrm{N}_{2}$ systems, and the application to systems with more than four components was considered by Johns and Orr [108]. In systems with $n_{c}$ components, there are $n_{c}-3$ crossover tie lines, and the initial oil and injection gas tie lines are also still important. (In certain special cases, a degenerate solution can occur when two key tie lines coincide and the system is described by $n_{c}-2$ key tie lines. We give an example below.) Johns and Orr considered only a single-component injection gas $\left(\mathrm{CO}_{2}\right)$. They made use of the fact that in many cases, the crossover tie lines can be found from a geometric construction that arises from the properties of shocks in the analytical solutions ([109]) (when two 
tie lines are connected by a shock, their extensions must intersect). For example, Fig. 6.1 shows a four component system (methane carbon dioxide, butane and decane) in which all key tie lines are connected by shocks. The crossover tie line is the only tie line in the interior of the quaternary diagram that (when extended at both ends) intersects the extensions of the tie lines that extend through injection gas and initial oil compositions. Wang and Orr [220] showed how to solve the tie-line intersection equations efficiently for a single-component injection gas displacing an oil with an arbitrary number of components. They also considered displacement by a two-component gas, but only for four-component systems.

In this chapter we extend the work of Johns and Orr [108] and Wang and Orr [220] to systems with an arbitrary number of components in either the initial oil or the injection gas. We begin with a description of the tie line intersection approach and then we report results of a series of tests of the accuracy of the technique.

\subsection{Mathematical Approach}

Johns et al. [109] showed, based on their analytical solutions, that if any two tie lines are connected by a shock, the extensions of the tie lines must intersect (see Wang and Orr [220] for a discussion of self-sharpening composition variations that produce shocks and spreading composition variations, sometimes called rarefactions, that do not). For displacements in which all the tie lines are connected by shocks, then, the $n_{c}-1$ key tie lines can be found rigorously as follows. The initial oil and injection gas tie lines are found using the negative flash of Whitson and Michelsen [225] or the modified negative flash of Wang and Orr [220]. The $n_{c}-3$ crossover tie lines are then found from the conditions that each crossover tie line intersects the tie line just upstream and the tie line just downstream. Once the key tie lines have been found at a given pressure, the MMP can be determined by increasing the pressure in increments until one of the key tie lines becomes a critical tie line. This approach is completely rigorous if the displacement system is fully self-sharpening (see Wang and Orr [220] for a discussion of how self-sharpening behavior is detected). If the displacement path contains one or more continuous variations between tie lines (rarefactions) then the tie lines obtained by the intersection method are an approximation to the actual key tie lines, but all evidence obtained to date indicates that the approximation is a very good one (see Wang and Orr [220]). Thus, the tie line intersection method can be used to estimate MMPs, whether or not the displacement system is fully self-sharpening, and the calculated MMPs will be accurate if the characterization of the gas/oil system gives an accurate representation of phase behavior in the neighborhood of the critical locus.

Any pair of tie lines that intersect must satisfy

$$
x_{j}^{n}\left[1+\left(K_{j}^{n}-1\right) V_{n}^{n}\right]=x_{j}^{n+1}\left[1+\left(K_{j}^{n+1}-1\right) V_{n}^{n+1}\right],
$$

where $n=1, n_{c}-2 ; j=1, n_{c}-1$.

In Eqs. $6.1, x_{j}^{n}$ is the liquid phase mole fraction for component $j$ on tie line $n, K_{j}^{n}$ is the equilibrium $\mathrm{K}$-value for component $j$ on tie line $n, V_{n}^{n}$ is the vapor phase mole fraction on tie line $n$ of the intersection point between tie line $n$ and tie line $n+1$ and $V_{n}^{n+1}$ is the vapor phase mole fraction on tie line $n+1$ of the intersection point between tie line $n$ and tie line $n+1$. In Eqs. 6.1, the initial oil tie line and the injection gas tie lines are known. They can be obtained by performing negative flashes for the initial oil and injection gas compositions. Elimination of vapor phase mole fractions from Eqs. 6.1 leaves liquid phase compositions as unknowns. If there is no component missing across two intersecting tie lines, the vapor phase mole fractions in Eqs. 6.1 are

$$
V_{n}^{n}=\frac{a_{1}^{n}-a_{2}^{n}}{b_{1}-b_{2}},
$$


and

$$
V_{n}^{n+1}=\frac{a_{1}^{n+1}-a_{2}^{n+1}}{b_{1}-b_{2}}
$$

where

$$
\begin{gathered}
a_{1}^{n}=\left(x_{1}^{n+1}-x_{1}^{n}\right) x_{2}^{n+1}\left(K_{2}^{n+1}-1\right), \\
a_{1}^{n+1}=\left(x_{1}^{n+1}-x_{1}^{n}\right) x_{2}^{n}\left(K_{2}^{n}-1\right), \\
a_{2}^{n}=\left(x_{2}^{n+1}-x_{2}^{n}\right) x_{1}^{n+1}\left(K_{1}^{n+1}-1\right), \\
a_{2}^{n+1}=\left(x_{2}^{n+1}-x_{2}^{n}\right) x_{1}^{n}\left(K_{1}^{n}-1\right), \\
b_{1}=x_{2}^{n+1} x_{1}^{n}\left(K_{2}^{n+1}-1\right)\left(K_{1}^{n}-1\right),
\end{gathered}
$$

and

$$
b_{2}=x_{1}^{n+1} x_{2}^{n}\left(K_{1}^{n+1}-1\right)\left(K_{2}^{n}-1\right) .
$$

If there is one component missing on one of the two intersecting tie lines, the vapor mole fractions in Eqs. 6.1 are

$$
V_{n}^{n}=\frac{1}{1-K_{n+1}^{n}}
$$

and

$$
V_{n}^{n+1}=\frac{1}{K_{1}^{n+1}-1}\left[\frac{x_{1}^{n}}{x_{1}^{n+1}}\left(\frac{K_{1}^{n}-K_{n+1}^{n}}{1-K_{n+1}^{n}}\right)-1\right]
$$

Substitution of vapor mole fractions given by Eqs. 6.2 and 6.3 or by Eqs. 6.10 and 6.11 into Eqs. 6.1, along with the constraint that liquid and vapor phase mole fractions must sum to one,

$$
\sum_{i=1}^{n_{c}-1}\left(K_{i}^{n}-K_{n_{c}}^{n}\right) x_{i}^{n}=\left(1-K_{n_{c}}^{n}\right)
$$

gives a system of nonlinear equations that can be solved to find the intersecting tie lines.

The tie lines obtained by solution of the intersection equations can then be tested for closeness to the critical locus. In particular, the length of a tie line goes to zero as it approaches the critical locus with increasing pressure, for example. The line length, for a particular tie line $n$ is defined as

$$
L_{n}=\sqrt{\sum_{i=1}^{n_{c}}\left(y_{i}^{n}-x_{i}^{n}\right)^{2}}
$$

where $y_{i}^{n}$ is the vapor phase mole fraction for component $i$ on tie line $n$.

The algorithm used to solve the intersection equations at each pressure is: 
1. For a given set of K-values, solve Eqs. 6.1 by Newton-Raphson iteration to obtain a set of intersecting tie lines in terms of the liquid phase mole fractions along each key tie lines.

2. Update K-values using one step of the accelerated successive substitution scheme of Mehra et al. [136].

3. Repeat steps 1-2 to convergence.

The algorithm for calculating the MMP is:

1. Begin with a relatively low pressure. Perform a negative flash to find the initial and injection tie lines at the low pressure.

2. Find the tie line intersection solution for the low pressure using the steps outlined in the Appendix.

3. Increase the pressure slightly and use the solution obtained in step 2 as the initial guess to solve the tie line intersection equations.

4. Repeat step 3 until one of the key tie lines shrinks to a point. The pressure at which some key tie line has zero length is the MMP.

This approach to solving for the key tie lines can be applied to systems with an arbitrary number of components in the oil or gas. If convergent solutions can be found for the intersection equations, the resulting MMP will be rigorously accurate if all the tie lines that make up the solution to the conservation equations are connected by shocks (Johns and Orr [108], Wang and Orr [220]). Even if rarefactions (continuous variations between key tie lines) are present, the error induced by the assumption of tie line intersections appears to be quite small (Wang and Orr [220]).

\subsection{Examples of MMP Calculations}

\subsubsection{Four-component System: $\mathrm{CH}_{4} / \mathrm{CO}_{2} / \mathrm{C}_{4} / \mathrm{C}_{10}$}

We begin the evaluation of accuracy of the tie line intersection method with a calculation of MMPs for the $\mathrm{CH}_{4} / \mathrm{CO}_{2} / \mathrm{C}_{4} / \mathrm{C}_{10}$ system considered by Jensen and Michelsen [102]. The oil mixture contains $40 \% \mathrm{CH}_{4}, 5 \% \mathrm{CO}_{2}, 20 \% \mathrm{C}_{4}$ and $35 \% \mathrm{C}_{10}$, and the displacement temperature is $71^{\circ} \mathrm{C}\left(160^{\circ} \mathrm{F}\right)$. Fig. 6.2 compares MMPs calculated by the tie line intersection approach to those reported by Jensen and Michelsen for mixtures of $\mathrm{CH}_{4}$ and $\mathrm{CO}_{2}$. Jensen and Michelsen used the Soave-Redlich-Kwong (SRK) equation of state and hence we do as well for the results shown in Fig. 6.2 only. In other examples described below, the Peng-Robinson equation (PREOS) was used.

Jensen and Michelsen demonstrated that the MMP estimated as the pressure at which the initial oil tie line became a critical tie line was not appropriate. They also showed that a single-cell mixing cell calculation based on forward contacts generated critical tie lines at pressures close to the pressure required for first-contact miscibility. However, for $\mathrm{CO}_{2}$ mole fractions exceeding $6.6 \%$ in the injection gas, the single-cell estimate is 200-350 psia higher than the MMP calculated here. Fig. 6.3 illustrates why (Fig. 6.3 was generated with the PREOS, but the patterns are exactly the same with the SRK equation). Consider first what would happen if no $\mathrm{CO}_{2}$ were present in the initial oil and pure methane were injected. In that ternary system the MMP would be the pressure at which the oil mixture lies on the extension of the critical tie line in the $\mathrm{CH}_{4} / \mathrm{C}_{4} / \mathrm{C}_{10}$ face. Thus in that system, the initial oil tie line does determine the MMP.

Now consider the initial oil mixture of Jensen and Michelsen, which contains $5 \% \mathrm{CO}_{2}$. Fig. 6.3 shows the key tie lines for pure $\mathrm{CH}_{4}$ injection. In this case, the initial oil tie line moves into the 
interior of the quaternary diagram, but the crossover tie line remains in the $\mathrm{CH}_{4} / \mathrm{C}_{4} / \mathrm{C}_{10}$ face. As the pressure is increased, the two-phase region shrinks, and the initial oil tie line approaches the critical locus first as the pressure increases. Thus, the initial oil tie line still determines the MMP for pure $\mathrm{CH}_{4}$ injection.

Next, consider what happens as $\mathrm{CO}_{2}$ is added to the injection gas. As the amount of $\mathrm{CO}_{2}$ in the injection gas increases, the crossover tie line moves up the vertical surface shown in Fig. 6.3 . When the injection gas contains $6.6 \% \mathrm{CO}_{2}$, the crossover tie line coincides with the initial oil tie line. (This is an example of a degenerate case in which there are only two key tie lines.) For injection gas mixtures containing less than $6.6 \% \mathrm{CO}_{2}$, the initial oil tie line still controls development of miscibility, and the MMP remains unchanged (see Fig. 6.2). When the $\mathrm{CO}_{2}$ fraction is larger than $6.6 \%$, however, the situation changes. Now the crossover tie line lies above the initial tie line on the vertical surface shown in Fig. 6.3. The crossover tie line now approaches the critical locus at a lower pressure than does the oil tie line, and the crossover tie line determines miscibility. (These crossover tie lines are determined entirely by shocks, so the tie line intersection method is rigorously correct when the $\mathrm{CO}_{2}$ fraction in the injection gas exceeds $6.6 \%$ ). As the $\mathrm{CO}_{2}$ fraction increases, the crossover tie line moves closer to the critical locus because the two-phase region is smaller at higher $\mathrm{CO}_{2}$ fractions. Hence, the pressure increase required to move the critical locus to reach the crossover tie line decreases. The result is lower MMPs with increasing $\mathrm{CO}_{2}$ fraction in the injection gas. Thus, the MMP is independent of $\mathrm{CO}_{2}$ fraction at low $\mathrm{CO}_{2}$ fraction (less than $6.6 \%$ ) in the injection gas and relatively sensitive to $\mathrm{CO}_{2}$ fraction at higher $\mathrm{CO}_{2}$ fractions. Similar behavior was described by Dindoruk et al. ([45]) for injection of $\mathrm{N}_{2} / \mathrm{CH}_{4}$ mixtures.

To confirm the MMP calculated by the tie line intersection method, the MMP was also estimated by compositional simulation for $50 \% \mathrm{CO}_{2}$ in the injection gas. Fig. 6.4 shows the results of simulations with 100, 200, and 500 grid blocks, along with extrapolated recoveries at 1.2 pore volumes injected. The simulation estimate of 2500 psia obtained by extrapolation as described by Stalkup [198] agrees well with the analytical value of 2557 psia (using PREOS).

So why does the single cell calculation technique fail to produce the correct MMP over the full range of injection gas compositions? Fig. 6.5 shows the composition path of mixtures obtained by (1) mixing $80 \%$ injection gas with $20 \%$ oil and then (2) mixing the vapor from each contact with $20 \%$ fresh oil. The calculations were performed at the MMP of 2557 psia obtained by the tie line intersection calculation. The sequence of tie lines converges to the tie line that extends through the initial oil composition. Similarly, reverse contacts (fresh injection gas mixed with equilibrium liquid at each stage) converge to the tie line that extends through the injection gas composition. Thus, the single cell contacts do identify two of the $n_{c}-1$ key tie lines. However, neither forward nor reverse contacts identify the crossover tie line, which is the tie line that becomes a critical tie line in this condensing/vaporizing gas drive. If the pressure is increased substantially above the actual MMP, it is possible for the forward contact procedure to generate tie lines close to the critical locus, as Jensen and Michelsen [102] showed, but that pressure is not an accurate estimate of the MMP. Fig. 6.2 shows that the MMPs calculated by forward contacts are higher (200-350 psia) than the MMPs calculated by the tie line intersection method in the region where the crossover tie line controls miscibility. Apparently, the first few forward contacts enrich the vapor phase enough in intermediate components that subsequent mixtures pass close to the critical locus. For example, the values reported by Jensen and Michelsen for forward contact MMPs are close to the pressure required for first-contact miscibility with the gas, pressures that are significantly higher than the actual MMP. Thus, we conclude that calculations based on single mixing cell should not be used to estimate MMPs if there is any chance that miscibility is determined by a tie line other than the initial oil or injection gas tie lines, the situation that exists in condensing/vaporizing drives $([230][109])$

Hearn and Whitson [83] state, however, that a multiple mixing cell method ([138]) can be 
used for such systems and report MMPs calculated by an unpublished method attributed to Zick. Multiple cell methods have much in common with compositional simulation, which does identify the appropriate tie lines by solving the conservation equations numerically and therefore gives MMPs consistent with the tie line intersection approach, as long as care is taken to account for numerical dispersion. Hence, it is reasonable to assume that an appropriate computational technique based on multiple mixing cells could be devised to substitute for calculations based on single mixing cells.

\subsubsection{Crude Oil Systems}

To examine how the tie line intersection method works for crude oil displacements we consider four examples described by Hearn and Whitson [83]. They reported results of slim tube experiments performed for lean and rich gas displacements of crude oil from the Safah field, Oman at $212^{\circ} \mathrm{F}\left(100^{\circ} \mathrm{C}\right)$. The oil and injected gas compositions are listed in Tables 1 and 2 . Two sets of EOS phase behavior characterization were reported: a 15-component characterization and an 8-component characterization. Hearn and Whitson reported compositional simulation results and MMPs calculated by Zick's technique based on multiple mixing cells in addition to slim tube data.

Results of the analytical calculations for the rich gas displacement of the 8-component oil are shown in Fig. 6.6, which reports the distribution of key tie line lengths as a function of displacement pressure. With increasing pressure, the fourth crossover tie line shrinks to a point at a pressure of 3279 psia, therefore the MMP for this system is 3279 psia.

Slim tube data reported by Hearn and Whitson are compared with various calculated MMPs in Table 3. For the rich gas drive, the pressure increment between displacements was large enough (1000 psia), that the MMP was not determined with high precision in the experiment. Hearn and Whitson apparently used compositional simulation results to estimate the slope of the plot of recovery versus pressure, arriving at an estimate of $3300 \pm 100$ psig for the MMP. They also reported a calculated MMP of 3190 psig obtained for the 8-component representation. That value was obtained both by compositional simulation and by the multiple mixing cell technique. The MMP value reported here, 3279 psia, is therefore in good agreement with both experimental data and calculations reported by Hearn and Whitson.

Results of the analytical calculations for the lean gas drive test of the 8-component characterization are shown in Fig. 6.7. Again, as the displacement pressure increases, the lengths of all the key tie lines decrease as the two-phase region shrinks. The MMP obtained for the 8-component representation was 5582 psia. In this case as well, there is considerable uncertainty in the experimental MMP. Only two slim tube tests were performed, at 2500 and 4500 psia. Neither was miscible. Linear extrapolation of the recovery data to $100 \%$ recovery (at 1.2 pore volumes injected) gives an estimate of 5000 psig, the value reported in the text of paper by Hearn and Whitson. Extrapolation from the data point at 4500 psia with the slope of the recovery curve obtained from compositional simulation gives an estimate of $5200 \mathrm{psig}$, the value reported in Table 3 of the paper. The calculated value reported by Hearn and Whitson was 5865 psig for the 8-component model. That value was reported both for calculations by compositional simulation and for forward contacts in a single mixing cell.

In our calculations, it is the second crossover tie line that determines the MMP, though the first crossover tie line is also nearly critical. It is possible that the MMP (5865 psia) obtained by the forward contact mixing cell calculation reported by Hearn and Whitson is higher than that obtained here because the mixing cell method finds the initial tie line, which requires a higher pressure to become a critical tie line. In our implementation of the Peng-Robison EOS, however, that pressure is 6163 psia. It is less clear why the compositional simulation result is exactly the same as the mixing cell result, though small uncertainties in the extrapolation to zero dispersion may be responsible. In any case, the calculated values are reasonably consistent with the experimental 
observations, though it appears that the fluid characterization chosen by Hearn and Whitson gives an MMP that is a bit higher than the experimental result, whatever calculation method is used.

Fig. 6.8 and Fig. 6.9 are the results for the rich and lean gas drive tests respectively, using the 15-component characterization reported by Hearn and Whitson. Fig. 6.8 shows that the calculated MMP for the rich gas drive is 3115 psia. It is now the 8th crossover tie line that controls miscibility. Although the MMP is slightly lower than the calculated value using the 8-component characterization, it is still in good agreement with the slim tube result of 3300 psig. Fig. 6.9 shows the MMP for the lean gas drive is 5253 psia. In this example, the 15-component characterization resulted in better agreement of the calculated and experimental MMPs. Compared to 5582 psia of the 8-component estimate, the calculated MMP of 5253 psia for the 15-component characterization is closer to the slim tube MMP of 5200 psig.

Next we consider an example system described by Zick [230]. Table 4 shows the compositions of the oil and injected gas. The injection gas is a five-component mixture labeled as solvent A in Zick's paper (Gas 1 in Table 4), and the oil is reservoir fluid A (12-component Oil 1 in Table 4). The initial oil composition was obtained by flashing reservoir fluid A at 1500 psig. The resulting liquid phase was taken as the oil. The displacement calculations were performed at the reservoir temperature of $185^{\circ} \mathrm{F}$. Fig. 6.10 shows the key tie line length distribution. At 2169 psia, the fourth crossover tie line becomes a critical tie line. The slim tube data showed an apparent MMP of 2200 psig. In this system as well, the agreement between calculated and experimental MMPs is good. We also calculated an MMP for a mixture of $20 \%$ methane plus $80 \%$ solvent A (Gas 2 in Table 4). In this case the initial oil was obtained by flashing reservoir fluid A at $3000 \mathrm{psig}$ (Oil 2). The experimental MMP in this case was 3100 psig. Fig. 6.11 shows the resulting tie line lengths obtained in the calculation of tie line intersections. At around 2817 psia, the third and fourth crossover tie lines become parallel and the tie line intersection solution becomes singular. If large pressure steps are used, the solution sequence will step over the singularity, provided that the solution converges. If the pressure sequence is close to the MMP (and hence some tie line is near the critical locus), convergence difficulties can occur. In such cases, we used a third order extrapolation scheme to obtain a reasonably good estimate of liquid phase compositions as well as K-values for the next pressure step. As it turns out, the singularity is not uncommon, especially when the oil and gas contain a large number of components, some of which have similar K-values. For such systems, a different version of the intersection equations suggested by Jessen et al. [105] avoids difficulties that arise from coinciding tie lines. As the results indicate, the calculated MMP of 3013 psia is in good agreement with the slim tube result of 3100 psig. The final example reported by Zick was the injection of solvent A at the minimum enrichment level (77\% solvent A). Fig. 6.12 shows the key tie line length variations with pressure (for Oil 3 and Gas 3). Again the singular situation arises near 3100 psia. Using the extrapolation technique, we obtained a calculated MMP of 3283 psia, which agrees well with the reported slim tube measurement of 3400 psig. We also performed numerical simulations to calculate the MMPs for these systems. The MMPs are: 2253 psia for Oil 1 and Gas 1; 3093 psia for Oil 2 and Gas 2; and 3256 psia for Oil 3 and Gas 3. All MMPs obtained by numerical simulations agree well with both the analytical MMPs and the slim tube test results. Table 3 and Fig. 6.13 summerize the calculations of MMPs using different methods in comparison with the slim tube measurements of MMPs. Table 3 indicates that the analytical method for calculating MMPs is at least as accurate as the other methods.

\subsection{Discussion}

The MMP calculation technique described here is much faster than conventional compositional simulation. Typical computation times are less than half an hour on a DEC-alpha workstation. Run times for compositional simulation, with attention paid to extrapolation to account for 
effects of numerical dispersion, are at least an order of magnitude greater. Furthermore, the MMP can be determined much more precisely because the calculation can be performed easily for small pressure increments that locate the MMP accurately. Compositional simulations, which are much lengthier, are necessarily restricted to larger pressure increments.

The technique described here can be used in several applications that call for rapid calculation of MMPs. In many gas injection processes, produced gas is reinjected, possibly after blending with gas of some other composition. The tie line intersection method could be used in conjunction with compositional analysis of the injected gas stream to determine whether the blended composition meets a particular MMP specification. Similarly, the method can be used to assess quickly the effect of design injection gas composition changes on MMP, results which will have value as optimum injection gas compositions are determined. In addition, the analytical method could be used to test rapidly whether a proposed fluid characterization gives a calculated MMP consistent with experimental observation. Thus, the proposed method will make possible direct determination of MMPs in situations where previously available calculation techniques were not practical because they were too time consuming.

\subsection{Conclusions}

In this chapter we have proposed an analytical approach to calculate MMPs for displacements involving an arbitrary number of components in either the gas or the oil. The approach is based on the analytical theory of one-dimensional two-phase multicomponent flow. Calculations performed with the tie line intersection technique lead to the following conclusions:

1. Forward and reverse contact calculations with a single mixing cell do not give accurate MMP estimates when development of miscibility is controlled by a tie line other than the tie line that extends through the initial oil (forward contacts) or the injection gas (reverse contacts).

2. MMPs calculated by the tie line intersection technique agree well with experimental observations and with MMPs calculated by compositional simulation. The accuracy of the calculated MMP is determined primarily by the accuracy of the fluid characterization.

3. The MMP calculation technique presented here can be used to test how accurately a fluid characterization reproduces slim tube MMP observations.

\subsection{Nomenclature}

$x=$ liquid phase composition (mole fraction)

$y=$ vapor phase composition (mole fraction)

$V=$ vapor phase mole fraction

$K=$ equilibrium ratio (K-value)

$L=$ tie line length

\subsection{Appendix}

The analytical method for calculating MMPs presented in this chapter relies on the solution of the tie line intersection equations (Eqs. 6.1) for a sequence of increasing pressures. The construction of tie line intersection solutions at a low pressure is a critical first step.

The tie line intersection method requires the solution of a system with $n_{c}\left(2 n_{g}-3\right)-\Sigma_{i=1}^{n_{g}-2} i$ unknowns $\left(n_{c}\right.$ is the total number of components in the system and $n_{g}$ is the number of components 
present in the injection gas). For a realistic phase behavior characterization in which oil and gas are represented by a substantial number of components, the number of unknowns is large. Making a random guess of so many unknowns does not lead to converged solutions, and a more systematic approach is required.

Here we propose a different approach. Instead of trying to make a good initial guess for a particular system at a low pressure, we actually construct the exact solution at the low pressure by starting from a simple system whose solution is easy to construct. The solution for a singlecomponent gas injection system is well established in Johns and Orr[108] and Wang and Orr[220]. We use that approach to find a solution for the actual initial oil but with an injection gas that contains only one component. We found that choosing the component with the highest K-value works well. We then make use of the fact that the solution to a general system is continuous with respect to changes in the pressure and the compositions in oil and gas (Johns et al. [109]), we add a small amount of the component with the second highest K-value to the injection gas and construct the solution for the new system whose injection gas now contains two components. The process of addition of one component continues until the composition of the first component matches that of the actual injection gas. Then we add a small amount of the component with the third highest $\mathrm{K}$-value and construct the solution with three-component injection gas by using the similar solution with a two-component injection gas. The whole process is repeated until the compositions of all components in the gas match those of the actual injection gas. The initial solution is therefore obtained as the exact solution for the actual system we intend to solve at a relatively low pressure far from the MMP.

\subsection{SI Metric Conversion Factors}

psi $\times 6.894757 \mathrm{E}+00=\mathrm{kPa}$

${ }^{\circ} \mathrm{F} \times 0.555556 \mathrm{E}+00={ }^{\circ} \mathrm{K}-255.372$ 


\begin{tabular}{|c||c|c|c|}
\hline \hline \multirow{2}{*}{ Component } & \multicolumn{3}{c|}{ Oil and Gas Compositions (Mole Fraction) (8-comp) } \\
\cline { 2 - 4 } & Reservoir Oil & Lean Gas & Rich Gas \\
\hline \hline $\mathrm{C}_{1}+\mathrm{N}_{2}$ & 0.341 & 0.848 & 0.646 \\
\hline $\mathrm{C}_{2}+\mathrm{CO}_{2}$ & 0.056 & 0.105 & 0.124 \\
\hline $\mathrm{C}_{3}$ & 0.055 & 0.036 & 0.103 \\
\hline $\mathrm{C}_{4}$ & 0.059 & 0.011 & 0.089 \\
\hline $\mathrm{C}_{5}+\mathrm{C}_{6}$ & 0.092 & - & 0.038 \\
\hline $\mathrm{C}_{7+}(1-2)$ & 0.195 & - & - \\
\hline $\mathrm{C}_{7+}(3-4)$ & 0.155 & - & - \\
\hline $\mathrm{C}_{7+}(5)$ & 0.047 & - & - \\
\hline
\end{tabular}

Table 1. Oil and gas compositions (8-component characterization) from Hearn and Whitson ([83]). $\mathrm{T}=212^{\circ} \mathrm{F}$

\begin{tabular}{|c||c|c|c|}
\hline \hline \multirow{2}{*}{ Component } & \multicolumn{3}{c|}{ Oil and Gas Compositions (Mole Fraction) (15-comp) } \\
\cline { 2 - 4 } & Reservoir Oil & Lean Gas & Rich Gas \\
\hline \hline $\mathrm{N}_{2}$ & 0.007 & 0.008 & 0.007 \\
\hline $\mathrm{CO}_{2}$ & 0.006 & 0.015 & 0.013 \\
\hline $\mathrm{C}_{1}$ & 0.334 & 0.840 & 0.639 \\
\hline $\mathrm{C}_{2}$ & 0.050 & 0.091 & 0.111 \\
\hline $\mathrm{C}_{3}$ & 0.055 & 0.036 & 0.103 \\
\hline $\mathrm{IC}_{4}$ & 0.021 & 0.006 & 0.036 \\
\hline $\mathrm{C}_{4}$ & 0.039 & 0.005 & 0.053 \\
\hline $\mathrm{IC}_{5}$ & 0.020 & - & 0.017 \\
\hline $\mathrm{C}_{5}$ & 0.028 & - & 0.014 \\
\hline $\mathrm{C}_{6}$ & 0.044 & - & 0.006 \\
\hline $\mathrm{C}_{7+}(1)$ & 0.096 & - & - \\
\hline $\mathrm{C}_{7+}(2)$ & 0.099 & - & - \\
\hline $\mathrm{C}_{7+}(3)$ & 0.087 & - & - \\
\hline $\mathrm{C}_{7+}(4)$ & 0.068 & - & - \\
\hline $\mathrm{C}_{7+}(5)$ & 0.046 & - & \\
\hline
\end{tabular}

Table 2. Oil and gas compositions (15-component characterization) from Hearn and Whitson $([83]) . \mathrm{T}=212^{\circ} \mathrm{F}$

\begin{tabular}{|c||c|c|c|c|c|c|c|}
\hline \hline \multicolumn{1}{|c||}{ Method } & \multicolumn{7}{c|}{ Minimum Miscibility Pressure (psia) } \\
\cline { 2 - 8 } & Lean(8) & Lean(15) & Rich(8) & Rich(15) & Zick1 & Zick2 & Zick3 \\
\hline \hline Single Cell [20] & 5880 & 5860 & 4520 & 4540 & - & - & - \\
\hline Multiple Cell [20] & - & - & 3205 & 3325 & - & - & - \\
\hline Num. Simul. [20] & 5880 & 5860 & 3205 & 3325 & - & - & - \\
\hline Negative Flash & 6163 & & 6163 & & 7452 & & \\
\hline Analytical & 5582 & 5253 & 3279 & 3115 & 2169 & 3013 & 3283 \\
\hline Slim Tube Test [20][9] & $5215( \pm 200)$ & $5215( \pm 200)$ & $3315( \pm 100)$ & $3315( \pm 100)$ & 2215 & 3115 & 3415 \\
\hline
\end{tabular}

Table 3. Comparison of MMPs calculated by different methods. 


\begin{tabular}{|c||c|c|c|c|c|c|}
\hline \hline \multirow{2}{*}{ Component } & \multicolumn{6}{c|}{ Oil and Gas Compositions (Mole Fraction) } \\
\cline { 2 - 7 } & Oil(Case1) & Gas(Case1) & Oil(Case2) & Gas(Case2) & Oil(Case3) & Gas(Case3) \\
\hline \hline$C O_{2}$ & 0.0492 & 0.2218 & 0.0659 & 0.17744 & 0.06779 & 0.17079 \\
\hline$C H_{4}$ & 0.2164 & 0.2349 & 0.3644 & 0.38792 & 0.38740 & 0.41087 \\
\hline$C_{2}$ & 0.0470 & 0.2350 & 0.0531 & 0.18800 & 0.05352 & 0.18095 \\
\hline$C_{3}$ & 0.0403 & 0.2745 & 0.0373 & 0.21960 & 0.03668 & 0.21136 \\
\hline$C_{4}$ & 0.0318 & 0.0338 & 0.0262 & 0.02704 & 0.02541 & 0.02603 \\
\hline$C_{5}$ & 0.0243 & - & 0.0189 & - & 0.01815 & - \\
\hline$C_{6}-C_{7}$ & 0.0616 & - & 0.0458 & - & 0.04363 & - \\
\hline$C_{8}-C_{10}$ & 0.1196 & - & 0.0879 & - & 0.08339 & - \\
\hline$C_{11}-C_{14}$ & 0.1108 & - & 0.0812 & - & 0.07677 & - \\
\hline$C_{15}-C_{19}$ & 0.0984 & - & 0.0722 & - & 0.06821 & - \\
\hline$C_{20}-C_{29}$ & 0.0908 & - & 0.0666 & - & 0.06297 & - \\
\hline$C_{30+}$ & 0.1098 & - & 0.0805 & - & 0.07608 & - \\
\hline
\end{tabular}

Table 3. Oil and gas compositions from Zick [230]. $\mathrm{T}=185^{\circ} \mathrm{F}$. Case 1 is shown in Fig. 6.10 , Case 2 is shown in Fig. 6.11 and Case 3 is shown in Fig. 6.12. 


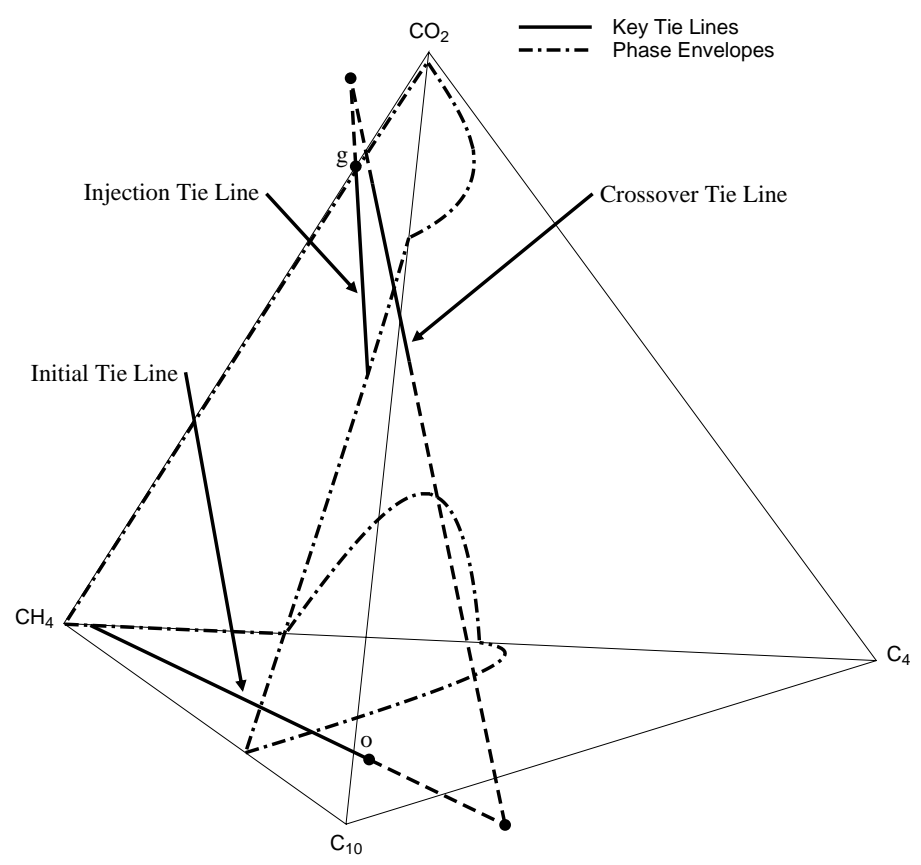

Figure 6.1: Key tie lines intersect each other for a displacement of oil $o$ by gas $g$.

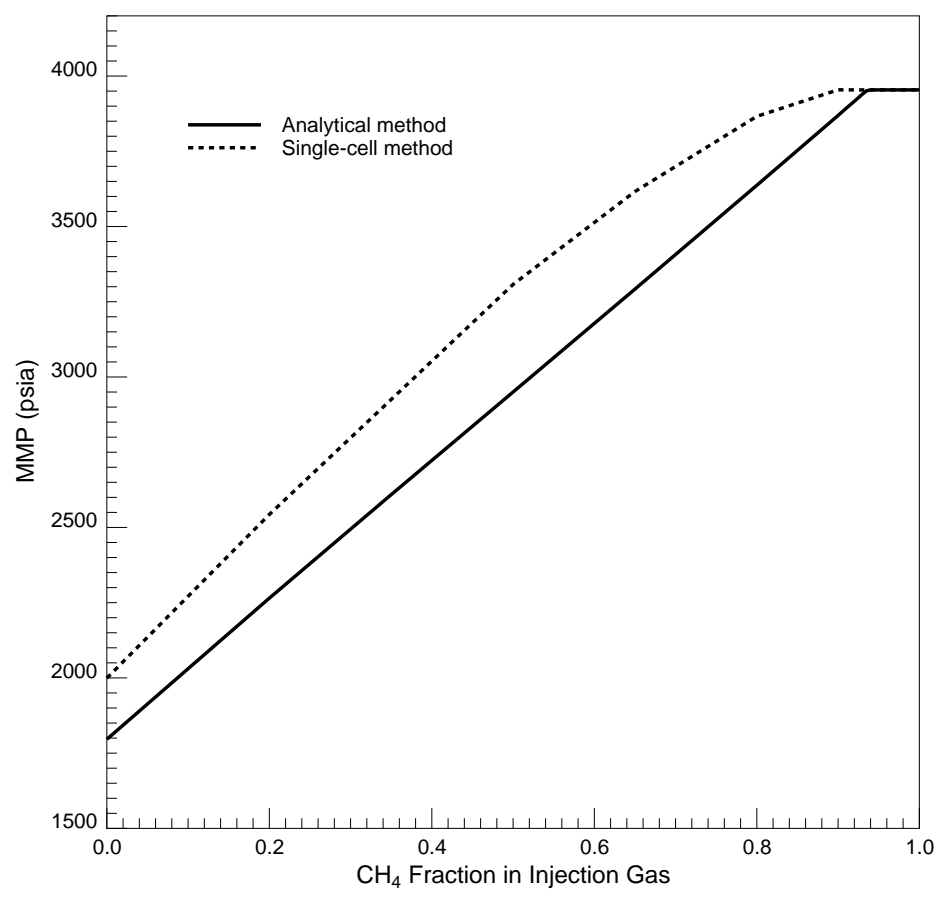

Figure 6.2: Comparison of MMPs calculated by forward contacts in a single mixing cell and by the tie line intersection method. 


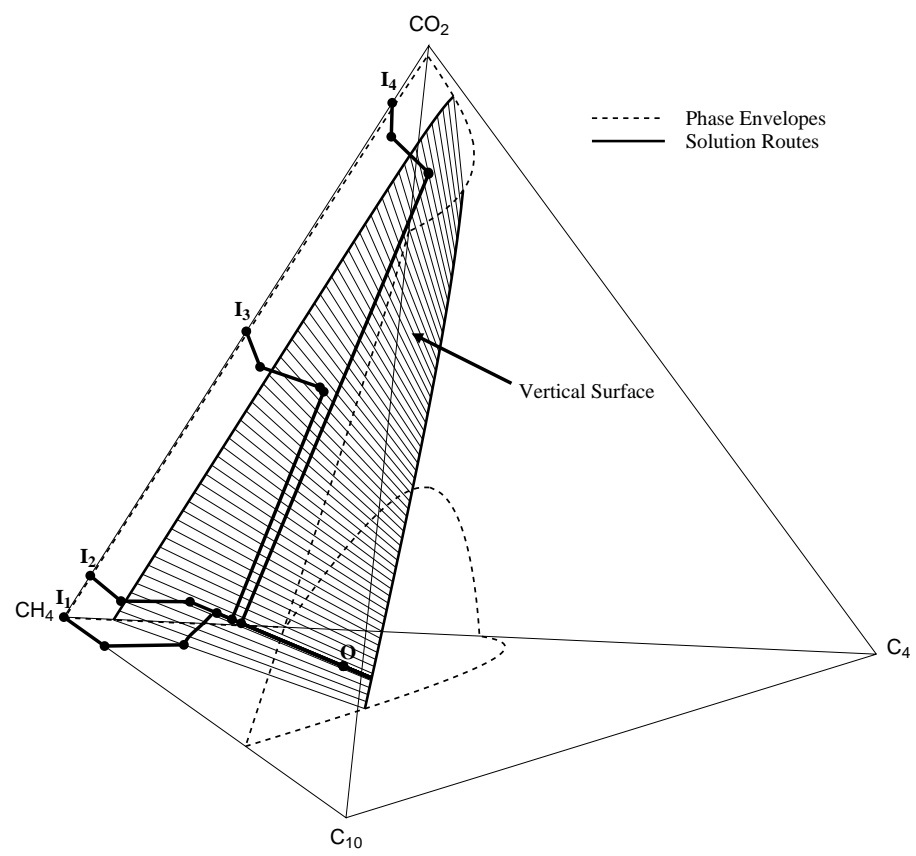

Figure 6.3: Composition paths and crossover tie line surface for the displacement of oil $O$ (40\% $\mathrm{CH}_{4}, 5 \% \mathrm{CO}_{2}, 20 \% \mathrm{C}_{4}$ and $\left.35 \% \mathrm{C}_{10}\right)$ by $I_{1}$ to $I_{4}$ at 1600 psia and $160 \mathrm{~F}$.

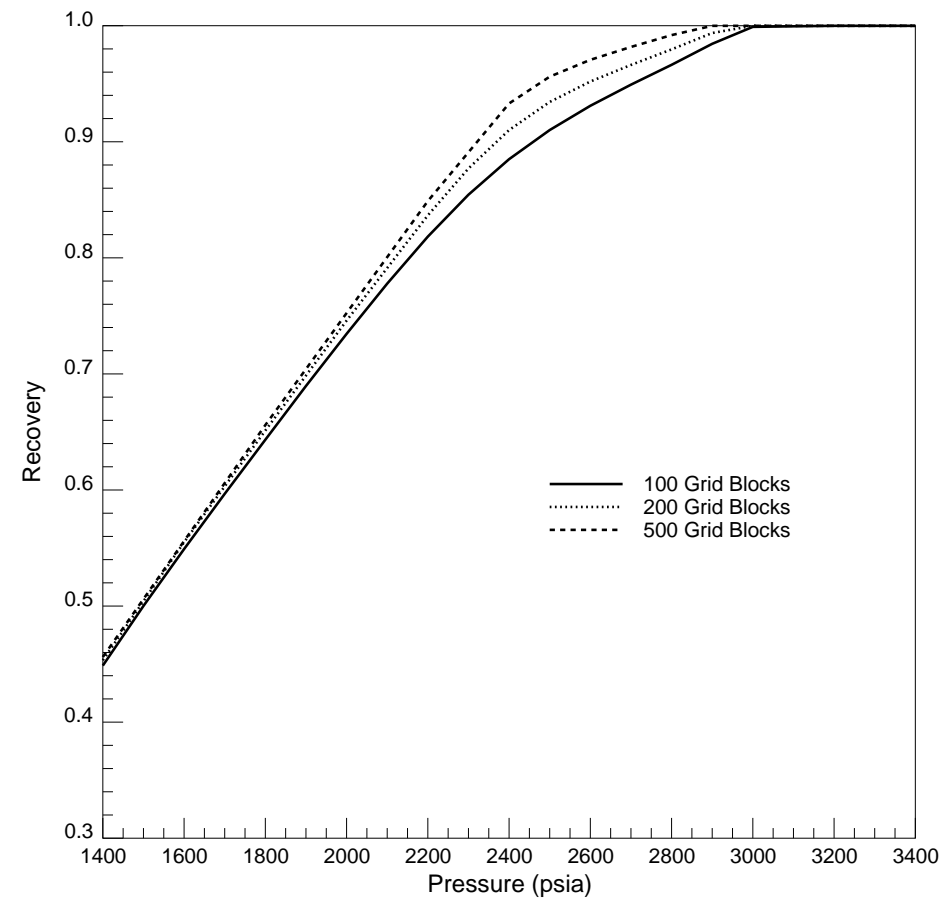

Figure 6.4: Compositional simulation results for the displacement of $40 \% \mathrm{CH}_{4}, 5 \% \mathrm{CO}_{2}, 20 \% \mathrm{C}_{4}$ and $35 \% \mathrm{C}_{10}$ by $50 \% \mathrm{CO}_{2}$ and $50 \% \mathrm{CH}_{4}$ at $160 \mathrm{~F}$. The calculated MMP is 2500 psia. 

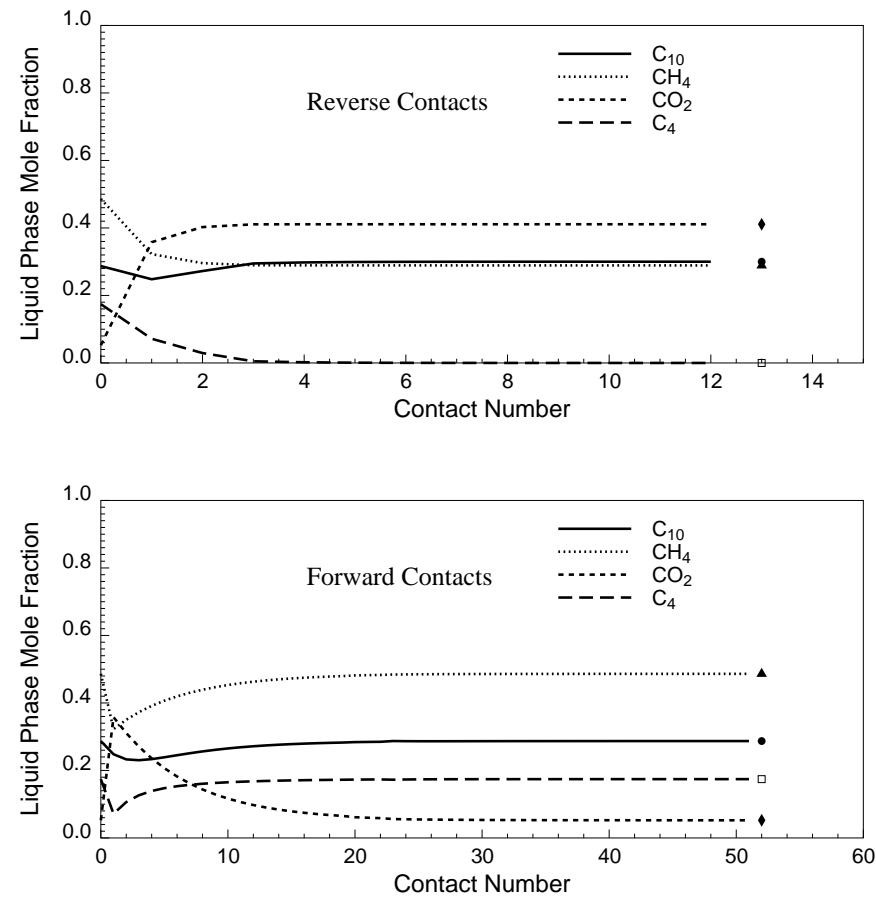

Figure 6.5: Convergence of reverse contacts to the injection gas tie line and forward contacts to the initial oil tie line (symbols indicate the phase compositions obtained by a negative flash).

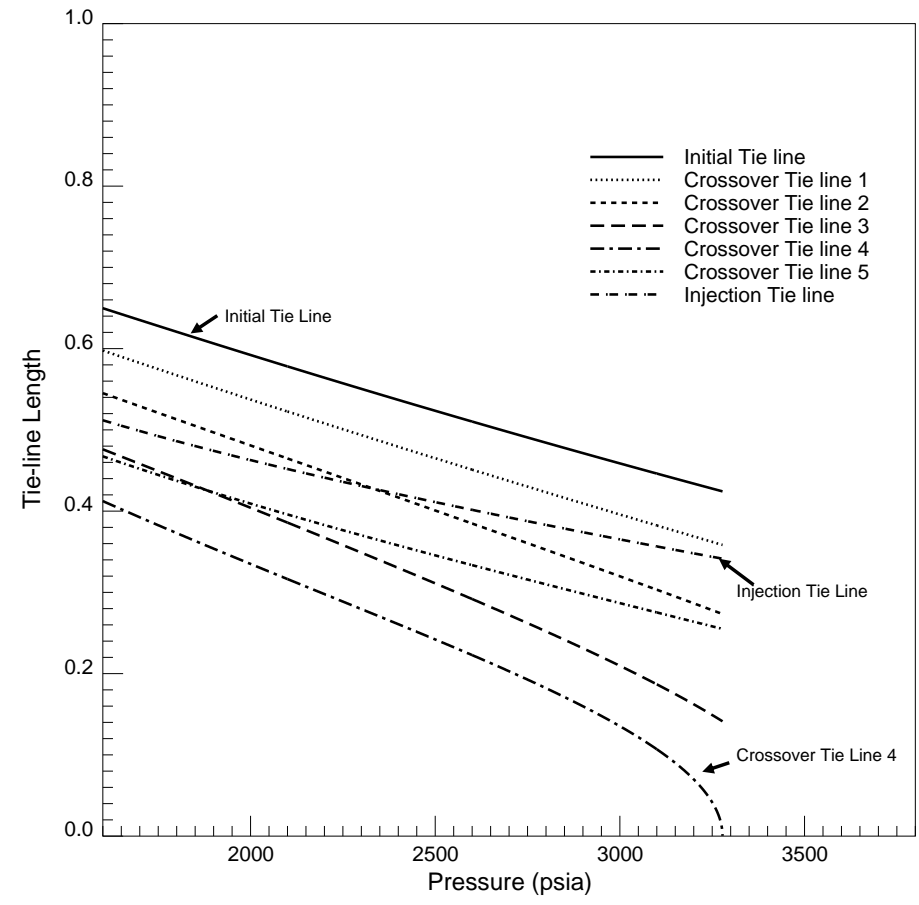

Figure 6.6: Tie line lengths for rich gas displacement of an eight-component oil (Hearn and Whitson [83]) at $212 \mathrm{~F}$. The calculated MMP is 3279 psia. 


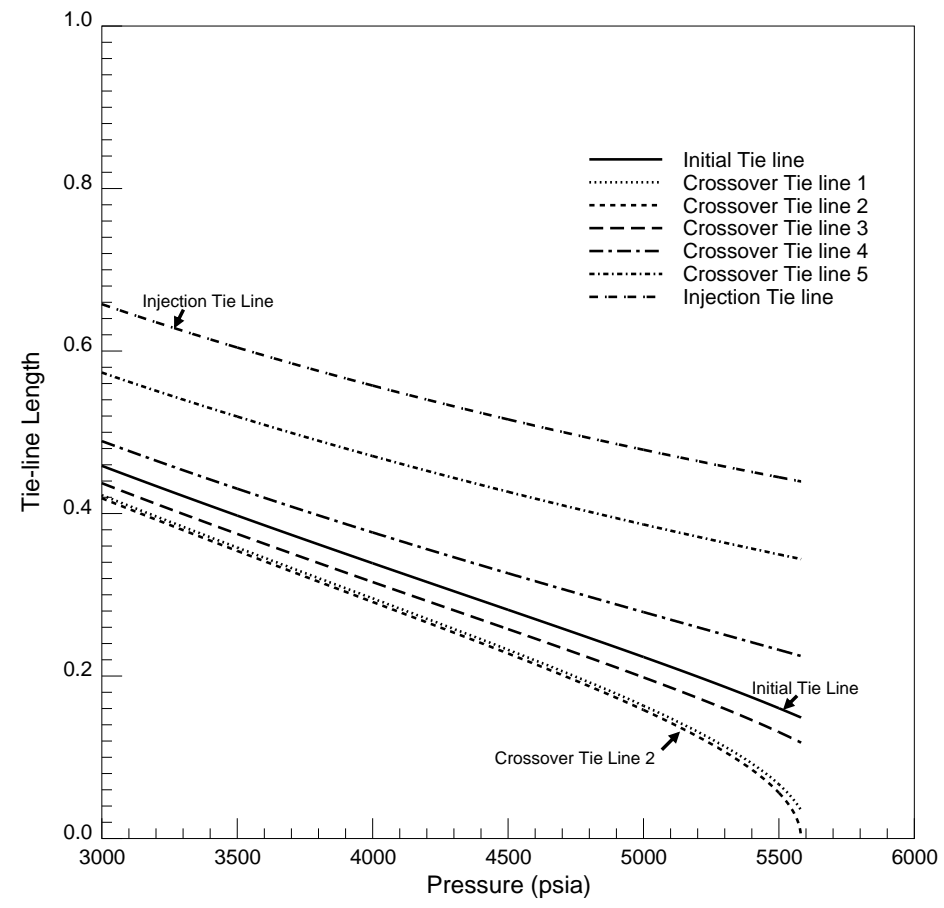

Figure 6.7: Tie line lengths for lean gas displacement of an eight-component oil (Hearn and Whitson [83]) at $212 \mathrm{~F}$. The calculated MMP is 5582 psia.

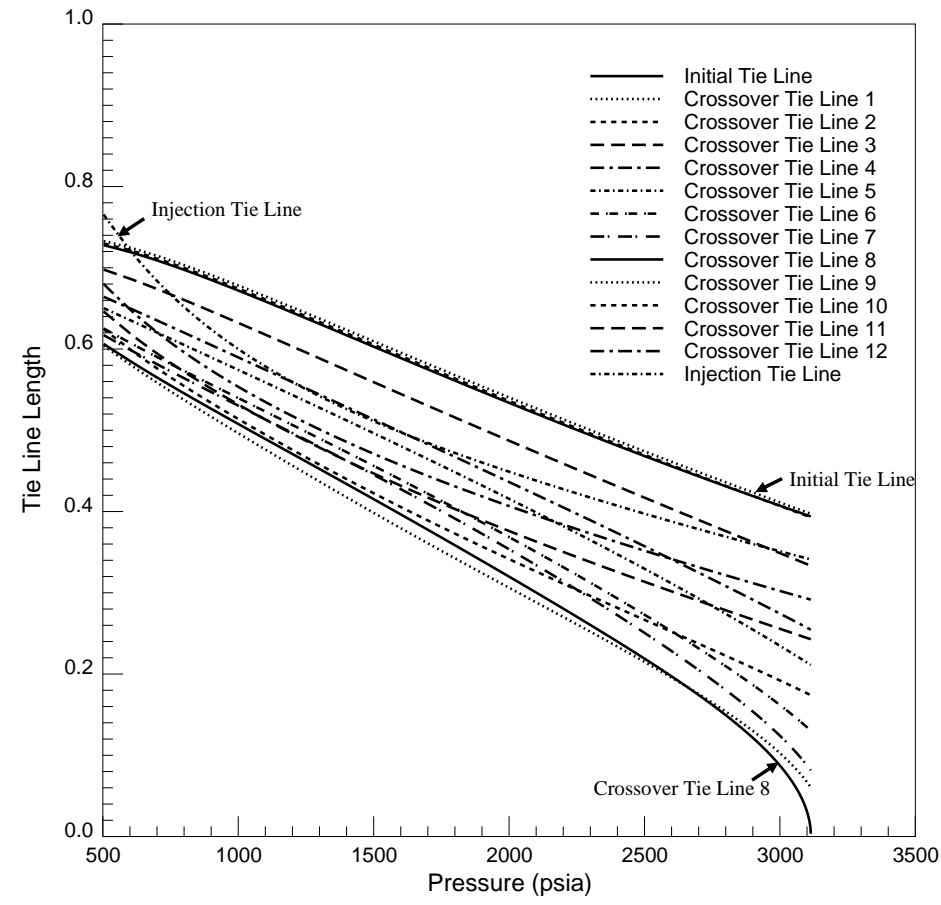

Figure 6.8: Tie line lengths for rich gas displacement of a 15-component oil (Hearn and Whitson [83]) at $212 \mathrm{~F}$. The calculated MMP is 3115 psia. 


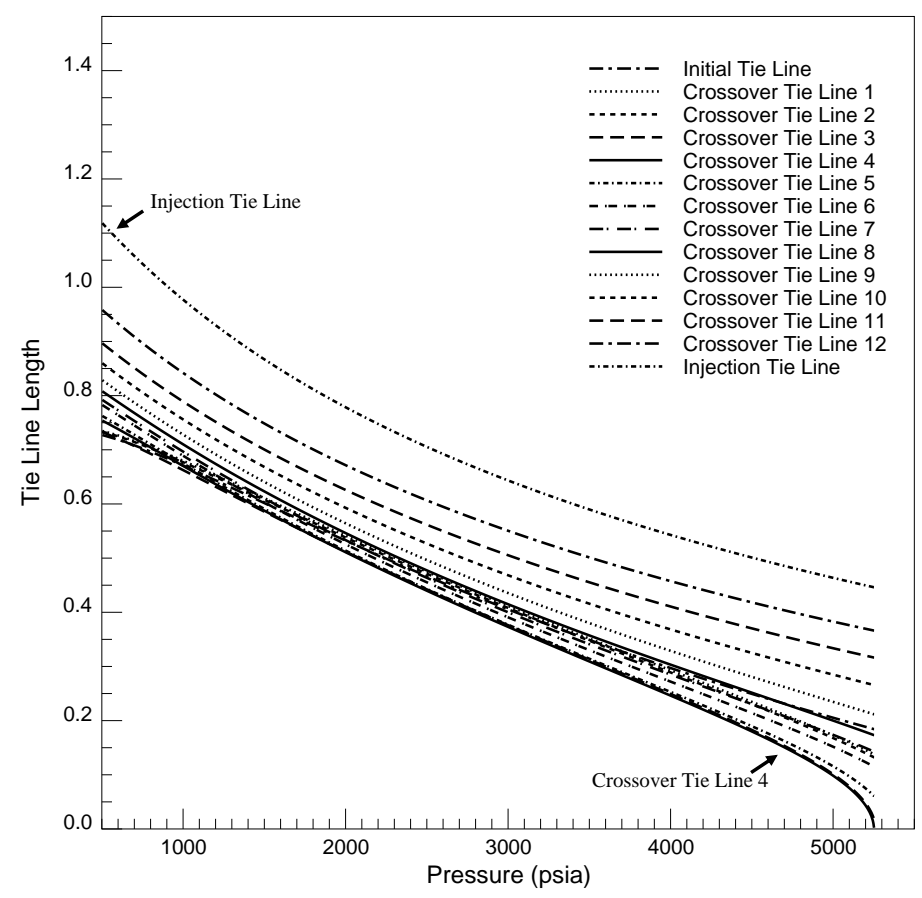

Figure 6.9: Tie line lengths for lean gas displacement of a 15-component oil (Hearn and Whitson [83]) at $212 \mathrm{~F}$. The calculated MMP is 5253 psia.

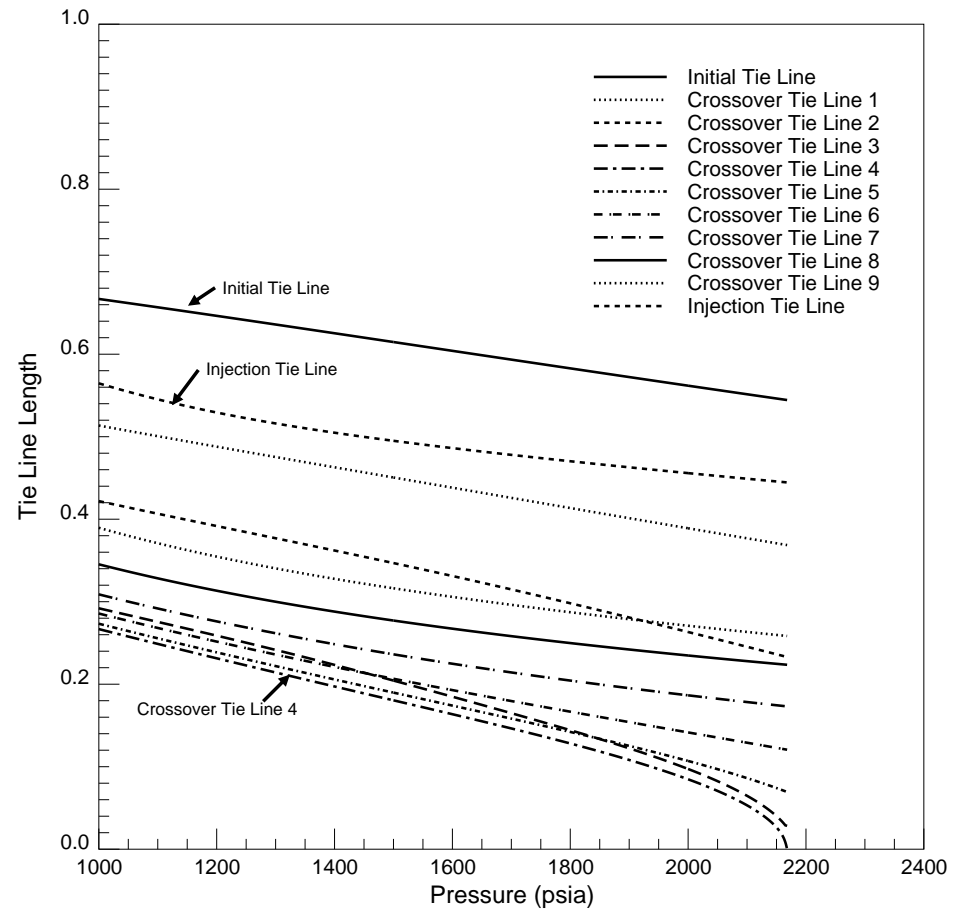

Figure 6.10: Tie line lengths for displacement of oil A (Zick [230]) by solvent A at 185F. The calculated MMP is 2169 psia. 


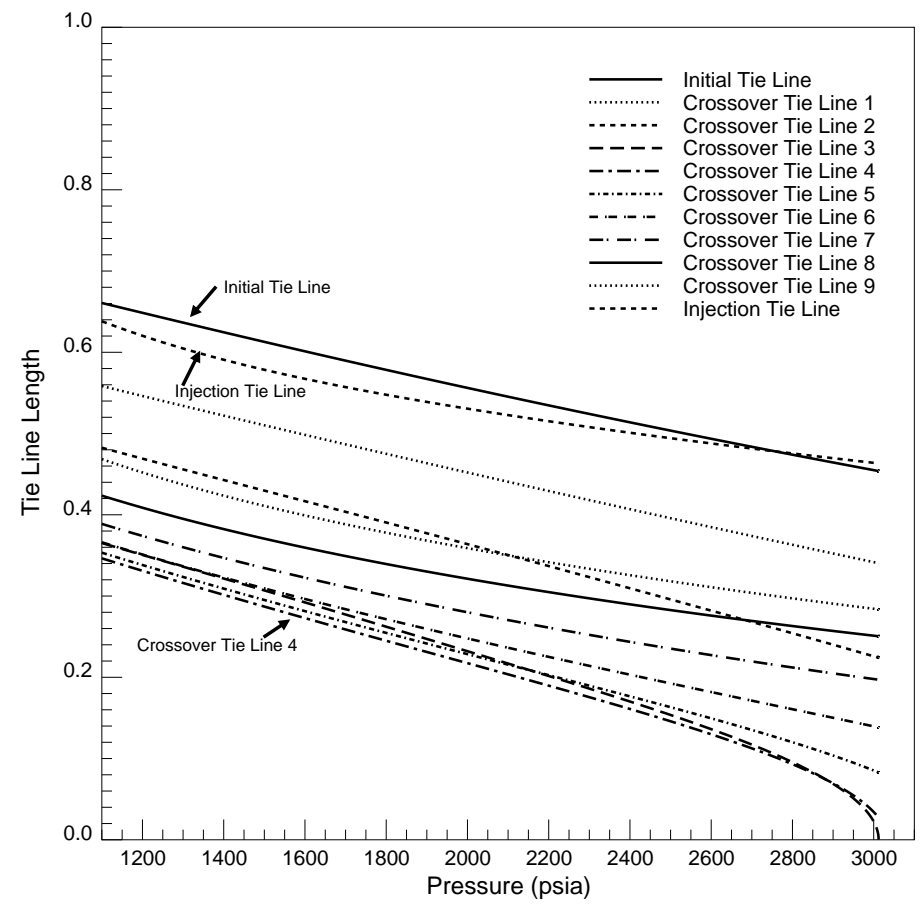

Figure 6.11: Tie line lengths for displacement of oil A (Zick [230]) by $20 \% \mathrm{CH}_{4}$ and $80 \%$ solvent $\mathrm{A}$ at $185 \mathrm{~F}$. The calculated MMP is 3013 psia.

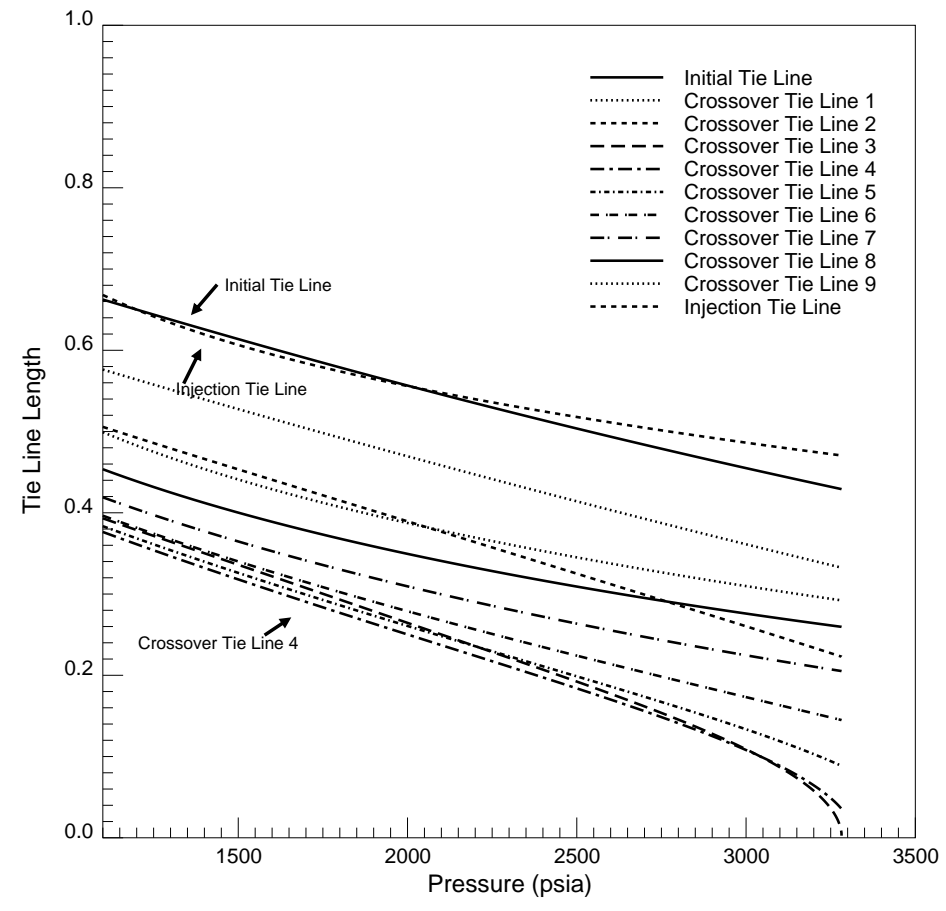

Figure 6.12: Tie line lengths for displacement of oil A (Zick [230]) by $23 \% \mathrm{CH}_{4}$ and $77 \%$ solvent $\mathrm{A}$ at $185 \mathrm{~F}$. The calculated MMP is 3283 psia. 


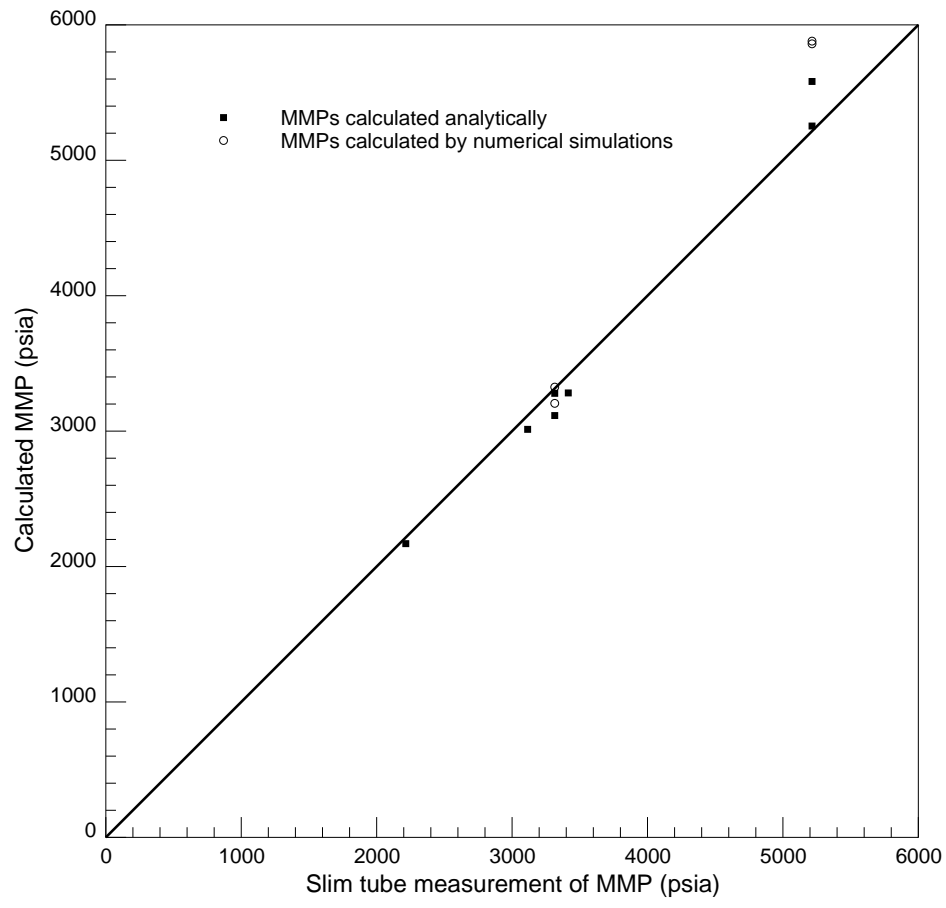

Figure 6.13: Comparison between analytically calculated MMPs with slim tube test results. 


\title{
7. Fast Approximate Solutions for 1D Multicomponent Gas Injection Problems
}

\author{
Kristian Jessen, Yun Wang, Pavel Ermakov, Jichun Zhu, and Franklin M. Orr Jr.
}

This chapter presents a new approach for constructing approximate analytical solutions for onedimensional (1D), multicomponent gas displacement problems. The solution to mass conservation equations governing 1D dispersion-free flow in which components partition between two equilibrium phases is controlled by the geometry of key tie lines. It has previously been proven that for systems with an arbitrary number of components, the key tie lines can be approximated quite accurately by a sequence of intersecting tie lines. As a result, analytical solutions can be constructed efficiently for problems with constant initial and injection compositions (Riemann problems). For fully self-sharpening systems, in which all key tie lines are connected by shocks, the analytical solutions obtained are rigorously accurate, while for systems where some key tie lines are connected by spreading waves, the analytical solutions are approximations, but accurate ones. Detailed comparison between analytical solutions with both coarse and fine grid compositional simulations indicates that even for systems with nontie-line rarefactions, approximate analytical solutions predict composition profiles far more accurately than coarse grid numerical simulations. Because of the generality of the new approach, approximate analytical solutions can be obtained for any system whose phase behavior can be modeled by an equation of state. The construction of approximate analytical solutions is shown to be orders of magnitude faster than the equivalent coarse grid compositional simulation. Hence, the new approach is valuable in areas where fast compositional solutions to Riemann problems are required.

\subsection{Introduction}

Miscible gas injection processes have become a widely used technique for enhanced oil recovery throughout of the world. The understanding of the multiphase, multicomponent flow taking place in any miscible displacement process is essential for successful design of gas injection projects. Due to complex reservoir geometry and reservoir fluid properties, numerical simulations of the flow processes are usually conducted to obtain such understanding. In principle, compositional simulation could be used to study such problems. In practice, however, conventional finite difference simulation is sufficiently slow that three-dimensional (3D) computations are feasible only for very coarse grids. Such simulations are not useful, however, because they are severely affected by numerical dispersion.

Recent progress in the application of streamline methods offers one way to overcome the limitations of 3D finite difference compositional simulations[205, 18, 10]. In the streamline approach, a one-dimensional (1D) solution is mapped onto streamlines that capture the effects of reservoir heterogeneity. Chapter 3[204] described 2D and 3D streamline compositional simulations in which analytical and finite difference approaches were used to solve the 1D flow problem. In CHapter 3 a numerical solution was used for the 1D problem to perform a compositional simulation for a heterogeneous 3D reservoir described with 518,000 grid blocks. At the time that work was done, analytical solutions for problems with an arbitrary number of components in the oil and injection gas were not available. In this chapter, we describe an algorithm to obtain analytical solutions for

\footnotetext{
${ }^{6}$ The material in this chapter was presented at the 1999 SPE Annual Technical Conference and Exhibition in Houston, TX, Oct 3-6, 1999, and is published as SPE paper 56608 in the conference proceedings.
} 
that problem. Use of the analytical solutions in simulations like those of Thiele et al.[204] could lead to substantial additional speed-ups in streamline calculations.

A substantial body of mathematical theory now exists for construction of analytical solutions to the dispersion-free 1D multicomponent flow problem[143, 109, 110, 108, 45, 43]. This theory, based on the method of characteristics (MOC), illustrates that the behavior of the solution is controlled by the geometry of key tie lines in compositional space. Wang and Orr[220, 221] and subsequently Jessen et al.[106] have developed algorithms for approximating the key tie lines. In this chapter the key tie line approximation is combined with tools from the analytical theory of gas displacement to obtain fast, approximate solutions to the 1D, dispersion-free two phase flow problem.

\subsection{Analytical Theory of 1D Miscible Displacements}

The analytical theory of gas injection processes describes the complex interactions between two-phase flow and phase equilibrium for 1D dispersion-free miscible displacements. Analytical solutions obtained in this chapter are based on the following assumptions:

- The porous medium is homogeneous.

- Instant thermodynamic equilibrium exists everywhere.

- No gravity or capillary forces act on the fluid.

- Pressure and temperature are constant throughout the porous medium.

- Components do not change volume as they transfer between phases.

The assumption of no volume change is reasonable when pressures are high. For systems at lower pressures where solubility of light components in undisplaced oil is high but gas density is low, effects of volume change can be significant[43] and Dindoruk's formulation of the conservation equations should be used.

Based on these assumptions, the mass conservation equations are written in the form

$$
\frac{\partial C_{i}}{\partial t}+\frac{\partial F_{i}}{\partial z}=0, i=1, \ldots, n_{c}
$$

where $C_{i}$ is the overall volumetric fraction and $F_{i}$ is the overall fractional flow of component $i, t$ is dimensionless time, $z$ is dimensionless distance and $n_{c}$ is the number of components in the mixture. Considering two-phased flow, $C_{i}$ and $F_{i}$ are connected to the phase behavior by

$$
\begin{gathered}
C_{i}=c_{i l}(1-S)+c_{i v} S, \\
F_{i}=c_{i l}(1-f)+c_{i v} f .
\end{gathered}
$$

$S$ is the volumetric vapor fraction and $f$ is the fractional flow of vapor, whereas $c_{i l}$ and $c_{i v}$ are the volume fractions of component $i$ in the liquid and vapor phases respectively. $C_{i}$ and $F_{i}$ are subject to the constraint

$$
\sum_{i=1}^{n_{c}} C_{i}=\sum_{i=1}^{n_{c}} F_{i}=1
$$

The fractional flow function used for this work is

$$
f=\frac{S^{2}}{S^{2}+M\left(1-S-S_{o r}\right)^{2}},
$$


where $M$ is the viscosity ratio (vapor/liquid), and $S_{\text {or }}$ is the residual oil saturation. Finally the initial data are specified by

$$
C(z, 0)=\left\{\begin{array}{ll}
C_{i}^{i n j} & z<0 \\
C_{i}^{i n i} & z>0
\end{array} i=1, \ldots, n_{c} .\right.
$$

The specification of constant initial and injection compositions to the mass conservation equations make the construction of analytical solutions to the flow problem possible. Eqs. 7.1-7.6 specify a Riemann problem.

Analytical solutions to Eqs. 7.1-7.6 are constructed by solving the eigenvalue problem associated with the mass conservation equations. In composition space, the corresponding problem is to identify the correct (unique) route that connects the initial oil composition and the injection gas composition. The composition route that describes the analytical solution geometrically is subject to the following requirements.

The composition route must have characteristic wave velocities in the two-phase region that increase monotonically from upstream to downstream locations. This condition is known as the velocity rule. If the velocity rule would be violated by a continuous variation (rarefaction), then a shock must be introduced to insure that the solution remains single-valued. The shock must satisfy the integral form of the mass conservation equations.

$$
\Lambda=\frac{F_{i}^{u}-F_{i}^{d}}{C_{i}^{u}-C_{i}^{d}}, i=1, \ldots, n_{c}
$$

where $\Lambda$ is the shock velocity. Upstream and downstream parts of the shock are denoted $\mathrm{u}$ and $\mathrm{d}$ respectively. Eq. 7.7 is a Rankine-Hugoniot condition. Any shock present in a solution must satisfy an entropy condition, which requires the shock to be stable in the presence of a small amount of dispersion. In addition, solutions must satisfy a continuity condition with respect to initial and injection data. In other words, small perturbations to the initial or injection compositions must result in small changes in the solution.

For all the examples presented in this chapter the Soave-Redlich-Kwong equation of state was used for phase equilibrium calculation, whereas the Lohrenz-Bray-Clark[128] correlation was used for calculation of viscosity.

\subsection{Self-Sharpening Systems}

Fully self-sharpening systems are characterized by the feature that all key tie lines are connected by shocks. For such systems the key tie line intersection approach is rigorously accurate because the extension of a pair of key tie lines connected by a shock must intersect[109, 110, 108, 45, 43]. Two types of shocks occur. When the shock velocity matches the tie-line eigenvalue $(d f / d S)$ on one side of the shock, the shock is known as a tangent shock. When the shock velocity differs from the wave velocities on both sides of the shock, the shock is called a genuine shock. Both types of shocks occur in typical solutions. Because the tie lines that make up the solution can be found by the intersecting tie line approach[220, 221, 106] a full solution can be constructed if the shock composition points can be determined on each of the key tie lines. The only remaining question is: On which tie line does solution construction begin. We will refer to that tie line as the "primary" tie line.

Solution construction begins with finding the tangent shocks that connect the primary tie line to adjacent tie lines just upstream and downstream. For problems in which the injection gas composition lies on the vapor side of the two-phase region, the composition path lies on the vapor side of the equivelocity curve (where $f=S$ ) [85]. For such compositions, $f>S$. 

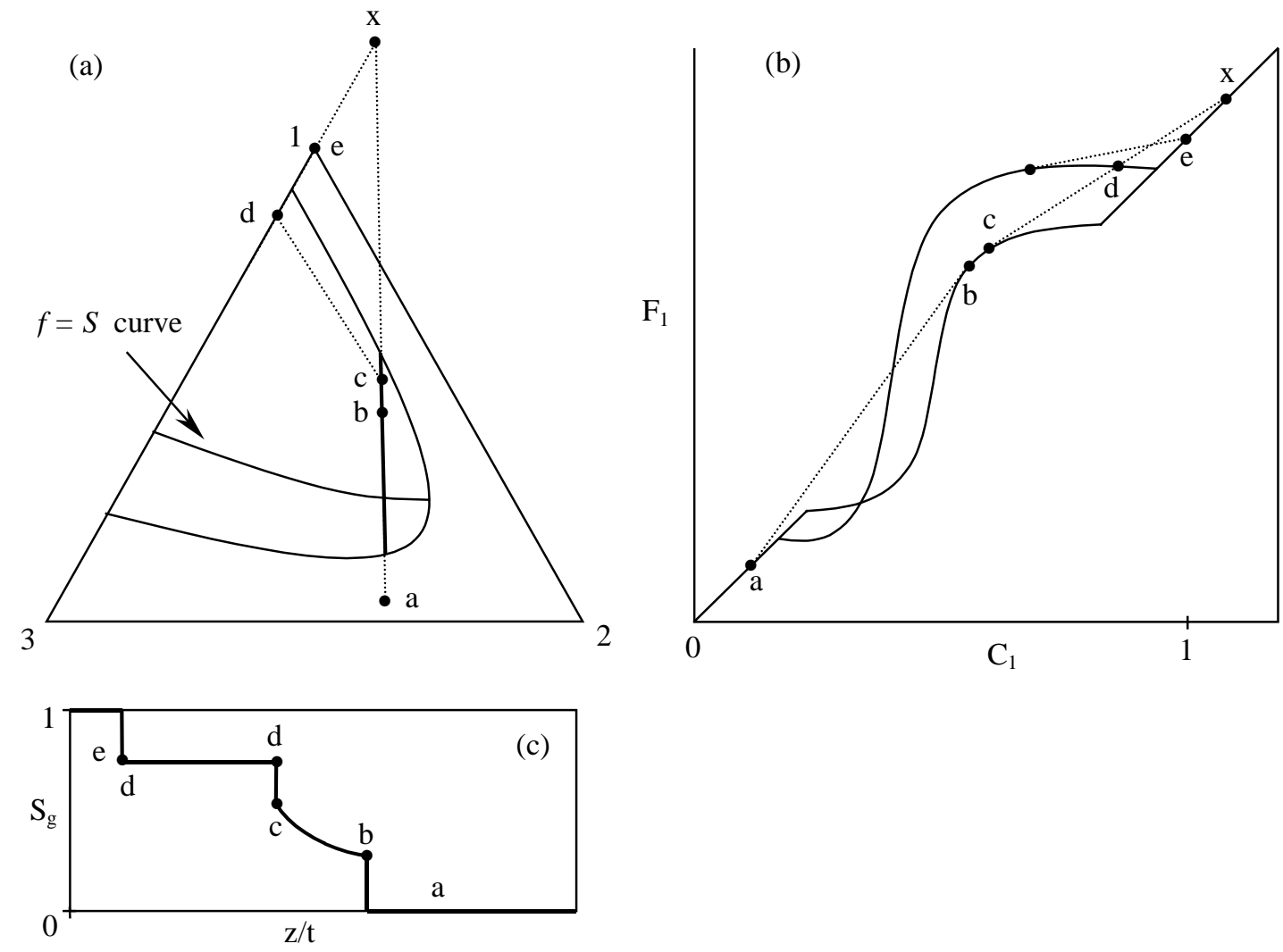

Figure 7.1: Construction of tangent shocks.

We show now that the primary tie line must be the shortest of the key tie lines. To see why this statement must be true, we consider a simple ternary vaporizing gas drive (Fig. 7.1a) in which oil (composition a) is displaced by gas (composition e). Two key tie lines make up the solution: the tie line that extends through the initial oil composition, and the tie line that extends through the gas composition. In this example, the tie lines are connected by a shock because a rarefaction between the oil tie line and the gas tie line would violate the velocity rule. The corresponding overall fractional flow curves for the two key tie lines are shown in Fig. 7.1b, and the saturation profile for the solution is shown in Fig. 7.1c. In this case, the leading shock is a tangent shock, found by constructing the chord from point a in Fig. 7.1b that is tangent to the fractional flow curve for the oil tie line. The shock from point $c$ on the oil tie line is found by constructing a chord from point $\mathrm{X}$ that is tangent to the fractional flow curve for the oil tie line. Point $\mathrm{X}$ (Fig. 7.1a) is the intersection point of the two key tie lines. Point $\mathrm{X}$ in Fig. 7.1b lies on the $F 1=C 1$ line. The composition of point $\mathrm{d}$, the landing point on the gas tie is given by the intersection of the chord with the fractional flow curve of the gas tie line. Note that point $d$ lies above the composition at which a chord constructed from the gas composition, point e, would be tangent to the fractional flow curve for the gas tie line. A continuous variation from point $d$ to that tangent point would violate the velocity rule, so a genuine shock from point $\mathrm{d}$ to point e is required. The velocity of that shock is given by the slope of the chord from point $d$ to point $e$.

The tangent drawn from point $\mathrm{X}$ to point $\mathrm{c}$ in Fig. 7.1b satisfies the following equations[109]:

$$
\frac{F_{i}^{c}-F_{i}^{d}}{C_{i}^{c}-C_{i}^{d}}=\frac{F_{i}^{c}-C_{i}^{x}}{C_{i}^{c}-C_{i}^{x}}=\frac{F_{i}^{d}-C_{i}^{x}}{C_{i}^{d}-C_{i}^{x}}=\frac{f^{c}-S^{c x}}{S^{c}-S^{c x}}=\frac{S^{d}-S^{d x}}{S^{d}-S^{d x}}=\left.\frac{d f}{d S}\right|_{c},
$$




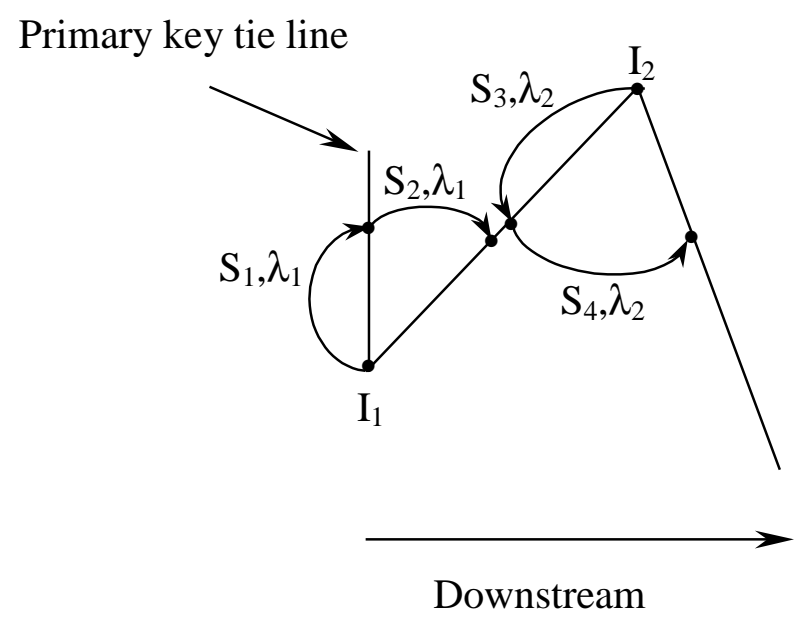

Figure 7.2: Illustration of downstream solution path construction.

where $S^{c x}$ and $S^{d x}$ refer to the saturations at point X measured on the tie lines containing points $c$ and d:

$$
S^{c x}=\frac{C_{1}^{x}-c_{1 l}^{c}}{c_{1 v}^{c}-c_{1 l}^{c}}, S^{d x}=\frac{C_{1}^{x}-c_{1 l}^{d}}{c_{1 v}^{d}-c_{1 l}^{d}} .
$$

Fig. 7.1b shows that the tangent constructed from point $\mathrm{c}$ to $\mathrm{X}$ intersects the overall fractional flow curve for the injection gas tie line at point $d$. If, on the other hand, the chord had been drawn from point $\mathrm{X}$ to the tangent point on the gas tie line, the extension of the chord would not intersect the overall fractional flow curve for the oil tie line. Thus it is not possible to satisfy the shock equations if the tangent were constructed to the gas tie line, but it is possible to do so for the oil tie line. Analysis of the shock equations indicates that it is always possible to satisfy the shock equations for this example if $S^{c x}>S^{d x}$. That restriction is controlled by the length of the tie lines. In this example, the oil tie line is short, and the gas tie line is longer, so $c_{1 v}^{c}-c_{1 l}^{c}<c_{1 v}^{d}-c_{1 l}^{d}$. Because the differences in $c_{1 l}^{c}$ and $c_{1 l}^{d}$ are small, the length of the tie lines dominates the saturations in Eq. 7.9. Thus, if a shock between two tie lines is a tangent shock, the tangent must be constructed to the shorter of the two tie lines.

Similar reasoning can be applied sequentially to each adjacent pair of tie lines, with the result that in fully self-sharpening systems the shortest of the $n_{c}-1$ key tie lines must be a tie line that is connected to tie lines just upstream and downstream by tangent shocks. The tangents constructed from the intersection points to the shortest tie line. Therefore, the shortest tie line is the primary tie line.

The algorithm for construction of fully self-sharpening 1D solutions is:

1. Locate all key tie lines using the tie line intersection approach. The global solution algorithm of Jessen et al.[106] was used here.

2. Locate the primary (shortest) key tie line and start tracing the solution upstream and downstream. For each adjacent pair of tie lines, the possibility of a tangent construction is initially investigated. The construction procedure is illustrated in Fig. 7.2.

3. Downstream construction: A tangent construction is made by solving Eq. 7.8 for $\left(S_{1}, \lambda_{1}\right)$ on the primary tie line from the first downstream intersection point $\left(\mathrm{I}_{1}\right)$. The landing point 
on the down-stream tie line $\left(S_{2}, \lambda_{1}\right)$ is subsequently found by solving Eq. 7.8 with the shock velocity fixed.

4. For the next downstream pair, intersecting at $\mathrm{I}_{2}$, a new tangent construction is examined. From this point and forward the tangent construction may be invalid due to a violation of the velocity rule. That is, if the endpoint of the previous construction $\left(S_{2}, \lambda_{1}\right)$ has a velocity that is higher than the jump or landing point from new shock construction $\left(S_{3}, \lambda_{2}\right)$. In such a case, the new pair of tie lines is connected by a genuine shock. A genuine shock is followed by a constant state. If, on the other hand, two tangent constructions are made to the same tie line the tangent points are connected by either a direct jump or by continuous variation along the tie line.

5. Step 4 is repeated until the tie line extending through the initial oil is reached. The solution path often enters (and leaves) the two-phase region by a tangent shock. For some systems, (Fig. 7.1, for example), however, variation along the tie line violates the velocity rule and a direct jump is used.

6. From the primary tie line, the solution path is traced upstream by the approach of steps 3 and 4.

\subsection{Solution Example for Fully Self-Sharpening System}

The algorithm for construction of self-sharpening solutions has been applied for a real reservoir fluid. The reservoir fluid was characterized by the procedure of Pedersen et al.[174] into a 15 component fluid description. The properties of the characterized fluid are given in Table 7.1. The reservoir temperature is $387.45 \mathrm{~K}$ at which the bubble point pressure of the original oil (A) is 252 atm. The pure component critical volumes $V_{c}$, used for the prediction of phase viscosities, were calculated by specifying the critical compressibility factor of all components to 0.307 . We seek the 1D solution for the displacement of oil A by gas A at 275 atm.

The fourteen key tie lines (those extending through the oil and gas compositions and twelve crossover tie lines) were determined[106], and the third crossover tie line was identified as the primary (shortest) tie line, the starting point for shock construction. The saturation profile is shown in Fig. 7.3, and the details of the solution are given in Table 7.2. The primary tie line is connected to the first downstream tie line by a tangent shock (d1). The remaining downstream part of the solution consists of genuine shocks, constant states, and a direct jump from the oil tie line to the initial oil composition. The upstream part of the solution starts with a tangent shock (d2) connecting the primary tie line to the next crossover tie line. The remaining upstream shocks are all genuine shocks with associated constant states, and another genuine shock connects the injection gas tie line with the injection gas composition. A continuous variation connects the two shock points ( $\mathrm{d} 1$ and $\mathrm{d} 2$ ) on the primary tie line.

In order to confirm the analytical solution, a series of finite difference (FD) simulations was performed. Single-point upstream weighting with a Courant number $(\Delta z / \Delta t)$ of 10 was used in all the simulations which were run on a $450 \mathrm{MHz}$ PC. The new two-phase PT-flash algorithm developed by Michelsen[140] was used in the FD simulator to speed up the numerical solutions. The numerical saturation profiles from simulations using 100, 1000 and 10000 grid blocks are compared with the analytical profile in Fig. 7.3. The CPU time required to construct the analytical solution was 0.9 second, compared to 4.4 seconds, 5.4 minutes and 7.8 hours used respectively by the numerical simulations. The coarse grid simulation (100 grid blocks) is not able to describe the details but only the general trend of the dispersion-free solution. More details are captured using 1000 grid blocks and an excellent agreement is observed when using 10000 grid blocks. However, the CPU 


\begin{tabular}{||c|c|c|c|c|c|c||}
\hline \hline Comp. & $\mathrm{T}_{\mathrm{c}}(\mathrm{K})$ & $\mathrm{P}_{\mathrm{c}}(\mathrm{atm})$ & $\omega$ & $\mathrm{M}_{\mathrm{w}}(\mathrm{g} / \mathrm{mole})$ & x-Oil A & $\mathrm{y}$-Gas A \\
\hline $\mathrm{N}_{2}$ & 126.200 & 33.6000 & 0.0400 & 28.016 & 0.450 & 0.49 \\
\hline $\mathrm{CO}_{2}$ & 304.200 & 72.9000 & 0.2280 & 44.010 & 1.640 & 1.82 \\
\hline $\mathrm{CH}_{4}$ & 190.600 & 45.4000 & 0.0080 & 16.043 & 45.850 & 81.39 \\
\hline $\mathrm{C}_{2}$ & 305.400 & 48.2000 & 0.0980 & 30.069 & 7.150 & 9.15 \\
\hline $\mathrm{C}_{3}$ & 369.800 & 41.9000 & 0.1520 & 44.096 & 6.740 & 4.67 \\
\hline $\mathrm{i}-\mathrm{C}_{4}$ & 408.100 & 36.0000 & 0.1760 & 58.123 & 0.840 & 0.50 \\
\hline $\mathrm{n}-\mathrm{C}_{4}$ & 425.200 & 37.5000 & 0.1930 & 58.123 & 3.110 & 1.24 \\
\hline $\mathrm{i}-\mathrm{C}_{5}$ & 460.400 & 33.4000 & 0.2270 & 72.150 & 1.030 & 0.20 \\
\hline $\mathrm{n}-\mathrm{C}_{5}$ & 469.600 & 33.3000 & 0.2510 & 72.150 & 1.650 & 0.26 \\
\hline $\mathrm{C}_{6}$ & 507.400 & 29.3000 & 0.2960 & 86.177 & 2.520 & 0.09 \\
\hline $\mathrm{C}_{7}$ & 632.800 & 30.2987 & 0.1842 & 109.007 & 12.440 & 0.19 \\
\hline $\mathrm{C}_{11}$ & 659.605 & 23.4598 & 0.4773 & 175.327 & 6.320 & 0.00 \\
\hline $\mathrm{C}_{16}$ & 703.646 & 19.2900 & 0.8197 & 256.674 & 5.024 & 0.00 \\
\hline $\mathrm{C}_{23}$ & 766.497 & 16.7852 & 1.2114 & 370.099 & 3.240 & 0.00 \\
\hline $\mathrm{C}_{33}$ & 892.990 & 15.1302 & 1.3718 & 590.374 & 1.996 & 0.00 \\
\hline
\end{tabular}

Table 7.1: Characterized fluid description (SRK - Equation of state).

\begin{tabular}{||c|c|c|c|c|c|c||}
\hline Point & Tie line & $\mu_{\text {gas }} / \mu_{\text {oil }}$ & $\log \left(\mathrm{K}_{\mathrm{nc}}\right)$ & $\mathrm{Z}_{\mathrm{nc}}$ & $\mathrm{S}$ & $\lambda$ \\
\hline $\mathrm{Oil}$ & Initial & 0.1719 & -5.80352 & 0.019957 & - & $1.9972-\infty$ \\
\hline $\mathrm{a}$ & Initial & 0.1719 & -5.80352 & 0.011376 & 0.321 & $1.4501-1.9972$ \\
\hline $\mathrm{b}$ & 1 & 0.1758 & -5.70171 & 0.010801 & 0.344 & $1.2170-1.4501$ \\
\hline $\mathrm{c}$ & 2 & 0.1740 & -5.73387 & 0.010972 & 0.338 & $1.1270-1.2170$ \\
\hline $\mathrm{d}_{1}$ & 3 & 0.1879 & -5.46043 & 0.008974 & 0.424 & 1.1270 \\
\hline $\mathrm{d}_{2}$ & 3 & 0.1879 & -5.46043 & 0.008732 & 0.438 & 1.0187 \\
\hline $\mathrm{e}$ & 4 & 0.1782 & -5.64053 & 0.007761 & 0.509 & $0.9505-1.0187$ \\
\hline $\mathrm{f}$ & 5 & 0.1774 & -5.65381 & 0.007727 & 0.512 & $0.9086-0.9505$ \\
\hline $\mathrm{g}$ & 6 & 0.1674 & -5.83705 & 0.007376 & 0.547 & $0.8407-0.9086$ \\
\hline $\mathrm{h}$ & 7 & 0.1608 & -5.95911 & 0.007205 & 0.567 & $0.8155-0.8407$ \\
\hline $\mathrm{i}$ & 8 & 0.1500 & -6.16425 & 0.007001 & 0.595 & $0.7360-0.8155$ \\
\hline $\mathrm{j}$ & 9 & 0.1281 & -6.60241 & 0.006702 & 0.646 & $0.4823-0.7360$ \\
\hline $\mathrm{k}$ & 10 & 0.0705 & -7.72998 & 0.006246 & 0.780 & $0.3521-0.4823$ \\
\hline $\mathrm{I}$ & 11 & 0.0391 & -8.54209 & 0.006109 & 0.862 & $0.1630-0.3521$ \\
\hline $\mathrm{m}$ & 12 & 0.0219 & -9.01158 & 0.006151 & 0.927 & $0.0351-0.1630$ \\
\hline $\mathrm{n}$ & Inj. & 0.0150 & -9.12906 & 0.008519 & 0.960 & $0.0163-0.0351$ \\
\hline $\mathrm{o}$ & Inj. & 0.0150 & -9.12906 & 0.000049 & 1 & $0-0.0163$ \\
\hline $\mathrm{Gas}$ & Inj. & 0.0150 & -9.12906 & 0.000000 & - & 0 \\
\hline
\end{tabular}

Table 7.2: MOC solution for displacement of Oil A by Gas A at $275 \mathrm{~atm}$ and $387 \mathrm{~K}$. 


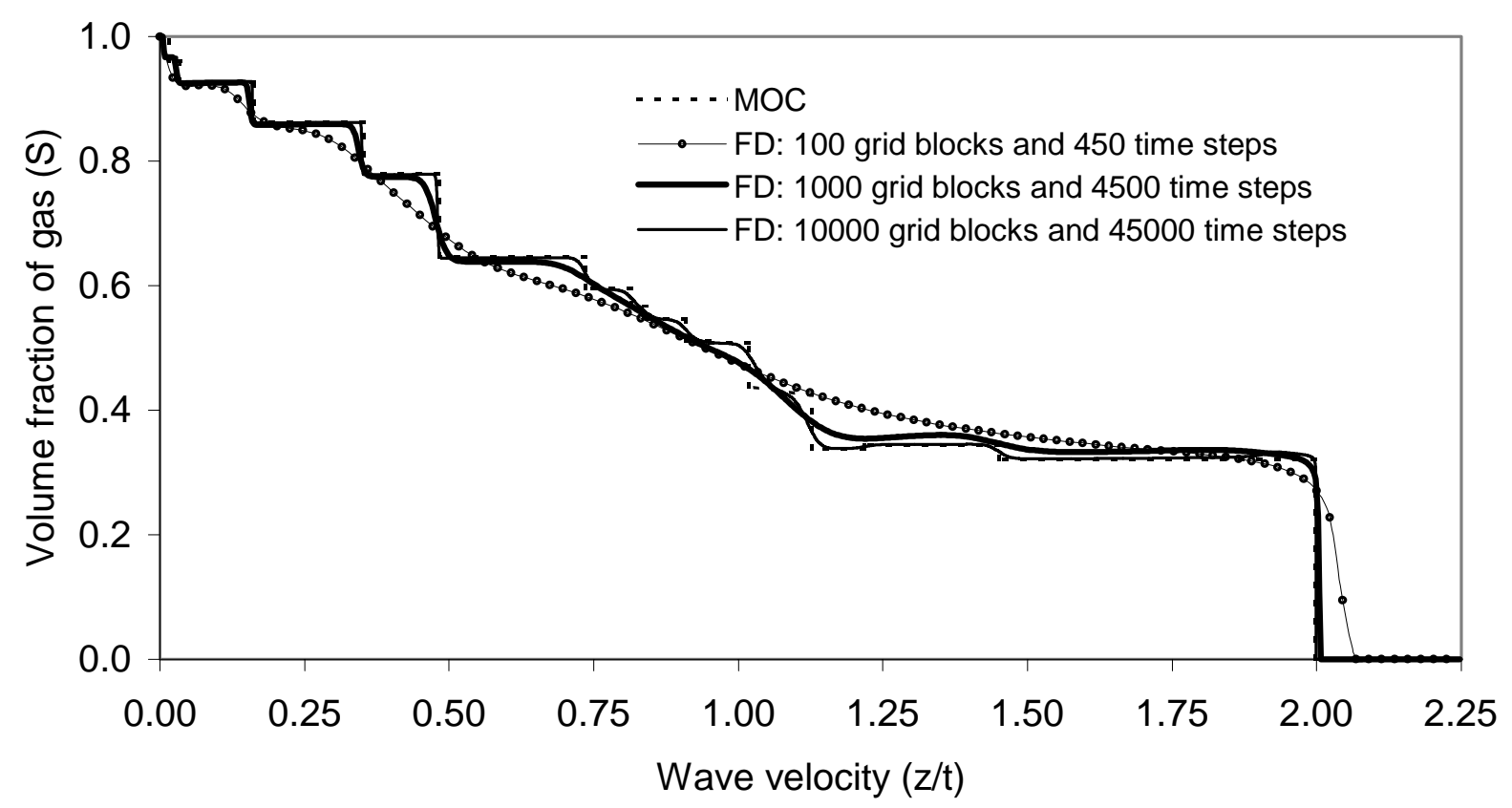

Figure 7.3: Comparison of analytical and numerical saturation profiles for displacement of Oil A by Gas A at $275 \mathrm{~atm}$ and $387.45 \mathrm{~K}$. The finite difference (FD) solutions were obtained with 100, 1000, and 10,000 grid blocks and $\Delta z / \Delta t=10$.

cost for capturing the true dispersion-free saturation profile by numerical simulators is substantially higher.

\subsection{Systems with Nontie-Line Rarefactions}

The shock solution described in the previous sections can be found even when there is a rarefaction connecting one or more pairs of tie lines. In many problems, rarefaction segments that appear are short, and wave velocities change little over the length of the rarefaction. In such cases, the shock solution is an excellent approximation of the actual solution. For problems with longer rarefactions, a more accurate approximate solution can be obtained by the procedure described in this section.

The mass conservation equations of the $1 \mathrm{D}$ flow problem can be rearranged into the eigenvalue problem

$$
(A-\lambda I) e=0,
$$

where $A$ is a coefficient matrix, $I$ is the unit matrix, $\lambda$ is the eigenvalue and $e$ the corresponding eigenvector. The elements of the $A$ are given by

$$
A_{j, i}=\frac{\partial F_{j}}{\partial C_{i}}, i, j=1, \ldots, n_{c}-1 .
$$

At any given point in the two-phase region, the $n_{c}-1$ eigenvalues represent characteristic wave velocities of compositions subject to variation in the corresponding eigenvector direction. Tie lines are eigenvectors and the remaining $n_{c}-2$ admissible directions can be integrated to obtain nontieline paths. When a nontie-line rarefaction exists in the $1 \mathrm{D}$ solution, the solution path switches from a key tie line path and travels along a nontie-line path to end up at a neighboring key tie line. The velocity rule dictates that a path switch from a tie line path to a nontie-line path can 
occur only at an equal-eigenvalue point of mixed type. That is a point in compositional space where a tie line eigenvalue coincides with a nontie-line eigenvalue. For a given tie line the number of equal-eigenvalue points of mixed type is $2\left(n_{c}-2\right)$. Half of them can be disregarded immediately, as they are located on the liquid side of the equivelocity curve. The equal eigenvalue points can be located directly by solving the eigenvalue problem along a given tie line. The direct approach is quite time consuming and hence, the indirect method of Dindoruk (p. 64)[43] for location of equal-eigenvalue points is recommended for problems of the current type.

In the following we assume that two key tie lines, known in advance, are connected by a spreading wave. The question is then: at what equal-eigenvalue point does the tie line path switch to the nontie-line path?

The selection of the appropriate equal eigenvalue point can be done by a geometrical interpretation of the displacement problem. When a rarefaction is present, the key tie lines bound a surface in composition space of tie lines intersected by the path that connects the key tie lines. The nontieline spreading wave traverses that surface. Experience indicates that the tie-line surface is only slightly curved, so it can be approximated well by a plane determined by the key tie lines. Hence, at the correct equal-eigenvalue point only the tie-line eigenvector and the eigenvector related to the matching nontie-line eigenvalue will point in direction of the plane Q spanned by the neighboring key tie lines. In practice this is done by checking angles between the normal vector to $\mathrm{Q}$ and the eigenvectors. Note that for systems with 5 or more components in the mixture, the normal vector to $\mathrm{Q}$ is no longer uniquely determined and must be found by a minimization approach.

After the selection of equal-eigenvalue point, the nontie-line path is traced to the next key tie line by integration of the nontie-line eigenvector. For the general case, however, the presence of a spreading wave is not known in advance. Hence, a tool for predicting the existence of nontie-line rarefactions is needed.

\subsection{Prediction of Spreading Waves in 1D Solutions}

If two key tie lines are connected by a rarefaction, the path switch from the tie line path to the nontie-line path must occur at an equal eigenvalue point on the tie line located closest to the critical locus. This is due to the intrinsic symmetric behavior around critical points. In the work of Dindoruk[43], continuous variation along nontie-line paths is linked to the envelope curve generating the ruled surface traveled by the nontie-line path. This envelope curve is illustrated in a 2-dimensional projection of the general case in Fig. 7.4. The tie lines belonging to the 1-parameter family $\varphi_{k}(\psi)$ on the $k$ th ruled surface are all tangents to the envelope curve $E_{k}$. The overall volume fraction of a given component $i$ at the point of tangency on the envelope curve can be written as

$$
C_{i}=\left(c_{i v}-c_{i l}\right) P_{k}(\psi)+c_{i l},
$$

where $P_{k}(\psi)$ is the superficial vapor volume fraction at the point of tangency. Dindoruk[43] derived an expression for the variation of the nontie-line eigenvalue $\lambda_{k}$ along the nontie-line path in the vicinity of the equal eigenvalue point

$$
\frac{d \lambda_{k}}{d \psi}=\frac{f-S}{\left(S-P_{k}\right)^{2}} \frac{d P_{k}}{d \psi}
$$

where $\lambda_{t}$ is the tie line eigenvalue at the equal eigenvalue point. While Eq. 7.13 applies strictly only near the equal-eigenvalue point, the indicated sign of the left-hand side applies over the entire nontie-line path[43]. 


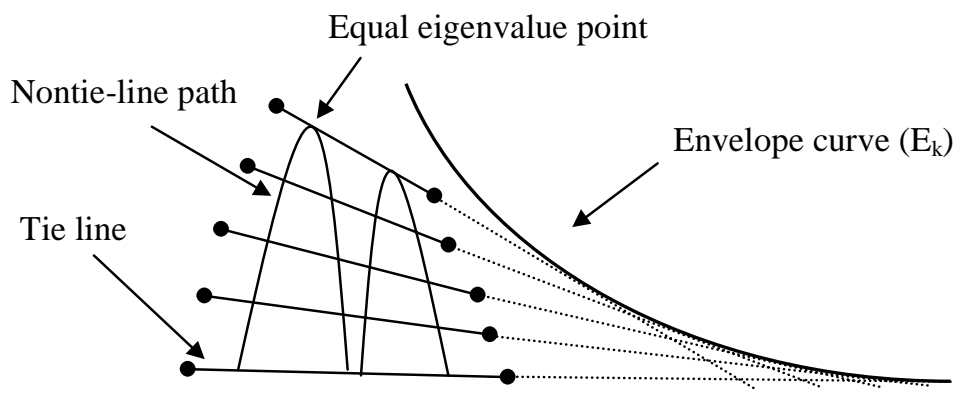

Figure 7.4: Illustration of envelope curve.

\subsection{The Fanning Rule (Envelope Rule)}

Inspection of Eq. 7.12 indicates that a critical point must be a point on the envelope curve. This fact makes it possible to predict the sign of the derivative of the superficial vapor saturation with respect to $\psi$ and hence the variation of $\lambda_{k}$ once the orientation of the key tie lines is known. The absolute distance from the two-phase boundary to the envelope curve increases as the nontie-line path is traced from an equal eigenvalue point. The sign of the superficial vapor fraction depends on whether the tie lines of the ruled surface are fanning from the liquid side or the vapor side of the two-phase region, or equivalently, whether the envelope curve is located in the vapor or liquid side of the two-phase region. Ultimately the shape of the fractional flow curve and the velocity rule are used to determine whether a path switch at an equal eigenvalue point is admissible. Recall that the velocity rule states that a high-speed wave must be found downstream of a low-speed wave. For systems in which the injection composition lies on the vapor side of the phase envelope and the initial composition on the liquid side, the solution path (after the leading shock) lies on the vapor side of the equivelocity curve $(f>S)$. This fixes the sign on the numerator on the right hand side of Eq. 7.13, and hence the derivative of the nontie-line eigenvalue has the same sign as the derivative of the superficial vapor saturation $P_{k}$. Application of the fanning (envelope) rule in combination with the velocity rule result in four distinct cases illustrated in Figs. 7.5-7.8.

The first case illustrated in Fig. 7.5 is a vaporizing wave where the envelope curve is located on the liquid side of the two-phase region. As the nontie-line path is traced from the equal eigenvalue point located downstream $(\mathrm{d})$ towards the injection point located upstream $(\mathrm{u})$, the superficial vapor saturation $P_{k}$ decreases. This is consistent with the velocity rule and hence the path switch is allowed and a spreading wave will be present in the $1 \mathrm{D}$ solution.

Fig. 7.6 shows a condensing wave in which $P_{k}$ is increasing as the nontie-line path is traced from a point upstream $(\mathrm{u})$ towards the initial oil. This is consistent with the velocity rule and hence a spreading wave will form in the solution.

In the condensing drive illustrated in Fig. 7.7, $P_{k}$ is a decreasing function of the nontie-line path when traced from a point upstream $(u)$ towards the initial oil. This is a violation of the velocity rule, as the upstream part of the wave eventually will catch up with the downstream part. In other words, the wave is self-sharpening. The upstream and downstream key tie lines must consequently be connected by a shock. Depending on the compatibility with the solution this can be either a tangent shock or a genuine shock. 


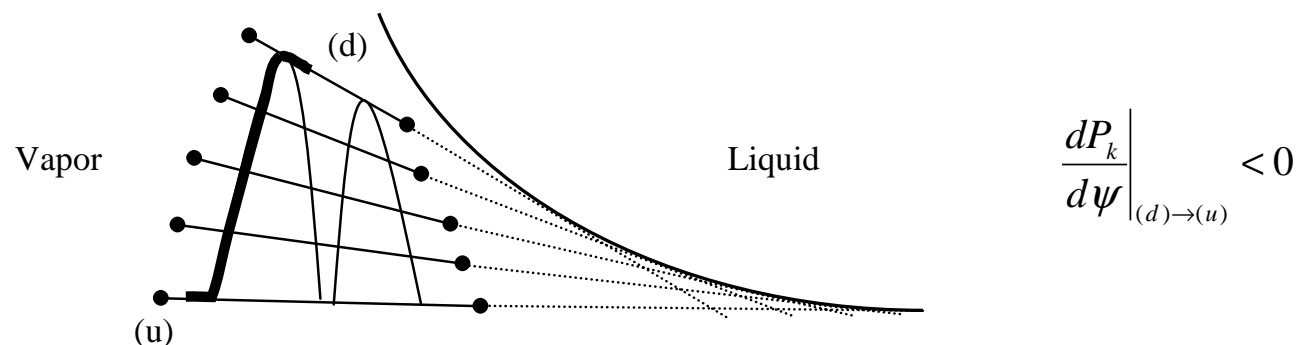

Figure 7.5: Vaporizing wave with liquid side envelope. Upstream (u), Downstream (d).

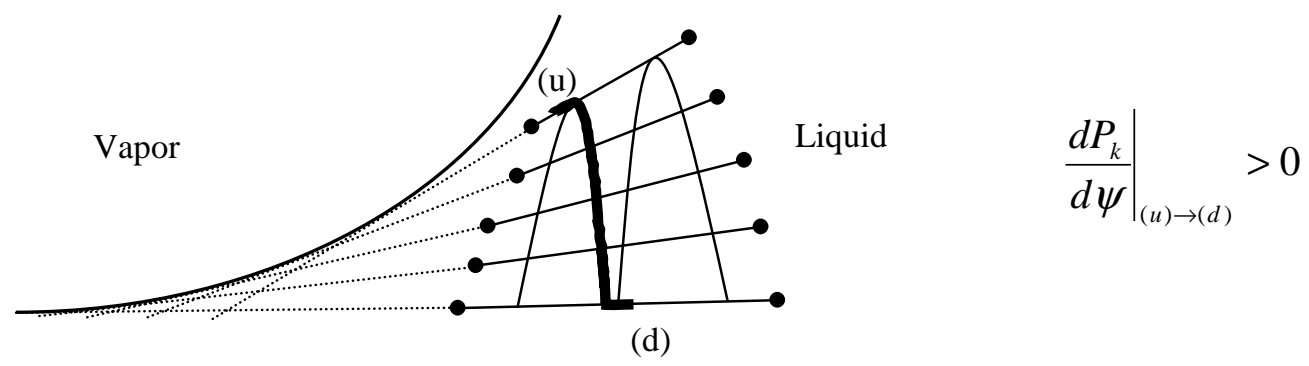

Figure 7.6: Condensing wave with vapor side envelope.

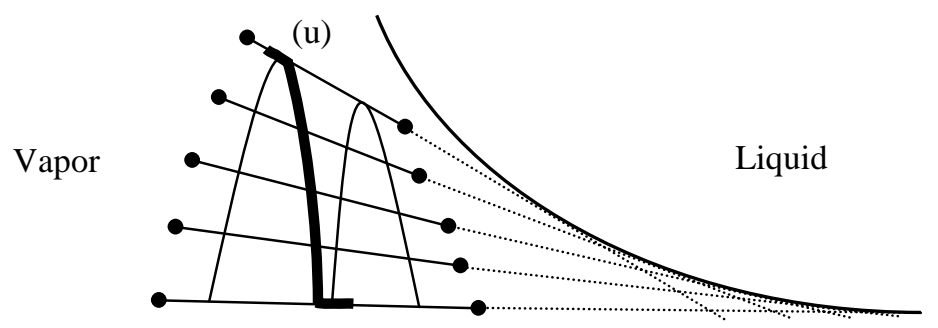

(d)

Figure 7.7: Condensing wave with liquid side envelope. 


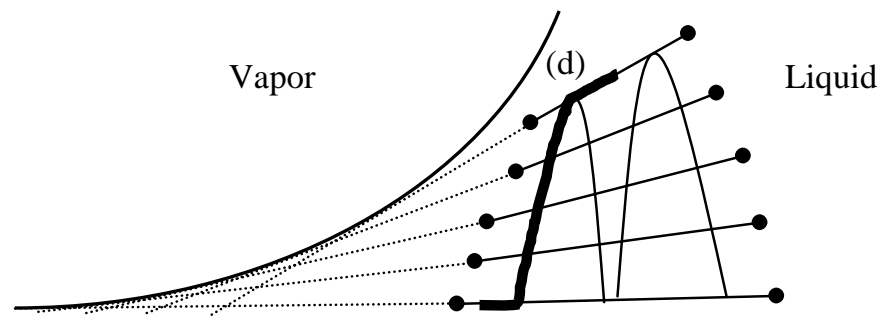

(u)

$$
\left.\frac{d P_{k}}{d \psi}\right|_{(d) \rightarrow(u)}>0
$$

Figure 7.8: Vaporizing wave with vapor side envelope.

\begin{tabular}{||c|c|c|c|c||}
\hline \hline \multirow{2}{*}{ Type } & \multicolumn{2}{|c|}{ Tie line length } & Intersection & \multirow{2}{*}{ Wave } \\
\cline { 2 - 3 } & Upstream & Downstream & & Rarefaction \\
\hline Vaporizing & Long & Short & Liquid side & Shock \\
\hline Vaporizing & Long & Short & Vapor side & Shock \\
\hline Condensing & Short & Long & Liquid side & Rarefaction \\
\hline Condensing & Short & Long & Vapor side & R \\
\hline
\end{tabular}

Table 7.3: Summary of the fanning (envelope) rule.

Fig. 7.8 shows a vaporizing wave with the envelope curve located on the vapor side of the twophase region is inspected. As the nontie-line path is traced from a point upstream $(u)$ towards the injection point, the nontie-line eigenvalue increases. Again this behavior will result in a sharpening wave, a path switch at the equal eigenvalue point is not allowed and a shock is required.

The general feature of the four cases presented above is that a spreading wave only will form if the nontie-line path, starting at the equal eigenvalue point, is moving away from the envelope curve. Whether a rarefaction appears between two key tie lines can be summarized easily in terms of tie line length and whether the intersection point lies on the liquid or vapor side of the two-phase region. Table 7.3 reports that summary. Vaporizing segments occur when a longer key tie line lies upstream of a shorter key tie line (see Figs. 7.5 and 7.8), and in vaporizing segments, a rarefaction occurs when the intersection between the key tie lines lies on the liquid side of the two-phase region. In condensing segments, a shorter key tie line is upstream of a longer one (see Figs. 7.6 and 7.7), and a rarefaction occurs when the intersection is on the vapor side. Additional analysis is required to determine whether rarefactions appear if either the initial oil or the injection gas composition is in the two-phase region.

\subsection{Algorithm for Systems with Nontie-line Rarefactions}

The algorithm for constructing 1D solutions, honoring the existence of spreading waves, is: 
1. Locate all key tie lines by the tie line intersection approach.

2. Apply the fanning rule for each neighboring pair of key tie lines. If no rarefactions are predicted switch to the simplified algorithm for fully self-sharpening systems.

3. For each predicted rarefaction, locate the equal-eigenvalue point and integrate the eigenvalue problem to obtain the corresponding nontie-line path.

4. Locate the primary key tie line and start the shock construction downstream. Switch points between the nontie-line paths and the tie line paths are introduced in the solution requirements in parallel with the velocity rule. The downstream solution is traced until the initial oil composition is reached.

5. Continue constructing the upstream solution by the approach of step 4 until the injection gas composition is reached.

\subsection{Solution Example with Nontie-line Rarefaction}

The appearance of nontie-line rarefactions is commonly observed in the solution path for displacements when $\mathrm{N}_{2}$ is present in the injected gas. To illustrate the limits of the algorithm used for constructing fully self-sharpening solutions, pure $\mathrm{N}_{2}$ is now injected into the reservoir fluid (oil A) at the same temperature and pressure as previously. The saturation profile generated by the algorithm for fully self-sharpening systems is compared with coarse and fine grid numerical simulations in Fig. 7.9. The saturation profile from the fine grid simulation clearly indicates a nontie-line rarefaction between the initial tie line and the first crossover tie line. Fig. 7.9 further illustrates the saturation profile obtained by combining an integration of the nontie-line path with shock constructions as described previously.

For this system, the tie line extending through the initial oil is the primary tie line. Application of the fanning rule indicates that a nontie-line rarefaction connects the initial tie line and the first crossover tie line. The nontie-line path ends at an overall composition approximately located on the adjacent tie line. The inaccuracy of the approximation introduced by assuming intersecting key tie lines is quite small, as Table 7.4 shows. The observed deviation is of an order of magnitude where the numerical evaluation of the coefficient matrix $A$ and the step by step integration may contribute significantly. To avoid any violation of the mass conservation equations, the first crossover tie line was connected to the end point of the nontie-line path by a genuine shock. The downstream solution consists of a continuous variation along the initial tie line and a tangent shock to the initial oil. Genuine shocks and constant states make up the upstream part of the solution until the injection tie line is reached. Finally the solution is completed by a direct jump to the injection composition.

Fig. 7.9 indicates that the fully self-sharpening solution is a much more refined approximation than the one obtained by a coarse grid numerical simulation.

\subsection{Conclusions}

The analysis and examples presented lead to the following conclusions:

1. The tie line intersection approach can be applied to find solutions for 1D-gas displacement problems with an arbitrary number of components in the initial oil and injection gas.

2. The solutions obtained are rigorously correct for fully self-sharpening systems. 


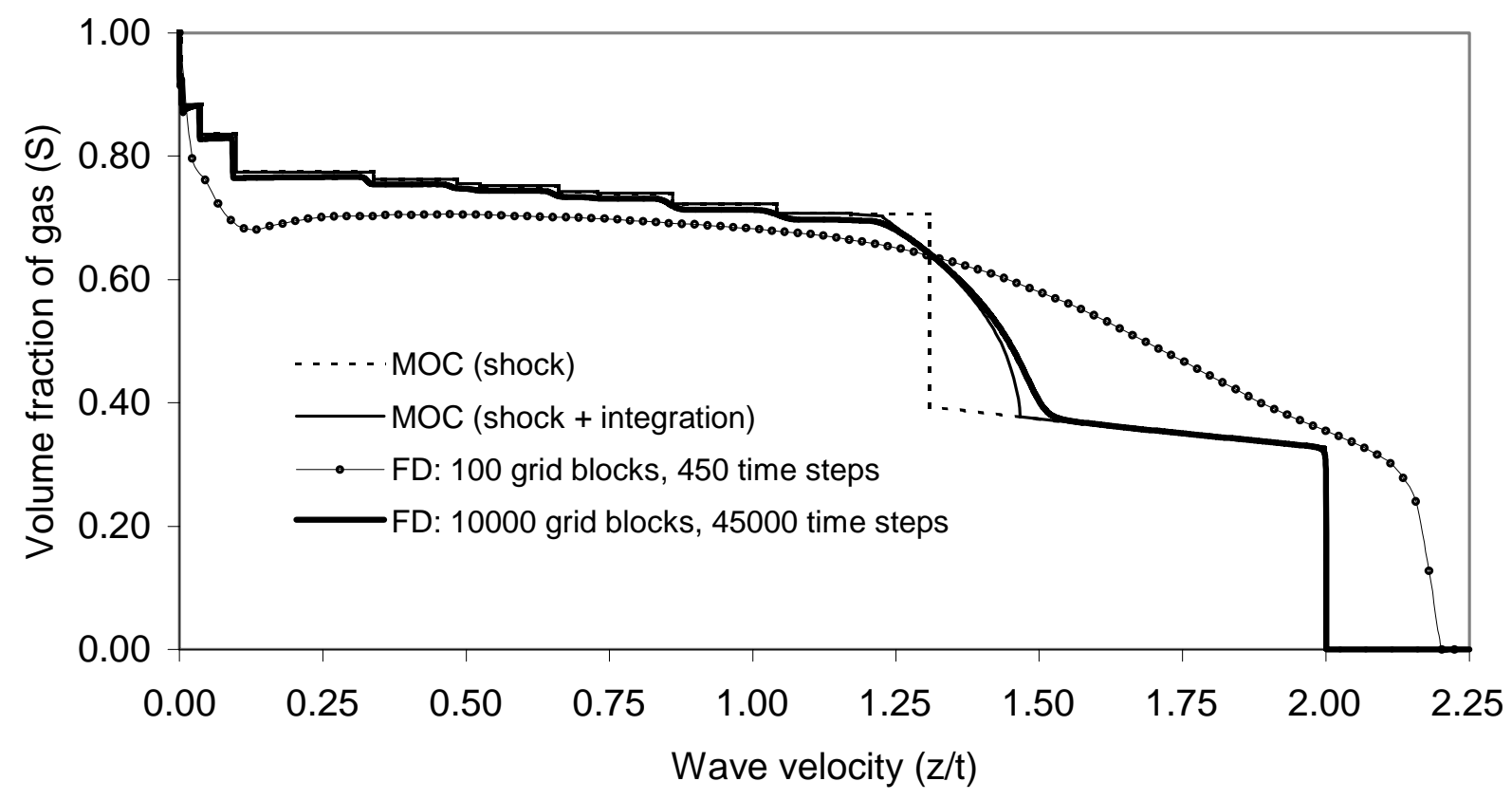

Figure 7.9: Comparison of analytical solutions (with and without integration) with numerical saturation profiles for the displacement of Oil A by pure $\mathrm{n}_{2}$ at $275 \mathrm{~atm}$ and $387.45 \mathrm{~K}$. The finite difference (FD) solutions were obtained using 100 and 10,000 grid blocks and $\Delta z / \Delta t=10$.

\begin{tabular}{|c|c|c|c|c|c|c||}
\hline Comp. & $\mathrm{x}$ (shock) & $\mathrm{x}$ (rarefaction) & Error \% & $\mathrm{y}$ (shock) & $\mathrm{y}$ (rarefaction) & Error \% \\
\hline $\mathrm{N}_{2}$ & 0.23695463 & 0.23657449 & 0.16 & 0.86905145 & 0.87052697 & 0.17 \\
\hline $\mathrm{CO}_{2}$ & 0.00892119 & 0.00896803 & 0.52 & 0.01255008 & 0.01262557 & 0.60 \\
\hline $\mathrm{CH}_{4}$ & 0.00000000 & 0.00000000 & - & 0.00000000 & 0.00000000 & - \\
\hline $\mathrm{C}_{2}$ & 0.06295753 & 0.06196958 & 1.59 & 0.05199391 & 0.05114823 & 1.65 \\
\hline $\mathrm{C}_{3}$ & 0.07434362 & 0.07361960 & 0.98 & 0.03517118 & 0.03476156 & 1.18 \\
\hline $\mathrm{i}-\mathrm{C}_{4}$ & 0.01007617 & 0.00998769 & 0.89 & 0.00333684 & 0.00329854 & 1.16 \\
\hline $\mathrm{n}-\mathrm{C}_{4}$ & 0.03922843 & 0.03896883 & 0.67 & 0.01076864 & 0.01066283 & 0.99 \\
\hline $\mathrm{i}-\mathrm{C}_{5}$ & 0.01382016 & 0.01374001 & 0.58 & 0.00260091 & 0.00257527 & 1.00 \\
\hline $\mathrm{n}_{5} \mathrm{C}_{5}$ & 0.02260102 & 0.02248533 & 0.51 & 0.00376605 & 0.00373032 & 0.96 \\
\hline $\mathrm{C}_{6}$ & 0.03645991 & 0.03630629 & 0.42 & 0.00382992 & 0.00379312 & 0.97 \\
\hline $\mathrm{C}_{7}$ & 0.20213968 & 0.20233382 & 0.10 & 0.00585749 & 0.00581241 & 0.78 \\
\hline $\mathrm{C}_{11}$ & 0.10787320 & 0.10827271 & 0.37 & 0.00095891 & 0.00095155 & 0.77 \\
\hline $\mathrm{C}_{16}$ & 0.08952057 & 0.09020739 & 0.76 & 0.00011148 & 0.00011053 & 0.86 \\
\hline $\mathrm{C}_{23}$ & 0.05901420 & 0.05964260 & 1.05 & 0.00000305 & 0.00000300 & 1.37 \\
\hline $\mathrm{C}_{33}$ & 0.03608969 & 0.03692364 & 2.26 & 0.00000009 & 0.00000009 & 0.88 \\
\hline
\end{tabular}

Table 7.4: Comparison of tie line found by MOC integration and by the tie line intersection approach. Gas A displaced by pure $\mathrm{N}_{2}$ at $275 \mathrm{~atm}$ and $387 \mathrm{~K}$. 
3. The presence of a rarefaction between key tie lines can be determined easily from the lengths of the upstream and downstream tie lines and whether the intersection point lies on the vapor side or the liquid side of the two-phase region.

4. For displacements that include nontie-line rarefactions, the solutions obtained by the tie line intersection approach are approximate, but they are much more accurate than solutions obtained by conventional finite difference compositional simulation unless impractically fine computational grids are used.

5. Times required to obtain approximate analytical solutions by the key tie line method are orders of magnitude lower than corresponding times for conventional compositional simulation.

\subsection{Nomenclature}

$$
\begin{aligned}
A & =\text { Coefficient matrix } \\
C_{i} & =\text { Overall volumetric fraction of component } i \\
c_{i l} & =\text { Volumetric fraction of } i \text { in liquid phase } \\
c_{i v} & =\text { Volumetric fraction of } i \text { in vapor phase } \\
e & =\text { Eigenvector } \\
F_{i} & =\text { Overall fractional flow of component } i \\
f & =\text { Fractional flow of vapor } \\
I & =\text { Identity matrix } \\
M & =\text { Viscosity ratio (vapor/liquid) } \\
n_{c} & =\text { Number of components in mixture } \\
P & =\text { Superficial vapor saturation } \\
S & =\text { Volumetric vapor saturation } \\
\lambda_{t} & =\text { Tie-line eigenvalue } \\
\lambda_{k} & =\text { kth nontie-line eigenvalue } \\
\Lambda & =\text { Shock velocity }
\end{aligned}
$$




\title{
8. Use of Network Modeling to Predict Saturation Paths, Relative Permeabilities and Oil Recovery for Three Phase Flow in Porous Media
}

\author{
Darryl Fenwick and Martin Blunt
}

We present a network model of three phase flow in water-wet porous media. To explain the high oil recoveries in gas injection and gravity drainage experiments, we show that the mechanism for oil recovery is flow through connected oil layers in the pore space that are on the order of a micron thick. We then describe a simple model for the configuration of oil, water and gas in a single pore and present an approximate expression for the conductance of oil layers. We use this expression to derive the oil relative permeability when flow is dominated by layer drainage. We show that for low oil and water saturations $k_{r o} \sim S_{o}^{2}$, consistent with the results of several experiments. To predict $k_{r o}$ for the full range of oil saturation we use a capillary equilibrium based network model that can simulate any sequence of oil, water and gas injection. We introduce a self-consistency procedure to ensure that the correct sequence of saturation changes is used in the network model to compute relative permeability. We then present relative permeabilities and oil recoveries for gas injection into different initial oil saturations, and for waterflooding a reservoir containing gas and oil. We show that the relative permeabilities are strongly affected by the fluid properties and by the type of displacement process.

\subsection{Introduction}

Network models simulate multiphase flow through an idealized representation of the pore space to calculate average properties, such as relative permeability, capillary pressure and oil recovery. Network models can predict multiphase flow properties directly if both the geometry of the porous medium and the displacement process are known precisely [23, $7,151,161]$. Where a complete description of the flow physics and the pore structure is unknown or difficult to obtain, conceptual models can be developed. These models make approximations about the structure of the pore space and the flow processes. While they cannot make direct predictions of multiphase properties, they can be used to provide insight into flow in porous media. With suitable tuning of parameters, the models can match experimental data and can then be used to make predictions for situations outside the range of available measurements. Examples of this approach include studies of relative permeability hysteresis and the effects of wettability in two phase flow [16, 104, 135, 46, 142]. In this report we use a conceptual model to study three phase flow.

The flow of three phases - oil, water and gas - occurs in a variety of different displacement processes in oil reservoirs and during pollutant transport and clean-up. Although there is now a large body of literature on three phase relative permeability (for example, Oak [155] provides a review of studies up to 1990 and Jerauld [103] provides a recent discussion of measurements in Prudhoe Bay), three phase flow is not well understood, and current empirical models for relative permeability do not adequately describe the full range of possible behavior.

A three phase network model requires knowledge of the pore-scale displacement mechanisms, which are studied using micromodels that reproduce the anticipated behavior in real rock. As a result of these experiments the displacement processes for three phase flow in water-wet media are

\footnotetext{
${ }^{7}$ The material in this chapter was published as "Network Modeling of Three-Phase Flow in Porous Media," SPE Journal (1998) 3, 86-97.
} 
now fairly well understood $[30,196,158,156,160,115]$. Based on these observations several three phase network models have been constructed [195, 162, 178, 84, 131, 67, 69]. These models have predicted successfully oil recovery in micromodel experiments [195, 162, 178], and have computed three phase relative permeabilities and capillary pressures $[67,69]$.

In this report we will present a network model for water-wet media and use it to address two unique aspects of three phase flow. First, oil may form a layer in grooves, crevices, roughness or corners of the pore space, sandwiched between water close to the solid surfaces and gas in the center of the pores. Flow through such layers is the mechanism by which oil may drain to low saturation during gas injection. We will present an approximate analytical model of oil layer conductance and use it to predict oil relative permeability in the layer drainage regime. Second, a three phase displacement involves changes in two independent saturations. This is in contrast to two phase flow (say, oil and water), where the water saturation can only increase (imbibition for a water-wet system) or decrease (drainage). The direction of the saturation change affects relative permeability and capillary pressure. In three phase flow there is an infinite number of possible routes in saturation space all with potentially different relative permeabilities and oil recoveries. In our model we specify a sequence of saturation changes. However, in a macroscopic displacement, normally the boundary conditions are known - the gas and water fractional flows at injection wells and the initial saturation of oil and water in the reservoir. This displacement results in a certain sequence of saturation changes at a fixed point in the reservoir, but the network model does not automatically know what the sequence is. In this report we present a self-consistency procedure that allows the network model to find relative permeabilities for the right saturation path.

First we will discuss the significance of oil layers in three phase flow and describe how the network model computes oil layer conductance. Before describing the network model itself, we show how we can make predictions of oil relative permeability directly from expressions for the oil layer conductance. Then we will introduce the network model. Further details are provided elsewhere $[67,66]$. We then describe the self-consistency procedure. Last we will present selfconsistent saturation paths and relative permeabilities for gas injection and for waterflooding a reservoir containing oil and gas.

\subsection{Oil Films and Layers}

Dumoré and Schols [49] showed that low oil saturations can be achieved during gas injection in water-wet rock. Since then, many other authors [114, 217, 113, 194, 227] have studied three phase gas injection and gravity drainage and have all confirmed that very high oil recoveries are possible. Remaining oil saturations as low as $0.1 \%$ have been recorded [227]. The mechanism for these high recoveries is oil drainage through connected layers in the pore space. The presence of oil layers is controlled by the spreading coefficient. The initial spreading coefficient for oil $C_{s}^{i}$ is:

$$
C_{s}^{i}=\gamma_{g w}-\gamma_{o w}-\gamma_{g o}
$$

where $\gamma$ is the interfacial tension of pure fluids before they are brought in contact with each other, and the subscripts $g, o$ and $w$ label gas, oil and water respectively. If $C_{s}^{i}>0$, oil will spread over a flat water surface. If $C_{s}^{i}<0$ it will not. However, fluids in porous media are usually at thermodynamic equilibrium, thus the arrangement of fluid in the pore space is governed by the equilibrium spreading coefficient:

$$
C_{s}^{e q}=\gamma_{g w}^{e}-\gamma_{o w}^{e}-\gamma_{g o}^{e}
$$

where the interfacial tensions are measured for fluids in contact with each other. If $C_{s}^{i}>0$, the gas/water interface will be covered by a film of oil, making $\gamma_{g w}^{e}$ significantly lower than $\gamma_{g w}$. $C_{s}^{e q}$ is 
either zero or negative [1]. $C_{s}^{e q}$, the interfacial tensions and the wettability of the rock control the configuration of fluids in the pore space.

Historically, the experiments analyzing hydrocarbon spreading between water and gas have been performed on flat surfaces. If the hydrocarbon spreads, the resulting film over the water surface has been found experimentally and theoretically to be typically of order molecular size. [99, 39, 66] However, in porous media there are grooves, roughness, and corners. Oil in the pore space does not flow through molecular-sized films, that have a negligible conductance, but instead through layers of order microns thick in the pore space wedged between water and gas, as illustrated in Fig. 8.1(a). In this paper we use the word film only to refer to intermolecular films in contrast to layers that are microns across. Several micromodel experiments have confirmed that the pore scale mechanism of oil recovery is through the drainage of oil layers [30, 196, 158, 156, 115, 159, 47]. Oil layers may be present even for $C_{s}^{i}<0[115,47]$.

We will now make an approximate calculation of how thick the oil layer must be to explain observed drainage rates. Take, as an example, the experiments of Zhou and Blunt [227]. Here oil saturations as low as $0.1 \%$ were achieved after three weeks of hexane drainage in a sand column that had a porosity of approximately 0.35 . The column had length of $1 \mathrm{~m}$. Clearly the drainage rate is highest at the beginning of the experiment and decreases to almost zero by the end. However, to obtain a typical channel size, we assume that the oil saturation in the top half $(0.5 \mathrm{~m})$ of the column decreases by $5 \%$ in one week, which is consistent with the experimental data [227]. The oil flow is the saturation decrease times the porosity times the length of column, or $0.05 \times 0.35 \times 0.5=8.75 \times 10^{-3}$ $\mathrm{m}^{3}$ per $\mathrm{m}^{2}$ per week, which is a Darcy velocity $q=1.4 \times 10^{-9} \mathrm{~ms}^{-1}$. The mean grain diameter of the sand was approximately $d=500 \mu \mathrm{m}$. We assume that the distance between pores is also $500 \mu \mathrm{m}$. Thus the total oil flow in each pore $Q=q \times d^{2}=3.6 \times 10^{-15} \mathrm{~m}^{3} \mathrm{~s}^{-1}$. If we assume that the flow is accommodated by a layer of thickness $t$ and width $d$ (the pore size) flowing freely under gravity, then [124]

$$
Q=\frac{d t^{3}}{3 \mu} \rho_{o} g
$$

where $\mu$ is the oil viscosity (approximately $3 \times 10^{-4} \mathrm{kgm}^{-1} \mathrm{~s}^{-1}$ for hexane) and $\rho_{o}$ is the oil density $\left(659 \mathrm{kgm}^{-3}\right)$ and $g=9.81 \mathrm{~ms}^{-2}$. Solving for $t$ using the data above gives $t \approx 1 \mu \mathrm{m}$. Again this is consistent with our assertion that oil forms a layer of order a micron across in the pore space. It is possible to perform similar calculations for other experiments - the layer thickness is found to be approximately one micron or larger. Since such low oil saturations are reached, these layers must be well connected throughout the porous medium.

The arrangement of the three fluids in a representative corner of the pore space is shown schematically in Fig. 8.1. We assume that the oil layers are connected throughout the porous medium. We also assume that the medium is strongly water-wet, such that the oil/water contact angle $\theta_{\text {ow }}$ is less than or equal to the gas/oil contact angle $\theta_{g o}$ and that the only curvature of the interface is in the plane of the wedge. For this configuration to be possible, $\theta_{o w}+\beta<\pi / 2$ and $\theta_{g o}+\beta<\pi / 2$, where $\beta$ is the half-angle of the corner. The oil/water capillary pressure is $P_{\text {cow }}=\gamma_{\text {ow }}^{e} / r_{\text {ow }}$ where $r_{o w}$ is the radius of curvature of the oil/water interface. Similarly $P_{c g o}=\gamma_{g o}^{e} / r_{g o}$ where $r_{g o}$ is the radius of curvature of the gas/oil interface. A thick oil layer is present when $P_{\text {cow }}$ is large and $P_{\text {cgo }}$ is small. The oil layer is assumed to exist until the contact of the oil/water interface with the solid surface coincides with the gas/oil contact, as shown in Fig. 8.1(b). We define a ratio of interfacial curvatures as:

$$
R=\frac{r_{o w}}{r_{g o}}=\frac{P_{c g o} \gamma_{o w}^{e}}{P_{c o w} \gamma_{g o}^{e}}
$$

If $R$ is very small, the oil layer can be very thick. The critical ratio $R_{c}$ at which the layer is no 


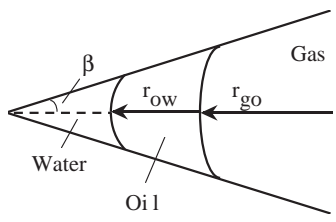

(a)

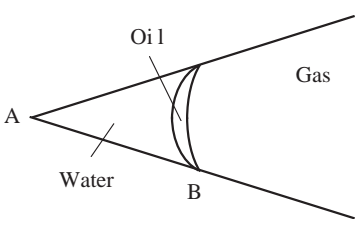

(b)

Figure 8.1: (a) Three phases in a corner with half angle $\beta$. (b) When the gas/oil and oil/water interfaces touch at point $\mathrm{B}$, the oil layer is assumed to be unstable.

longer stable is:

$$
R_{c}=\frac{\cos \left(\theta_{g o}+\beta\right)}{\cos \left(\theta_{o w}+\beta\right)}
$$

$R_{c}$ depends only on $\beta$ and the contact angles. It is independent of the magnitude of the interfacial tensions. If $R<R_{c}$, then the fluid arrangement resembles Fig. 8.1(a). If $R>R_{c}$, there is no oil layer, and there is only a gas/water interface with an interfacial tension $\gamma_{g w}^{e}$. Although there might be a molecular-sized oil film present, this film is too thin to have any significant effect on the flow of oil. [19]

For a completely water-wet system it is possible to relate $\theta_{g o}$ to $C_{s}^{e q}$. It is assumed throughout this work that water coats the entire solid surface and the system is strongly water-wet $\left(\theta_{\text {ow }}=\right.$ $\left.\theta_{g w}=0\right)$. At the gas/oil/water contact, the horizontal force balance of the interfacial tensions is given by,

$$
\gamma_{g w}^{e}=\gamma_{g o}^{e} \cos \theta_{g o}+\gamma_{o w}^{e} .
$$

Eq. 8.6 can be manipulated, resulting in,

$$
\cos \theta_{g o}=1+\frac{C_{s}^{e q}}{\gamma_{g o}^{e}}
$$

as derived by Kalaydjian [112] and Kalaydjian et al. [113]. Using Eq. 8.7, Eq. 8.5 becomes,

$$
R_{c}=1+\frac{C_{s}^{e q}}{\gamma_{g o}^{e}}-\left(-\frac{C_{s}^{e q}}{\gamma_{g o}^{e}}\right)^{1 / 2}\left(2+\frac{C_{s}^{e q}}{\gamma_{g o}^{e}}\right)^{1 / 2} \tan \beta
$$

Eq. 8.8 is based upon a simple geometric argument for oil layer stability. However, the analysis gives an indication of the expected behavior of oil layers. Layers can be present even for negative spreading coefficients, as shown experimentally [47, 115], but as the spreading coefficient decreases, oil layers become increasingly less likely to exist. Note that whether or not a oil layer exists depends on the value of $R$ which is governed by the ratio of capillary pressures, and on $R_{c}$ which depends on $C_{s}^{e q}$. Thus oil layers may be present for $C_{s}^{e q}<0$, and absent for $C_{s}^{e q}=0$, depending on the capillary pressures. 


\subsection{Conductance of Oil Layers}

In this section we investigate the expression for the conductance of an oil layer between water and gas. We define a conductance $g$ where the volumetric flow in a single pore is given by $Q=g \Delta \Phi$, where $\Delta \Phi$ is the potential drop across the pore. The conductance of water in a corner (Fig. 8.1) is given by [183]

$$
g_{w}=\frac{A_{w} r_{o w}^{2}}{\mu_{w} l R_{f w}}
$$

where $A_{w}$ is the area of water in the corner, $l$ is the length of the pore, and $\mu_{w}$ is the viscosity of water. $R_{f w}$ is a constant dimensionless resistance factor for flow of a single fluid in a corner [183]. This value was calculated by an approximate expression by Zhou et al. [229] $A_{w}$ is given by

$$
A_{w}=r_{o w}^{2}\left[\sin \alpha_{w}\left(\cos \alpha_{w}+\sin \alpha_{w} \cot \beta\right)-\alpha_{w}\right],
$$

where

$$
\alpha_{w}=\frac{\pi}{2}-\theta_{o w}-\beta
$$

If we assume that all three phases in the pore are flowing with the same imposed potential gradient and the water and oil viscosities are assumed equal $\left(\mu_{o}=\mu_{w}=\mu\right)$, then the conductance of an oil layer in a corner is the conductance of both water and oil in the corner minus the conductance of the water, or

$$
g_{o}=\frac{A_{c} r_{g o}^{2}}{\mu l R_{f o}}-g_{w}
$$

where $A_{c}$ is the area of water and oil in the corner, $g_{w}$ is given by Eq. 8.9, and $R_{f o}$ is another dimensionless resistance factor whose value is dependent upon the gas/oil contact angle $\left(\theta_{g o}\right)$ on the water surface. We have ignored viscous coupling between the fluids. For a triangular pore with $\beta=30^{\circ}, R_{f w}=31$. If $\theta_{g o}=0$, corresponding to $C_{s}^{e q}=0$ from Eq. 8.7, then $R_{f o}=31$. If $\theta_{g o}=33^{\circ}$, corresponding to $C_{s}^{e q}=-4 \mathrm{mN} / \mathrm{m}$ from Eq. 8.7, then $R_{f o}=70$.

$A_{c}$ is given by

$$
A_{c}=r_{g o}^{2}\left[\sin \alpha_{o}\left(\cos \alpha_{o}+\sin \alpha_{o} \cot \beta\right)-\alpha_{o}\right]
$$

where

$$
\alpha_{o}=\frac{\pi}{2}-\theta_{g o}-\beta
$$

If there is a no flow boundary at the oil/water interface, a different expression for oil layer conductance is appropriate. Approximate analytical expressions for this case are given by Zhou et al. $[229]$

\subsection{Relative Permeability of a Single Pore}

Now that we have an expression for oil layer conductance, we can predict the oil relative permeability. We will assume that the porous medium is a bundle of parallel pores of constant crosssection, where every pore has been entered by gas and oil flow occurs only through layers. This layer drainage regime is thus appropriate for low oil and water saturations. While we cannot use this approach to predict exactly the oil relative permeability for a real rock, we will show that the functional form of the relative permeability is consistent with several experimental measurements.

The saturation of a phase is the fraction of the cross-sectional area of a pore occupied by that phase. Eq. 8.13 gives us the area of water and oil in a corner, $A_{c}$, and hence:

$$
S_{w}+S_{o}=\frac{n_{c} A_{c}}{A_{t}}
$$


where $n_{c}$ is the number of corners in the pore and $A_{t}$ is the cross-sectional area of the pore. Similarly, the water saturation $S_{w}=n_{c} A_{w} / A_{t}$.

If the pore is completely full of one phase, the conductance is approximately [23]:

$$
g_{t}=\frac{\pi r_{e f f}^{4}}{8 \mu l},
$$

where the effective radius $r_{\text {eff }}=1 / 2\left(\sqrt{A_{t} / \pi}+r_{i}\right)$ and $r_{i}$ is the inscribed radius of the pore. The oil relative permeability is $k_{r o}=n_{c} g_{o} / g_{t}$.

Using Eqs. 8.12-8.14 it is possible to find $k_{r o}$ as a function of $S_{w}$ and $S_{o}$. We will spare the details, and give an expression for one example, for a pore of equilateral triangular cross-section $\left(\beta=30^{\circ}\right)$ with $\theta_{g o}=\theta_{o w}=0$. In this case, $n_{c}=3, R_{f w}=R_{f o}=31$, and

$$
k_{\text {ro }}=0.632\left(S_{o}^{2}+2 S_{o} S_{w}\right) \text {. }
$$

If there is no flow boundary condition between oil and water it is possible to show that the oil relative permeability is [229]

$$
k_{r o}=0.632 \frac{S_{o}^{3}}{S_{o}+S_{w}} .
$$

When the oil saturation is much smaller than the water saturation, then the functional form of the oil relative permeability depends on the boundary condition at the oil/water interface: $k_{r o} \sim S_{o}$ if oil and water flow together, Eq. 8.17, while $k_{r o} \sim S_{o}^{3}$ for no flow at the oil/water interface, Eq. 8.18. $k_{r o} \sim S_{o}^{3}$ is consistent with a classic film drainage expression for oil flow, Eq. (3).

For both Eqs. 8.17 and 8.18, when the oil saturation is of the same magnitude or larger than the water saturation:

$$
k_{r o} \sim S_{o}^{2} .
$$

The quadratic form for $k_{r o}$ is readily explained $-S_{o}$ is proportional to the oil area in the corner $\left(S_{o} \sim A_{o}=A_{c}-A_{w}\right)$, while the conductance and hence $k_{r o}$ is proportional to the square of the oil area $\left(A_{o}^{2}\right)$.

The conceptual picture of oil flowing in layers in the pore space leads to the prediction of quadratic oil relative permeabilities at low saturation - a prediction that is confirmed by a number of experiments. Fig. 8.2 shows the oil relative permeability at low saturation from the experiments of Grader \& O'Meara [76] which were performed in glass bead packs with analogue fluids. In these experiments $S_{w} \approx 6 \%$ for $S_{o} \leq 30 \%$, and thus we expect Eq. 8.19 to be valid. At low oil saturation, a best fit of the data gives $k_{r_{o}} \sim S_{o}^{1.9}$, consistent with layer drainage. In other work, gas injection $k_{r o}$ in a consolidated sandstone also showed quadratic behavior [147]. Similar experiments by Goodyear and Jones [75] again showed the same functional form for the oil relative permeability.

This simple analysis cannot, however, predict $k_{r o}$ for larger values of $S_{o}$, where the arrangement of oil-filled pores is important, nor can it determine the effect of spreading coefficient on $k_{r o}$. Network modeling is necessary to predict the effect of spreading coefficient and to compute relative permeabilities for the full saturation range.

\subsection{Network Model}

The network model is a cubic lattice of pores connected by throats. All the pores and throats have equilateral triangular cross-sections. The pore and throat radii are chosen from different distributions. The model consists of $30 \times 15 \times 15$ pores. Fluids are injected at the inlet and displaced 


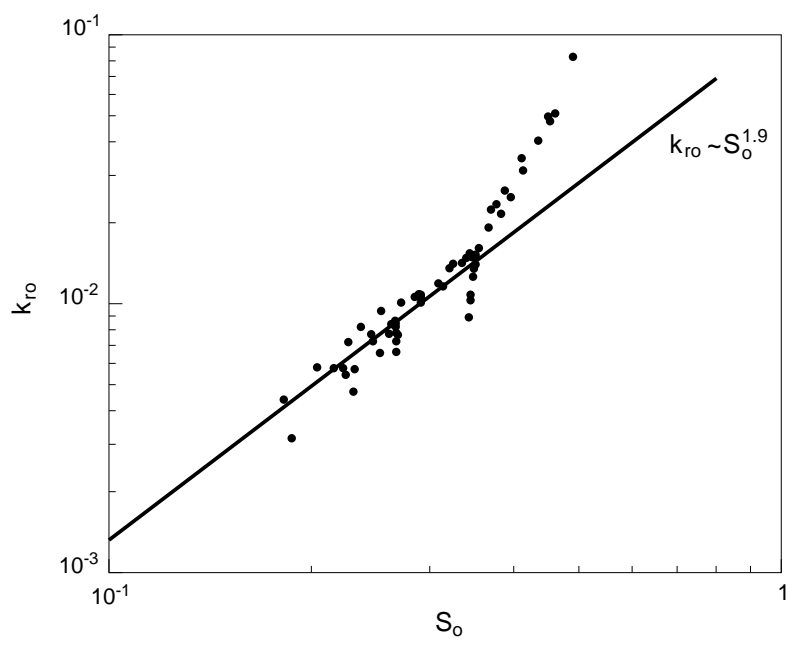

Figure 8.2: Oil relative permeabilities measured in a water-wet system. (from Grader \& O'Meara [76] using a glass bead pack and analogue fluids. Included is a line of slope 1.9 on a $\log$-log scale that best fits the $k_{r o}$ data at low $S_{o}$.)

through the outlet. There are periodic boundary conditions on the other sides. Saturations and relative permeabilities are computed on the final $15 \times 15 \times 15$ section of the model to avoid inlet effects. We did not observe any outlet effects. Several of the results were also calculated for statistically equivalent, but different realizations, and on $40 \times 20 \times 20$ networks. In all cases, the results were similar. However, there are some examples where finite size effects are important, and these will be discussed later. The model is strongly water-wet and $\theta_{o w}=0$. The model allows the two phase displacement processes - piston-like throat filling, cooperative pore filling and snap-off - observed in micromodels by Lenormand et al. and others and incorporated into previous two phase network models (see, for instance $[104,16]$.) For three phase flow the displacement processes observed in micromodels $[158,30,196,115]$, including the presence of oil layers, are added in the model. Further details are given elsewhere $[67,66]$.

We assume that locally the system is in capillary equilibrium, which is appropriate for capillary numbers of around $10^{-7}$ and lower, where the viscous pressure drop across the network model, including the pressure drops in oil layers, are small in comparison with the capillary pressure. A dynamic three phase network model that has explored the effects of pressure gradients in oil layers has been presented by Pereira et al. [178]. Our model is accurate only in the limit of very low flow rates. At every stage in the displacement either water, oil or gas is injected to fill one pore or throat. We compute the capillary pressures for all possible two and three phase displacement mechanisms. From this we can compute the entry pressure for each pore and throat. The entry pressure is the pressure of the injected phase necessary to fill a pore or throat, assuming that the other two phase pressures are held constant. We fill the pore or throat with the lowest entry pressure. In this way we can inject oil, gas and water into the network in any order.

Periodically, we compute relative permeabilities. We find the conductance of each phase in every pore and throat and solve for the phase pressures. There are four possible arrangements of fluid in a pore or throat:

1. A pore or throat full of water. Here use Eq. 8.16 for the conductance.

2. A pore or throat containing gas in the center and water in the corners. For the gas phase use Eq. 8.16 but where $A_{t}$ is replaced by the cross-sectional area of gas in the pore. For water 


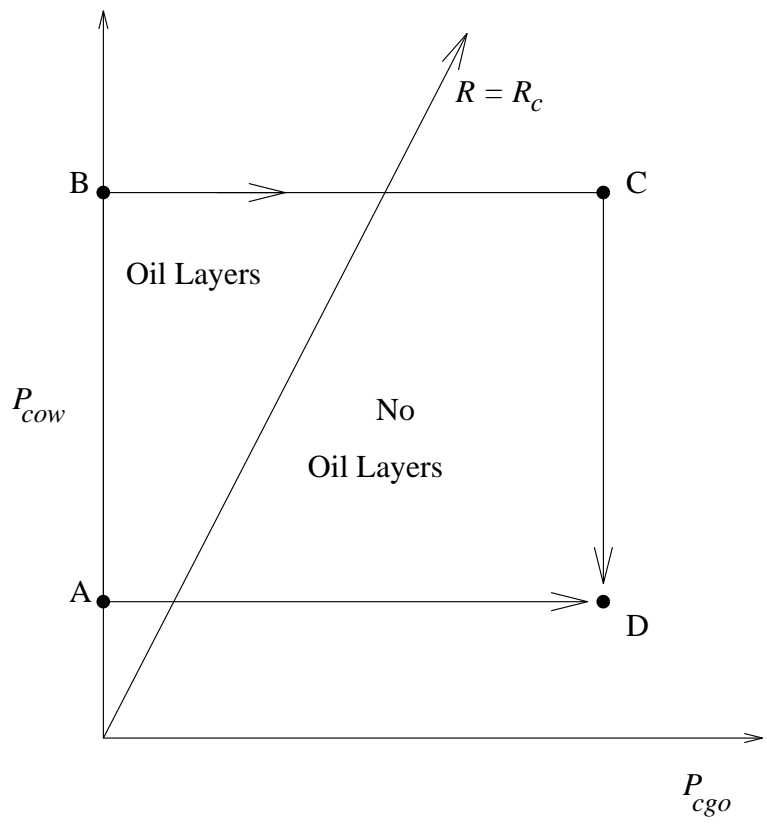

Figure 8.3: Different paths in capillary pressure space. The $R=R_{c}$ line separates the regions of oil layer stability. Path BCD represents gas invasion into high $P_{\text {cow }}$ corresponding to high $S_{o}$, followed by imbibition of water. Path AD represents gas invasion into low $P_{\text {cow }}$ corresponding to residual oil saturation after water invasion.

Eq. 8.9 is used, where $r_{o w}$ is replaced by $r_{g w}$, the gas/water radius of curvature.

3. A pore or throat containing oil in the center and water in the corners. The water conductance is again given by Eq. 8.9, and the oil conductance by Eq. 8.16 but where $A_{t}$ is replaced by the cross-sectional area of oil in the pore.

4. A pore or throat containing gas in the center, water in the corner and an oil layer. An oil layer is only present if $R<R_{c}$, Eq. 8.8. The oil layer conductance is given by Eq. 8.12, the gas conductance is the same as for arrangement (2) and the water conductance is the same as for arrangement (3).

\subsection{Paths in Capillary Pressure Space}

Fig. 8.3 is a schematic diagram representing different three phase displacements as paths in $P_{\text {cgo }}-P_{\text {cow }}$ space. All oil/water displacements occur on the $P_{\text {cow }}$ axis. The point A represents water and waterflood residual oil, while B represents a high oil saturation. When gas is introduced into the system, $P_{\text {cgo }}$ is defined. The line $R=R_{c}$ separates the region where oil layers are stable from the region where oil layers do not exist. $R_{c}$ is calculated from Eq. 8.8 . Let us consider two different paths $\mathrm{ABCD}$ and $\mathrm{AD}$ that start and end at the same capillary pressures.

The path AD represents gas injection into residual oil at a fixed value of $P_{\text {cow }}$. Oil layers are present for only a short range of $P_{c g o}$, and so we expect limited oil layer drainage and little incremental oil recovery. On the other hand, gas injection may result in the formation of an oil bank. At some fixed point in the reservoir, the oil saturation first increases (path AB) before gas enters the system. Path BC represents gas invasion into the high oil saturation in the oil bank, and oil layers exist until a large value of $P_{c g o}$ is reached. In this case there is a greater opportunity for 


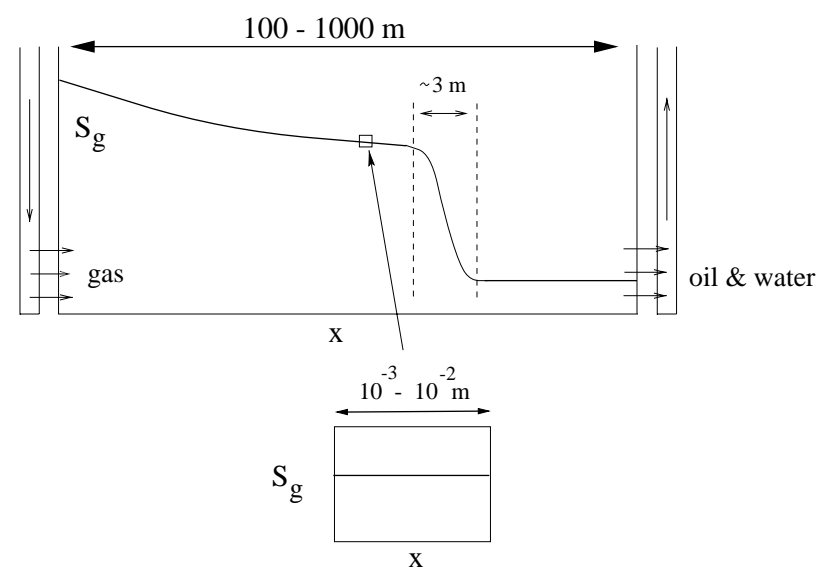

Figure 8.4: Schematic diagram of different scales in multiphase flow in porous media. At the field scale $(100-1000 \mathrm{~m})$, the saturation profile is dominated by viscous forces. At much smaller scales $\left(10^{-3}-10^{-2} \mathrm{~m}\right)$, saturations are relatively constant and flow is dominated by capillary forces. Around a length scale of a meter, capillary and viscous forces are approximately the same.

layer drainage and a lower final oil saturation can be obtained. Path CD represents water injection after gas flooding - while this may give little further oil recovery the oil saturation is likely to be very low already.

In previous work we have used the network model to demonstrate that the route ABCD does indeed give a higher oil recovery than route $\mathrm{AD}[67,69]$. The relative permeabilities and oil recovery are very sensitive to the sequence of saturation changes. This is principally because of oil layers when oil layers are present, oil cannot be trapped, but when they are absent, oil may be trapped by both gas and water, leading to poor oil recovery.

What path does the displacement actually follow? One would hope that a network model would be able to predict the sequence of saturation changes for a given displacement. This issue is discussed next.

\subsection{Self-Consistent Saturation Paths}

Fig. 8.4 illustrates the different length scales for a multiphase flow in a reservoir. The network model represents a small portion of rock over which the saturations are approximately constant and capillary forces dominate. However, over larger scales, the competition between viscous and capillary forces leads to changes in saturation over a few meters. On the reservoir scale, viscous forces dominate. Thus we use a network model that assumes capillary equilibrium to study flow at the pore level. To compute flow on the reservoir scale, we may use a conventional numerical simulator, where to a reasonable approximation capillary forces may be ignored. The link between the pore-level and the field-scale is provided by using relative permeabilities from the network model in the numerical simulator.

The network model computes relative permeability for a particular saturation path. With known initial saturations in the reservoir and injection conditions, we can use the relative permeabilities derived from the network model in a conventional field-scale numerical simulator to predict the sequence of saturation changes in the reservoir. The saturation path computed by the simulator is dependent upon the initial conditions, injection conditions, viscosities of the phases, and gravitational forces. The saturation path from the numerical solver and the saturation path used by the 
network model may not be the same.

If the paths from the network model and the numerical solver are not the same, then the sequence of pore-level displacements does not represent the proper sequence for the particular macroscopic displacement that is being investigated. In order to find the correct saturation path, the iteration procedure shown schematically in Fig. 8.5 is used. The procedure is as follows:

1. With the network model and a specified pore size distribution, perform a three phase displacement with a guessed saturation path from initial to injection conditions.

2. The displacement produces a set of three phase relative permeabilities. The three phase relative permeabilities are tabulated as functions of their own saturation for the selected path.

3. The tabulated values are input into a one-dimensional, three phase Buckley-Leverett numerical solver with given injection conditions and phase viscosities.

4. Stop the simulator after a sufficient amount of time such that any shocks and rarefaction waves are resolved. Find the saturation path by plotting the saturations of all the grid blocks on a ternary diagram. For a Buckley-Leverett problem, the saturation is a function of $x / t$ only. Thus the saturation path measured by taking the saturations in each grid block at a fixed time is the same as the sequence of saturation changes in a fixed grid block over time.

5. Compare the saturation paths of the network model and the numerical simulation. If the saturation values along the two paths differ by less than $1 \%$, we consider them identical.

6. If the paths are not identical, return to step 1 . The saturation path for the network model is that obtained from the numerical solver. If the paths coincide, stop.

When the iteration is complete, the three phase relative permeabilities calculated by the network model are self-consistent. This means that the network model computes relative permeabilities for a sequence of saturation changes that is the same as the sequence that results from a one-dimensional field-scale simulation using the same relative permeabilities.

This procedure gives us valid predictions of relative permeabilities for one-dimensional, homogeneous displacements. However, for heterogeneous, three-dimensional flows, the saturation paths may be different in different regions of the reservoir. Coupling pore scale modeling directly to three-dimensional reservoir simulation remains an unresolved research issue.

\subsection{D Numerical Solution}

The numerical simulations are performed using an explicit, three phase numerical simulator using a total variation diminishing (TVD) scheme. [17] The scheme is second-order accurate in space and first-order accurate in time. Two mass balance equations are solved simultaneously:

$$
\frac{\partial S_{w}}{\partial t}+\frac{\partial f_{w}}{\partial x}=0
$$

and

$$
\frac{\partial S_{g}}{\partial t}+\frac{\partial f_{g}}{\partial x}=0
$$

where,

$$
f_{w}=\frac{\lambda_{w}}{\lambda_{t}}
$$




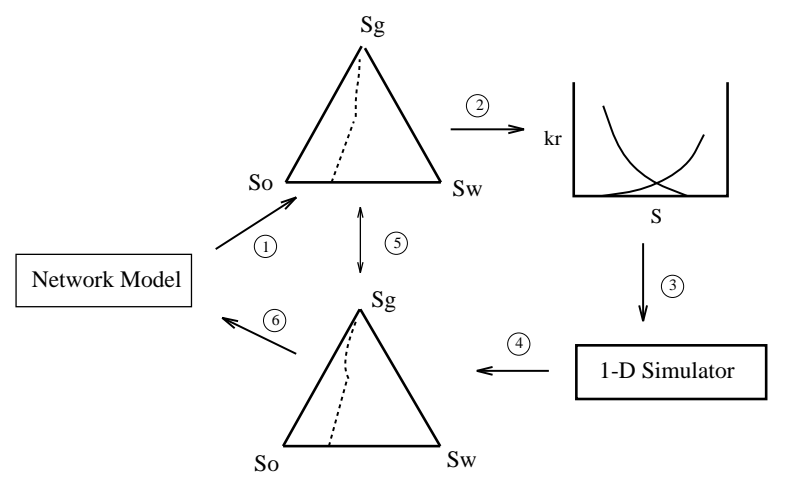

Figure 8.5: Schematic diagram of iteration procedure to obtain self-consistent relative permeability values. (1) A saturation path through the three phase region is guessed and followed with the network model. (2) During the displacement, three phase relative permeability values are calculated. (3) These relative permeability values are input into a 1-D numerical simulator with selected initial conditions, injection conditions, and fluid properties. (4) The 1-D simulator generates another saturation path. (5) This path is compared to the proposed path with the network model. (6) If the paths do not coincide, repeat the procedure, using the path from step 4 in the network model.

$$
\begin{aligned}
f_{g} & =\frac{\lambda_{g}}{\lambda_{t}}, \\
\lambda_{w}=\frac{k_{r w}}{\mu_{w}}, \quad \lambda_{o} & =\frac{k_{r o}}{\mu_{o}}, \quad \lambda_{g}=\frac{k_{r g}}{\mu_{g}},
\end{aligned}
$$

and

$$
\lambda_{t}=\lambda_{w}+\lambda_{o}+\lambda_{g} .
$$

$x$ represents dimensionless distance and $t$ is the number of pore volumes of injected fluid. Eqs. 8.20 and 8.21 assume incompressible, one-dimensional flow where we have ignored gravity and capillary forces. Capillary forces are assumed to be neglible at the field-scale, even though they dominate at the scale of the network model. For the cases we consider we will assume horizontal gas or water injection with no gravity - however, gravity can easily be accommodated in the one-dimensional conservation equations if required.

\subsection{Gas Injection}

We will now present self-consistent relative permeabilities for gas injection into different initial saturations of oil and water. $f_{g}=1$ is specified at the inlet. The viscosities are

$$
\mu_{o}=1 c p, \quad \mu_{w}=1 c p, \quad \text { and } \quad \mu_{g}=0.1 c p .
$$

Note that the self-consistent paths are only valid for this set of phase viscosities.

Runs are performed for $C_{s}^{e q}=0$ and $C_{s}^{e q}=-4 \mathrm{mN} / \mathrm{m}$. $C_{s}^{e q} \approx 0$ represents light alkanes and some oil mixtures, such as Soltrol, while $C_{s}^{e q}=-4 \mathrm{mN} / \mathrm{m}$ is the value for a decane/water/air system [94]. These spreading coefficients span a plausible range for light crude oils at reservoir conditions, although reliable estimates of spreading coefficients in these circumstances are not available.

The displacements start with the network filled with water. Oil is injected until a specified oil saturation is reached. The resulting $S_{w}$ and $S_{o}$ are the initial saturations for gas injection into the 
network, and for the numerical solution. Then gas injection is simulated, and the iteration procedure is followed. Up to 12 iterations may be required until self-consistent relative permeabilities are found, although typically self-consistent paths are found in only four iterations. Note that the self-consistent path may involve the injection of water and oil into the network model, as well as gas.

The relative permeabilities are a function not only of saturation and saturation path, but also depend on the pore scale configuration of fluid. The relative permeabilites here are computed for secondary gas injection - that is gas injection after primary drainage of oil. Tertiary gas injection - gas injection after waterflooding - starts from different pore level arrangements of fluid and thus may give different relative permeabilities.

\section{$\mathrm{C}_{\mathrm{s}}^{\mathrm{eq}}=0$ Results}

Fig. 8.6 shows the self-consistent saturation paths for gas injection into five different combinations of initial oil and water saturations (denoted $S_{o i}$ and $S_{w i}$ respectively). As a comparison Fig. 8.6 also contains experimental saturation paths obtained by Grader \& O'Meara [76]. Grader \& O'Meara used analogue fluids - n-decane $(\mu=0.735 \mathrm{cp})$ was used in place of gas, and benzyl alcohol $(\mu=4.984 \mathrm{cp})$ in place of oil. The water viscosity was $1.140 \mathrm{cp}$. For this fluid system, $C_{s}^{i}=2 \mathrm{mN} / \mathrm{m}$. It is assumed that $C_{s}^{e q} \approx 0$. The porous medium was a glass bead pack, with n-decane (gas) invading into different initial saturations of benzyl alcohol (oil). Fig. 8.2(a) shows $k_{r o}$ for the same experiments.

No attempt has been made to match the experiments. The pore size distribution used in the network model is not intended to be representative of a glass bead pack, and the viscosities of the fluids are different. The experimental data is provided for qualitative comparison only. However, several common features between the network model results and the experiments can be noted:

- At high oil saturations, oil is more mobile than water. Thus, the gas preferentially invades into the oil until $S_{o}$ is significantly reduced.

- Similarly, when $S_{o}$ is low, the gas preferentially displaces the more mobile water until $S_{w}$ is low.

- Different initial conditions follow similar paths in the three phase region, especially at low values of $S_{o}$.

An important difference between the experiment and the network model results is that Grader \& O'Meara observed a shock in the two phase region ahead of the invading gas (n-decane) for an initial oil (benzyl alcohol) saturation of $48 \%$. Two phase shocks are not found with the network model for the gas injection cases studied.

Fig. 8.7 shows the capillary pressure paths. Notice that the paths remain in the region of oil layer stability and thus oil is not trapped, and eventually it can all be displaced. Comparison of Fig. 8.7 with Fig. 8.3 indicates that the capillary pressure paths taken in Fig. 8.3 do not correspond to field-scale gas injection. Paths such as in Fig. 8.3 that force certain values of $P_{\text {cow }}$ or lower to be maintained, and allow oil to be trapped, must correspond to a displacement where both gas and water are injected to displace oil. Only through the injection of water can a fixed value of $P_{\text {cow }}$ be maintained.

Fig. 8.8 shows the self-consistent gas relative permeabilities for the saturation paths shown in Fig. 8.6. The $k_{r g}$ curves are very similar, and virtually identical at higher saturations. Experiments by Oak [154] also found that $k_{r g}$ values were similar at high $S_{g}$ for various initial conditions. Note that at low gas saturations, the curves have a step-like character, which is due to the finite size of the network. 

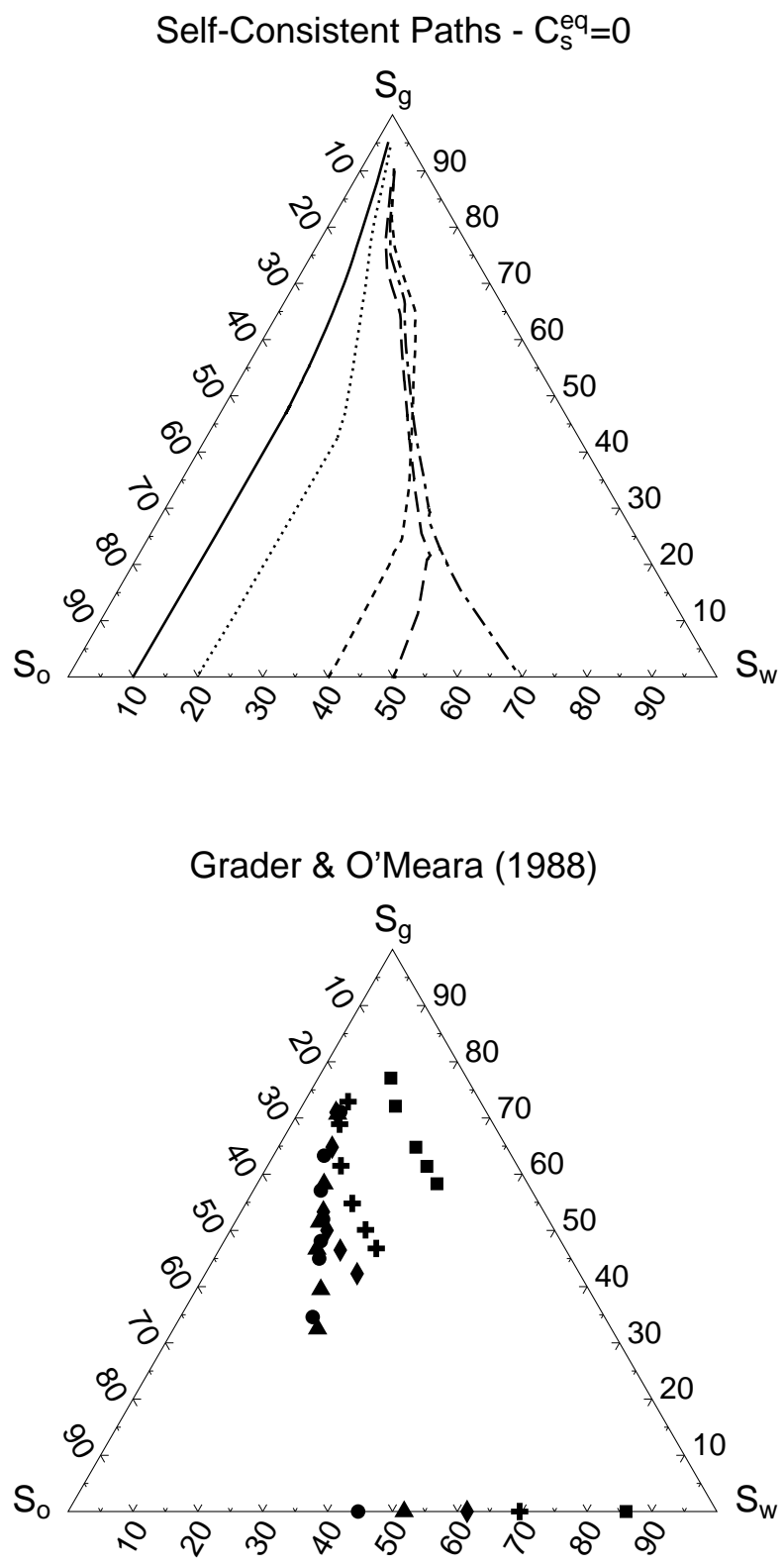

Figure 8.6: Top plot: Self-consistent saturation paths for gas injection into oil and water. Bottom plot: Gas injection experiments performed by Grader \& O’Meara [76] using a glass bead pack and analogue fluids. The experimental data is provided for qualitative comparison only. 


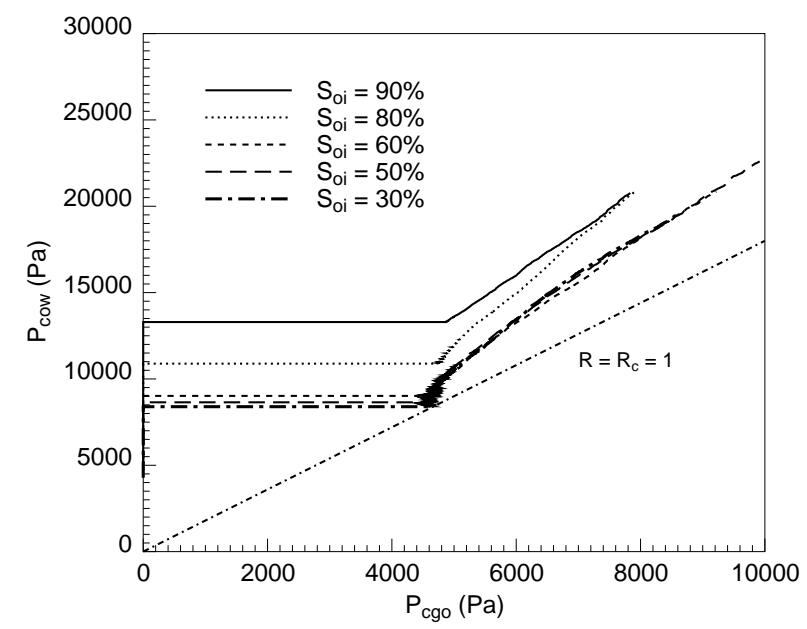

Figure 8.7: Capillary pressure paths for gas injection into different values of initial oil saturation $\left(S_{o i}\right)$.

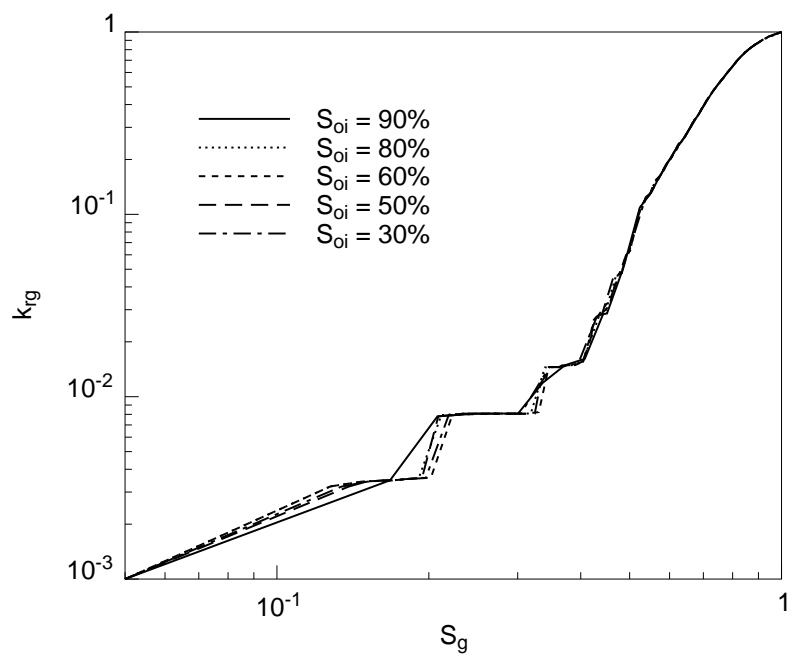

Figure 8.8: Self-consistent $k_{r g}$ curves for the saturation paths in Fig. 8.6. Note that the step-like character of the $k_{r g}$ curves at low $S_{g}$ is due to finite-size effects. 


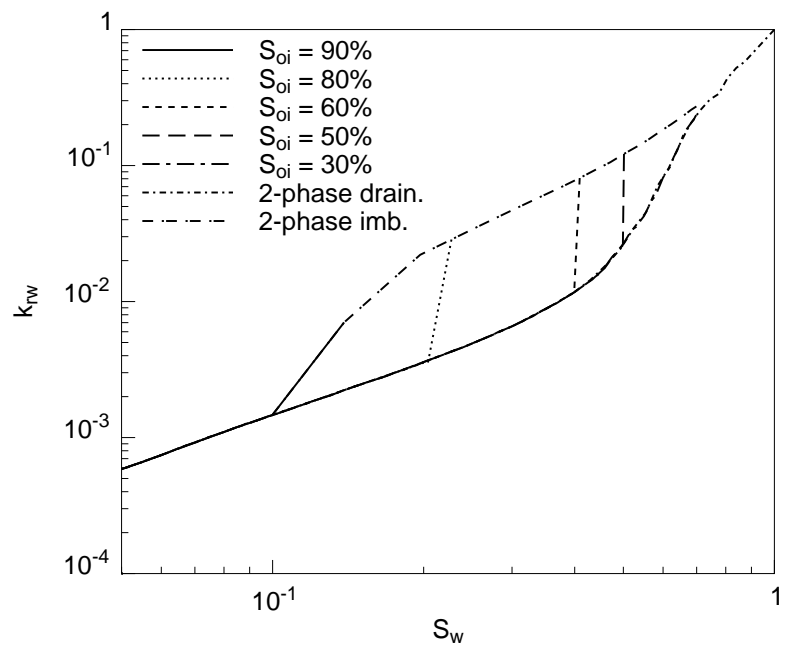

Figure 8.9: Self-consistent $k_{r w}$ curves for the saturation paths in Fig. 8.6. The curves overlap when flow is dominated by water drainage through the corners of the pore space.

The self-consistent water relative permeability curves are given in Fig. 8.9. For reference, the two phase relative permeability curves are also included. If the self-consistency procedure required values of $k_{r w}$ at $S_{w}>S_{w i}$, the two phase imbibition $k_{r w}$ values were used. For $S_{w}<S_{w i}$, the $k_{r w}$ curves are very similar for all values independent of $S_{o i}$, and correspond to the $k_{r w}$ curve for two phase drainage. Several three phase experiments have also shown that $k_{r w}$ curves are similar for different initial conditions. [127, 152, 154, 155] At low $S_{w}$ the drainage curves show a straight line on the $\log -\log$ plot, indicating that the flow is dominated by flow in corners.

Fig. 8.10 shows the self-consistent oil relative permeability curves. There is a very rapid change in $k_{r o}$ at large values of $S_{o}$, which is controlled by the arrangement of pores and throats filled with oil, followed by a less dramatic decrease at low $S_{o}$, in the layer drainage regime. At low $S_{o}$ the $k_{r o}$ curve has a slope of between 1 and 2 on the log-log plot in accordance with the expression for oil layer conductance, Eq. 8.12. The shape of the $k_{r o}$ curve is similar to the experimental results in Fig. 8.2. Unlike the $k_{r w}$ and $k_{r g}$ curves, $k_{r o}$ is different for different initial conditions. This indicates that $k_{r o}$ cannot be written as a function of $S_{o}$ alone. Similar functional forms for $k_{r o}$ have been found experimentally [6, 127, 152, 154, 155].

To illustrate why $k_{r o}$ is a function of both $S_{o}$ and $S_{o i}$, consider $k_{r o}$ at $S_{o}=0.4$ for $S_{o i}=0.9$ and $S_{o i}=0.6$. From Fig. 6 , when $S_{o}=0.4, S_{w}=0.1$ and $S_{g}=0.5$ for $S_{o i}=0.9$, while for $S_{o i}=0.6$ $S_{w}$ is larger (0.4) and $S_{g}$ is smaller (0.2). To a good approximation gas occupies the largest pores and water the smallest, while oil fills those of intermediate size. This means that higher water saturations force oil to occupy larger pores. Hence for $S_{o i}=0.6$ the oil occupies larger pores than for $S_{o i}=0.9$, which in turn leads to a larger $k_{r o}$. Experiments by Skauge et al. [193] have also found that $k_{r o}$ increases for decreasing $S_{o i}$. This effect is evident in Fig. 8.10. However, this example ignores the effect of oil flow through layers, which influences the $k_{r o}$ values in Fig. 8.10. If $S_{o i}$ is low, the oil can be very poorly connected and have a very dentritic structure which tends to reduce $k_{r o}$ disproportionately. This also affects $k_{r o}$. Thus, the $S_{o i}=30 \%$ curve in Fig. 8.10 is below the curves for $S_{o i}=80 \%$ and $S_{o i}=60 \%$.

The finite size of the network affects the $k_{r o}$ calculations. Some of the curves in Fig. 8.10 have been smoothed by interpolation between selected points before input in the numerical simulator, to ensure that the numerical model was stable. 


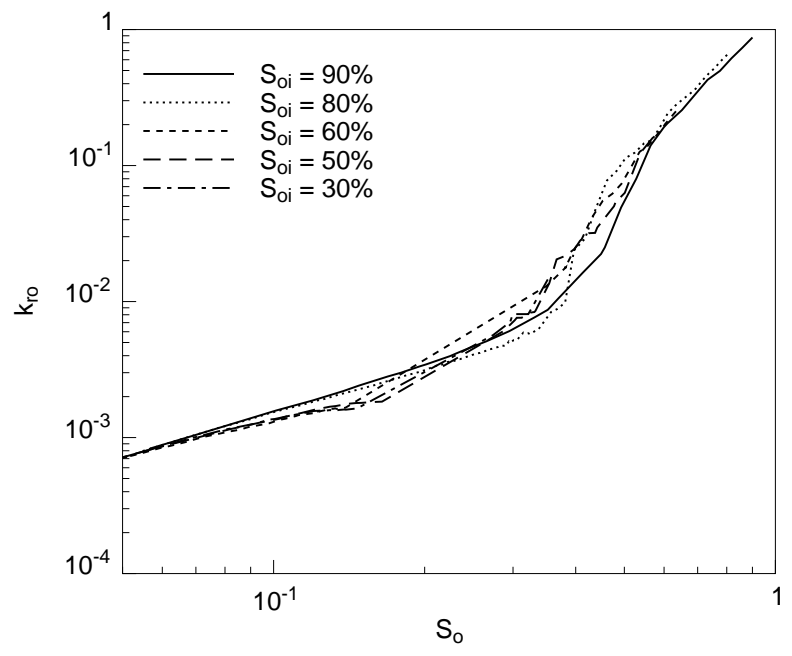

Figure 8.10: Self-consistent $k_{r o}$ curves for the saturation paths in Fig. 8.6. Flow at low oil saturation is governed by flow through layers. When oil flows through oil-filled pores and throats, the relative permeability curves differ for different initial conditions. Note the qualitative similarity with the experimental results in Fig. 8.2.

Fig. 8.11 shows the average oil saturation over all the grid blocks in the numerical solver as a function of the pore volumes of gas injected. The plots are determined by the TVD solution with 500 grid blocks. Eventually all the oil can be recovered, but only after the injection of a huge amount of gas.

\section{$\mathrm{C}_{\mathrm{s}}^{\mathrm{eq}}=-4 \mathrm{mN} / \mathrm{m}$ Results}

Displacements for $C_{s}^{e q}=-4 \mathrm{mN} / \mathrm{m}$ were simulated for initial conditions at $S_{o i}=90 \%$ and $S_{o i}=30 \%$. Fig. 8.12 gives the self-consistent saturation paths. For comparison, the $S_{o i}=90 \%$ and $S_{o i}=30 \%$ paths for $C_{s}^{e q}=0$ are included. The $S_{o i}=30 \%$ path stops at an oil saturation of $23.4 \%$. The $S_{o i}=90 \%$ path is similar to the path at $C_{s}^{e q}=0$. At high $S_{g}$ the $C_{s}^{e q}=0$ path displaces more oil, which is due to the effect of $C_{s}^{e q}$ upon $k_{r o}$ values at low $S_{o}$.

Again there is no trapped oil. For $S_{o i}=30 \%$ when the displacement was stopped, all the oil is surrounded by gas, with water found only in the corners of the pore space. If the displacement in the network model were allowed to continue the capillary pressure path would proceed along the $R=R_{c}$ line, very slowly displacing all the oil from the network.

The self-consistent $k_{\text {ro }}$ values are shown in Fig. 8.13. At high $S_{o}$, the $S_{o i}=90 \%$ curves are very similar for both values of $C_{s}^{e q}$. At low $S_{o}$, the $C_{s}^{e q}=-4 \mathrm{mN} / \mathrm{m}$ curve lies consistently below the $C_{s}^{e q}=0$ curve. Oil flow through layers for $C_{s}^{e q}=-4 \mathrm{mN} / \mathrm{m}$ has a smaller conductance because the ratio of interfacial curvatures $(R)$ is much closer to the critical value $R_{c}$ than for the displacement at $C_{s}^{e q}=0$. The lower $k_{r o}$ values will have an impact upon the rate of oil recovery. For the $S_{o i}=30 \%$ displacement at $C_{s}^{e q}=-4 \mathrm{mN} / \mathrm{m}$, oil layers are not stable all the time. Thus, the oil that reconnects at the beginning of the displacement becomes disconnected again as the displacement continues, similar to the AD path discussed in Fig. 2. The final oil saturation when the displacement ceases is $23.4 \%$. For this condition, a simple linear interpolation from the last finite $k_{r o}$ value to $k_{r o}=0$ at $S_{o}=23.4 \%$ is added. Note that the $k_{r o}$ value at $S_{o}=23.4 \%$ should be finite, reflecting the very slow displacement of oil as the capillary pressure path oscillates along the $R=R_{c}$ line for oil layer stability. Notice that the absence of the layer drainage regime for 


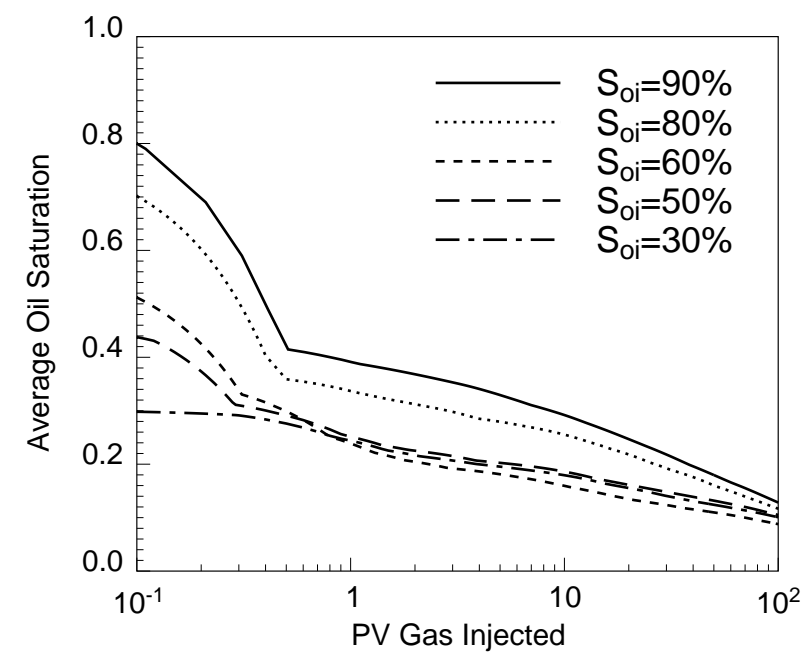

Figure 8.11: The average oil saturation during gas injection for $C_{s}^{e q}=0$. The curves were calculated using the TVD solver with 500 grid blocks.

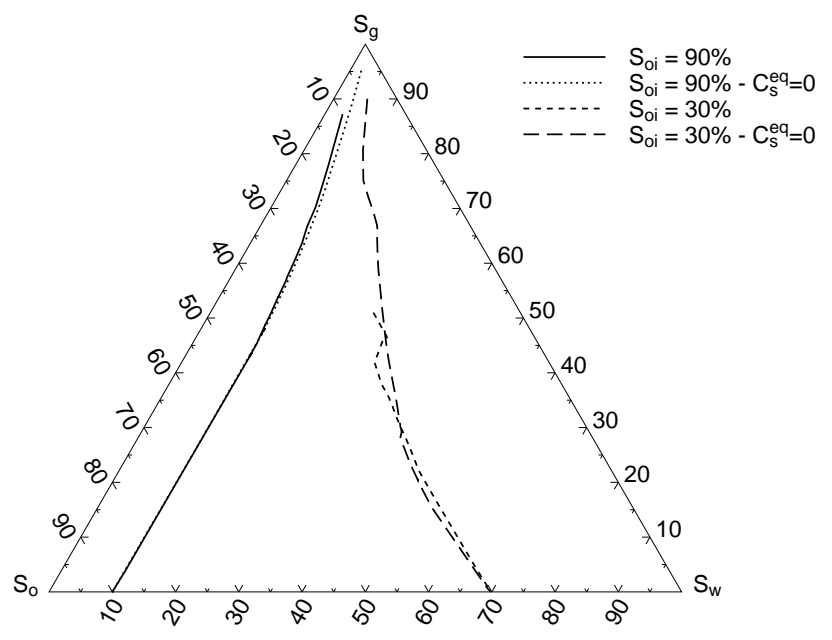

Figure 8.12: Self-consistent saturation paths for gas injection into oil and water with $C_{s}^{e q}=-4$ $m N / m$, with gas injection into $S_{o i}=90 \%$ and $S_{o i}=30 \%$ for $C_{s}^{e q}=0$ included for comparison. 


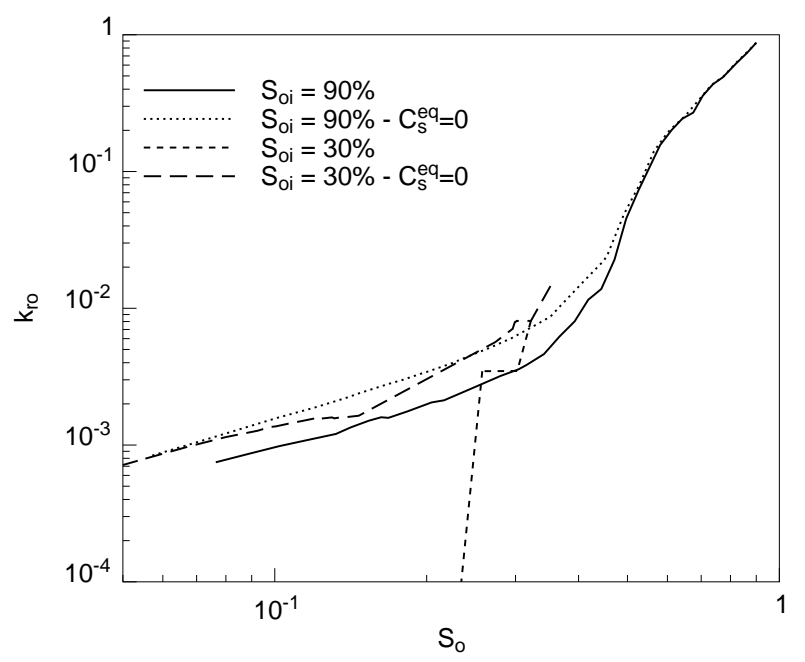

Figure 8.13: Self-consistent $k_{r o}$ curves. At low $S_{o}, k_{r_{o}}$ values for $C_{s}^{e q}=-4 \mathrm{mN} / \mathrm{m}$ are lower because the displacement is closer to the region of layer instability than for $C_{s}^{e q}=0 . k_{r o}$ values for gas injection into $S_{o i}=30 \%$ are not finite throughout the entire displacement. However, oil is displaced to $23.4 \%$ saturation. The $k_{r o}$ curve is constructed by interpolating the last finite value of the $k_{r o}$ curve to zero at $S_{o}=23.4 \%$.

$S_{o i}=30 \%$ is qualitatively in agreement with the experimental measurements shown in Fig. 2(d). In comparison with $C_{s}^{e q}=0, k_{r g}, k_{r w}$, (not shown here) and $k_{r o}$ are all more sensitive to $S_{o i}$. This is due to the more complex behavior when $\theta_{g o}>0$ and oil is not completely wetting in the presence of gas.

Fig. 8.14 shows the recovery curves for the $S_{o i}=90 \%$ and $S_{o i}=30 \%$ paths at the two $C_{s}^{e q}$ values. Recovery is more rapid for $C_{s}^{e q}=0$ due to the higher $k_{r o}$ values at low $S_{o}$.

Figs. 8.11 and 8.14 illustrate an important point regarding the concept of oil recovery and residual oil saturation. For the gas injection examples given, there is no residual oil saturation. However, an immense quantity of gas is required to reduce the oil saturation to very low values. This finding suggests perhaps that reported measurements of $S_{\text {or }}$ for gravity drainage and gas injection experiments are incorrect, and that $S_{o}$ would continually decrease if the experiment were allowed to continue. Fig. 8.14 also illustrates the sensitivity of oil recovery to $C_{s}^{e q}$. Fig. 8.14 indicates that after approximately 10 pore volumes of gas is injected into $S_{o i}=30 \%$, the fraction of original oil in place recovered for $C_{s}^{e q}=0$ is twice the amount for $C_{s}^{e q}=-4 \mathrm{mN} / \mathrm{m}$.

\subsection{Three Phase Waterflood}

Self-consistent three phase relative permeabilities for water injection into a reservoir containing water, oil and gas are presented. $f_{w}=1$ is specified at the inlet, and the viscosities are the same as is used for gas injection. A single run is shown representing the waterflood of a reservoir containing oil and gas. For this case, $C_{s}^{e q}=0$. The displacements start with the network filled with water. Gas invades into the network up to $S_{g}=80 \%$. Oil subsequently invades into the network, displacing the gas, until $S_{o}=30 \%, S_{g}=50 \%$, and $S_{w}=20 \%$. This is the initial condition of the reservoir. Oil is found in layers and in the centers of some intermediate-sized throats and pores. At this point, water injection commences, and the self-consistency procedure is followed. The results given below were determined in three iterations. Fig. 8.15 shows the saturation path. Point $\mathrm{A}$ is the initial 


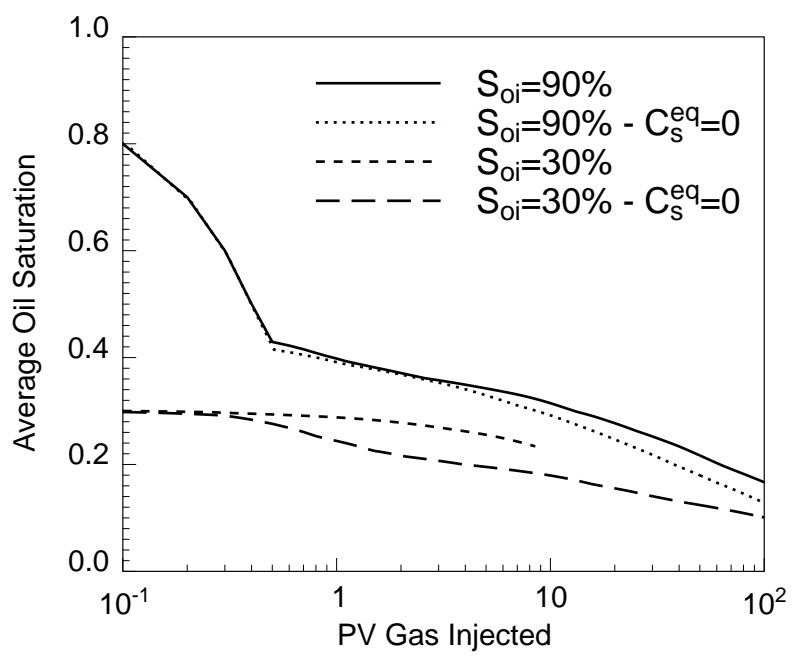

Figure 8.14: The average oil saturation during gas injection for $C_{s}^{e q}=-4 \mathrm{mN} / \mathrm{m}$. The $S_{o i}=90 \%$ and $S_{o i}=30 \%$ paths for $C_{s}^{e q}=0$ are included for reference. The curves were calculated using the TVD solver with 500 grid blocks.

condition. The final saturations are given by point C. At point C, $S_{w}=47.4 \%, S_{o}=7 \%$, and $S_{g}=45.6 \%$ - both oil and gas are trapped. The path from A to Point B represents an oil bank ahead of the invading water front. Note that the advancing oil bank traps the gas, and the oil is subsequently trapped by the advancing water. The capillary pressure path is given in Fig. 8.16. Note that the displacement occurs in the region of oil layer stability. Since oil layers are stable, the oil acts as a wetting fluid. Thus, the oil can rapidly disconnect the gas by snap-off. At B, the gas is trapped. Water invades into oil. At $\mathrm{C}$, oil layers are stable, but some oil is trapped because the gas is also trapped - oil can only flow if there are connected paths of pores that are either oil-filled or contain oil layers.

The oil relative permeability curve for the self-consistent path in Fig. 8.15 along with the $k_{r o}$ for gas injection into $S_{o i}=90 \%$ are shown in Fig. 8.17. The $k_{r o}$ curves are very different. Significant hysterisis is also seen in the gas and water relative permeabilities.

Fig. 8.18 shows the average oil saturation as a function of pore volumes of water injected. Oil recovery is slow initially when the gas is being displaced. As more gas becomes trapped, the oil production increases until the oil is trapped. The ultimate recovery of oil is $76.7 \%$ as a fraction of original oil in place. Note that the rate of recovery is more rapid than gas injection (see Fig. 8.11). Although the ultimate recovery of oil through gas injection is higher, over 100 pore volumes of gas would have to be injected to reach the same average oil saturation.

\subsection{Conclusions}

1. The mechanism for high oil recovery during gas injection is drainage of connected oil layers in the pore space. These layers are of order microns in thickness, wedged between water and gas in the pore space. Such layers can exist even if the spreading coefficient is negative.

2. Layer drainage leads to a characteristic form for the oil relative permeability at low saturation. If both the oil and water saturations are small $k_{r o} \sim S_{o}^{2}$, with no residual oil saturation, as observed in many experiments. 


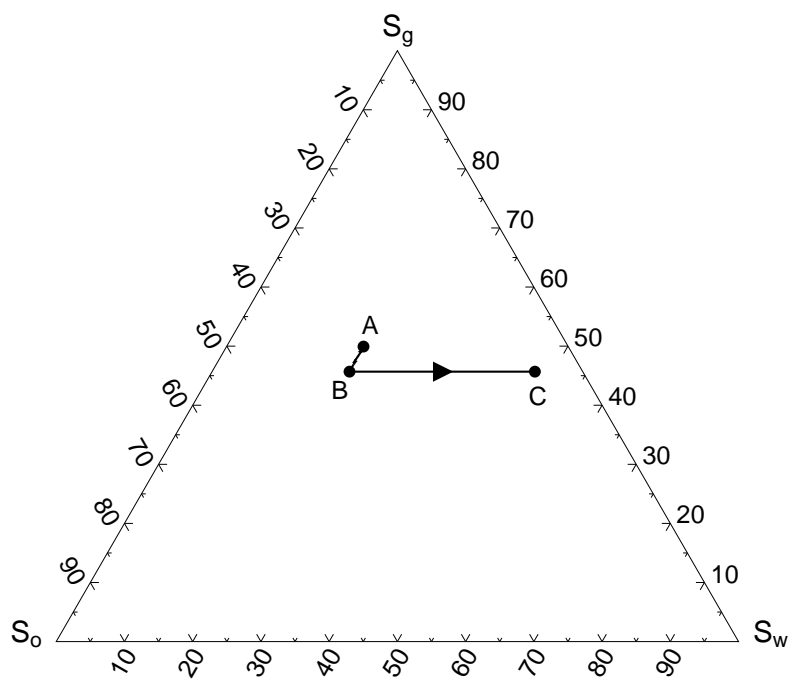

Figure 8.15: Self-consistent saturation path for imbibition into $S_{o}=30 \%, S_{w}=20 \%$, and $S_{g}=50 \%$. Point A is the initial condition. The path from A to B is an oil bank ahead of the water front. Point $\mathrm{C}$ is the final saturations where both oil and gas are trapped. At point C, $S_{w}=47.4 \%, S_{o}=7 \%$, and $S_{g}=45.6 \%$.

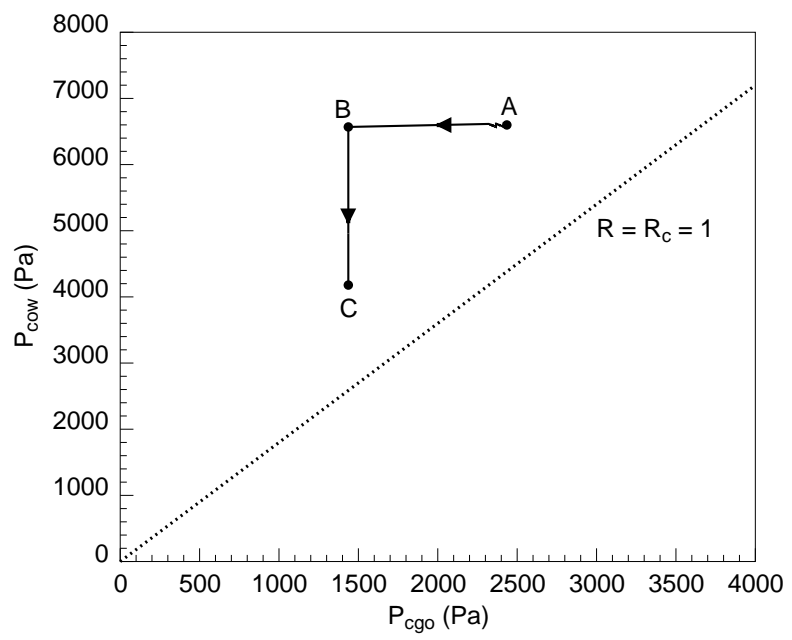

Figure 8.16: Capillary pressure path for the waterflood gas condensate example. Point $\mathrm{A}$ is the initial condition. Point $\mathrm{C}$ is the final condition at which oil and gas are both trapped. The path from $\mathrm{A}$ to $\mathrm{B}$ is an oil bank ahead of the invading water. 


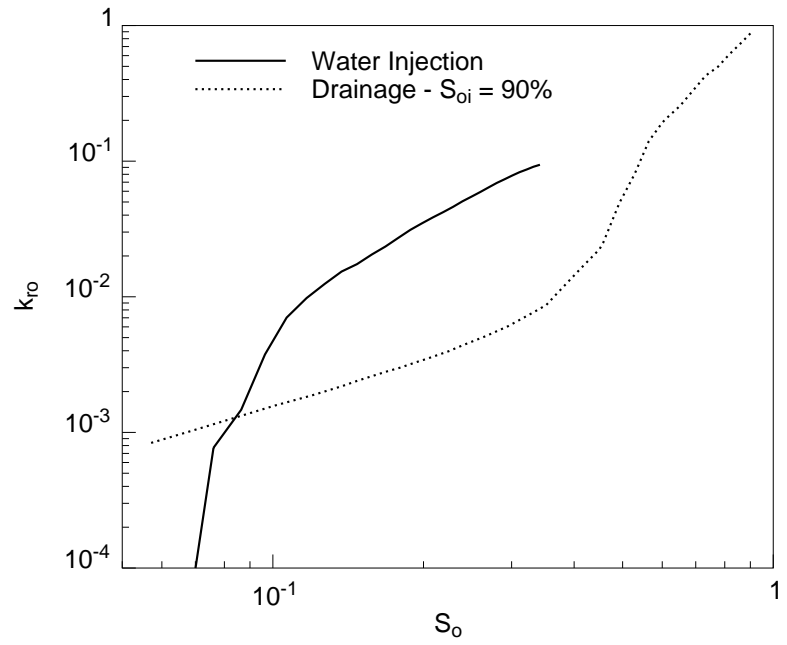

Figure 8.17: The self-consistent $k_{r o}$ curve for water injection into a gas-condensate reservoir. $k_{r o}$ for gas injection into $S_{o i}=90 \%$ is included for comparison.

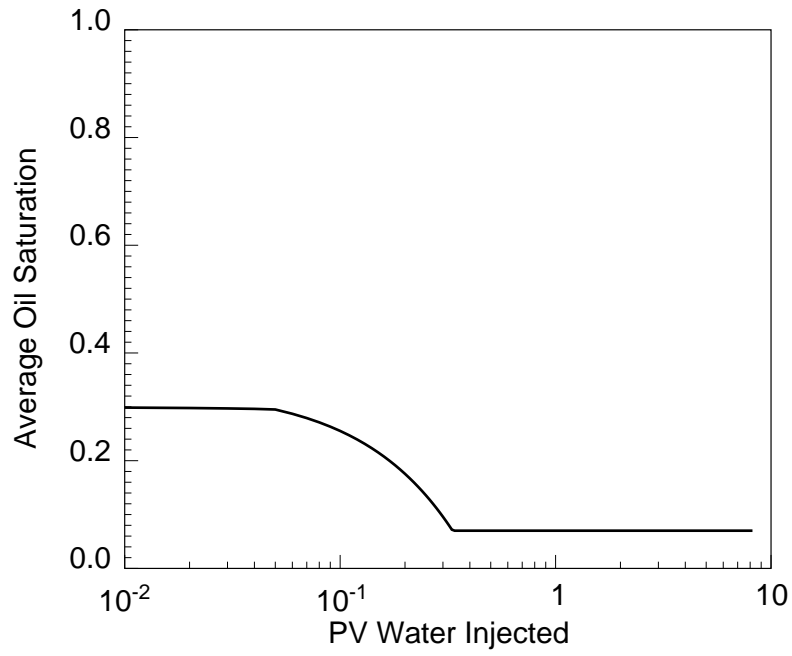

Figure 8.18: The average oil saturation for water injection of a gas condensate reservoir. 
3. The computation of self-consistent saturation paths and relative permeability allows the network model to make predictions of multiphase flow properties for any type of displacement process.

4. The saturation paths and relative permeabilities computed for gas injection into different initial oil saturations show qualitatively similar features to experimental measurements. In particular, for a given fluid system, the water and gas relative permeabilities are similar for different initial conditions, while the oil relative permeability is not. The oil relative permeability shows a rapid decrease with oil saturation at large $S_{o}$ and then a transition to the layer drainage regime.

5. The relative permeabilities and the rate of oil recovery are functions of equlibrium spreading coefficient.

6. The oil, water and gas relative permeabilitites for gas injection are all different from those for three phase waterflooding.

The next few chapters present experimental tests of some of the predictions of the network model, namely the quadratic form of $k_{r o}$ at low $S_{o}$, and the effect of the spreading coefficient. 


\title{
9. Three-Phase Flow Experiments
}

\author{
Akshay Sahni, Dengen Zhou, Jon Burger and Martin Blunt
}

This research presents a framework for analyzing three phase flow experiments in porous media. We analyze data from a previously published dynamic displacement experiment [76], where three phase relative permeabilites have been measured. Once the three phase relative permeabilities are known, we calculate analytically the saturation paths and recoveries for the experiments.

\subsection{Motivation}

The simultaneous flow of three immiscible fluids in porous media is an essential component of enhanced oil recovery and aquifer remediation processes. It is important to understand three phase flow for designing optimum methods for recovering oil by gas injection, gas gravity drainage, surfactant flooding and thermal recovery.

At Stanford, we are trying to get a better understanding of three phase flow, from the pore scale upwards. Over the past few years, research on three phase flow has primarily focussed on:

- two and three phase gravity drainage in capillary tubes

- three phase flow in micromodels

- three phase network modeling

- mathematics of three phase flow

From three phase flow in capillaries we can study the flow of oil as thin layers in an idealized context [229]. Micromodel studies of three phase flow allow us to study pore level processes [115]. Three dimensional and three phase network modeling can give us estimates of relative permeability and capillary pressures for a variety of different three phase flow processes and for different fluid systems [68]. Finally, if we can correctly predict the three phase relative permeabilities, we have the mathematical ability to compute the saturation paths and recoveries analytically $[80,81,79]$.

This research provides a good conceptual picture of several three phase flow phenomena, yet, it is still essential to look at three phase flow behavior in an actual porous medium. Although work has been done to understand three phase flow at the core scale, most of the published literature does an inadequate job of analyzing three phase flow experiments. It is our objective to bridge this gap between experiment and theory of three phase flow in porous media.

At Stanford, we now have a unique experimental capability - a dual energy CT scanner that can operate in both horizontal and vertical mode. This enables us to obtain in-situ measurements of phase saturations and compute three phase relative permeabilities. To study three phase flow at the core scale, we propose to perform and model analytically a series of three phase gravity drainage experiments in both consolidated and unconsolidated porous media. We will also look at systems with different spreading and initial conditions. Then we hope to relate the results obtained from our experiments to those obtained from capillary tubes, micromodels and network modeling. We also extend our theoretical framework to analyze three phase dynamic displacement experiments [76] and construct solutions to three phase flow problems analytically.

\subsection{Literature Review}

Although three phase flow experiments are hard to perform, there are many published studies. Grader and O'Meara [76] performed dynamic displacement experiments using three immiscible fluids. Virnovsky [216] and Grader and O'Meara [76] developed a theory to obtain three phase relative 
permeability as a function of saturation by an extension of the Welge [222] and JBN [111] methods to three phases. Siddiqui et al. [192] verified the theory using X-ray computerized tomography to obtain in-situ saturations for three phase dynamic displacement experiments. Sarem [189] obtained three phase relative permeability by unsteady state displacement experiments assuming that relative permeability of each phase was a function of its own saturation. Oak et al. [155] presented a steady-state study of three phase relative permeability using fired Berea cores. Minssieux and Duquerroix [141] analyzed water alternating gas experiments in porous media with residual oil.

Three phase gravity drainage experiments have shown that it is possible to obtain very low residual oil saturations [49, 114, 113, 227]. Vizika and Lombard [217] studied the effects of wettability and spreading characteristics of the fluid system in three phase gravity drainage. Skurdal et al. [194] analyzed gas gravity drainage experiments using spreading and non-spreading systems under oil wet, water wet and mixed wet conditions. Naylor et al. [147] performed gravity drainage experiments by measuring in-situ oil and brine saturations using a radioactive tracer technique. Chalier et al. [28] used a gamma-ray absorption technique to obtain three phase relative permeability for tertiary gas gravity drainage experiments. Skauge et al. [193] summarized results from gas gravity drainage experiments at different water saturations. Espie et al. [56] established and interpreted laboratory data quantifying the dynamics of oil bank growth during the waterflood/gravity drainage interaction in Prudhoe Bay cores.

In three phase flow, oil may form layers in crevices and roughness of the pore space, between water and gas. It is the drainage of these layers that is responsible for the good recoveries observed in gravity drainage experiments (Blunt et al. [19]). Although the residual oil saturation is very low, the relative permeability at low oil saturations may also be very small, making gas injection schemes uneconomic over any reasonable time scale. It is therefore important to have a good understanding of the three phase relative permeability, especially at low oil saturation.

Most numerical models of three phase flow in porous media use empirical relationships for capillary pressure and relative permeability [64, 168, 200, 201, 211]. Baker[6], Delshad and Pope [40], Oak et al. [155] and Fayers and Matthews [65] compared empirical models to published experimental data and showed that in most cases the empirical models fail to match the measurements.

In the next section 'Dynamic Displacement Experiments', we describe our analysis of the displacement experiment of Grader and O'Meara [76]. We then present our analysis of gravity drainage experiments and detail the proposed research.

\subsection{Dynamic Displacement Experiments}

\section{Overview}

We use data from a dynamic displacement experiment (Grader and O'Meara [76]). The method of characteristics (MOC) is used to calculate analytically saturation paths and recoveries for three phase displacements in one dimension once the relative permeabilities are known (see Guzman and Fayers [80, 81] and references therein for a discussion of MOC theory applied to three phase flow). In contrast with previous work we find MOC solutions for experimentally measured relative permeabilities, rather than assuming, a priori, some empirical model. In other words, rather than attempting to force a pre-conceived model on the data, in this work we find the simplest functional form of relative permeability that adequately matches the data.

\section{Experiment Description}

The fluids used in Grader and O'Meara's experiments [76] were water, benzyl alcohol and decane, and the porous medium was composed of glass beads with 140-200 mesh sizes. Decane (viscosity $=0.735 \mathrm{cp}$ ) was used in place of gas, to reduce viscous fingering effects, and benzyl alcohol was the 


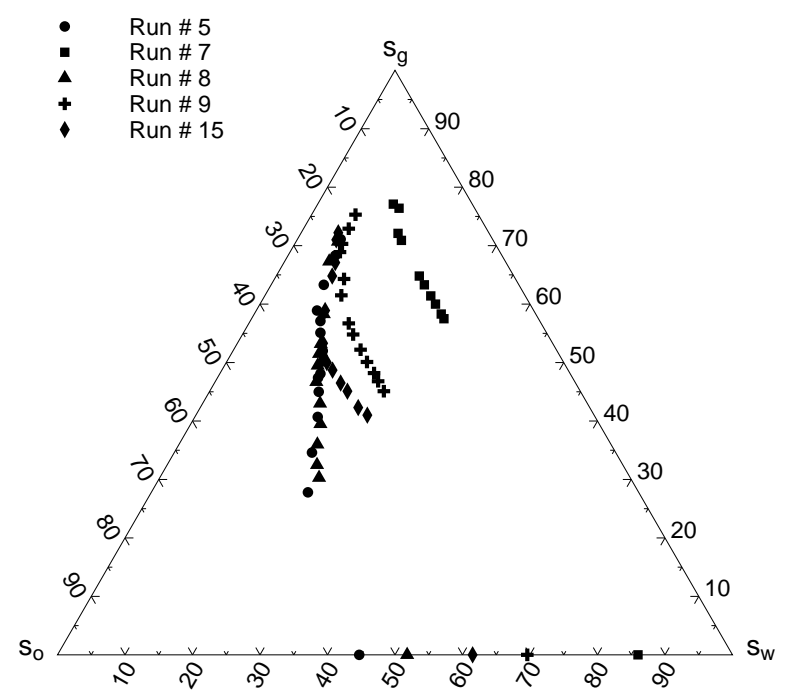

Figure 9.1: Saturation trajectories from Grader and O'Meara's [76] experiments

oil phase. The viscosities of water and benzyl alcohol were $1.140 \mathrm{cp}$ and $4.984 \mathrm{cp}$ respectively. The initial spreading coefficient for oil was $0.2 \mathrm{mN} / \mathrm{m}$. The interfacial tensions were about an order of magnitude less than typical gas-oil-water systems to reduce capillary end-effects. A three phase dynamic displacement experiment was initiated by saturating the porous medium totally with water and then flooding it with oil until the irreducible water saturation was reached. Uniform two phase saturation in the core was obtained by injecting a given fractional flow of oil and water to reach steady state. At this point, the injection of gas (decane) was started.

Virnovsky [216] and Grader and O'Meara [76] extended the Welge/JBN [222, 111] method for determination of two phase relative permeability to three phases. Details of calculating the saturation of a particular phase at the outlet end and the corresponding phase relative permeability are provided in these papers. Several experiments were performed with different initial water saturations, and the saturation trajectories obtained are shown on the ternary diagram, Fig. 9.1.

\section{Analysis of the Experiment}

The three phase relative permeabilities obtained by Grader and O'Meara are shown plotted as a function of their own saturations in Fig. 9.2. To a good approximation the relative permeability values lie on a single curve and hence appear to be a function of their own saturation.

In order to have a convenient functional form of relative permeabilities, we matched them to the following polynomial:

$$
k_{r p}=A s_{p}+B s_{p}^{2}+C s_{p}^{3}+D s_{p}^{4}+E s_{p}^{5}
$$

where the subscript $p$ labels the phase (oil, water or gas).

The matching parameters $A, B, C, D$ and $E$ were found by a least-squares fit to the data. Table 9.1 shows the values obtained. If Eq. 9.1 predicts $k_{r p}<0$, we set $k_{r p}=0$. Recently Hicks et al. [89] analyzed similar experiments and showed that Stone model I and II [200, 201] relative permeabilities could not match the experimental recoveries very well. They got better results by using different Corey [36] type relative permeabilities in different regions of the saturation space. The approach we use in this report is simpler, and the polynomial form of relative permeability gives good predictions of the recoveries and saturation paths, as we show later. 

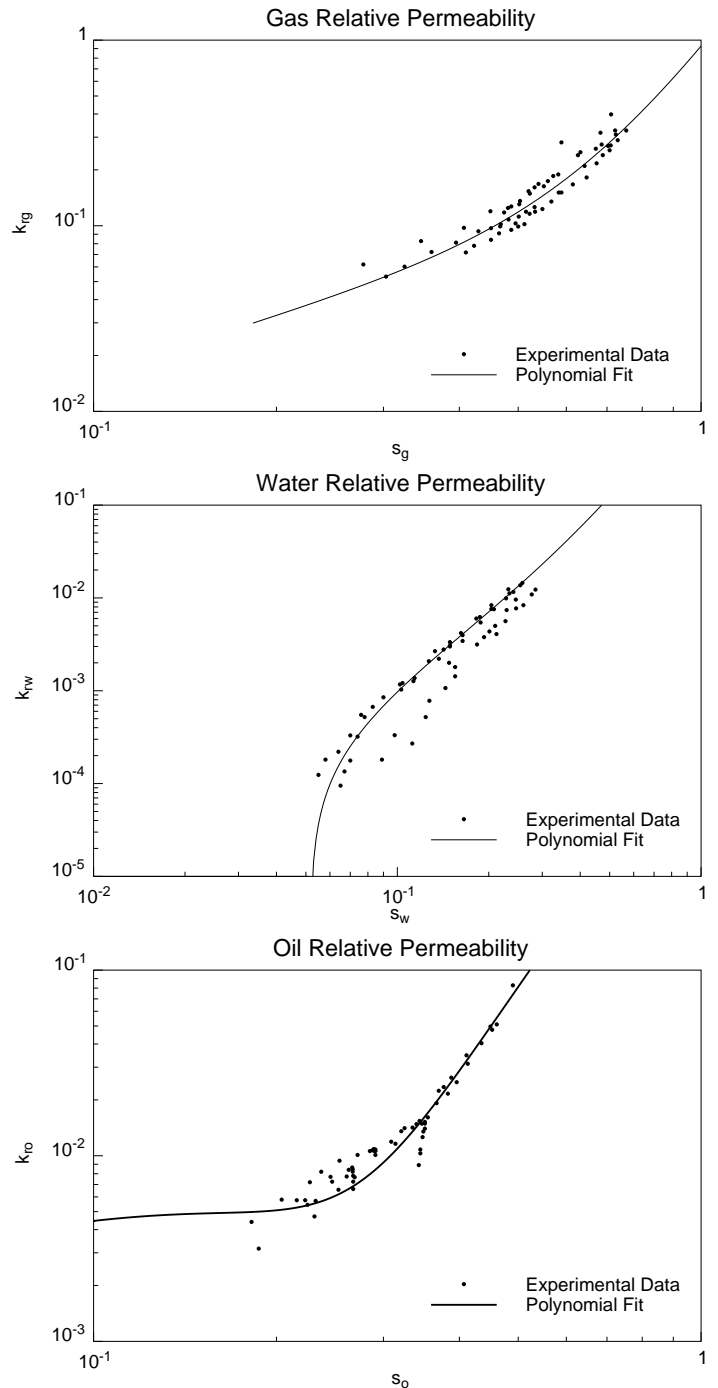

Figure 9.2: Measured and fitted relative permeabilities

Table 9.1: Polynomial fit to relative permeability

\begin{tabular}{|l|l|c|c|c|l|}
\hline$k_{r p}$ & $\mathrm{~A}$ & $\mathrm{~B}$ & $\mathrm{C}$ & $\mathrm{D}$ & $\mathrm{E}$ \\
\hline Oil & 0.079 & -0.399 & 0.436 & 0.878 & 1.023 \\
\hline Gas & 0.160 & -0.009 & 0.091 & 0.261 & 0.425 \\
\hline Water & -0.01 & 0.2 & -0.198 & 1.662 & -0.032 \\
\hline
\end{tabular}


We will now summarize the procedure to calculate the analytical solution given by Guzman and Fayers $[80,81,79]$. The dimensionless conservation equation, in one dimension, ignoring capillary pressure may be written as:

$$
s_{t}+A s_{x}=0
$$

where

$$
\begin{gathered}
s=\left(\begin{array}{c}
s_{w} \\
s_{g}
\end{array}\right) \\
s_{t}=\left(\begin{array}{c}
\frac{\partial s_{w}}{\partial t} \\
\frac{\partial s_{g}}{\partial t}
\end{array}\right) \\
s_{x}=\left(\begin{array}{c}
\frac{\partial s_{w}}{\partial x} \\
\frac{\partial s_{g}}{\partial x}
\end{array}\right)
\end{gathered}
$$

and

$$
A=\left(\begin{array}{ll}
\frac{\partial f_{w}}{\partial s_{w}} & \frac{\partial f_{w}}{\partial s_{g}} \\
\frac{\partial f_{g}}{\partial s_{w}} & \frac{\partial f_{g}}{\partial s_{g}}
\end{array}\right)
$$

$s_{w}$ is the water saturation, $s_{g}$ is the gas saturation, and $f_{w}$ and $f_{g}$ are fractional flows of water and gas respectively. There are two independent saturations, chosen to be $s_{w}$ and $s_{g}$, and the oil saturation is given by:

$$
s_{o}=1-s_{w}-s_{g}
$$

Let $x=0$ be the injection end of the experiment, then we have the initial conditions at time, $t$ $=0: s_{g}=1$ for $x \leq 0$ (we inject gas at $100 \%$ saturation) and $s_{g}=0, s_{w}=s_{w i}$ for $x>0$, where $s_{w i}$ is the initial water saturation in the core. This mathematical statement is a Riemann problem that may be solved by the method of characteristics (MOC). The solutions are functions of $v=$ $x / t$ only. Mathematically, we can write Eq. (2) as:

$$
v \frac{d s}{d v}=A \frac{d s}{d v}
$$

where $v$ is an eigenvalue of $\mathrm{A}$, and $d s / d v$ is an eigenvector, and:

$$
\frac{d s}{d v}=\left(\begin{array}{c}
\frac{d s_{w}}{d v} \\
\frac{d s_{g}}{d v}
\end{array}\right)
$$

The matrix $A$ has two eigenvalues that correspond to characteristic wavespeeds $(v=x / t)$ with which given saturation values will travel.

A rarefaction is a smooth change in saturation with $v$. The eigenvectors of $A$ tell us the path in saturation space taken by a rarefaction as the wavespeed changes. Fig. 9.3 shows these rarefaction curves computed for the polynomial relative permeabilities fit to Grader and O'Meara's 


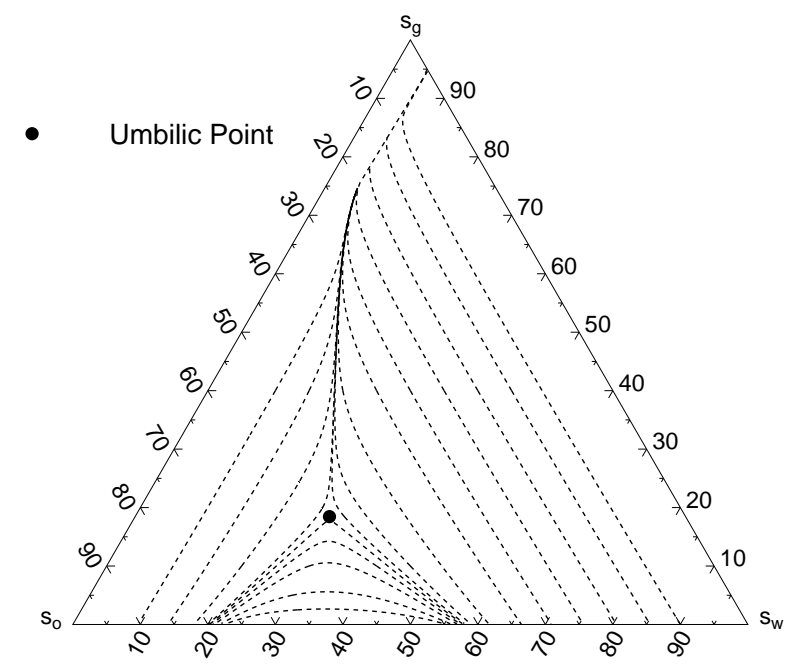

Figure 9.3: Rarefaction curves for the polynomial relative permeabilities fit to Grader and O'Meara's [76] experiments

experiments. Only rarefaction curves for the faster of the two wavespeeds are shown. In the appendix we show how the eigenvalues and eigenvectors are computed, and how the rarefaction curves are found.

An analytical solution is constructed by following a rarefaction curve from the injection condition $\left(s_{g}=1\right.$ in this case) to the initial condition as the wavespeed increases monotonically. The reason the wavespeed has to increase is to avoid multiple-valued solutions for $s(v=x / t)$. For most cases this simple procedure is not possible and a solution can only be constructed with shocks or discontinuities in saturation. Shocks obey an integral form of the conservation equation and the shock speed is:

$$
V_{s h}=\frac{f_{p}^{L}-f_{p}^{R}}{s_{p}^{L}-s_{p}^{R}}
$$

where $L$ and $R$ label the saturations to the left (upstream) and right (downstream) of the shock respectively, and $p=w, g$. Eq. 9.10 is obeyed by both water and gas phases. Constant states, where $s$ is constant as $v$ varies, may separate shocks and rarefaction waves. It is possible to construct a unique path from injection to initial conditions as the wavespeed (or shock speed) monotonically increases, composed of rarefactions, shocks and constant states. How these solutions are constructed for the cases presented in this report is described in the appendix.

If $k_{r p}$ is a function of its own saturation, then $A$ always has real eigenvalues [210]. More complex models that assume at-least one $k_{r p}$ dependent on two saturations (such as Stone's models $[200,201])$ yield complex wavespeeds. Regions in saturation space with complex wavespeeds are called elliptic regions.

If the two eignevalues of $A$ are equal at some $s_{w}$ and $s_{g}$ then we have an umbilic point, and both wavespeeds are the same. Corey type relative permeabilities give a single umbilic point (Holden [95]) and no elliptic regions. If the relative permeabilities are more general functions of their own phase, Guzman and Fayers [80, 81, 79] showed that there may be none, one or multiple umbilic points, and again no elliptic regions. It is the presence of points of inflexion $\left(d^{2} k_{r p} / d s_{p}^{2}=0\right.$, for $s_{p} \neq 0$ and $s_{p} \neq 1$ ) in any of the relative permeability curves that gives rise to the more complex structure. The relative permeabilities we use here do have points of inflexion. There is a single 
umbilic point (at $s_{w}=0.2879$ and $s_{g}=0.184$ ) in the three phase region and the structure of the rarefaction curve is qualitatively similar to that obtained using Corey type relative permeabilities $[60,59]$.

Inspection of the rarefacton curves, Fig. 9.3. gives an indication of the saturation paths. For low oil saturation, the rarefactions are aligned at approximately constant $s_{o}$, which means that gas will preferentially displace water, with little displacement of oil. This makes physical sense for horizontal gas injection at low oil saturation where the water relative permeability is much larger than the oil relative permeability. For large oil saturation, the rarefactions have approximately constant $s_{w}$. This implies that gas preferentially displaces oil since $k_{r o}>k_{r w}$. Notice that there is a dividing rarefaction $[60,59]$ that passes through the umbilic point and separates paths with $s_{o}$ approximately constant from those with $s_{w}$ approximately constant. This dividing curve has a critical influence on the analytical solutions and its precise location is extremely sensitive to the relative permeabilities. For example, we attempted to fit the experimental data in Fig. 9.2 with a third order polynomial $(D=E=0$ in Eq. (1)). The fit was excellent, but the predicted analytical solutions were not matched to the experiment as well as for a fifth order polynomial. On the other hand, the solutions are insensitive to the predicted relative permeabilities at saturations below those measured in the experiment, since here the wavespeeds are very low. Thus in Fig. 9.2 the very steep $k_{r w}$ curve at low $s_{w}$ and the flat $k_{r o}$ curve at low $s_{o}$ which are artifacts of the polynomial fit, have very little effect on the solutions. Also note that the rarefactions shown in Fig. 9.3 appear to start at $s_{g}=0.95$, rather than $s_{g}=1$. This is because the predicted water relative permeability at low saturation is very small and goes to zero for $s_{w}$ approximately $5 \%$. This represents a connate water saturation. The important point to stress here is that a good match to experiments is obtained by a good match to the relative permeabilities for the saturations actually measured; the extrapolation of the relative permeabilities outside the region probed experimentally has no physical meaning.

As a check on our analytical solution, we also performed numerical simulations of the three phase flow problems. We used an explicit, first order in time, second order in space, total variation diminishing (TVD) scheme (Blunt and Rubin [17]) with 800 gridblocks.

Fig. 9.4 shows the saturation path for horizontal gas injection into low oil saturation $\left(s_{w i}=\right.$ $\left.0.696, s_{o i}=0.304\right)$. Grader and O'Meara [76] do not provide sufficient information to construct the saturation profiles for their experiments, but we can compare directly the measured and predicted saturation paths. This case recovers very little incremental oil. The analytical solution follows the rarefaction curve and then shocks directly to the initial conditions. Except for small $v$, the rarefaction and shock are at approximately constant oil saturation. Thus the oil recovery is poor unless a huge number of pore volumes of gas is injected.

Fig. 9.5 shows gas injection into a higher oil saturation $\left(s_{w i}=0.518, s_{o i}=0.482\right)$. Here it is not possible to find a shock from the rarefaction curve to the initial conditions. The analytic solution features a shock from the three phase region to the two phase region, and then a two phase shock to the initial conditions. This solution has an oil bank. Fig. 9.6 for $s_{w i}=0.447, s_{o i}=0.553$, shows qualitatively the same features. The analytical solution for the cases shown in Figs. 9.5 and 9.6 follow the same path in the three phase region.

For all cases, the analytical and experimental paths are similar. The formation of an oil bank, and a three phase shock has been observed directly using in-situ saturation measurements for similar experiments [192]. Our analytical predictions for runs \#15 and \#7 are not shown, but they also agree well with the experiments. The numerical and analytical one-dimensional solutions for saturation are virtually identical.

The polynomial relative permeabilities we used assumed that the residual oil saturation was zero. This implies that given sufficient time, gas could displace all the oil and water from the core. In reality, however, the experiments are run for only a finite time. Since the wavespeeds for drainage at low saturations are very slow (corresponding to low relative permeability), it would take 


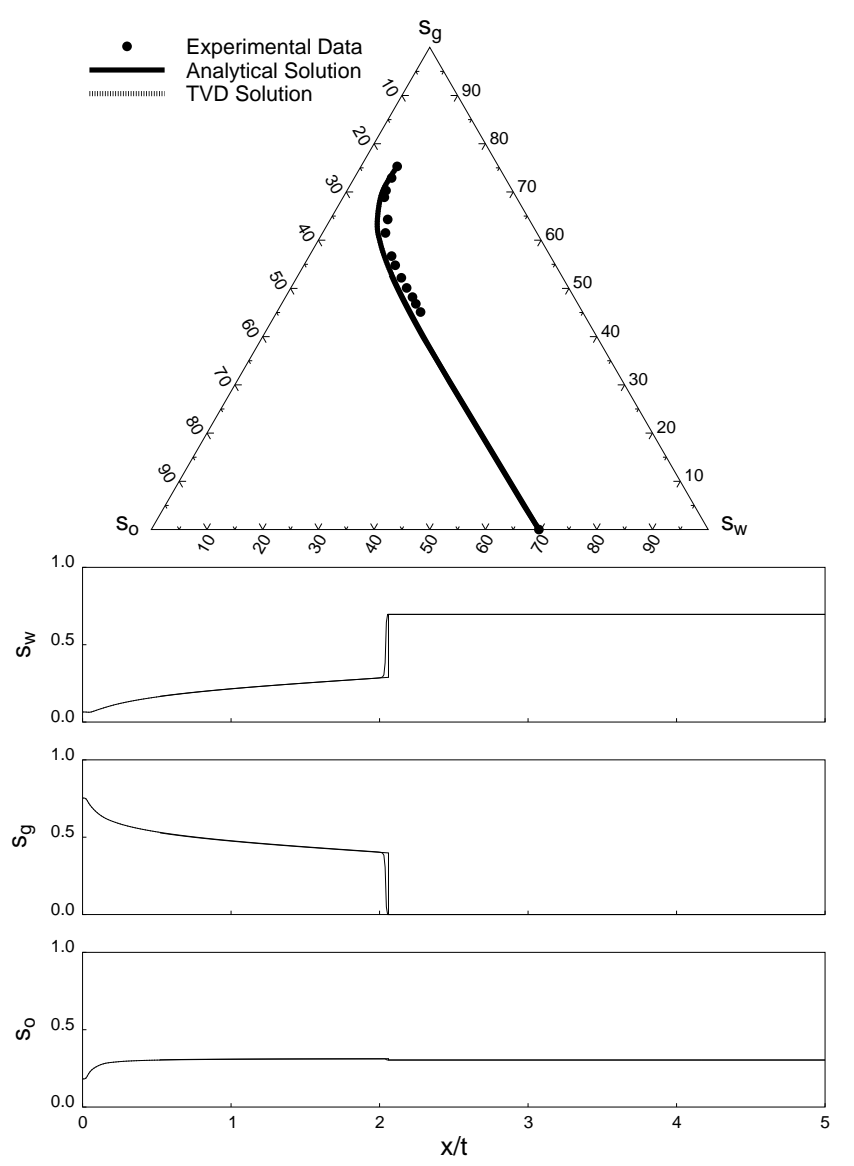

Figure 9.4: Predicted and measured saturation paths for Grader and O'Meara's [76] run \#9 


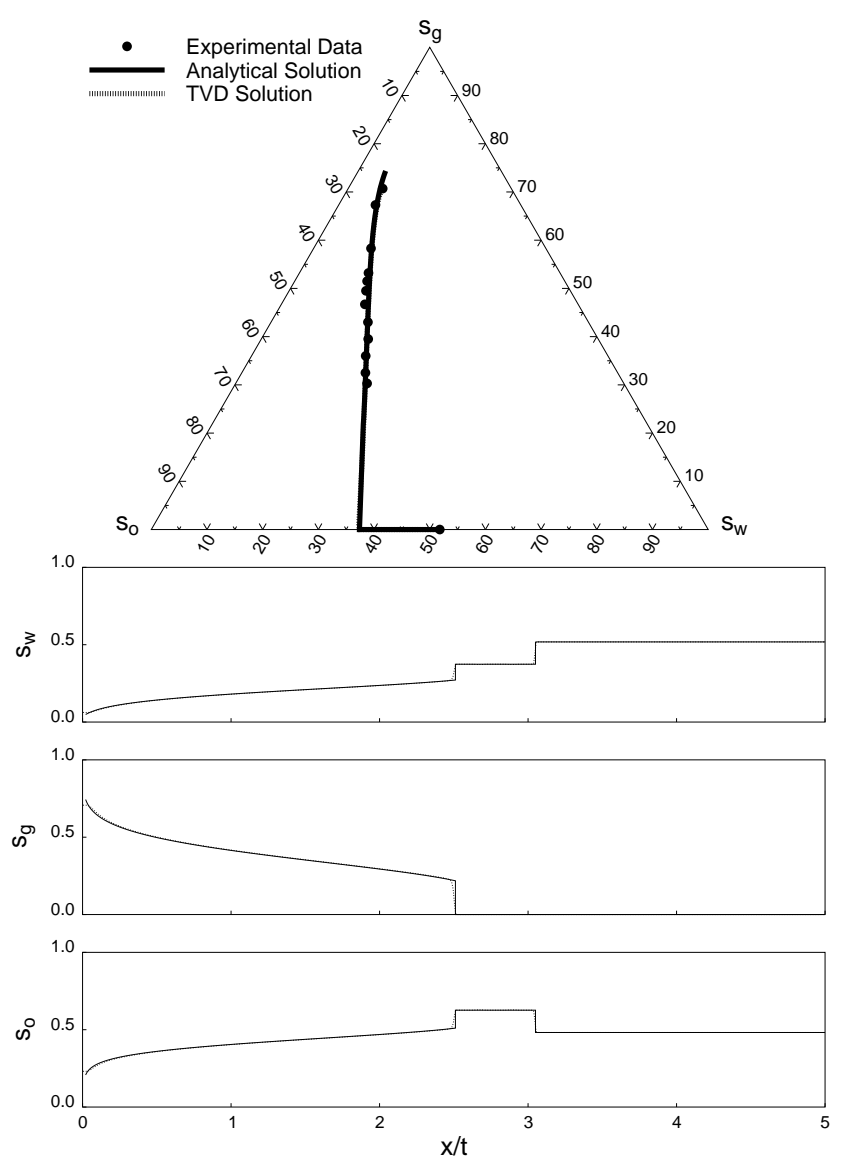

Figure 9.5: Predicted and measured saturation paths for Grader and O'Meara's [76] run \#8 


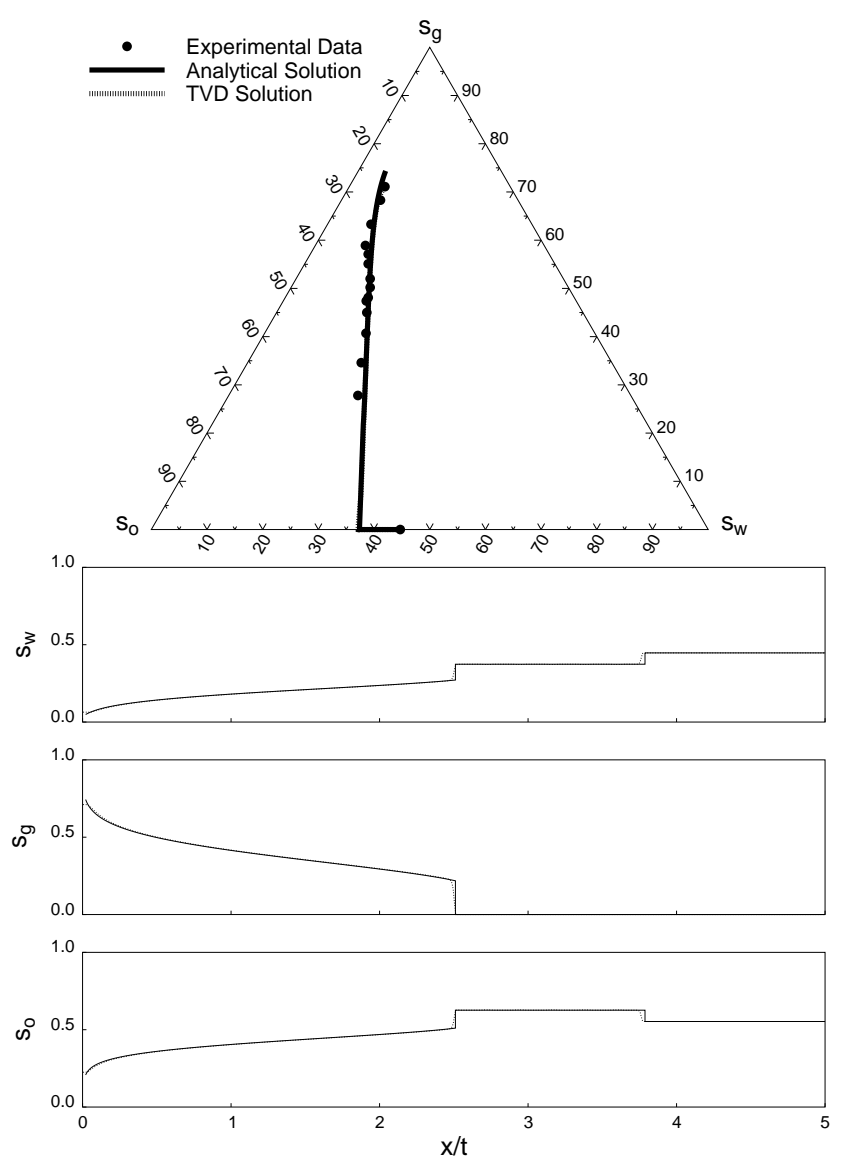

Figure 9.6: Predicted and measured saturation paths for Grader and O’Meara's [76] run \#5 


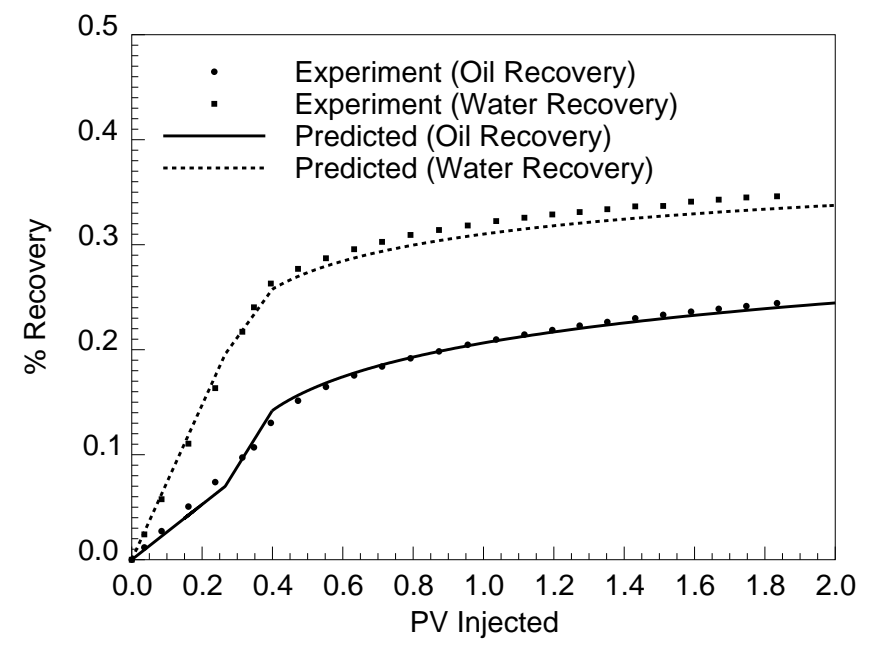

Figure 9.7: Predicted and measured recoveries for Grader and O'Meara's [76] run \#5

huge amounts of gas to displace oil at very low saturations. For the range of saturations actually encountered, the theoretical predictions are excellent. Even if the relative permeabilities predict zero residual oil saturation, this saturation will never be reached in a realistic experiment.

Falls and Schulte $[60,59]$ presented analytical solutions for three phase flow with Corey type relative permeabilities, for which a single umbilic point was observed. The solutions obtained here are similar to the ones obtained by Falls and Schulte, and there is a narrow range of initial conditions for which the solution follows the same path in the three phase region (Figs. 9.5 and 9.6 ). The use of Stone [200, 201] relative permeabilities or different curves for different regions of saturation space, as suggested by Hicks and Grader [89], give elliptic regions. These are unphysical artifacts of the extrapolation of the relative permeabilities outside the domain probed experimentally. The elliptic regions can cause instabilities or may give rise to non-unique solutions [210]. In this work, the experimental data is matched successfully using polynomial relative permeabilities, and there are no elliptic regions.

\section{Recovery Calculation}

Once the saturation profiles have been obtained, the oil recovery can be computed analytically. Fig. 9.7 shows the oil and water recoveries as a function of pore volumes of decane injected for run \#5. The computed recoveries match well with the experimental data. As seen in Fig. 9.7 the increase of slope of the recovery curve for oil just before 0.3 pore volumes injected is due to the breakthrough of the oil bank prior to the gas.

\section{Conclusions}

For the experiments analyzed the three phase relative permeabilities were, to a good approximation, functions of their own saturation. Analytical solutions for the saturation paths and recoveries computed using a polynomial fit to the relative permeabilities predicted the results of the experiments well. The fitted relative permeabilities did not give elliptic regions and there was a single umbilic point in the three phase region. For a narrow range of initial conditions a two phase shock, and an oil bank developed, and the saturation paths in the three phase region were the same. Outside this narrow range, different initial conditions may lead to different saturation paths through the three phase region. The solution structure is similar to that obtained from Corey type relative permeabilities [60, 59]. 


\subsection{Nomenclature}

$A=$ Jacobian matrix of flux vector $f$

$d s=$ right eigenvector of matrix $A$

$f=$ flux vector with components $f_{w}$ and $f_{g}$

$f_{g}, f_{w}=$ gas and water fractional flow

$k=$ absolute permeability

$k_{r p}=$ relative permeability to phase $p$

$\nu=\mathrm{x}$-ray attenuation of material

$s_{p}=$ saturation $(\mathrm{p}=\mathrm{o}, \mathrm{g}, \mathrm{w})$

$s=$ saturation vector with components $s_{w}$ and $s_{g}$

$t=$ dimensionless time (injected pore volumes)

$V_{s h}=$ shock velocity

$v \quad=$ eigenvalues of matrix $A$

$x=$ dimensionless distance

\section{Greek Letters}

$\delta=$ discriminant

$\phi \quad=$ porosity

\section{Subscripts}

$1=$ first energy level

$2=$ second energy level

$g=$ gas

$o \quad=$ oil

$p=$ phase

$t=$ derivative with respect to $t$

$w=$ water

$x=$ derivative with respect to $x$

\section{Superscripts}

$L=$ left state

$R=$ right state

\subsection{Appendix}

More details on the construction of analytical solutions to three phase flow are given in References [80, 81] and [79]. The dimensionless conservation Equation 9.2. is:

$$
s_{t}+A s_{x}=0
$$

where $A$ is given by Equation 9.6. The eigenvalues of $A$ are:

$$
v=\frac{1}{2}\left(f_{w w}+f_{g g}\right) \pm \sqrt{\delta}
$$

where

$$
\delta=\left(f_{w w}-f_{g g}\right)^{2}+4 f_{g w} f_{w g}
$$


and

$$
f_{p q}=\frac{\partial f_{p}}{\partial s_{q}}
$$

The eigenvectors are aligned along the direction

$$
d s=\left(\begin{array}{c}
1 \\
\frac{d s_{g}}{d s_{w}}
\end{array}\right)=\left(\begin{array}{c}
1 \\
\left(f_{g g}-f_{w w} \pm \sqrt{\delta}\right) / 2 f_{w g}
\end{array}\right)
$$

and thus the ratio of a change in $s_{g}$ to $s_{w}$ along an eigenvector is:

$$
d s_{g}=\left(\left(f_{g g}-f_{w w} \pm \sqrt{\delta}\right) / 2 f_{w g}\right) d s_{w}
$$

A rarefaction curve is found by starting at some given $s=\left(s_{w}, s_{g}\right)$ and using Equation 9.16. to find the change in $s_{g}$ for a small change in $s_{w}$. Then Equation 9.16. is recomputed and the new eigenvalues are found. In this way a curve in $\left(s_{w}, s_{g}\right)$ space is traced. In Figure 9.3, the faster rarefaction is found, i.e., the positive root in Equation 9.16. is taken.

To construct the analytical solutions we start at the initial condition and attempt to find a shock into the three phase region, where

$$
v\left(s_{p}^{L}\right)=V_{s h}=\frac{f_{p}^{L}-f_{p}^{R}}{s_{p}^{L}-s_{p}^{R}}
$$

for $p=w, g . s_{p}^{R}=s_{p i}$, i.e. the initial condition and we try to find $s_{p}^{L}$ that obeys these conditions. Equation 9.17 represents two independent equations for two unknowns $\left(s_{w}^{L}, s_{g}^{L}\right)$. Once $s_{w}^{L}$ and $s_{g}^{L}$ are known we follow a rarefaction curve back to the injection conditions. Note that Equation 9.17. describes a Buckley-Leverett type shock, where the shock speed is equal to the wavespeed of the left state.

For Figures 9.5 and 9.6 we could not find a shock from the initial conditions to the three phase region. For three phase flow with a unique umbilic point, the three phase shock jumps from the two phase region to the dividing rarefaction that passes through the umbilic point [60, 59]. We have three unknowns, $s_{w}^{L}, s_{g}^{L}$ and $s_{w}^{R}$, the water saturation in the two phase region. Equation 9.17 gives two indepedent equations. Insisting that $s_{w}^{L}$ and $s_{g}^{L}$ lie on the rarefaction that passes through the umbilic point gives the third equation. To aid in the solution of these equations we can use the numerical solution as an initial guess for $s_{w}^{R}$. There is then a two phase shock to the initial conditions. This shock speed must be larger than for the three phase shock. 


\title{
10. Measurement of Three Phase Relative Permeability during Gravity Drainage using CT Scanning
}

\author{
Akshay Sahni, Jon Burger, and Martin Blunt
}

We measured three phase relative permeabilities for gravity drainage using a dual-energy medical CT scanner modified to scan vertical cores. Independent measurements of two saturations as a function of time and distance along the length of the core were made from which relative permeabilities were found. Three phase (air/oil/water) gravity drainage experiments were performed on systems with different spreading coefficients and at different initial conditions. Experiments were run on both consolidated and unconsolidated porous media. The results were compared to measurements of three phase flow in capillary tubes, micromodels and to predictions from network modeling.

We find that at low oil saturation $k_{r o} \sim S_{o}^{2}$ for hexane and octane as the oil phase. This functional form of relative permeability is consistent with the drainage of oil layers, wedged between the water and gas in crevices of the pore space. For decane, which is non-spreading, the layer drainage regime was not observed. At higher oil saturations $k_{r o} \sim S_{o}^{a}$ with $a \approx 4$ for spreading and non-spreading systems. Within the scatter of the experimental data, oil and water relative permeability are functions only of their own saturations and independent of initial conditions.

\subsection{Introduction}

The simultaneous flow of three immiscible fluids in porous media is an essential component of enhanced oil recovery and aquifer remediation processes. Three phase flow may occur during solution gas drive, gas injection, surfactant flooding and thermal recovery. High oil recoveries have been observed during three phase gravity drainage when gas displaces oil downdip in the presence of water. $[49,114,113,227,19]$ Three phase gravity drainage is of importance in many oilfields under tertiary recovery, such as Prudhoe Bay.[103, 57]

Three phase gravity drainage is also an important process in the environmental industry. Leakage of non-aqueous phase liquid (NAPL) is a major source of groundwater contamination. The displacement of NAPL in the presence of air and water is similar to the process of gravity drainage in oil reservoirs. For designing efficient subsurface cleanup processes it is essential to determine the amount of trapped NAPL and the rate at which the NAPL migrates downwards to the water table.

In recent years, experiments in micromodels[115, 196, 158, 30, 160] and capillary tubes[229, 47] have demonstrated typical three phase configurations at the pore scale, together with pore-level displacement processes and representative flow rates for oil and water in a single pore. Using this knowledge of the behavior at the pore scale, several three phase network models have been developed which have computed three phase relative permeabilities and oil recoveries.[68, 69, 195, $131,162,84,178]$

While research at the pore scale provides a good conceptual picture of several three phase flow phenomena, carefully characterized experiments at the core scale are necessary to validate the macroscopic predictions of pore level models, and to provide a basis for predicting and interpreting three phase flow at the field scale.

\footnotetext{
${ }^{1}$ The material in this chapter was presented at the 1998 SPE/DOE Improved Oil Recovery Symposium in Tulsa, Oklahoma, April 19-22, 1998, and is published as SPE paper 39655 in the conference proceedings.
} 
The following sections discuss some of the published three phase experiments and relative permeability estimation techniques. Then we give an outline of our experimental approach.

\subsubsection{Gravity Drainage Experiments}

Vizika and Lombard[217] studied the effects of wettability and spreading characteristics of the fluid system in three phase gravity drainage. The highest oil recoveries were obtained for spreading systems and water-wet or fractionally-wet conditions. Slow oil production after gas breakthrough was observed for spreading systems, which resulted, eventually, in low oil saturations. For nonspreading systems, oil recoveries were lower. The lowest oil recoveries were obtained in oil-wet porous media as the oil was subject to strong capillary retention. The relative permeabilities for the experiments were obtained numerically by history matching the oil production data. In waterwet and in fractionally-wet porous media the oil relative permeability was higher for spreading systems than for non-spreading systems.

Skurdal et al.[194] analyzed gravity drainage experiments using spreading and non-spreading systems under oil-wet, water-wet and mixed-wet conditions. The final oil saturations obtained from all the experiments varied from $3 \%$ to $8 \%$. The results did not allow firm conclusions to be drawn regarding the effect of spreading characteristics on oil recovery. For the experiments conducted the impact of wettability on oil recovery was small.

Naylor et al.[147] performed gravity drainage experiments by measuring in-situ oil and brine saturations using a radioactive tracer technique. Analysis showed that the oil relative permeability was a function only of the oil saturation and had a zero asymptotic residual oil saturation. The experiments were conducted on a consolidated Clashach sandstone with a spreading oil.

Skauge et al.[193] summarized results from gas gravity drainage experiments at different water saturations for oil having a spreading coefficient of zero. In-situ measurements of saturation were obtained using a nuclear tracer technique. It was observed that increasing the water saturation changed the oil recovery by gravity drainage under water-wet conditions. The oil recovery was higher with connate water present than without, with the maximum recovery being at an initial water saturation of $30 \%$.

\subsubsection{Calculating Three Phase Relative Permeability}

Virnovsky[216] and Grader and O'Meara[76] developed a theory to obtain three phase relative permeability by an extension of the Welge[222] and JBN[111] methods to three phases. Siddiqui et al. [192] verified the theory using X-ray computerized tomography to obtain in-situ saturations for three phase dynamic displacement experiments. Sarem[189] obtained three phase relative permeability by unsteady state displacement experiments assuming that relative permeability of each phase was a function of its own saturation. Oak et al.[155] presented a steady-state study of three phase relative permeability using fired Berea cores. Naylor et al.[147] obtained three phase relative

permeability from gravity drainage experiments using in-situ saturation measurements and ignoring capillary pressure. Nordvedt et al.[152] performed displacement experiments on low-permeability chalk samples and obtained three phase relative permeability from an optimization technique using a general purpose three phase simulator. Akin and Demiral[2] obtained three phase relative permeability from unsteady state displacement experiments using an automated history matching technique.

Despite the large body of experimental three phase relative permeabilities, most numerical models of three phase flow in porous media use empirical relationships for capillary pressure and relative permeability $[64,168,200,201,211,36]$ that are based on extrapolation of two phase data. Baker[6], Delshad and Pope[40], Oak[155] and Fayers and Matthews[65] compared empirical models 
to published experimental data and showed that in most cases the empirical models fail to match the measurements.

\subsection{Our Approach to the Three Phase Flow Problem}

\subsubsection{Three Phase Gravity Drainage}

We use CT scanning at two different X-ray energies to measure two independent saturations as a function of distance and time during a displacement experiment. From this and the capillary pressure it is possible to obtain a direct measurement of three phase relative permeability. This approach overcomes some of the limitations of previous approaches; namely ignoring capillary pressure[147, 76, 192] or using a possibly non-unique history matching process to obtain relative permeabilities. [217, 2]

While previous work has shown that three phase gravity drainage in water wet porous media leads to low oil saturations through the slow drainage of oil layers in the pore space,[49, 114, 113, 227] there are still several unresolved issues, including:

1. The typical functional form of the oil relative permeability, particularly at low saturation.

2. The effect of spreading coefficient on relative permeabilitiy. While some experiments have shown that spreading coefficient has a large effect on the recovery,[217] others have shown little variation. $[49,194]$

Recent theoretical work based on consideration of three phase flow at the pore scale has lead to the following predictions which attempt to answer the points above:[229, 47, 68]

1. That at low oil saturation, where flow is controlled by layer drainage, $k_{r_{o}} \sim S_{o}{ }^{2}$. At higher saturation $k_{r_{o}}$ is governed by the network of pores filled with oil and $k_{r o} \sim S_{o}{ }^{a}$, with an exponent $a \approx 4$.

2. That layer drainage is possible for both spreading and non-spreading systems, but that the range of capillary pressures over which layers may form decreases with decreasing spreading coefficient. This means that for a non-spreading oil, the layer drainage regime may be absent. At higher saturations $k_{r o}$ is insensitive to spreading coefficient.

We performed a series of three phase gravity drainage experiments under different spreading and initial conditions, in water-wet consolidated and unconsolidated media in order to test these theoretical predictions. To obtain different spreading conditions we use three different oils; nhexane, n-octane and n-decane. Table 10.1 gives a list of the experiments performed.

\subsubsection{Analysis of Gravity Drainage Experiments}

Equations to find the relative permeability during gravity drainage can be obtained from a rearrangement of the multi-phase Darcy law, the capillary pressure equations and the conservation equation for each phase. We develop here equations needed to compute relative permeability during a three phase gravity drainage experiment.

For three phase flow we can write Darcy's law for each of the three phases as

$$
u_{w}=-\frac{k k_{r w}}{\mu_{w}}\left(\frac{\partial p_{w}}{\partial z}-\rho_{w} g\right)
$$


Table 10.1: Three Phase Gravity Drainage Experiments. $S_{o i}$ is the initial oil saturation averaged over the whole core. $S_{\text {of }}$ is the final oil saturation at the end of the experiment averaged over the top half of the column (top $45 \mathrm{~cm}$ ). $S_{w f}$ is the final average water saturation in the top half of the column. The $*$ indicates waterflood residual oil $\left(S_{\text {or }}\right)$

\begin{tabular}{|c|c|c|c|c|c|}
\hline Expt. & Oil & Medium & $S_{o i}$ & $S_{o f}$ & $S_{w f}$ \\
\hline 1 & n-octane & sand & 0.81 & 0.012 & 0.11 \\
\hline 2 & n-octane & sand & $0.13^{*}$ & 0.01 & 0.16 \\
\hline 3 & n-octane & sand & 0.15 & 0.021 & 0.16 \\
\hline 4 & n-hexane & sand & 0.80 & 0.013 & 0.21 \\
\hline 5 & n-decane & sand & 0.85 & 0.124 & 0.18 \\
\hline 6 & n-octane & sandstone & $0.30^{*}$ & 0.189 & 0.51 \\
\hline
\end{tabular}

$$
\begin{aligned}
& u_{o}=-\frac{k k_{r o}}{\mu_{o}}\left(\frac{\partial p_{o}}{\partial z}-\rho_{o} g\right) \\
& u_{g}=-\frac{k k_{r g}}{\mu_{g}}\left(\frac{\partial p_{g}}{\partial z}-\rho_{g} g\right)
\end{aligned}
$$

By definition $z=0$ is the top of the column and $z$ increases downwards.

In our experiments, air is the gas phase, which flows very slowly. Thus to a very good approximation there is no potential gradient in the gas phase. Thus,

$$
\frac{\partial p_{g}}{\partial z}=\rho_{g} g
$$

or

$$
p_{g}=\rho_{g} g z
$$

where we define $p_{g}=0$ at $z=0$.

We can write the capillary pressure equations as

$$
\begin{aligned}
& p_{g}-p_{o}=p_{c g o} \\
& p_{o}-p_{w}=p_{c o w}
\end{aligned}
$$

Therefore

$$
u_{o}=\frac{k k_{r o}}{\mu_{o}}\left(\frac{\partial p_{c g o}}{\partial z}+\Delta \rho_{g o} g\right)
$$




$$
u_{w}=\frac{k k_{r w}}{\mu_{w}}\left(\frac{\partial p_{c o w}}{\partial z}+\frac{\partial p_{c g o}}{\partial z}+\Delta \rho_{w g} g\right)
$$

where

$$
\Delta \rho_{g o}=\rho_{o}-\rho_{g}
$$

and

$$
\Delta \rho_{w g}=\rho_{w}-\rho_{g}
$$

Conservation of water implies

$$
\phi \frac{\partial S_{w}}{\partial t}+\frac{\partial u_{w}}{\partial x}=0
$$

Hence

$$
\left.u_{w}\right|_{z}=-\int_{0}^{z} \phi \frac{\partial S_{w}}{\partial t} d z
$$

where the integration is taken from the top of the column $(z=0)$.

Conservation of oil implies

$$
\phi \frac{\partial S_{w}}{\partial t}+\frac{\partial u_{o}}{\partial x}=0
$$

Hence

$$
\left.u_{o}\right|_{z}=-\int_{0}^{z} \phi \frac{\partial S_{o}}{\partial t} d z
$$

Thus the equations for the water and oil relative permeabilities are from Eqs. 10.8, 10.9, 10.13 and 10.15 .

$$
\begin{gathered}
k_{r o}=-\frac{\mu_{o} \phi}{k} \frac{\int_{0}^{z} \frac{\partial S_{o}}{\partial t} d z}{\left(\frac{\partial p_{c g o}}{\partial z}+\Delta \rho_{g o} g\right)} \\
k_{r w}=-\frac{\mu_{w} \phi}{k} \frac{\int_{0}^{z} \frac{\partial S_{w}}{\partial t} d z}{\left(\frac{\partial p_{c o w}}{\partial z}+\frac{\partial p_{c g o}}{\partial z}+\Delta \rho_{o w} g\right)}
\end{gathered}
$$

For a two phase, air/water experiment, a similar derivation gives

$$
k_{r w}=-\frac{\mu_{w} \phi}{k} \frac{\int_{0}^{z} \frac{\partial S_{w}}{\partial t} d z}{\left(\frac{\partial p_{c g w}}{\partial z}+\Delta \rho_{g w} g\right)}
$$

To calculate the relative permeabilities, we need in-situ saturation measurements as a function of time and distance along the core. We also have to measure the capillary pressure. 

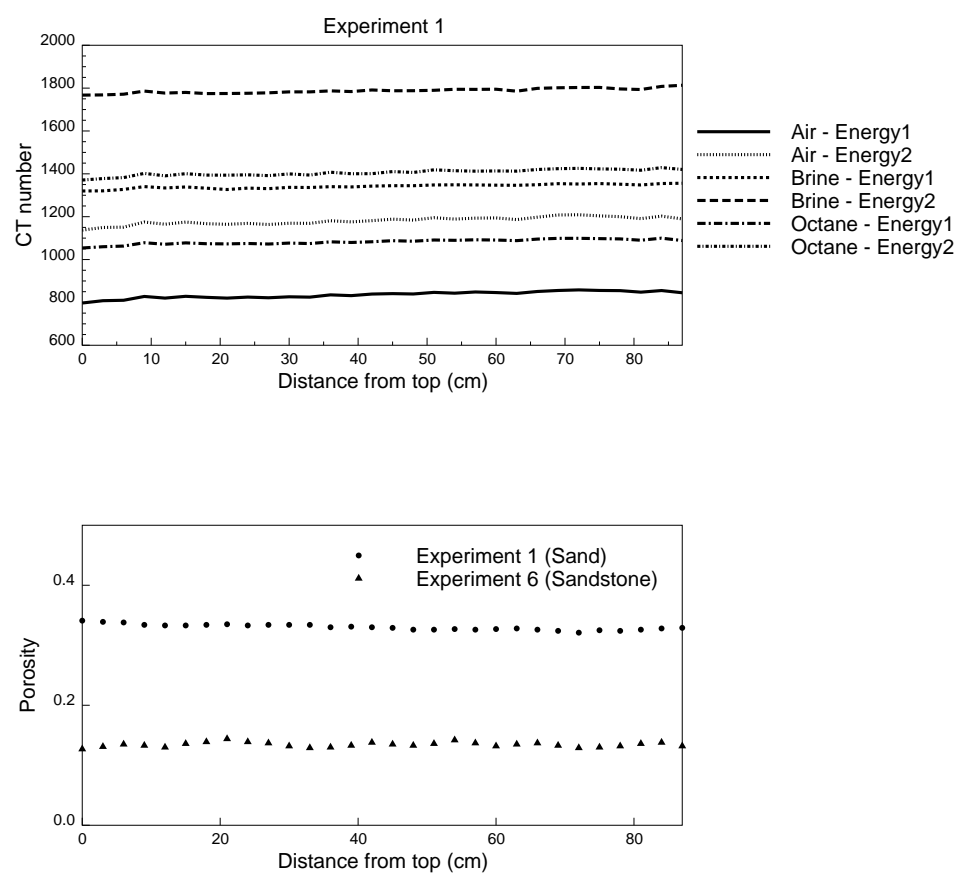

Figure 10.1: Calibration and porosity measurements from the CT Scanner.

\subsection{Dual Energy CT scanning and Experimental Design}

CT scanning is a widely used tool in the petroleum industry[97, 224, 89] and has successfully measured saturation distributions in porous media. There are three methods that have been used to monitor three phase flow experiments in porous media using CT scanning. The first technique assumes that one of the three phases is immobile, and the calculations are the same as the two phase calculations. MacAllister et al.[130] used this method to obtain quantitative estimates of oil, water and gas saturation distributions, assuming the oil phase to be immobile. The oil saturation was estimated by an independent measurement. The authors used two phase CT scans to determine residual oil saturations, in presence of water and gas.

Another way of analyzing three phase flow experiments using CT scanning involves matching the CT numbers of two fluids and treating them as a single phase. Siddiqui et al.[192] used this method to analyze three phase flow experiments. Water, benzyl alcohol and decane were used as the three immiscible phases in the experiment. Water and decane were tagged with iodine to match their CT response, and this allowed the determination of benzyl alcohol saturation with a single high energy scan. However, using this technique it is not possible to separate the water and decane saturations.

The third approach, which we used, is to scan at two different energies to obtain three phase saturations. We used a Picker 1200X fourth generation medical CT scanner with 1200 stationary detectors. The CT scanner was modified to rotate a complete 90 degrees to scan both horizontal and vertical experiments. A remotely operated vertical positioning system mounted on the scanner allowed the vertical movement of the object in the scan frame to an accuracy of $1 / 100$ th of a mm.

At the same location two scans were taken at two different energy levels. The lower energy scan was at $80 \mathrm{kV}$ and the higher energy scan was at $140 \mathrm{kV}$. We scanned the core dry, fully saturated with brine (water phase) and fully saturated with oil at the two energy levels. The brine was a $10 \%$ by weight solution of sodium bromide. Table 10.3 shows the properties of the fluids used. From the dry core scans and $100 \%$ brine saturated scans, we obtained the porosity along the length of the core using Eq. 10.33. 


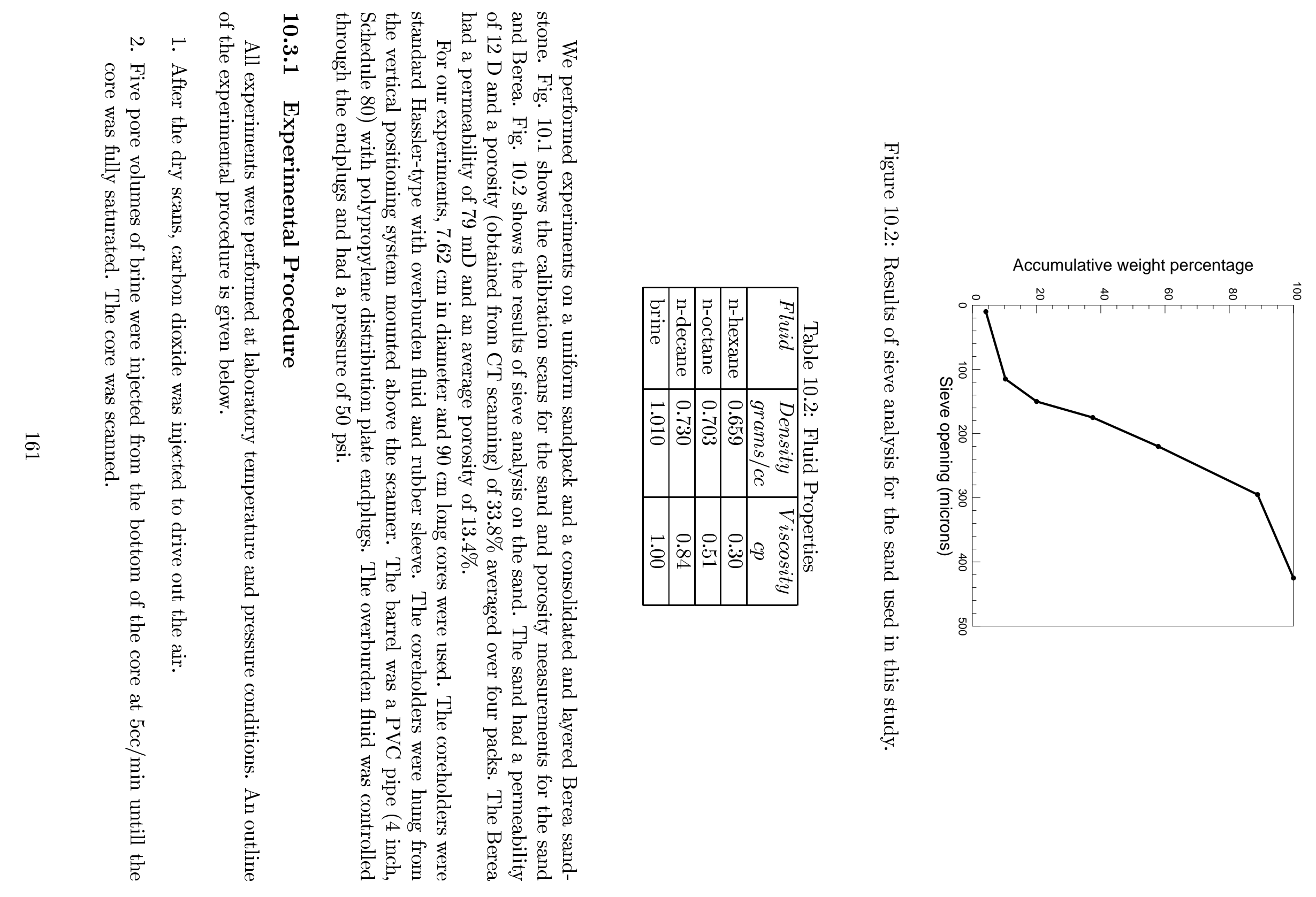


3. Isopropyl alcohol was injected from the top of the column. Isopropyl alcohol is a solvent which dissolves both water and oil.

4. Oil was injected from the top until the core was fully saturated with oil. The core was scanned.

5. The core was cleaned with isopropyl alcohol.

6. Brine was injected to make the core water-wet. All experiments are performed under waterwet conditions.

7. Oil was injected until connate water saturation was reached. This was the initial condition in experiments 1, 4 and 5. In experiments 2, 3 and 6 , brine was then injected to establish initial conditions.

8. The core drained freely under gravity by allowing air to enter from the top. To reduce the vaporization of the fluids in the porous medium, the air entering the core was passed through oil and water, kept in separate containers.

9. The core was scanned during the gravity drainage to obtain in-situ measurements of phase saturations as a function of time and distance along the length of the core. At a given time, the core was scanned every $3 \mathrm{~cm}$ along its length.

\subsubsection{Analog Experiments}

Precise measurements of capillary pressure were obtained from analog experiments performed on the same sand and with the same fluids, in glass columns approximately 1 meter in length and $2 \mathrm{~cm}$ in diameter. The procedure for the analog experiments is similar to that for the CT experiments. These experiments could not be performed on Berea. How we obtained capillary pressure from Berea is described at the end of this section.

1. After weighing the pack, carbon dioxide was injected to drive out the air.

2. This was followed by injecting brine as in the CT experiments.

3. The column and stand were weighed again. The average porosity of the sandpack was obtained from the volume of brine in the column. The average porosity value for the sand column was $34.2 \%$. This is close to the porosity obtained from CT scanning (33.8\%).

4. Oil was injected from the top of the column at $2 \mathrm{cc} / \mathrm{min}$ till no further brine was eluted. Three analog experiments were performed with hexane, octane and decane as the oil phase. A two phase experiment was performed with brine as the liquid phase.

5. The sand column was let to drain freely under gravity by letting in air from the top. After 7 weeks, when we assumed that the fluids were no longer flowing, sand was taken out in $3 \mathrm{~cm}$ intervals along the length of the column. The extracted sand and fluids were washed with 50 $\mathrm{cm}^{3}$ of iso-proponal, a solvent that dissolves both oil and water.

6. The samples were run through a gas chromatograph (HP 5880A series machine) which was calibrated by known volumes of fluids mixed in the solvent.

7. We assume that the sand column had a uniform porosity. Thus we can estimate the total pore volumes of each sample of sand. From the gas chromatograph we obtained the saturation of each phase. 
8. At equilibrium the pressure in the fluids was the gravitational head, and capillary pressures were obtained as follows:

$$
p_{\text {cow }}\left(s_{w}\right)=-\left(\rho_{w}-\rho_{o}\right) g z+\text { constant }
$$

and

$$
p_{c g o}\left(s_{o}+s_{w}\right)=-\left(\rho_{o}-\rho_{g}\right) g z+\text { constant }
$$

We tabulated the results to find $p_{c g o}$ and $p_{c o w}$ for any value of $s_{o}$ and $\left(s_{o}+s_{w}\right)$.

$$
\frac{\partial p_{\text {cow }}}{\partial z}=\frac{p_{\text {cow }}\left(s_{w} \mid z\right)-p_{\text {cow }}\left(\left.s_{w}\right|_{z+}\right)}{\Delta z}
$$

where $z+$ is the distance to the next scan location and $\Delta z=3 \mathrm{~cm}$, is the distance between two scan locations.

Similarly we can find $\frac{\partial p_{c g o}}{\partial z}$ and use the partial derivatives of capillary pressures in Eqs. 10.16 and 10.17 to obtain $k_{r o}$ and $k_{r w}$.

In a two phase air/water experiment we find

$$
\frac{\partial p_{c g w}}{\partial z}=\frac{p_{c g w}\left(\left.s_{w}\right|_{z}\right)-p_{c g w}\left(\left.s_{w}\right|_{z+}\right)}{\Delta z}
$$

And this is used in Eq. 10.18 to find $k_{r w}$.

This procedure cannot be used for consolidated rock. In this case the capillary pressure was obtained from the saturation distribution measured by CT scanning, at the end of the experiment, after 1600 hours of drainage. We assumed that after 1600 hours (9.5 weeks) the fluids were no longer flowing.

\subsubsection{Measuring the Spreading Coefficient}

The spreading coefficient is defined as:

$$
C_{s}=\gamma_{g w}-\gamma_{o w}-\gamma_{g o}
$$

We measured the interfacial tensions $\gamma_{g w}, \gamma_{o w}, \gamma_{g o}$ by the Drop Weight Method.[1] The fluids used were the same as those in the experiments. Strictly speaking we measure the initial spreading coefficient - that is the value before the three fluids obtain thermodynamic equilibrium. If $C_{s}>0$, the oil will spread on a flat water surface, while for $C_{s}<0$ it will not. However, the configuration of three phases at the pore scale is governed by the equilibrium spreading coefficient, $C_{s}{ }^{e}$, where $\gamma_{g w}$ may be lowered due to the presence of a molecular oil film $\left(C_{s}{ }^{e} \leq 0\right)$. [19, 68, 1]

The oil/brine and oil/air interfacial tensions are given in Table 10.3. The measured brine/air interfacial tension is $72 \mathrm{mNm}^{-1}$. The spreading coefficient is computed using Equation 10.23. 
Table 10.3: Interfacial tensions and spreading coefficient

\begin{tabular}{|l|l|c|c|}
\hline Fluid & $\begin{array}{l}\gamma_{\text {ow }} \\
\left(\mathrm{mNm}^{-1}\right)\end{array}$ & $\begin{array}{c}\gamma_{g o} \\
\left(\mathrm{mNm} \mathrm{m}^{-1}\right)\end{array}$ & $\begin{array}{c}C_{s} \\
\left(\mathrm{mNm} \mathrm{m}^{-1}\right)\end{array}$ \\
\hline n-hexane & 50.5 & 18.0 & 3.5 \\
\hline n-octane & 51.2 & 21.1 & -0.3 \\
\hline n-decane & 51.4 & 23.7 & -3.1 \\
\hline
\end{tabular}
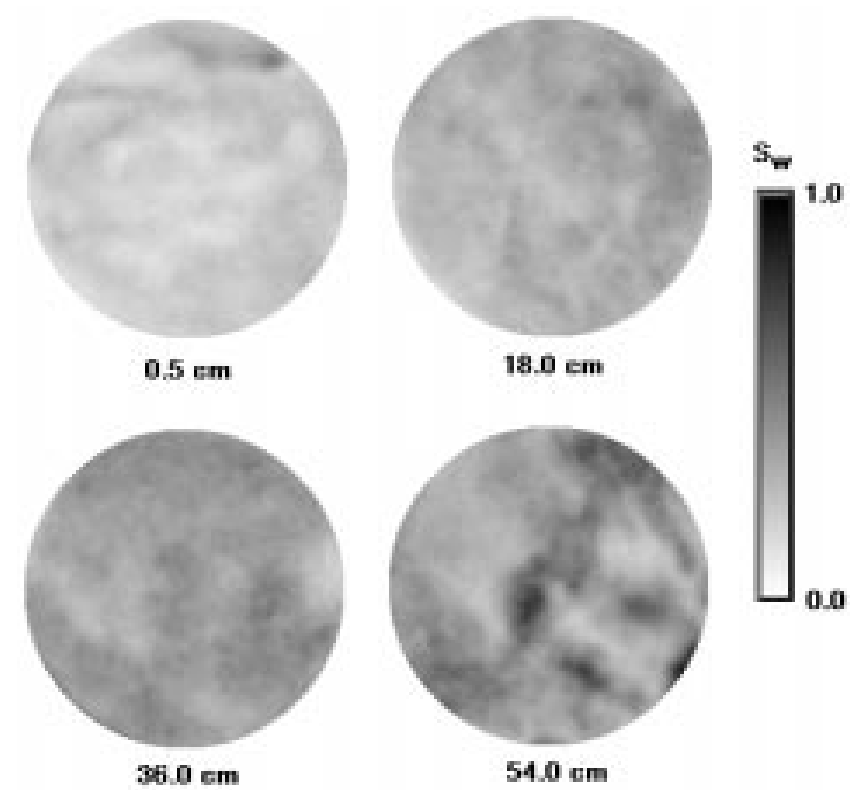

Figure 10.3: In-situ saturation measurements at different locations along the length of the core after 2.50 hours of drainage.

\subsubsection{In-situ Saturation Measurements from Scanned Images}

In this section we show how saturation measurements were obtained by example of a two phase air-water gravity drainage experiment. A fully water saturated sandpack drained freely under gravity and was scanned for a period of 117 hours. The drainage rates were higher at the beginning, so the sandpack was scanned frequently at the start of drainage. CT images of the saturation distribution for different slices along the length of the sand column after 2.5 hours of drainage are shown in Fig. 10.3. The appendix shows how the saturations were obtained from the CT numbers and provides an error analysis.

The water and air saturation profiles were obtained by averaging the saturations at each scan location. Averaging saturation over each slice in Fig. 10.3 gave one data point for the saturation profle. The water and air saturation profiles as a function of time and distance along the length of the core are given in Fig. 10.4. The air/water capillary pressure was obtained by an analog two phase air-water gravity drainage experiment. The relative permeability was found from Eq. 10.18 and is shown in Fig. 10.5. Each saturation measurement gave one relative permeability data point in Fig. 10.5. The three phase relative permeability was obtained in a similar way. 

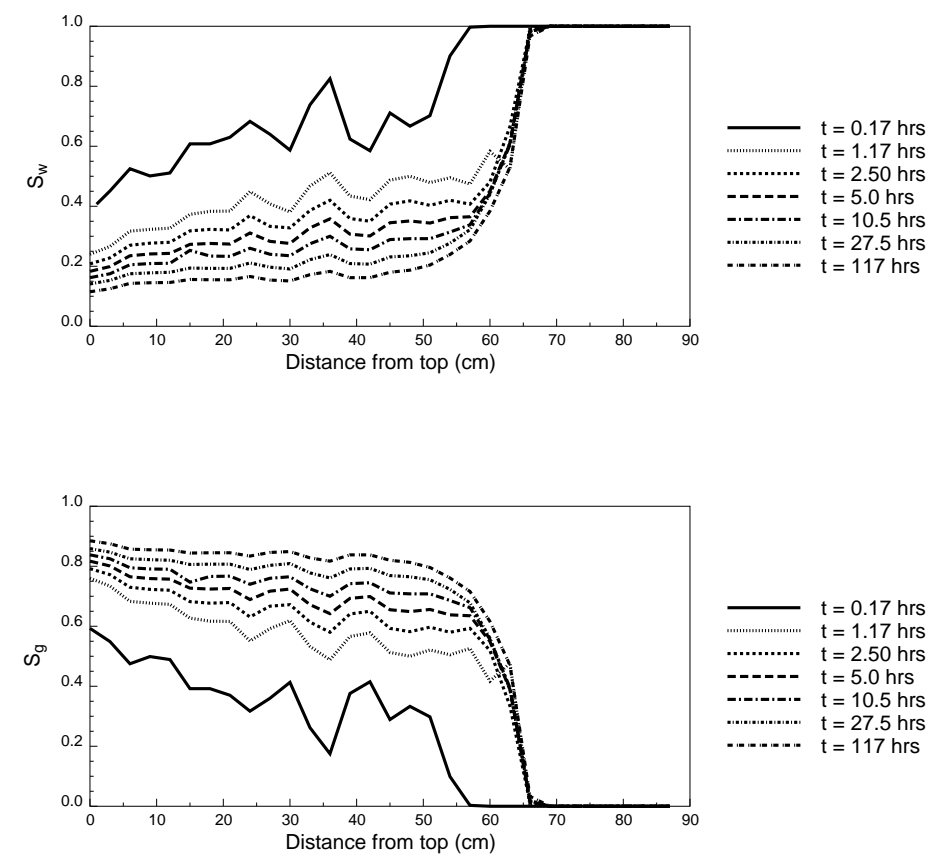

Figure 10.4: Water and air saturation profiles as a function of time and distance along the length of the core.

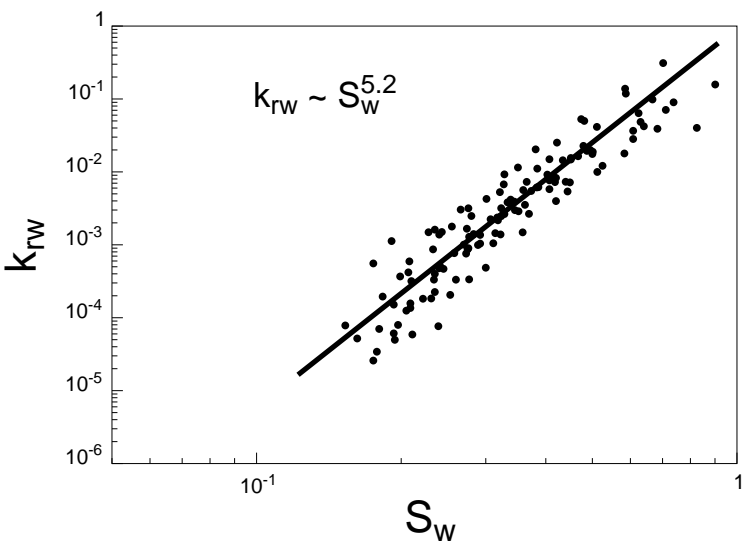

Figure 10.5: Water relative permeability as a function of water saturation. The straight line is a best fit through the data on the doubly logarithmic axes. 

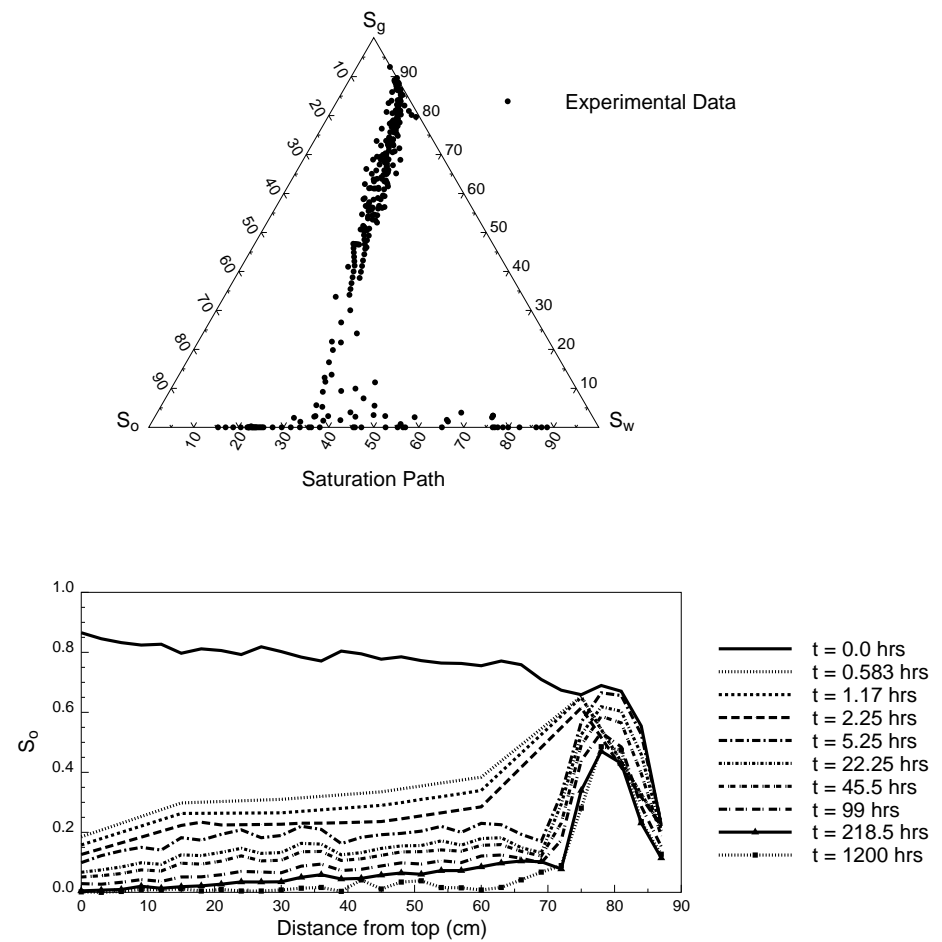

Figure 10.6: Saturation path and oil saturation profile for experiment 1.
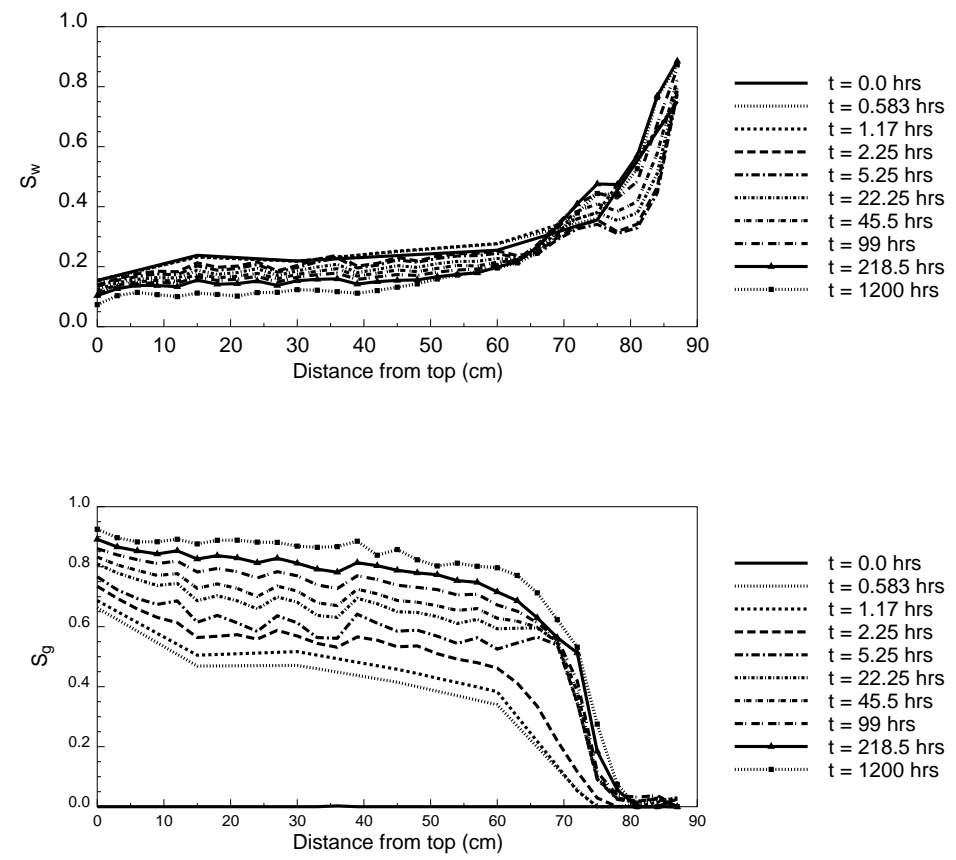

Figure 10.7: Water and air saturation profiles as a function of time and distance for experiment 1. 


\subsection{Three Phase Gravity Drainage Experiments}

Table 10.1 shows the initial and final oil saturations for the experiments performed. Figures 10.6 and 10.7 show the saturation path and saturation profiles for experiment 1 where the oil was octane and $S_{o i}=0.81$. Experiment 2 was similar except that the initial oil saturation represents waterflood residual oil. Three phase gravity drainage displaced almost all of the waterflood residual oil. Experiment 3 used octane with $S_{o i}=0.15$. Experiment 4 used hexane while experiment 5 used decane.

Experiment 6 was performed on a consolidated Berea sandstone with octane as the oil phase. After the calibration scans the core was flooded with water and this was followed by injection of oil. The core was then waterflooded till the oil saturation reached 30\%. Air was then injected slowly at the rate of $0.16 \mathrm{cc} / \mathrm{min}$ from the top and fluids were allowed to drain from the bottom. Air injection was used in this experiment to speed up the drainage process. However, the injection rate was chosen to be sufficiently low that the pressure gradient in the air phase was small and the analysis to find $k_{r o}$ and $k_{r w}$ was still valid. The average oil saturation in the top half of the core after 1600 hours of drainage was $18.9 \%$.

From these experiments we obtained the saturations as a function of time and distance along the length of the core. The capillary pressures were obtained from analog experiments and were used along with the saturation measurements in Eqs. 10.16 and 10.17 to obtain three phase relative permeabilities.

\subsection{Results and Discussion}

Fig. 10.8 shows the water relative permeabilities for experiments performed on sand with octane as the oil phase. Water relative permeabilities were not obtained for experiments 4 and 5 since the initial water saturations here were low and there was very little displacement of water. For experiment 6 , only data from the top half of the core was used to obtain the relative permeability. The initial water saturation in the lower half of the core was not uniform and this lead to a large scatter in the computed $k_{r w}$.

Fig. 10.9 gives the oil relative permeabilities for all six experiments.

A study of Figs. 10.8 and 10.9 reveal the following features:

1. To within experimental error $k_{r w}$ is independent of initial condition. Other work[155] on three phase flow has also shown that $k_{r w}$ is a function only of its own saturation, and is independent of $S_{o i}$. $k_{r w}$ falls very steeply with $S_{w}$, and $k_{r w} \sim S_{w}{ }^{a}$, with $a \approx 5$. To within experimental error, $k_{r w}$ is the same for the two phase and three phase experiments performed on sand.

2. $k_{r o}$ is independent of $S_{o i}$. (Compare $k_{r o}$ for experiments 1,2 and 3).

3. At high oil saturations, $S_{o}>S_{o r}, k_{r o}$ is independent of spreading coefficient. $k_{r o} \sim S_{o}{ }^{a}$, with $a \approx 4$. (Compare $k_{\text {ro }}$ for experiments 1,4 and 5 ).

4. At low oil saturations, $S_{o}<S_{o r}, k_{r o}$ is sensitive to spreading coefficient. $k_{r_{o}}$ is slightly higher for hexane than octane, while for decane, $S_{o}$ never falls below 0.09. (Compare $k_{r o}$ for experiments 1, 4 and 5).

5. For $S_{o}<S_{o r}$, with the exception of decane, we see $k_{r o} \sim S_{o}^{a}$, with $a \approx 2$.

Observation 5 is perhaps the most remarkable and suggests a very simple universal form for $k_{r o}$ at low saturation for water-wet media. In confirmation of this hypothesis, Fig. 10.10 shows the oil relative permeability in a Clashach sandstone.[147] Fig. 10.11 shows the $k_{r o}$ for three phase flow 

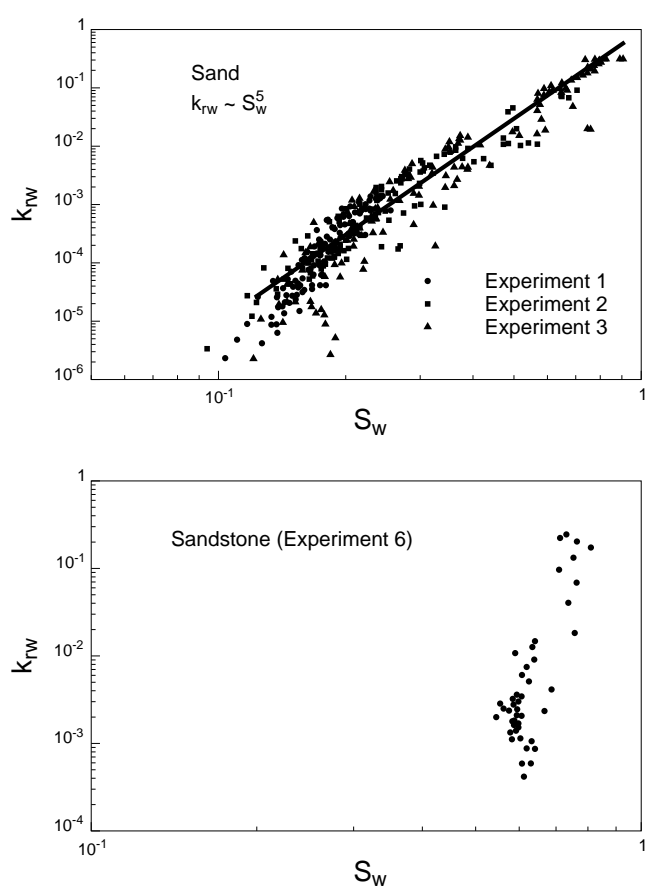

Figure 10.8: Water relative permeability as a function of water saturation. The straight line in the top figure is a best fit through the data. Notice that the results of the three experiments lie on a single curve, which is similar to the two phase results in Fig. 10.5.

in bead packs with analog fluids.[76] In both cases we see $k_{r_{o}} \sim S_{o}^{2}$ at low oil saturation. Similar results were obtained by Goodyear and Jones.[75]

The quadratic form for $k_{r o}$ is a direct consequence of layer drainage at the pore scale.[68] Fig. 10.12 shows an idealized corner in a pore space. The essential features are that the rock is waterwet and that there are roughness, grooves or crevices that act as conduits for wetting fluid, even when the center of the pore is filled with gas. Oil may reside in a layer of order of microns in thickness, wedged between the water and gas, as shown. At low $S_{o}$, gas occupies most of the pore space, while oil is primarily confined to layers. The oil saturation is proportional to the crosssectional area of the layer. Simple analysis can show that the layer conductance and hence relative permeability is proportional to oil area squared. Hence, macroscopically, $k_{r o} \sim S_{o}^{2}$. At higher $S_{o}$, $k_{r o}$ is governed by the arrangement of pores filled with oil and layer drainage no longer dominates the flow. In this regime, pore network modeling can successfully predict the $k_{r o} \sim S_{o}^{4}$ seen in these experiments. Since it is layer drainage that accounts for the displacement of waterflood residual oil during inert gas injection, we postulate that the layer drainage regime is observed for $S_{o}<S_{o r}$. This is consistent with the experimental results.

Layer drainage was observed for both hexane and for octane, whose initial spreading coefficient was slightly negative. Both hexane and octane may be loosely considered spreading oils in the sense that their equilibrium spreading coefficients are probably close to zero and they readily form oil layers in the pore space. Note, however, that octane does not spread on a flat brine surface $\left(C_{s}<0\right)$, but does show the layer drainage regime with a very low final oil saturation.

While n-decane may form layers in the pore space in micromodel experiments, $[115,47]$ the range of capillary pressures for which these layers are stable decreases with decreasing spreading coefficient and network modeling has shown that the layer drainage regime may not be observed for a decane system.[68] Experiment 5 confirms this: there is an apparent $S_{\text {or }} \approx 0.1$ and no layer 
drainage regime. This implies that for n-decane there was no recovery through oil layers.

Notice that the $k_{r w}$ curves do not display a layer drainage regime $\left(k_{r w} \sim S_{w}^{2}\right)$, but that $k_{r w}$ falls very sharply with $S_{w}$. This is because even at low $S_{w}$, the water being the most wetting phase, completely fills some small pores and crevices which may be poorly connected. This makes $k_{r w}$ sensitive to the exact arrangement of water-filled regions of the pore space, similar to the $k_{r o}$ regime for $S_{o}>S_{o r}$.

\subsection{Summary and Conclusions}

1. Dual-energy scanning using a modified CT scanner was used to obtain independent measurements of two saturations as a function of time and distance along the length of the core. Capillary pressure was obtained from analog experiments.

2. In six gravity drainage experiments on water-wet porous media, hexane, octane and decane were used as the oil phases to study the effect of spreading coefficient on relative permeability.

3. Very low oil saturations are observed during three phase gravity drainage experiments for octane and hexane.

4. For the spreading systems (hexane and octane), the oil relative permeability, $k_{r_{o}} \sim S_{o}^{4}$ for $S_{o}>S_{o r}$ and then exhibited a crossover to $k_{r o} \sim S_{o}^{2}$ for lower oil saturations.

5. This quadratic form of the relative permeability was seen down to oil saturations as low as $1.5 \%$. This is consistent with layer drainage at the pore scale.

6. In the non-spreading system (decane), this layer drainage regime was not observed

\subsection{Nomenclature}

$C_{s}=$ spreading coefficient

$k=$ absolute permeability

$k_{r p}=$ relative permeability to phase $p$

$p_{p} \quad=$ pressure of phase $p$

$p_{c g o}=$ gas oil capillary pressure

$p_{\text {cow }}=$ oil water capillary pressure

$S_{p}=$ saturation $(\mathrm{p}=\mathrm{o}, \mathrm{g}, \mathrm{w})$

$S_{\text {or }}=$ waterflood residual oil saturation

$S_{o i}=$ initial oil saturation

$u_{p}=$ flow velocity of phase $p$

\section{Greek Letters}

$\gamma=$ interfacial tension

$\nu=\mathrm{x}$-ray attenuation of material

$\phi \quad=$ porosity

$\rho_{p}=$ density of phase $p$

\section{Subscripts}

$1=$ first energy level

$2=$ second energy level

$i=$ initial condition

$g=$ gas

$o \quad=$ oil

$p=$ phase

$r \quad=$ rock

$w \quad=\quad$ water (brine) 

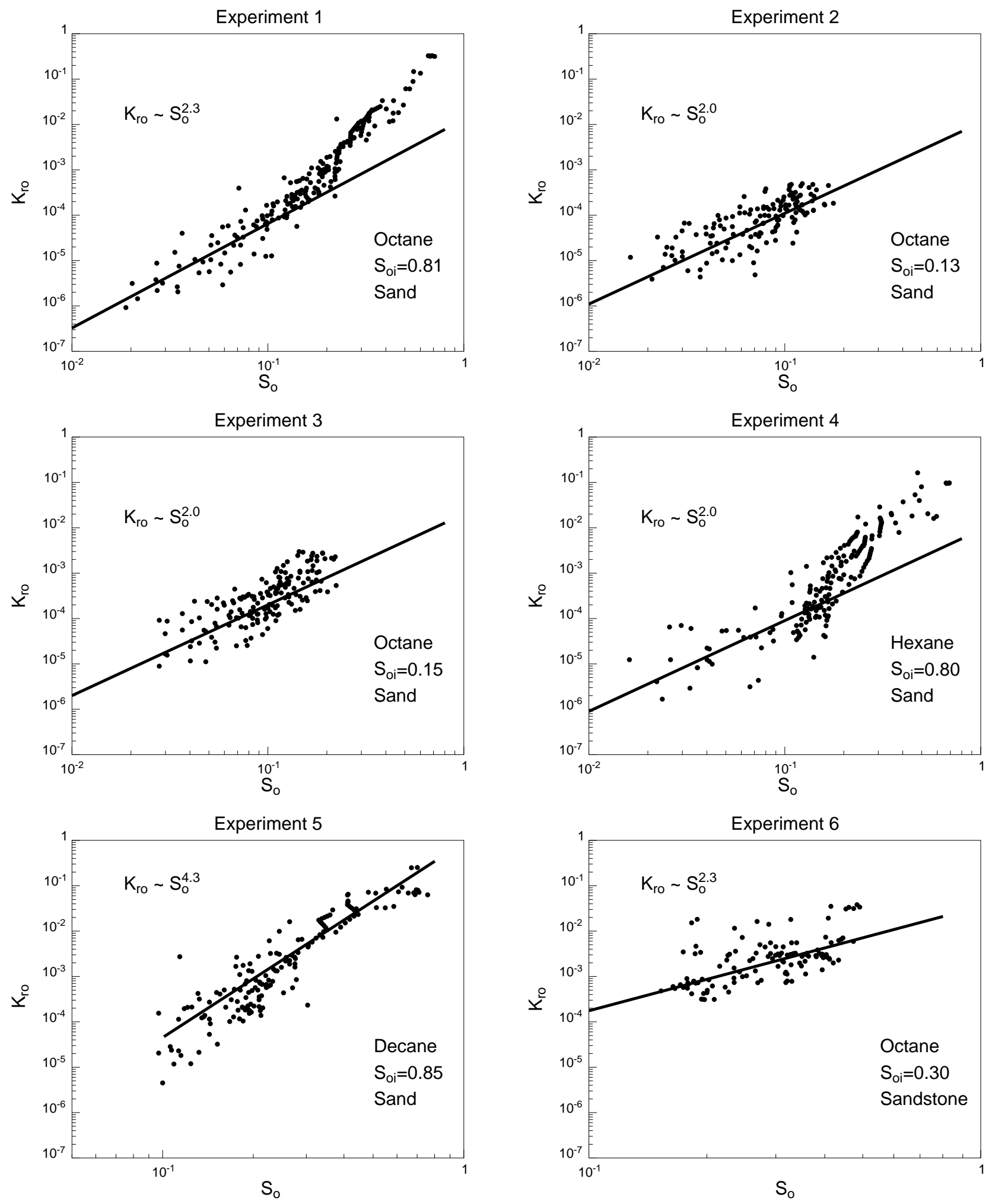

Figure 10.9: Measured oil relative permeabilities. The straight lines are best fits to the data at low oil saturation. Notice that all the curves show an approximately quadratic form for the oil relative permeability at low oil saturations, except for the non-spreading decane system. 


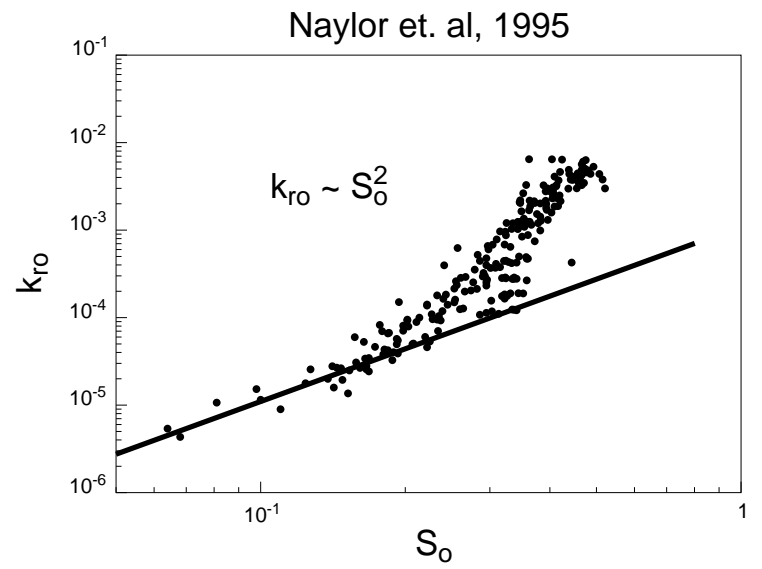

Figure 10.10: Oil relative permeability from Naylor et al.. [147]

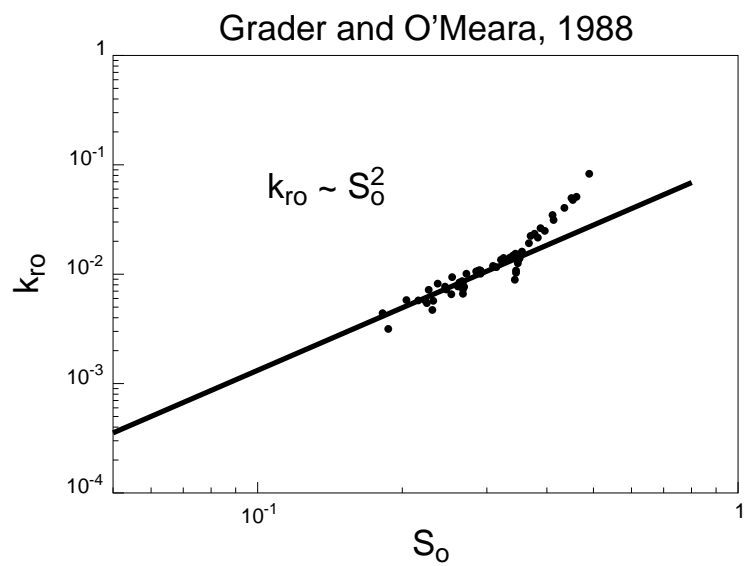

Figure 10.11: Oil relative permeability from Grader and O’Meara. [76]

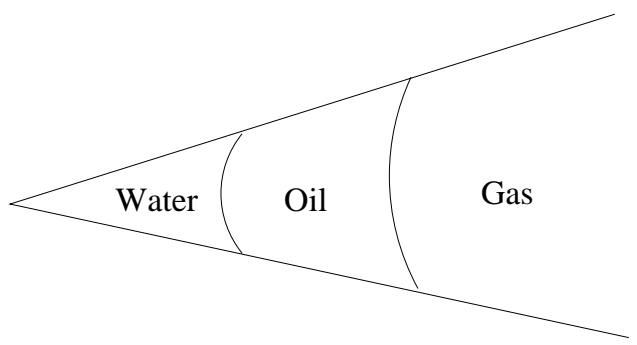

Figure 10.12: Fluid distribution in the corner of a pore space. 


\subsection{Appendix}

\subsubsection{Three Phase Saturation Calculation}

The equations for three phase saturation calculations using dual-energy CT scanning are derived in the following section. For each energy level, the CT numbers of a core containing three fluids at energy levels 1 and 2 are given by the equations:

$$
\begin{aligned}
& C T_{1}=(1-\phi) \nu_{r 1}+\phi s_{g} \nu_{g 1}+\phi s_{o} \nu_{o 1}+\phi s_{w} \nu_{w 1} \\
& C T_{2}=(1-\phi) \nu_{r 2}+\phi s_{g} \nu_{g 2}+\phi s_{o} \nu_{o 2}+\phi s_{w} \nu_{w 2}
\end{aligned}
$$

where $\nu$ is the xray attenuation coefficient of the material.

Also

$$
S_{g}=1-S_{o}-S_{w}
$$

This gives us three equations with three unknowns.

The CT numbers for the dry scans are

$$
C T_{g r 1}=(1-\phi) \nu_{r 1}+\phi \nu_{g 1}
$$

and

$$
C T_{g r 2}=(1-\phi) \nu_{r 2}+\phi \nu_{g 2}
$$

This gives

$$
\begin{gathered}
S_{o}=\frac{\left[C T_{1}-C T_{g r 1}\right]\left(\phi \nu_{w 2}-\phi \nu_{g 2}\right)-\left[C T_{2}-C T_{g r 2}\right]\left(\phi \nu_{w 1}-\phi \nu_{g 1}\right)}{\left(\phi \nu_{o 1}-\phi \nu_{g 1}\right)\left(\phi \nu_{w 2}-\phi \nu_{g 2}\right)-\left(\phi \nu_{o 2}-\phi \nu_{g 2}\right)\left(\phi \nu_{w 1}-\phi \nu_{g 1}\right)} \\
S_{w}=\frac{\left[C T_{2}-C T_{g r 2}\right]\left(\phi \nu_{o 1}-\phi \nu_{g 1}\right)-\left[C T_{1}-C T_{g r 1}\right]\left(\phi \nu_{o 2}-\phi \nu_{g 2}\right)}{\left(\phi \nu_{o 1}-\phi \nu_{g 1}\right)\left(\phi \nu_{w 2}-\phi \nu_{g 2}\right)-\left(\phi \nu_{o 2}-\phi \nu_{g 2}\right)\left(\phi \nu_{w 1}-\phi \nu_{g 1}\right)}
\end{gathered}
$$

To account for the influence of the rock, add and subtract $(1-\phi)\left(\nu_{r i}\right)$ for $\mathrm{i}=1$ and 2 into each parentheses.

The equations now become

$$
\begin{aligned}
S_{o} & =\frac{\left[C T_{1}-C T_{g r 1}\right]\left(C T_{w r 2}-C T_{g r 2}\right)\left[C T_{2}-C T_{g r 2}\right]\left(C T_{w r 1}-C T_{g r 1}\right)}{\left(C T_{o r 1}-C T_{g r 1}\right)\left(C T_{w r 2}-C T_{g r 2}\right)-\left(C T_{o r 2}-C T_{g r 2}\right)\left(C T_{w r 1}-C T_{g r 1}\right)} \\
S_{w} & =\frac{\left[C T_{2}-C T_{g r 2}\right]\left(C T_{o r 1}-C T_{g r 1}\right)\left[C T_{1}-C T_{g r 1}\right]\left(C T_{o r 2}-C T_{g r 2}\right)}{\left(C T_{o r 1}-C T_{g r 1}\right)\left(C T_{w r 2}-C T_{g r 2}\right)-\left(C T_{o r 2}-C T_{g r 2}\right)\left(C T_{w r 1}-C T_{g r 1}\right)}
\end{aligned}
$$




\subsubsection{Calculating Porosity}

The porosity of the core can be obtained by scanning the core dry and then scanning it fully saturated with water(brine).

$$
\phi=\frac{C T_{w r}-C T_{g r}}{C T_{w}-C T_{g}}
$$

where $C T_{w}$ and $C T_{g}$ are the pure fluid $\mathrm{CT}$ numbers for water and gas respectively. They are obtained by scanning the pure fluids inside the coreholder in place of the core. This helps in eliminating beam hardening effects due to the coreholder material and artifacts that effect the fluid CT number. $C T_{w r}$ and $C T_{g r}$ are the CT numbers of the core fully saturated with water and air respectively.

\subsubsection{Error Analysis}

All CT measurements have errors associated with them. Error is defined as the standard deviation in CT number on a pixel by pixel basis of a 2" diameter fused quartz sample in the same setup as the experiments. A fused quartz sample was placed in the coreholder and scanned at the two energy levels. The error in CT number for energy level 1 is 2.6 and for energy level 2 is 5.6.

From this we find that the error in the oil and water saturations calculated from Eqs. 10.31 and 10.32 is $4.3 \%$ and $9.3 \%$ respectively. The error in the gas saturation is $13.6 \%$. In our calculations for relative permeability, we average the saturation over a slice (approximately 27500 pixels). If we assume the error to be independent and random, then the average error in the oil saturation over the slice is $0.026 \%$ The average errors in the water and gas saturations are $0.056 \%$ and $0.082 \%$ respectively. These errors are tiny and result in very little scatter in computed relative permeability. Thus the observed scatter in the measurements is attributed to the local inhomogeneities in the permeability and porosity of the porous media, together with non-uniform initial conditions. Smoothing the saturation profiles would give us less scatter in the relative permeability curves. 


\title{
11. Three-Phase Relative Permeability of Water-Wet, Oil-Wet, and Mixed-Wet Sandpacks
}

\author{
David A. DiCarlo, Akshay Sahni, and Martin J. Blunt
}

In this chapter we present experimental studies of three-phase flow in water-wet, oil-wet, mixedwet, and fractionally-wet sandpacks. In oil reservoirs, three-phase flow will occur during immiscible gas injection, gas cap expansion, and thermal flooding among other processes. All of these processes are affected by the wettability of the reservoir. We use sandpacks as our model porous medium, and affect the wettability of the sand by aging it in crude oil. We make the mixed-wet pack by invading a water-filled water-wet pack with crude oil and aging for a week. This process mimics wettability changes in reservoir settings, leading to a realistic arrangement of wettability at the pore scale. We characterize the wettability of each sand pack by measuring the capillary pressure curves. We obtain the oil and water relative permeabilites during three-phase gravity drainage, by measuring the saturation in-situ using CT scanning. In another experiment, we measure pressure gradients in the gas phase to obtain the gas relative permeability. Thus we determine all three relative permeabilities as a function of saturation for each wettability. We find that under uniform wetting, the relative permeabilities of the most-wetting phase (water in a water-wet pack, oil in an oil-wet pack) are similar. However, the relative permeabilities of the intermediate-wet phase (oil in a water-wet pack, water in a oil-wet pack) are very different at low saturations, with spreading oils showing a characteristic layer drainage regime. The mixed-wet pack also shows the layer drainage regime. We also find that the gas relative permeability is smaller in an oil-wet medium than in a water-wet medium. We explain the observations in terms of wetting, spreading, and the pore scale configurations of fluid.

\section{$11.1 \quad$ Introduction}

A knowledge of three-phase (water, oil, and gas) flow in porous media is essential for predicting enhanced oil recovery and the migration of nonaqueous phase pollutants. In two-phase flow, one phase will wet the porous medium more than the other phase. This wetting phase occupies the smaller pores, crevices, and corners while the nonwetting phase occupies the larger pores with the exact arrangement determined by the capillary pressure. Regardless of which pair of fluids is used (gas/water, oil/water, gas/oil) the positioning of the fluids are likely to be similar at the same saturations. In contrast, for three-phase flow there will be an intermediate wetting phase which will be positioned uniquely in the porous medium, affecting macroscopic properties such as relative permeability and residual saturation.

We measure three-phase relative permeabilities during the gravity driven displacement of oil and water by gas, which is called gravity drainage. This is an important three-phase process that occurs during gas cap expansion in an oil reservoir and when nonaqueous phase pollutants migrate through an unsaturated soil. Gravity drainage is also relatively easy to study experimentally and its analysis avoids consideration of hysteresis effects, which can be very significant in three-phase flow [193, 68].

Several authors[49, 114, 227] have shown that low oil saturations can be reached during threephase displacements. Most experiments have been performed on uniformly water-wet media (see

\footnotetext{
${ }^{1}$ The material in this chapter was published as "Three-Phase Relative Permeability of Water-Wet, Oil-Wet, and Mixed-Wet Sandpacks," SPE Journal (2000) 5, 82-91.
} 
Baker[6] for a review). Oak et al. [155] studied three-phase relative permeabilities in an intermediatewet Berea sandstone. Vizika and Lombard[217] studied three-phase drainage for water-wet, oil-wet, and fractionally-wet systems. Jerauld[103] developed a model for three-phase relative permeability based on two-phase measurements for Prudhoe Bay, which is a mixed-wet reservoir.

Sahni et al.[186] used CT scanning to measure oil and water relative permeability in water-wet media and studied the effect of spreading coefficient. Zhou and Blunt[228] performed three-phase gravity drainage in fractionally-wet sandpacks. They measured the saturation distribution at the end of drainage and interpreted the results in terms of the pore scale arrangement of fluid. DiCarlo et al.[42] measured three-phase relative permeabilities for oil-wet and fractionally-wet packs.

The fractionally-wet media studied in the literature were composed of mixtures of oil-wet and water-wet grains. While this is a convenient way of varying the wettability, it does not necessarily represent the real distribution of wettability in a natural setting. An alternative approach is to mimic a physical sequence of wettability changes in the laboratory, leading to a medium whose pore-scale pattern of wettability represents reservoir rocks. This is achieved by flooding a waterwet water-filled pack with a crude oil and then aging for several days. This induces a wettability change on the sand surfaces contacted by oil (see, for example Buckley et al. [24]). Smaller pores that are water-filled and the corners of the pore space will remain water-wet. We will call such systems mixed-wet.

In this chapter we extend the work of Sahni et al.[186] and DiCarlo et al.[42] to mixed-wet media. The description of the experiments follows DiCarlo et al.[42], and for the sake of completeness, we present all our results in water-wet, oil-wet, fractionally-wet, and mixed-wet media. We characterize the wettability by measuring two-phase (water/oil) capillary pressure curves. We measure the gas relative permeability using an analogue experiment in which we directly measure the gas saturation and pressure gradient. Thus we obtain all three permeabilities for three-phase gravity drainage, in water-wet, oil-wet, mixed-wet and fractionally-wet sandpacks. We explain the results in terms of the pore scale fluid arrangements.

\subsection{Materials and Methods}

We chose sandpacks as our porous media as they are easy to characterize and they are easily sectionable for destructive saturation measurements. We used clean industrial sand (no. 60, Corona Industrial Sand Co., Corona, CA) which was initially water-wet. The sand was sieved with a size 120 sieve to remove any fine particles. We made $15 \mathrm{~kg}$ of the sand oil-wet by soaking initially dry sand in a mixture of $20 \%$ crude oil (Thums Inc., Long Beach, CA) and $80 \%$ iso-octane for 24 hours [25]. This oil-wet sand was then rinsed with iso-octane and air-dried. The fractionally-wet sand was a 50-50 mixture of the oil and water-wet sands.

Mixed-wet sandpacks were created from water-wet packs, by first saturating the pack with a $0.01 \mathrm{M} \mathrm{NaBr}$ brine ( $\mathrm{pH} 4$ ). We then displaced the brine with 5 pore volumes (PV) of the crude/isooctane mixture, yielding a pack with a water saturation of $S_{w} \approx 0.2$. The column was then left to age for a week. After the aging the crude mixture was displaced with iso-octane until the effluent ran clear. Finally, the pack was flooded by n-octane, replacing the iso-octane, then flooded with the working water phase (either distilled water or $10 \% \mathrm{NaBr}$ brine, see below) to replace the brine.

For our fluids, we chose n-hexane, n-octane, or n-decane for the oil phase, distilled water or $10 \% \mathrm{NaBr}$ by weight brine for the water phase, and air for the gas phase. The density of the brine was measured volumetrically, and the density of the oils was taken from the CRC Handbook [37]. Viscosities were measured using a viscometer. Interfacial tensions were measured using the pendant drop technique [1]. Table 11.1 shows the fluid properties. All experiments were performed at room temperature and pressure.

In all of the experiments, the sandpacks were set to connate water saturation $\left(S_{w c}\right)$ or residual 
Table 11.1: Fluid densities $\left[\rho_{w}=1069 \mathrm{~kg} / \mathrm{m}^{3}\right]$, viscosities $\left[\mu_{w}=1.23 \mathrm{cP}\right]$, and interfacial tensions $\left[\gamma_{g w}=72.0 \mathrm{mN} / \mathrm{m}\right]$.

\begin{tabular}{ccccc}
\hline Oil & $\begin{array}{c}\rho_{o} \\
\left(\mathrm{~kg} / \mathrm{m}^{3}\right)\end{array}$ & $\begin{array}{c}\mu_{o} \\
(\mathrm{cP})\end{array}$ & $\begin{array}{c}\gamma_{\text {ow }} \\
(\mathrm{mN} / \mathrm{m})\end{array}$ & $\begin{array}{c}\gamma_{\text {go }} \\
(\mathrm{mN} / \mathrm{m})\end{array}$ \\
\hline n-hexane & 659 & 0.30 & 50.5 & 18.0 \\
\hline n-octane & 703 & 0.51 & 51.2 & 21.1 \\
\hline n-decane & 730 & 0.84 & 51.4 & 23.7 \\
\hline
\end{tabular}

oil saturation $\left(S_{o r}\right)$ by the following procedure. For uniform-wet packs (water-wet and oil-wet), the core was saturated with the most wetting fluid (water for water-wet, oil for oil-wet) and then displaced with $5 \mathrm{PV}$ of the intermediate wetting fluid (oil or water). This produced an initial condition of $S_{w c}$ for the water-wet pack and $S_{\text {or }}$ for the oil-wet pack. Initial conditions of $S_{\text {or }}$ for the water-wet pack and $S_{w c}$ for the oil-wet pack were achieved by an additional $5 \mathrm{PV}$ flooding of the most-wetting fluid. For the fractionally-wet pack there is no most-wetting fluid, so an initial condition of $S_{o r}$ was achieved by flooding a $100 \%$ oil-saturated pack with water, and $S_{w c}$ was achieved by flooding a $100 \%$ water-saturated pack with oil. For the mixed-wet pack, since its last flood was by the water phase, it was already at $S_{o r}$. An additional flood of oil was used to achieve $S_{w c}$. For each experiment, a new mixed-wet pack was created from water-wet sand except for the three-phase drainage from $S_{w c}$. In this case, due to a need to recalibrate the CT scanner, the core that was used for the drainage from $S_{\text {or }}$ was dried out completely by flowing dry air through the column for 4 weeks. The column was then filled with oil. This was followed by 2 PV of isopropanol to miscibly displace the oil, and then $5 \mathrm{PV}$ of brine. Finally, $5 \mathrm{PV}$ of octane was injected to produce the initial condition of $S_{w c}$. All displacements were performed in a gravitational stable configuration.

\subsubsection{Capillary Pressure Curves}

To characterize the wettability changes, we measured two-phase water/oil capillary pressure curves using a gravitational equilibrium technique. Distilled water was used for the water phase, and n-octane for the oil phase. The desired medium was packed through a continuous pour into a $72 \mathrm{~cm}$ column ( $2.54 \mathrm{~cm}$ inner diameter) and sealed at both ends with rubber stoppers. The column consisted of 24 separate $3 \mathrm{~cm}$ long polycarbonate sections held together with teflon shrink tubing, which made it easier for accurate sectioning of the column for saturation measurements. For water injection (which we will call imbibition even for nonwater-wet media), the medium was initially set to $S_{w c}$ (see above). The imbibition began by connecting the bottom of the column to a large tank of water (whose height and thus the water head did not change during the imbibition) and the top to a smaller volumetric tank of oil (whose height changed slightly during the imbibition). Using calculations described below, the oil and water heads were chosen such that the water would imbibe throughout the porous medium. An identical technique was used for drainage curves (oil invasion), except that the column was initially set to $S_{o r}$, and the heads set for drainage. Thus the curves obtained are for secondary imbibition and secondary drainage.

Once the tanks were attached, the columns were left for roughly 1 week to reach gravitational equilibrium. Then the columns were sectioned, their contents were dropped into a flask containing $25 \mathrm{ml}$ of isopropanol, which created a single-phase mixture suitable for analysis, and the oil and water saturations were measured using a gas chromatograph (GC). From this we obtained the 
saturation profile versus vertical position.

Assuming that the column is at capillary equilibrium, the profile can be described in terms of the capillary pressure. We define $z$ positive downward and $z=0$ at the top of the column. Let $z_{o}$ be the final height of the oil level in the oil tank, and $z_{w}$ the final height of the water in the water tank. Since the column is at equilibrium, the pressure of each phase $i$ as a function of $z$ is given by

$$
P_{i}=\rho_{i} g\left(z-z_{i}\right),
$$

where $\rho$ is the density, and $g$ the gravitational constant. Thus the capillary pressure is

$$
P_{c}=P_{o}-P_{w}=\left(\rho_{o}-\rho_{w}\right) g z-\left(\rho_{o} g z_{o}-\rho_{w} g z_{w}\right) .
$$

The water and oil heads were chosen such that the capillary fringe would be roughly in the center of the column after imbibition. If this did not occur, the experiment was repeated with different heads.

\subsubsection{Oil and Water Relative Permeabilities}

Before and during gravity drainage, we used a dual energy CT scanner to obtain in-situ measurements of the water and oil saturations[186]. To achieve good CT contrast, the $10 \% \mathrm{NaBr}$ brine was used for the water phase, and either n-hexane, n-octane, or n-decane was used for the oil phase. The core holders were standard Hassler-type with an overburden fluid (water at 50 psi) and a rubber sleeve. The sand was packed into the rubber sleeve of diameter $7.6 \mathrm{~cm}$ and length 67 $\mathrm{cm}$ (87 $\mathrm{cm}$ sleeves were used for water-wet sand). The core was mounted on a vertical positioning system in a Picker 1200X CT machine, which scanned in the horizontal plane. Scans of width 5 $\mathrm{mm}$ were taken every $2 \mathrm{~cm}$ along the core and at energy levels of $80 \mathrm{kV}$ and $140 \mathrm{kV}$.

The experiment was calibrated by scanning the core when it was dry, when it was saturated with brine, and when it was saturated with oil. Using this calibration and a standard linear interpolation of CT number (i.e. attenuation coefficient), the water, oil, and air saturations could be obtained during a three-phase experiment. The saturations were observed to be uniform over each slice, except within $2 \mathrm{~cm}$ of the capillary fringe where the scans showed regions of high and low saturations. In either case, the saturations were averaged over each slice to give the average water, oil, and air saturation every $2 \mathrm{~cm}$.

We can estimate the accuracy of the saturation by measuring the standard deviation on a pixel basis, and then assuming the standard deviation of the average saturation is the standard deviation of the mean. This gives a typical accuracy of $\Delta S_{i} \approx 0.0002$. This is likely to be too small due to nonlinear systematic variations such as beam hardening. In practice, when the core is at its initial condition with water and oil, but no gas, we measure a typical gas saturation value of $S_{g} \approx \pm 0.01$. Thus we estimate our accuracy for each phase to be $\Delta S_{i} \approx 0.01$.

Before drainage each core was set to connate water $S_{w c}$ or residual oil $S_{\text {or }}$ saturation as described before. Once the desired initial condition was reached, gas (air) was allowed to enter the top of the system, while water and oil drained out of the bottom under gravity. Before entering the column the air passed through a bubbler filled with water and a bubbler filled with oil to saturate the air with water and oil vapor. The bubblers also provided a one-way pathway for the air such that no oil and water vapor could leave the column through the top port. The height of oil and water that the entering air bubbled through was less than $1 \mathrm{~cm}$, so the pressure boundary conditions were $P_{g}=0 \mathrm{~Pa}$ at the outlet and $P_{g} \approx-100 \mathrm{~Pa}$ at the inlet.

The pack was periodically scanned over a period of several weeks to record the saturation distribution versus time. Figure 11.1 shows the saturation profile of octane and water at various times for a water-wet column that was initially at $S_{w c}$. 


\section{Water-Wet Gravity Drainage}
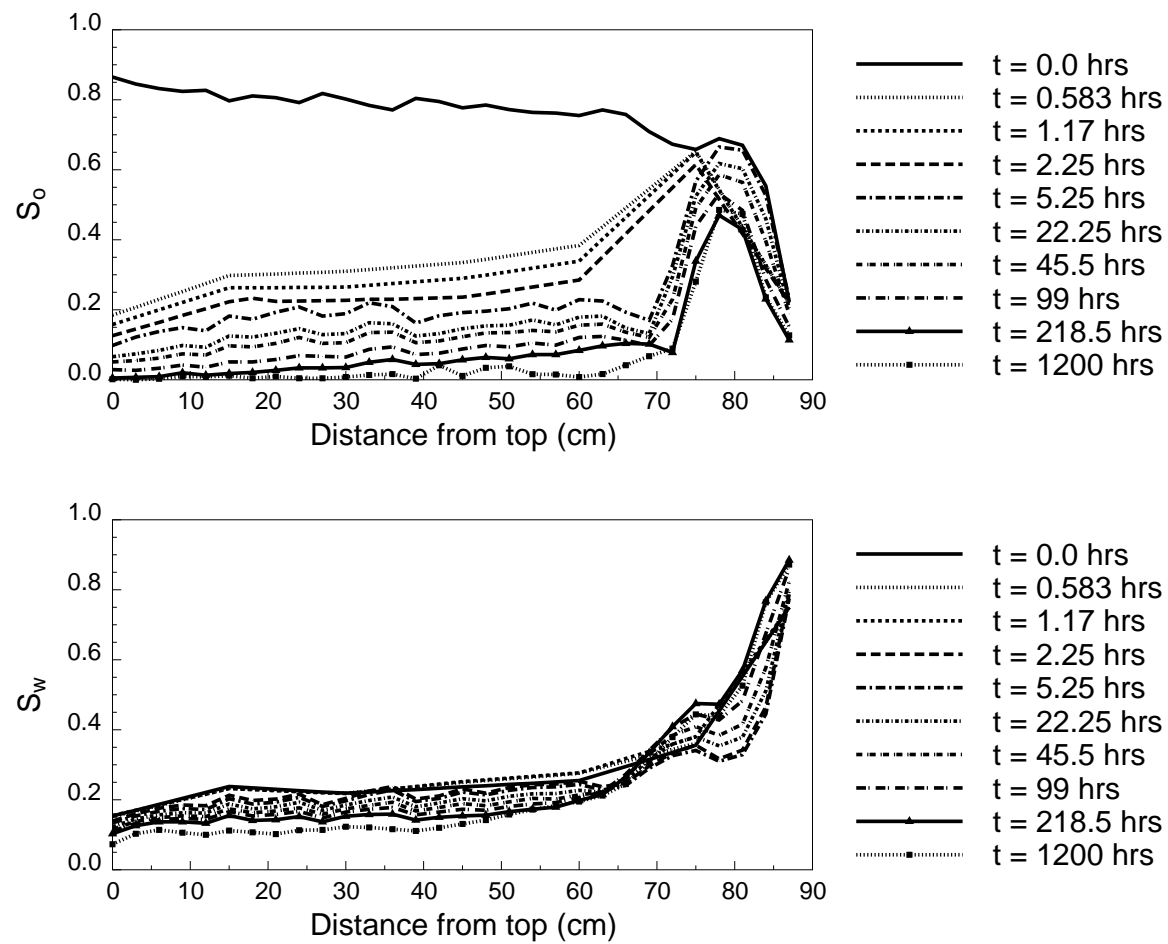

Figure 11.1: Oil (octane) and water saturation profiles versus time during gravity drainage. The sand was water-wet and the initial condition was connate water saturation $S_{w c}$. Oil and water permeabilities can be calculated directly from the measured distributions. 
We can compute the oil and water relative permeabilities at each measured position along the column and for each time from the known time evolution of the saturation profile $S_{i}(z, t)$. We begin with Darcy's law for each phase in a 1-dimensional vertical displacement,

$$
u_{i}=-\frac{k k_{r i}}{\mu_{i}}\left(\frac{\partial P_{i}}{\partial z}-\rho_{i} g\right),
$$

where $u$ is the flux, $k$ is the permeability (20 Darcy for our sand), $k_{r}$ is the relative permeability, $\mu$ is the viscosity, and $z$ is the vertical distance taken to be positive downward. We calculate the flux past position $z$ for time interval $t_{1}, t_{2}$ by integrating the change in the saturation of each phase between scans at times $t_{1}$ and $t_{2}$,

$$
u_{i}\left(z, t_{1}, t_{2}\right)=\frac{\phi}{\left(t_{2}-t_{1}\right)} \int_{0}^{z} S_{i}\left(z^{\prime}, t_{1}\right)-S_{i}\left(z^{\prime}, t_{2}\right) d z^{\prime},
$$

where $\phi$ is the porosity.

The only remaining unknown is the pressure gradient $\left(\partial P_{i} / \partial z\right)$. This can be found directly using tensiometers, or indirectly by calculating the pressures from the saturations and a known capillary pressure curve[186]. In practice the indirect method has difficulties for column positions near and below the capillary fringe as the quantity $\partial S_{i} / \partial z$ is large, and the capillary pressure gradient is very close to the gravitational gradient. Thus any small errors in the saturation measurement correspond to large errors in the measured permeability.

Fortunately, we notice that near the top of the column the saturation is roughly constant with position $\left(\partial S_{i} / \partial z \approx 0\right)$. Thus we assume that $\partial P_{i} / \partial z<<\rho_{i} g$ and ignore the capillary pressure gradient for positions over a region were the saturation data satisfies the above condition (between 20 and $50 \mathrm{~cm}$ in Fig. 11.1). For the shorter oil-wet, mixed-wet, and fractionally-wet cores, the above condition was satisfied only between 10 and $30 \mathrm{~cm}$ from the top of the chamber. In essence, the CT scanner allows us to avoid capillary end effects in the determination of relative permeability, as we can choose a section over which the capillary pressure gradients are negligible compared to gravity.

For this region the relative permeability as a function of $z$ and time interval $t_{1}, t_{2}$ can be found by combining Eqs. 11.3 and 11.4 and dropping the pressure gradient,

$$
k_{r i}\left(z, t_{1}, t_{2}\right)=\frac{\mu_{i} \phi}{k \rho_{i} g\left(t_{2}-t_{1}\right)} \int_{0}^{z} S_{i}\left(z^{\prime}, t_{1}\right)-S_{i}\left(z^{\prime}, t_{2}\right) d z^{\prime} .
$$

Calculating the saturation as a function of $z$ and time interval $t_{1}, t_{2}$ as the average of the $t_{1}$ and $t_{2}$ saturations,

$$
S_{i}\left(z, t_{1}, t_{2}\right)=\left(S_{i}\left(z, t_{1}\right)+S_{i}\left(z, t_{2}\right)\right) / 2,
$$

we can then plot $k_{r i}$ as a function of $S_{i}$.

The accuracy of the technique is a function of the accuracy of the saturations, fluxes, and pressure gradients. The saturation accuracy is estimated previously to be \pm 0.01 . The flux accuracy is also likely to be good, as we know the time accurately and by integrating the saturations, the deviations are minimized further. The largest uncertainty is in the estimate of the pressure gradient. Simple two-phase simulations suggest that the assumption that the pressure gradient is small compared to the gravitational gradient is very good for short time scales $(<2$ weeks), but can be in error by up to $30 \%$ at long time scales ( $>6$ weeks).

\subsubsection{Gas Relative Permeability}

We have developed a procedure to measure the gas relative permeability in the sand packs. Distilled water was used for the water phase, and n-octane for the oil phase. The sand is packed 
Table 11.2: Amott indices, and residual saturations from the two-phase (water/oil) pressure saturation curves.

\begin{tabular}{ccccc}
\hline Wettability & $I_{w}$ & $I_{o}$ & $S_{o r}$ & $S_{w c}$ \\
\hline water-wet & 1 & 0 & 0.20 & 0.15 \\
\hline oil-wet & 0 & 0.05 & 0.05 & 0.20 \\
\hline mixed-wet & 0.10 & 0 & 0.10 & 0.10 \\
\hline fractionally-wet & 1.0 & 0 & 0.25 & 0.20 \\
\hline
\end{tabular}

through a continuous pour into a $60 \mathrm{~cm}$ column made out of 20 separate $3 \mathrm{~cm}$ long sections similar to that used in the capillary pressure curve measurements. Additionally, nine of the plastic sections in the column contain a small port through which the pressure of the gas phase can be measured. Thus we can measure eight separate pressure drops along the column during gas flow.

We first measure the single phase gas permeability by flowing known rates of $\mathrm{CO}_{2}$ (between 5 and $10 \mathrm{ml} / \mathrm{sec}$ ) through the column and measuring the pressure drops across each section using a water manometer. For two-phase measurements, the column is then filled with degassed water from below and the water is circulated until all of the original $\mathrm{CO}_{2}$ is displaced or dissolved. The column is then allowed to drain under gravity for about 1 hour, after which air is injected at a rate between 0.1 and $1 \mathrm{ml} / \mathrm{sec}$ at the top of the column, displacing additional water out the bottom. This injection is continued for several hours until the pressures have stabilized and the gas and water phases are in equilibrium. Due to capillary effects, the distribution of water varies along the sand pack, with a high water saturation (and low gas saturation) near the outlet and a low water saturation (and high gas saturation) near the inlet. The pressure drop is measured between each section, which in turn gives the gas relative permeability. The column is sectioned and the water and gas saturations are obtained gravimetrically. Low injection rates retained more water in the column and were used for low gas saturation measurements. There was no measurable effect on the gas relative permeabilities from the different gas injection rates.

For three-phase measurements a nearly identical procedure is used. The only differences are 1) the column is first filled to $S_{w c}$ and 2) the oil, water, and gas saturations are measured using the GC rather than gravimetrically. We did not measure the gas relative permeability for the mixed-wet sands.

\section{$11.3 \quad$ Results}

\subsubsection{Capillary Pressure Curves}

The first column of Figure 11.2 shows the drainage and imbibition curves for the sandpacks of different wettabilities. The curves are for two-phase water/oil displacements. From these curves, the calculated Amott wettability indices[3] and two-phase residual saturations are shown in Table 11.2.

For the oil-wet medium, the procedure we used to make the oil-wet pack should produce a uniform wettability on all the pore surfaces. The large hysteresis when compared to the water-wet case, the lack of spontaneous water imbibition, and the capillary pressures of the transition zones suggest that the water/oil contact angle is greater than $90^{\circ}$. Also, $S_{o r}$ is smaller than the water-wet case, implying that the oil remains connected at low saturations.

Measurements of contact angles in glass capillary tubes that had been treated by crude oil in the same manner as our sand found an oil/water contact angle of $152^{\circ}$.[70] Contact angles this large in 
Table 11.3: Drainage experiments from which three-phase oil and water relative permeabilities were obtained. The figure column shows where the data is displayed. The initial condition (I.C.) before drainage was either waterflood residual $\left(S_{o r}\right)$ or connate water $\left(S_{w c}\right)$.

\begin{tabular}{ccccc}
\hline Expt. & Figure $(\mathrm{s})$ & Oil & Porous Medium & I.C. \\
\hline 1 & $11.1,11.2,11.3,11.9$ & octane & water-wet sand & $S_{w c}$ \\
\hline 2 & $11.2,11.9$ & octane & water-wet sand & $S_{o r}$ \\
\hline 4 & 11.3 & hexane & water-wet sand & $S_{w c}$ \\
\hline 5 & $11.3,11.9$ & decane & water-wet sand & $S_{w c}$ \\
\hline 6 & 11.3 & octane & water-wet sandstone & $S_{o r}$ \\
\hline 7 & $11.2,11.9$ & octane & oil-wet sand & $S_{o r}$ \\
\hline 8 & 11.2 & octane & oil-wet sand & $S_{w c}$ \\
\hline 9 & 11.2 & octane & fractionally-wet sand & $S_{o r}$ \\
\hline 10 & 11.2 & octane & fractionally-wet sand & $S_{w c}$ \\
\hline 11 & $11.2,11.9$ & octane & mixed-wet sand & $S_{o r}$ \\
\hline 12 & $11.2,11.9$ & octane & mixed-wet sand & $S_{w c}$ \\
\hline
\end{tabular}

a porous medium should result in a significant degree of spontaneous oil imbibition.[48] However, the low oil Amott index of 0.05 shows that this is not the case. Overall, the oil-wet sandpack is only weakly oil-wet or almost neutrally-wet. While a direct measurement of oil/water contact angle on the sand grains is not possible, it is likely to be only slightly greater than $90^{\circ}$.

For the mixed-wet medium, we will assume that our procedure induced wettability changes as described by Salathiel [187] and Kovscek et al.[118]. When the crude oil contacts the solid, it can deposit surface active agents rendering these surfaces oil-wet. The larger pore spaces will tend to be oil-wet, as the smaller pores and throats remain water-filled. The corners and crevices of the large pore spaces will also remain water-filled and thus water-wet. Thus the oil-wet and water-wet portions will be discriminated by pore size.

The irreducible water saturation, $S_{w c}$ is slightly lower than in the water-wet sand, while the residual oil saturation, $S_{\text {or }}$, is between the water-wet and oil-wet cases. Also, the capillary pressures in the transition zone lie between the water-wet and oil-wet sands. The mixed-wet pack does not imbibe oil, while it imbibes some water. Overall its Amott indices imply a weakly water-wet or neutrally-wet system. It is possible that in our experiments the crude oil only renders the surfaces it contacts weakly oil-wet.

For the fractionally-wet media, since each sand grain is either water or oil-wet, each side of a pore will be either water or oil-wet. There will be no pore size discrimination for oil-wet and waterwet portions. Also the oil-wet and water-wet surfaces will be connected only randomly, producing pockets of oil-wet and water-wet regions. This description agrees with the observed large residual water $S_{w c}$ and large residual oil $S_{o r}$, as each fluid can get trapped in their isolated pockets [228]. But the observed capillary pressures in the transition zones are much closer to the water-wet sands, which is unexpected.

\subsubsection{Three-Phase Relative Permeability}

Table 11.3 lists all the three-phase drainage experiments and the figures which display the data. The experiments studied how the three-phase relative permeabilities varied as a function of the oil phase, the porous medium, and the initial condition of the pack. The second and third columns of 
Two-Phase Water/Oil $\mathrm{P}_{\mathrm{c}}-\mathrm{S}_{\mathrm{w}}$

a)
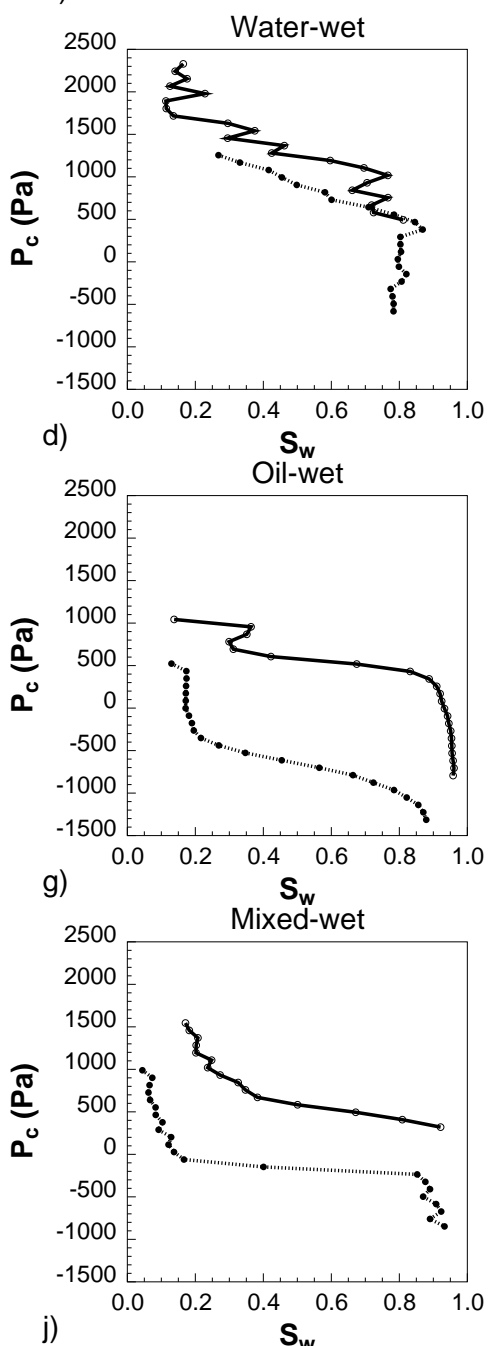$$
\text { j) }
$$

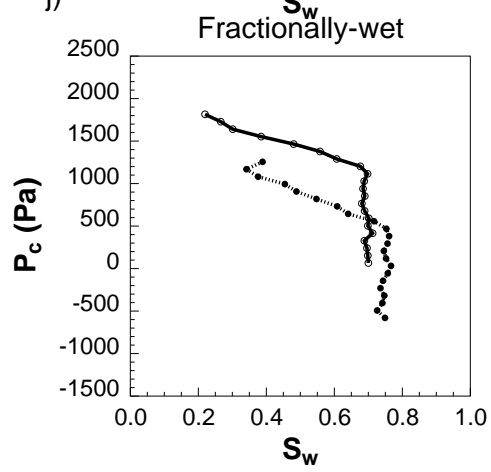

Three-Phase Oil k $\mathrm{ro}_{\mathrm{o}}$

b)
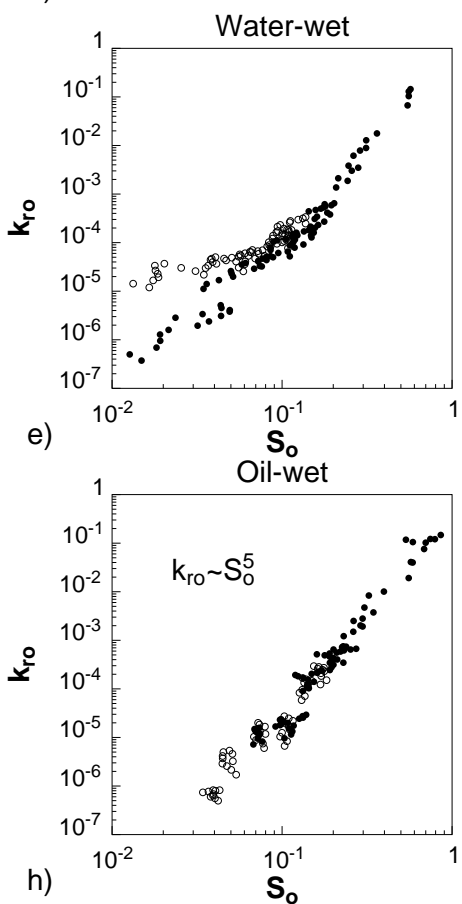

$1 \quad$ Mixed-wet

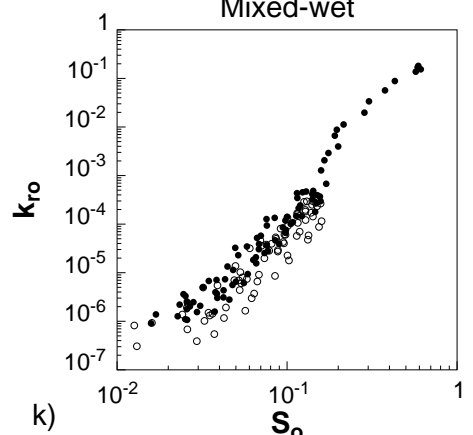

k)

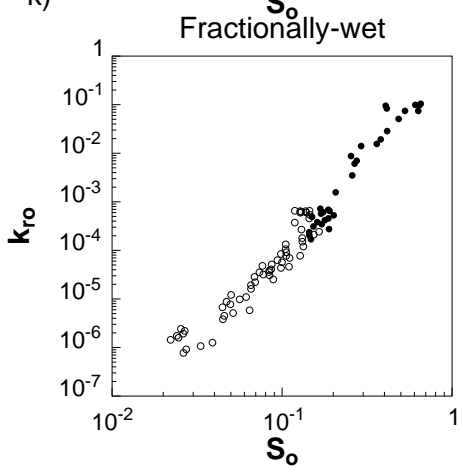

Three-Phase Water $\mathrm{k}_{\mathrm{rw}}-\mathrm{S}_{\mathrm{w}}$

c)
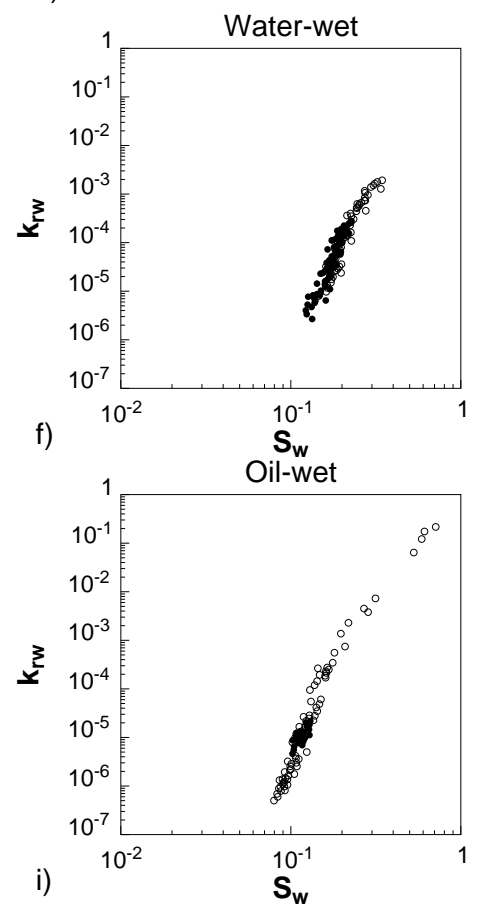

$1 \quad$ Mixed-wet
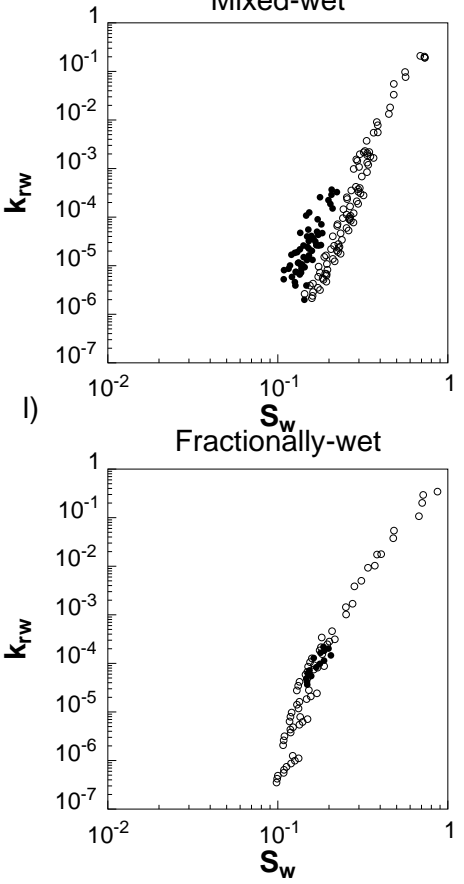

Figure 11.2: Measured two-phase (water/oil) capillary pressure curves and three-phase oil and water relative permeabilities with n-octane as the oil for water-wet, oil-wet, mixed-wet, and fractionallywet sand. Open symbols are drainages which started from residual oil saturation $\left(S_{o r}\right)$, and closed symbols are drainages which started from connate water saturation $\left(S_{w c}\right)$. 
a)

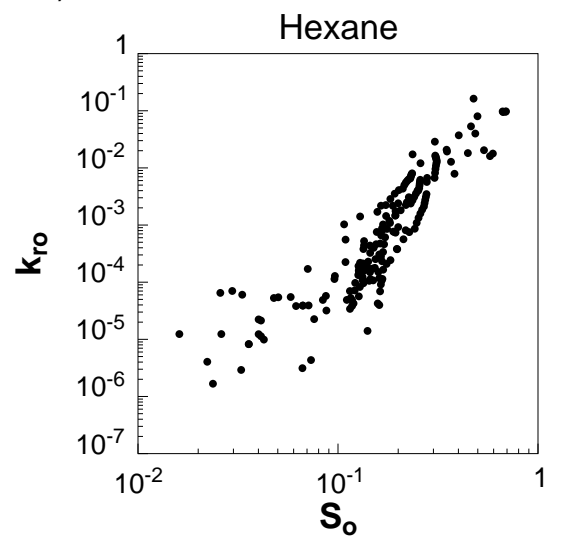

c)

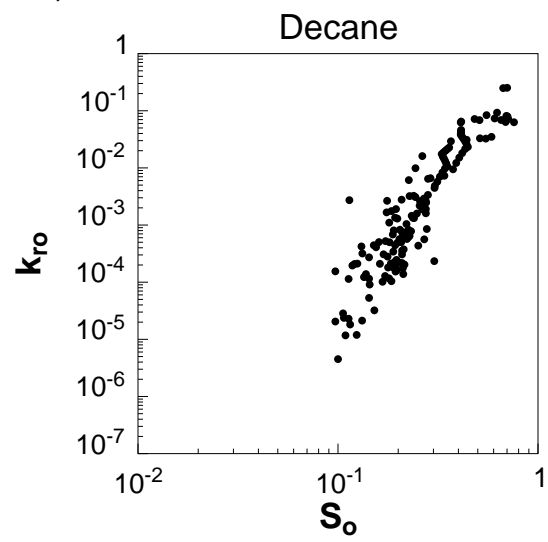

b)

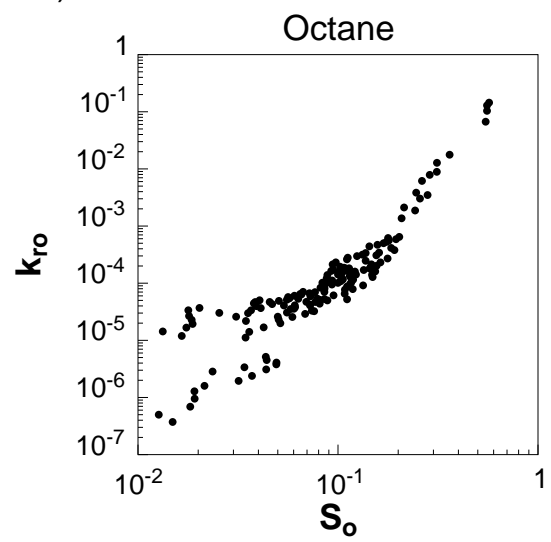

d)

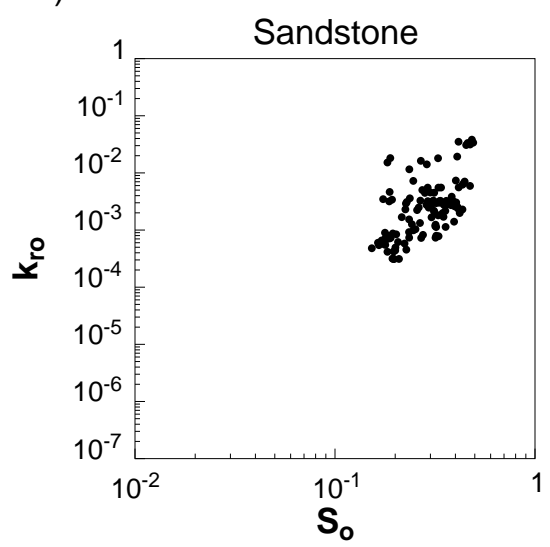

Figure 11.3: Measured oil relative permeabilities with hexane, octane, and decane as the oil in water-wet sand and with octane in water-wet sandstone[186] during three-phase gravity drainage.

Figure 11.2 show the measured relative permeabilities for oil and water for the water-wet, oil-wet, mixed-wet, and fractionally-wet sandpacks with octane as the oil.

The open symbols are from the initial condition of $S_{\text {or }}$ while the closed symbols are from drainages that started from initial condition $S_{w c}$. Note that we can measure saturations as low as $S=0.01$, with relative permeabilities spanning over six orders of magnitude. The observed scatter in the data is likely a result of the combination of uncertainty in the pressure gradient and the natural variations in the porous medium. For ease in viewing the data, each relative permeability (in this figure and the following figures) is shown as a function of only the particular phase's saturation. The relative permeability can also depend on how the rest of the pore space is partitioned between the other two fluids. This can appear as additional scatter in the above plots, and is discussed later.

Figure 11.3 shows the measured oil relative permeability for hexane, octane, and decane in waterwet sand, and for octane in a water-wet consolidated sandstone. Figure 11.4 shows the measured water relative permeability for two-phase (gas/water) and three-phase drainages in water-wet sand. Figure 11.5 shows the measured gas relative permeabilities for a) the two-phase gas/water system for different wettabilities and the two-phase gas/oil system for oil-wet media, and for b) the threephase system for water-wet and oil-wet media. Notice that for the gas permeability the plots are on linear axes. 


\section{Water Relative Permeability}

a)

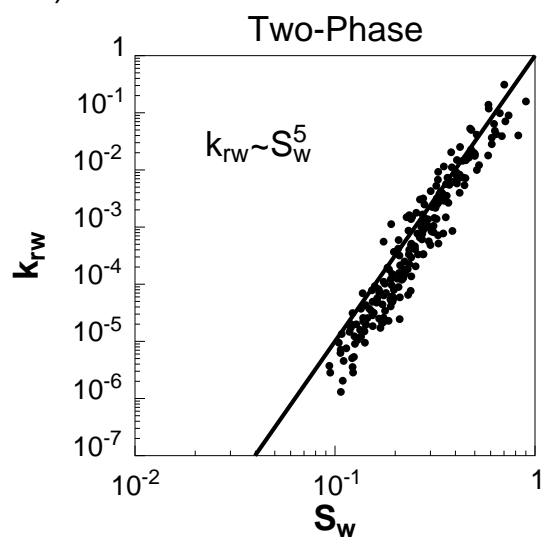

b)

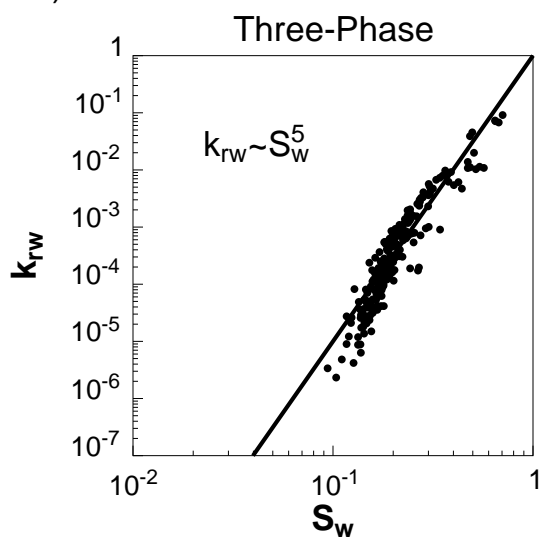

Figure 11.4: Measured water relative permeabilities for a) the two-phase (gas/water) system and for b) the three-phase (gas/oil/water) system from gravity drainages in water-wet sand.

\section{Gas Relative Permeability}

a)

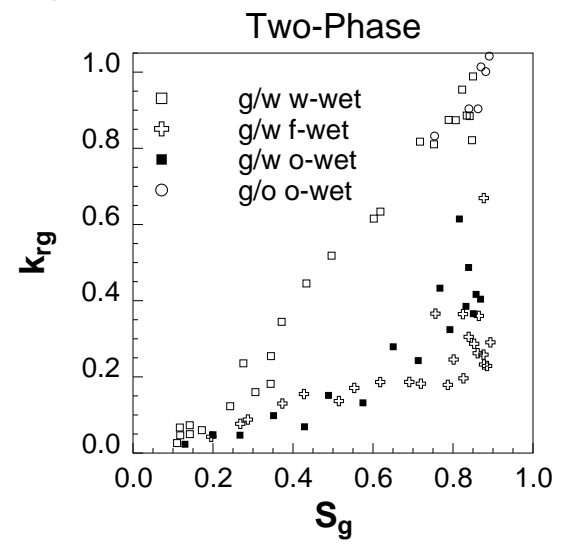

b)

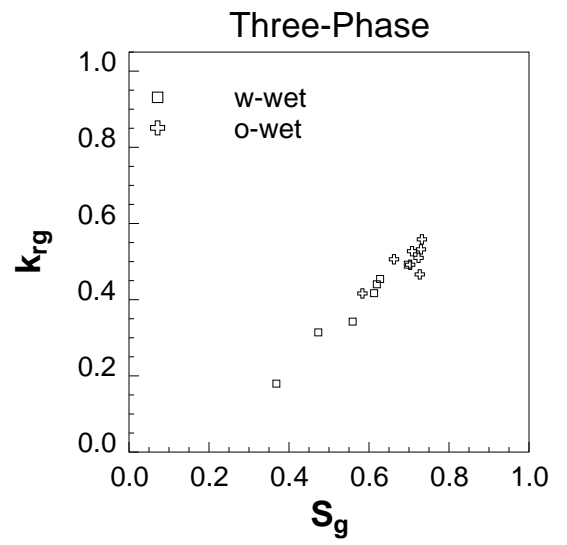

Figure 11.5: Measured gas relative permeabilities for a) the two-phase gas/water system for media of varying wettability and the two-phase gas/oil system for oil-wet media, and for b) the three-phase gas/oil/water system for water-wet and oil-wet media. The gas relative permeability in oil-wet sand is roughly a factor of two smaller than that for identical water-wet sand for two-phase gas/water systems. 
The following features are observed in the measured relative permeabilities:

1. Except for the water in the mixed-wet sand, the oil and water relative permeabilities during three-phase drainage are independent of initial condition to within experimental scatter (Fig. 11.2).

2. The three-phase relative permeabilities of the most wetting fluid (water in water-wet media, oil in oil-wet media) and the two-phase water relative permeability in water-wet media are similar. All can be well described by a simple power law $k_{r} \sim S^{\alpha}$, where $\alpha \approx 5$ (Figs. 11.4a, $11.4 \mathrm{~b}, 11.2 \mathrm{c}$ and $11.2 \mathrm{e}$ ). For the most wetting fluid the permeability is the same for two and three phase displacements. However, for the most wetting phase, a lower saturation is reached in the oil-wet medium (Fig. 11.2e) than in the water-wet medium (Fig. 11.2c).

3. At low saturations, $S<S_{\text {or }}\left(S_{w c}\right)$, the relative permeabilities of oil in water-wet media (Fig. 11.2b) and water in oil-wet media (Fig. 11.2f) are very different. For hexane and octane, the oil relative permeability remains finite at low saturations with a power law of $k_{r} \sim S^{\alpha}$, where $\alpha \approx 2$ (Figs. 11.2b, 11.3a and 11.3b). The water relative permeability drops off quickly, tending to zero at water saturations of $S_{w} \approx 0.1$ (Fig. 11.2f).

4. For the mixed-wet sandpack, the oil relative permeability is similar to the water-wet sandpack at low saturations (Figs. $11.2 \mathrm{~h}$ and $11.2 \mathrm{~b}$ ). It has the largest $k_{\text {ro }}$ of any wettability at moderate saturations $\left(S_{o}=0.2-0.4\right)$. The water relative permeability is the smallest of all the wettabilities, and it depended on the initial condition (Fig. 11.2i).

5. When water is the main liquid phase, the gas relative permeability for the oil-wet and fractionally-wet medium is roughly a factor of two smaller than that for an identical waterwet medium (Fig. 11.5a). When oil is the main liquid phase, the gas relative permeability is independent of the wettability (Fig. 11.5a).

6. For the fractionally-wet sandpack, the oil, water and gas relative permeabilities are between the oil, water and gas relative permeabilities in the water-wet and oil-wet sands (Figs. 11.2k, 11.21 and $11.5 \mathrm{a})$.

Although exact functional forms of relative permeabilities can be dependent on the specific porous medium, we believe that many of the above features are universal for three-phase displacements. We discuss this by considering the effects of pore scale configuration on the relative permeabilities.

\subsection{Pore Scale Explanation}

1. The oil and water relative permeabilities are independent of initial condition during threephase drainage (except for the mixed-wet case).

In water-wet media, the water occupies the smallest pores, regardless of the saturations of oil and gas. Thus in a drainage-type experiment with the water saturation decreasing, the relative permeability of water should be insensitive to initial condition. This behavior has been seen by other researchers $[6,65]$. Oil, however, occupies the intermediate sized pores, and its pore occupancy does depend on the saturations of both water and gas: in a gas/oil displacement, oil occupies the smaller pores, whereas for an oil/water displacement, oil occupies the larger pores. Reviews of the literature have shown that the oil relative permeability is sensitive to initial condition $[6,65]$. We do not see this for $S_{o}>0.05$, and the oil relative permeability appears to be a function only of its own 
saturation. There is a significant effect of initial condition on $k_{r o}$ for $S_{o}<0.05$ in Fig. 11.2b. However, it is difficult to make a definitive comment because of considerable experimental inaccuracies in this low saturation region. The lack of sensitivity to initial condition at moderate oil saturations, could be due to the narrow pore size distribution of the sandpacks, and is consistent with other results in uniform media [76,6]. Similarly, for water in oil-wet media, we see no sensitivity to initial condition, although the water is no longer the wetting phase.

In the mixed-wet media, we do see a measurable dependence on the initial condition for the water relative permeability. The water relative permeability is noticeably larger when starting from $S_{w c}$ then from $S_{o r}$. The water saturation does not drop below $S_{w}=0.15$ when starting from $S_{o r}$, while it reaches less than $S_{w}=0.10$ when starting from $S_{w c}$. When the initial condition is $S_{w c}$, water is only present in the smaller pores and corners. In this sense it resembles a water-wet medium, and as can be seen in Figs. 11.2i and 11.2c, the water relative permeability is similar to the water-wet case. When drainage (gas invasion) starts from $S_{\text {or }}$, water is also present in the centers of larger pores. If these pores are oil-wet, then water can be trapped in them during drainage. In the oil-wet system, a residual water saturation of around $S_{w}=0.10$ is observed (Fig. 11.2f). In the mixed-wet pack, the final water saturation is approximately $S_{w}=0.15$, or the sum of the oil-wet residual and the remaining water saturation in the corners.

2. The relative permeability of the most wetting phase in water-wet and oil-wet media are similar.

The most wetting phase occupies the smallest pores, and corners, grooves, and crevices in the wider pores. If we compare strongly and uniformly water-wet and oil-wet media, we would expect the configuration of the most wetting fluid to be similar for both systems at the same saturation. The exact functional form of the relative permeability depends on the porous medium and is not universal. The only general feature is that $k_{r}$ should asymptotically approach zero saturation at a sufficiently high capillary pressure, indicating that the wetting phase remains connected, albeit poorly, through wetting layers in crevices of the medium down to very low saturation.

In the experiments, the lowest wetting phase saturation observed depends on the largest capillary pressure reached - it takes an infinite capillary pressure to reach zero saturation. It is possible to show [42] that at the top of the column smaller interfacial radii of curvature are achieved for oil in the oil-wet system than for water in the water-wet system. This explains why in our experiments the lowest water saturation reached in the water-wet pack $\left(S_{w} \approx 0.1\right)$ is higher than that reached in the oil-wet pack $\left(S_{o} \approx 0.04\right)$ - see Fig. 11.2.

3. The relative permeability of the intermediate wetting phase (oil in water-wet media, water in oil-wet media) at low saturation are very different.

At low saturation phases may remain connected through wetting layers in crevices in the pore space. It is this connectivity which controls $k_{r}$ at low saturation. The pore scale configuration and connectivity of oil and water is very different for water-wet and oil-wet media. Consider first flat water-wet and oil-wet surfaces as shown in Figure 11.6.

The most wetting fluid coats the surface. The contact angle between oil or water and gas, which will control the position of the phase in the pore space, is given by the balance of interfacial tensions, assuming that the solid is coated with a thick wetting film.

For oil in water-wet media, (Fig. 11.6a)

$$
\cos \theta_{g o}=\frac{\gamma_{g w}-\gamma_{o w}}{\gamma_{g o}}=1+\frac{C_{s o}}{\gamma_{g o}},
$$

where

$$
C_{s o}=\gamma_{g w}-\gamma_{o w}-\gamma_{g o}
$$


a)

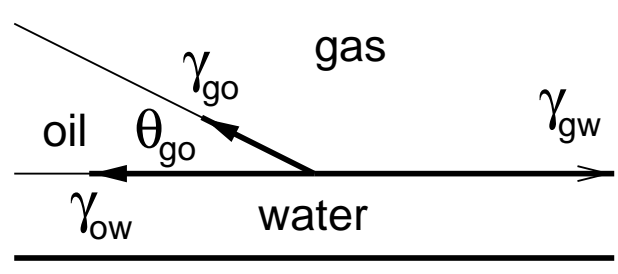

water-wet surface b)

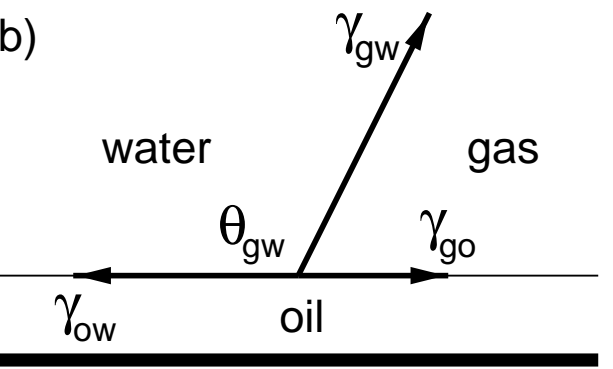

oil-wet surface

Figure 11.6: Configuration of fluids on : a) a flat water-wet surface, b) a flat oil-wet surface (shown bold).
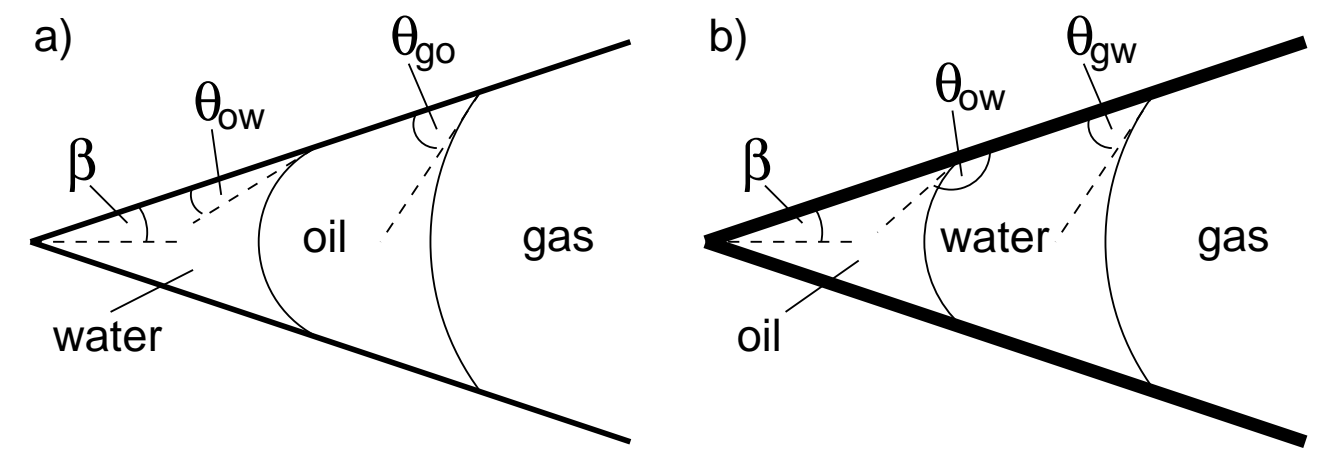

Figure 11.7: Possible configuration of fluids in a pore space corner of half-angle $\beta$ : a) water-wet surfaces, b) oil-wet surfaces (shown bold). Note that configuration b) is not allowed as $\theta_{g w}>90^{\circ}$.

is the spreading coefficient for oil. Using our data in Table 11.1 we find $C_{s o}=3.5,-0.3$, and -3.1 $\mathrm{mN} / \mathrm{m}$ for hexane, octane, and decane, respectively. For octane (the most used fluid) this results in $\theta_{g o} \approx 10^{\circ}$.

Now consider the three fluids in a corner of the pore space as shown in Fig. 11.7a. If $\theta_{\text {ow }}+\beta<90^{\circ}$ and $\theta_{g o}+\beta<90^{\circ}$, where $\beta$ is the half-angle of the corner, then a layer of oil may be present $[160,68]$. These layers are on the order of micrometers thick and have been observed in micromodel experiments $[156,196,115]$. Drainage through oil layers is the mechanism by which very low oil saturations are reached during gas displacement in water-wet media [49, 114, 227]. Final oil saturations as low as $S_{o}=0.001$ have been reached [227]. For a system with $\theta_{\text {ow }}=\theta_{\text {go }}=0$, the oil layers can drain to infinitesimal thickness at a finite gas/oil capillary pressure, meaning that in theory, $S_{o}=0$ can be achieved through gravity drainage[19,227]. In our experiments the lowest saturation obtained is $S_{o} \approx 0.01$ after 7 weeks of drainage.

Displacement of oil below the waterflood residual oil saturation, $S_{o r}$, requires layer drainage. In our experiments, and others on bead packs, sandpacks and consolidated sandstones a layer drainage regime consistent with $k_{r o} \sim S_{o}^{2}$ has been observed for $S_{o}<S_{o r}$ (see Figs. 11.3a, 11.3b, and 11.9a) [186, 147, 76]. This is readily explained by examining the flow in a single angular pore (see Fig. 11.7a). $S_{o}$ is proportional to the area of the oil in the layer. The oil conductance for Poiseuille type flow is approximately proportional to the area squared, leading to $k_{r o} \sim S_{o}^{2}[68,229]$. This may seem a simple argument, but it is confirmed by more detailed analysis of layer flow that has been verified against numerical solutions of the Navier Stokes equation and experiments in 
square[229] and triangular[70] capillary tubes.

We observe layer drainage for a spreading system - loosely defined as one with $C_{s o} \approx 0$, or $\theta_{g o} \approx 0$. However, the situation is different for oils with a large and negative spreading coefficient. For decane in water-wet media, our measurements of interfacial tension result in $\theta_{g o} \approx 30^{\circ}$. Decane layers have been seen in micromodels [115]. However, decane layers cannot form in oblique wedges $\left(\beta>60^{\circ}\right)$, and are present for a more restricted range of capillary pressures than for octane [68]. If oil layers are not present, oil can be trapped, leaving a residual saturation at the end of drainage. In our experiments we do not observe a layer drainage regime, as shown in Fig. 11.9b.

For water in strongly oil-wet media, (Fig. 11.6b)

$$
\cos \theta_{g w}=\frac{\gamma_{g o}-\gamma_{o w}}{\gamma_{g w}}=1+\frac{C_{s w}}{\gamma_{g w}},
$$

where

$$
C_{s w}=\gamma_{g o}-\gamma_{o w}-\gamma_{g w}
$$

is the spreading coefficient for water. Using Table 11.1 we find $C_{s w}=-102.1 \mathrm{mN} / \mathrm{m}$ and $\theta_{g w} \approx 115^{\circ}$. Even if the surface is not strongly oil-wet, there is a constraint between the interfacial tensions and contact angles[229],

$$
\gamma_{g w} \cos \theta_{g w}=\gamma_{o w} \cos \theta_{o w}+\gamma_{g o} \cos \theta_{g o}
$$

Using the octane data, if $\theta_{o w}>110^{\circ}$ then $\theta_{g w}>90^{\circ}$. Thus water is less wetting than gas, unless we have a very weakly oil-wet system $\left(\theta_{\text {ow }}<110^{\circ}\right)$.

Measurements on oil-treated glass surfaces have found $\theta_{g w}=103^{\circ}$, consistent with Eq. 11.9. In Fig. 11.7b, water layers can occur for $\theta_{g w}+\beta<90^{\circ}$, and gas layers can occur for $90^{\circ}+\beta<\theta_{g w}$, neither of which are possible except for gas in exceptionally sharp crevices. The absence of a layer drainage regime is evident in Fig. 11.9c.

Note that $k_{r o}$ for decane in a water-wet system is similar to $k_{r w}$ in an oil-wet system. Both represent cases where the intermediate-wet fluid is nonspreading and there is no layer drainage regime.

We do not observe the same layer drainage regime for the most wetting phase - instead of $k_{r} \sim S^{2}$, we observe $k_{r} \sim S^{5}$. The most wetting phase occupies the small pores and all the narrow nooks and crannies, where it is held by strong capillary forces and where it may be poorly connected. In contrast, oil layers in spreading systems reside over the water, and by construction therefore, are well connected throughout the porous medium.

4. The mixed-wet sandpack has an oil relative permeability similar to the water-wet pack at low saturations, while it was the largest of any wettability at moderate saturations $\left(S_{o}=0.2-0.4\right)$. The water relative permeability is the smallest observed for any wettability.

Figure 11.8 shows schematic arrangements of oil, water and gas in water-wet, oil-wet, and mixedwet pores. In both water-wet and mixed-wet media water fills the corners of the pore space, even if oil fills the pore center. During gas injection, gas is nonwetting to oil, and thus in both cases, layers of oil between water in the corners and gas in the pore centers may be present. As a consequence, $k_{r o}$ at low $S_{o}$ is similar for mixed-wet and water-wet media, with a characteristic layer drainage regime. This implies a possible universal form for $k_{r o}$ at low $S_{o}$ in mixed-wet reservoir settings, since the behavior is independent of the oil/water contact angle. Notice that the layers can form in a mixed-wet system, even if water is in the center of the pores. This agrees with the observations that $k_{r o}$ at low saturations is independent of the initial condition even when more water is trapped in the pores when starting from $S_{\text {or }}$. In contrast, in uniformly oil-wet media, oil is confined to the corners. The oil is held by strong capillary forces and has a lower $k_{r o}$ as discussed before. 


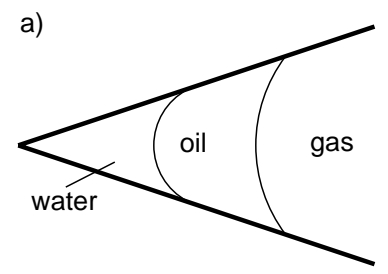

water-wet

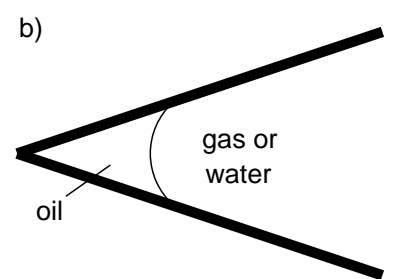

oil-wet

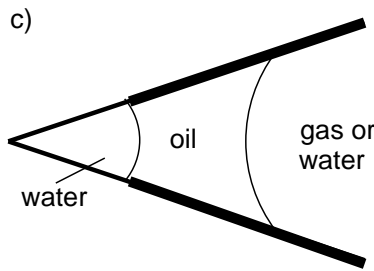

mixed-wet

Figure 11.8: Pore-scale arrangements of fluid for water-wet, oil-wet, and mixed-wet corners. A thick line represents an oil-wet surface. Notice that oil layers sandwiched between water and gas are present for both the water-wet and mixed-wet media, leading to similar $k_{r o}$ at low $S_{o}$. In oil-wet media, oil is confined to the crevices, leading to a lower $k_{r o}$.

Although it is difficult to see from the log-log plots of Fig. 11.2, between $S_{o}=0.2-0.4, k_{r o}$ was noticeably the largest in the mixed-wet packs. In this saturation range, the fluids will be contacting both oil-wet and water-wet surfaces. This being said, it seems most likely that $k_{r o}$ would be smaller than the water-wet case, as the oil is most wetting on some of these surfaces. Thus, there is not a simple explanation of this phenomena, although it would be interesting what behavior is seen in a three-phase, mixed-wet network model.

The low water relative permeability is due to the large trapped water saturation during drainage. As explained in item 1, this is greatest in mixed-wet packs, as the water can get trapped in the big pores, and held by capillary forces in the small pores.

5. When water is the main liquid phase, The gas relative permeabiltiy is lower in an oil-wet medium than in a water-wet medium.

For the two-phase gas/water system in water-wet media, the water phase will occupy the smallest pores and crevices while the gas phase occupies the large pore spaces. In oil-wet media, the contact angle is such that neither the water phase nor the gas phase wets the pore surfaces and thus the water and gas phases compete for the largest pores. Thus at equivalent gas saturations, in the oil-wet system the gas is in smaller pathways leading to a lower permeability.

For the three-phase gas/oil/water system in water-wet media, the gas is nonwetting to both oil and water, and so $k_{r g}$ is expected to be a function only of gas saturation. In oil-wet media, gas is nonwetting to oil, but is not strongly nonwetting to water. Thus it is expected that in this case $k_{r g}$ depends on both the oil and water saturations. From Fig. $11.5 \mathrm{~b}$ we see that the three-phase $k_{r g}$ in the oil-wet system is between the two-phase gas/water $k_{r g}$ and gas/oil $k_{r g}$. Most three-phase models [200, 201, 65, 6] assume that gas is always the nonwetting phase and is a function of gas saturation only, which is not supported by these measurements.

We also see that the three-phase $k_{r g}$ in the water-wet system is lower than the two-phase waterwet gas/water $k_{r g}$ and oil-wet gas/oil $k_{r g}$. Since the gas is the most nonwetting phase in all of these experiments, we expect the gas relative permeabilities to be similar. Why this is not the case is currently unknown.

6. The fractionally-wet sand has behavior intermediate between oil-wet and water-wet sand.

This last observation seems intuitively obvious. However, there are three important points to make. First, the oil in a spreading system is always connected regardless of the oil-wet fraction, 
a)

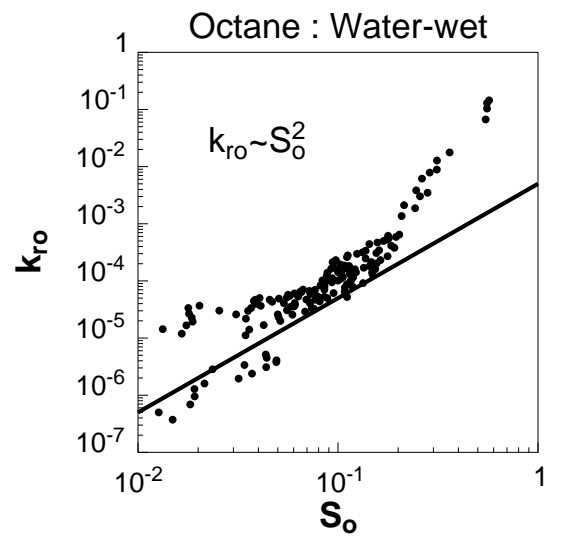

C)

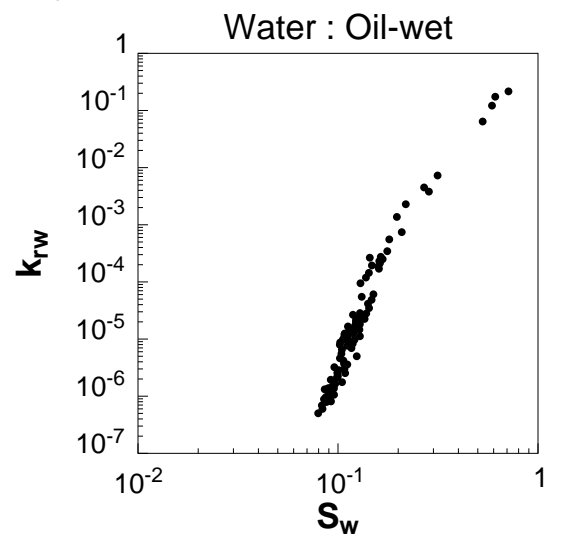

b)

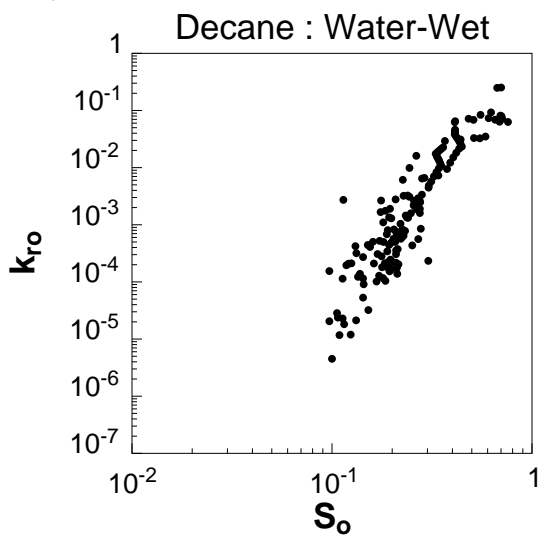

d)

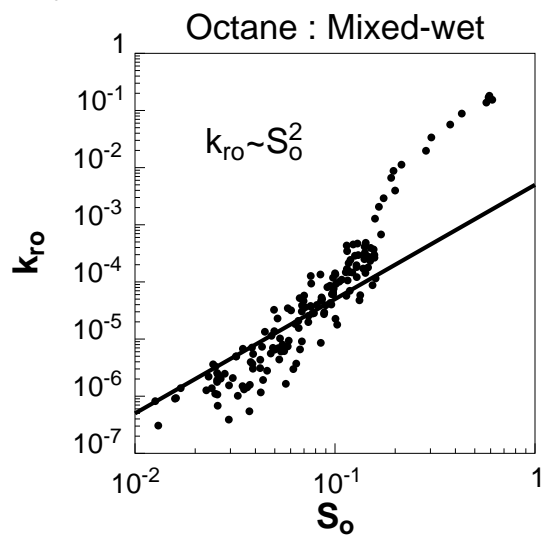

Figure 11.9: Comparison of relative permeabilities. (a) A spreading system $\left(\theta_{g o} \approx 0\right)$ : Octane in water-wet sand. (b) A nonspreading system : Decane in water-wet sand. (c) A nonwetting system : Water in oil-wet sand. (d) A mixed-wet system : Octane in mixed-wet sand. The solid lines in (a) and (d) show $k_{r o} \sim S_{o}{ }^{2}$. The data, while consistent with this trend, only follows it approximately. 
since it can reside in corners as the most wetting phase or in layers as the intermediate wetting phase. Consistent with this is the observation that $k_{r o}$ asymptotically approaches zero residual oil saturation, for all wettabilities, as shown in Fig. 11.2. However, unless the small pores, corners and crevices are completely water-wet, an infinite capillary pressure is required to achieve this. Second, note that the trapped water saturation for the fractionally-wet sand is slightly higher than for the oil-wet sand. This is because in the fractionally-wet sand, the oil-wet regions are poorly connected in the pack leading to significant trapping of water. This percolation-type argument has been explored in more detail by Zhou et al. [228]. Third, the gas relative permeability for the fractionally-wet sand is very close to the oil-wet case. This shows that the gas mobility is significantly affected even if a fraction of the pores are oil-wet.

\subsection{Discussion}

Recently, there has been renewed interest in predicting capillary pressure curves and relative permeabilities from basic physics and the pore structure using pore network models (see, for instance [157]). One of our objectives in this study was to obtain a complete data set (capillary pressure curves and relative permeabilities) as a function of wettability. We hope that this data can provide a benchmark with which to test the various three-phase, mixed-wettability network models now being proposed. In this vein, the data can all be found by following links from our web site: http://ekofisk.stanford.edu/supric.html/ .

\subsection{Conclusions}

We used CT scanning and analogue experiments to measure oil, water and gas relative permeabilities during three-phase gravity drainage. Mixed-wet sandpacks were made by flooding a water-filled, water-wet pack with crude oil. The capillary pressure curve of the resultant medium indicated that it was neither strongly oil-wet nor water-wet. Drainage experiments were performed on oil-wet, water-wet, mixed-wet, and fractionally-wet sandpacks, Saturations as low as 0.01 were recorded and the relative permeabilities span six orders of magnitude. The main results are:

1. At low oil saturations $\left(S_{o}<S_{o r}\right)$ in water-wet media, spreading systems show a characteristic form of the oil relative permeability with approximately $k_{r o} \sim S_{o}{ }^{2}$. This behavior is consistent with a theoretical interpretation of oil layer drainage at the pore scale. For a nonspreading decane system, the layer drainage regime was not observed.

2. Mixed-wet media also exhibit an approximate quadratic oil relative permeability at low oil saturations. The theoretical interpretation is that oil layers are present between water on the water-wet corners of the pore space, and gas occupies the oil-wet center.

3. In oil-wet media, the oil relative permeability is similar to the water relative permeability in water-wet systems. This behavior is consistent with the interpretation that the most-wetting phase occupies the same portion of the pore space in either wetting situation.

4. In oil-wet media, the water has a large and negative spreading coefficient, meaning that water layers do not form in the pore space. The water relative permeability $k_{r w}$ for an oil-wet medium is similar to $k_{r o}$ for a water-wet medium and a nonspreading oil.

5. In oil-wet media, the gas relative permeability is smaller than that for identical water-wet media. This behavior is consistent with the gas and water phases competing for the largest pores in oil-wet media, where gas is not necessarily the nonwetting phase. 


\subsection{Nomenclature}

$C_{s o}=$ spreading coefficient for oil

$C_{s w}=$ spreading coefficient for water

$I_{o}=$ Amott oil index

$I_{w}=$ Amott water index

$g=$ gravitational constant

$k_{r i}=$ relative permeability to phase $i$

$P_{i}=$ pressure of phase $i$

$S_{i}=$ saturation of phase $i$

$S_{o r}=$ waterflood residual oil saturation

$S_{w c}=$ connate water saturation

$u_{i}=$ flux of phase $i$

\section{Greek Letters}

$\alpha=$ power law exponent

$\beta=$ half angle of pore corner

$\gamma_{\text {ow }}=$ oil/water interfacial tension

$\gamma_{g o}=$ gas $/$ oil interfacial tension

$\gamma_{g w}=$ gas $/$ water interfacial tension

$\mu_{i}=$ viscosity of fluid $i$

$\phi \quad=$ porosity

$\rho_{i}=$ density of fluid $i$

$\theta_{\text {ow }}=$ oil $/$ water contact angle

$\theta_{g o}=$ gas $/$ oil contact angle

$\theta_{g w}=$ gas $/$ water contact angle

\section{Subscripts}

$i=$ phase

$g=$ gas

$o \quad=$ oil

$w \quad=\quad$ water (brine) 


\section{References}

[1] Adamson, A.W.: Physical Chemistry of Surfaces, John Wiley and Sons, NY, NY (1990).

[2] Akin, S. and Demiral, M.R.B.: "Effect of Flow Rate on Imbibition Three-Phase Relative Permeabilities and Capillary Pressure," in the proceedings of the .

[3] Amott, E.: "Observations Relating to the Wettability of a Porous Rock," Trans. AIME (1959) 216, 156-162.

[4] Anderson, E., Bai, Z., Bischof, C., Demmel, J., Dongarra, J., Croz, J. D., Greenbaum, A., Hammarling, S., McKenney, A., Ostrouchov, S., and Sorensen, D.: LAPACK Users' Guide, Siam (1992).

[5] Aziz, K. and Settari, A.: Petroleum Reservoir Simulation, Applied Science Publishers, London (1979).

[6] Baker, L.E.: "Three-Phase Relative Permeability Correlations," paper SPE 17369 in the proceedings of the 1988 Sixth SPE/DOE Symposium on Enhanced Oil Recovery, Tulsa, OK, April.

[7] Bakke, S. and Øren, P.E.: "3-D Pore-Scale Modelling of Sandstones and Flow Simulations in the Pore Networks," SPE Journal (1997) 1, 136.

[8] Barker, J.W. and Fayers, F.J.: "Transport Coefficients for Compositional Simulation with Coarse Grids in Heterogeneous Media," SPE Advanced Technology Series (April 1994) 2, No. 2, 103-113.

[9] Barker, J.W. and Thibeau, S.: "A Critical Review of the Use of Pseudorelative Permeabilities for Upscaling," SPE Reservoir Engineering (May 1997) 12, No. 2, 138-143.

[10] Batycky, R.P., Blunt, M.J., and Thiele, M.R.: “A 3D Field Scale Streamline Simulator With Gravity and Changing Well Conditions," SPE Reservoir Engineering (November 1997) 11, No. 4.

[11] Batycky, R.P., Thiele, M.R., and Blunt, M.J.: "A Streamline Simulator to Model Field Scale, Three-Dimensional Flow," Proc. of the 5th European Conference on the Mathematics of Oil Recovery, Leoben, Austria (Sept. 3-6 1996).

[12] Batycky, R.P., Thiele, M.R., and Blunt, M.J.: "A Streamline-Based Reservoir Simulation of the House Mountain Waterflood," Stanford Center for Reservoir Forecasting (SCRF) Annual Report, Stanford University (1997).

[13] Begg, S., Carter, R., and Dranfield, P.: “Assigning Effective Values to Simulator Gridblocks Parameters for Heterogeneous Reservoirs," SPE Reservoir Engineering (1989) 4, 455-463. 
[14] Behrens, R.A., Jones, R.C., and Emanuel, A.S.: "Implementation of a Streamline Method for Flow Simulation of Large Fields," In Proceedings of 47th Annual Technical Meeting of the Petroleum Society, Calgary, Canada, CIM 96-55 (June 10-12 1996).

[15] Benham, A.L., Dowden, W.E. and Kunzman, W.J.: "Miscible Fluid Displacement - Prediction of Miscibility," Trans. AIME (1959) 216, 388-398.

[16] Blunt, M.J.: "Effects of Heterogeneity and Wetting on Relative Permeability Using Pore Level Modeling," SPE Journal (1997) 1, 70-87.

[17] Blunt, M.J. and Rubin, B.: "Implicit Flux Limiting Schemes for Petroleum Reservoir Simulation," Journal of Computational Physics (September 1992) 102, No. 1, 194-210.

[18] Blunt, M.J., Liu, K., and Thiele, M.R.: "A Generalized Streamline Method to Predict Reservoir Flow," Petroleum Geosciences (August 1996) 2, 259-269.

[19] Blunt, M.J., Zhou, D. and Fenwick, D.H.: "Three-Phase Flow and Gravity Drainage in Porous Media," Transport in Porous Media (1995) 20, 77-103.

[20] Bommer, M.P. and Schechter, R.S.: "Mathematical Modeling of In-Situ Uranium Leaching," Society of Petroleum Engineers Journal (December 1979) 19, 393-400.

[21] Bratvedt, F., Bratvedt, K., Buchholz, C.F., Holden, L., Holden, H., and Risebro, N.H.: "A New Front-Tracking Method for Reservoir Simulation," SPE Reservoir Engineering (February 1992) $7,107-116$.

[22] Bratvedt, F., Gimse, T., and Tegnander, C.: "Streamline Computations for Porous Media Flow Including Gravity," Transport in Porous Media (October 1996) 25, 63-78.

[23] Bryant, S. and Blunt, M.J.: "Prediction of Relative Permeability in Simple Porous Media," Physical Review A (August 1992) 46, 2004-2011.

[24] Buckley, J.S. and Liu, Y.: "Some Mechanisms of Crude Oil/Brine/Solid Interactions," Journal of Petroleum Science and Engineering (1998) 20, 155-160.

[25] Buckley, J.S., Liu, Y., Xie, X. and Morrow, N.R.: "Asphaltenes and Crude Oil Wetting The Effect of Oil Composition," SPE Journal (1997) 2, 107-119.

[26] Burger, J. and Mohanty, K.: "Mass Transfer From Bypassed Zones During Gas Injection," SPE Reservoir Engineering (May 1997) 12, No. 2, 124-130.

[27] Burger, J.E., Bhogeswara, R., and Mohanty, K.K.: "Effect of Phase Behavior on Bypassing in Enriched Gasfloods," SPE Reservoir Engineering (May 1994) 9, No. 2, 112-118.

[28] Chalier, G., Giry, V., Madaoui, K., Sakthikumar S., and Maquignon Ph.: "Three-Phase Oil Relative Permeability Determination as a Key Point in the Evaluation of a Tertiary Gas Gravity Drainage Project," paper SPE 30761 in the proceedings of the 1995 SPE Annual Technical Conference and Exhibition, Dallas, TX, October.

[29] Chang, Y.-B., Lim, M.T., Pope, G.A., and Sepehrnoori, K.: " $\mathrm{CO}_{2}$ Flow Patterns Under Multiphase Flow: Heterogeneous Field Scale Conditions," SPE Reservoir Engineering (August 1994) 9, No. 3, 208-216. 
[30] Chatzis, I., Kantzas, A., and Dullien, F.A.L.: "On the Investigation of Gravity Inert Gas Injection Using Micromodels, Long Berea Cores, and Computer Assisted Tomography," paper SPE 18284 in the proceedings of the 1988 SPE Annual Technical Conference and Exhibition, Houston, TX, October.

[31] Christie, M.: "Upscaling for Reservoir Simulation," Journal of Petroleum Technology (Nov 1996) 48, 1004-1008.

[32] Christie, M. and Bond, D.: "Detailed Simulation of Unstable Processes in Miscible Flooding," SPE Reservoir Engineering (November 1987) 2, No. 4, 514-522.

[33] Christie, M.A.: "Upscaling for Reservoir Simulation," Journal of Petroleum Technology (November 1996) 48, No. 11, 1004-1010.

[34] Christie, M.A. and Clifford, P.J.: "A Fast Procedure for Upscaling in Compositional Simulation," paper SPE 37986 in the proceedings of the 1997 14th Symposium on Reservoir Simulation, Dallas, TX, June.

[35] Colella, P., Concus, P., Sethian, J.: "Some Numerical Methods for Discontinuous Flows in Porous Media," The Mathematics of Reservoir Simulation, Ewing, R. (ed.), SIAM Philadelphia (1983) 161-186.

[36] Corey, A.T., Rajthens, C.H., Henderson, J.H., and Wyllie, M.R.J.: "Three-Phase Relative Permeability Models," Trans. AIME (1956) 207, 349-351.

[37] CRC Press, Inc., West Palm Beach, Florida, CRC Handbook of Chemistry and Physics, 61st edition (1981).

[38] Datta-Gupta, A. and King, M.J.: "A Semianalytic Approach to Tracer Flow Modeling in Heterogeneous Permeable Media," Advances in Water Resources (1995) 18, 9-24.

[39] Del Cerro, C. and Jameson, G.J.: "The Behavior of Pentane, Hexane, and Heptane on Water," J. Colloid 6 Interface Sci. (1980) 78, 362-375.

[40] Delshad, M., and Pope, G.A.: "Comparison of the Three-Phase Oil Relative Permeability Models," Transport in Porous Media (1989) 4, 59-83.

[41] Deutsch, C.V. and Journel, A.G.: GSLIB Geostatistical Software Library and User's Guide, Oxford University Press, New York, NY (1992).

[42] DiCarlo, D.A., Sahni, A., and Blunt, M.J.: "The Effect of Wettability on Three-Phase Relative Permeability," Transport in Porous Media (2000) 39, 347-366.

[43] Dindoruk, B.: Analytical Theory of Multiphase, Multicomponent Displacement in Porous Media, PhD dissertation, Stanford University, Stanford, CA (1992).

[44] Dindoruk, B., Johns, R.T. and Orr, F.M., Jr.: "Analytical Solution for Four Component Gas Displacements with Volume Change on Mixing," (June 1992) Third European Conference on the Mathematics of Oil Recovery, Delft, The Netherlands.

[45] Dindoruk, B., Orr, F.M., Jr. and Johns, R.T.: “Theory of Multicontact Miscible Displacement with Nitrogen," SPE Journal (1997) 2, 268-279.

[46] Dixit, A.B., McDougall, S.R. and Sorbie, K.S.: "A Pore-Level Investigation of Relative Permeability Hysteresis in Water-Wet Systems," paper SPE 37233 in the proceedings of the 1997 SPE International Symposium on Oilfield Chemistry, Houston, TX, February. 
[47] Dong, M., Dullien, F.A.L., and Chatzis, I.: "Imbibition of Oil in Film Form Over Water Present in Edges of Capillaries with an Angular Cross-Section," J. Colloid and Interface Sci. (1995) 172, 278-288.

[48] Dullien, F.: Porous Media: Fluid Transport and Pore Structure, Academic Press, San Diego, CA (1992).

[49] Dumoré, J.M. and Schols, R.S.: "Drainage Capillary Functions and the Influence of Connate Water," SPE Journal (1974) 14, 437-444.

[50] Dumoré, J.M. Hagoort, J. and Risseeuw, A.S.: “An Analytical Model for One-Dimensional, Three-Component Condensing and Vaporizing Gas Drives," SPE Journal (April 1984) 24, 169-179.

[51] Ehlig-Economides, C. and Spath, J.: "Waterflood Infill Well Pattern Strategies for Horizontal and Multibranch Wells," paper SPE 35209 in the proceedings of the 1996 Permian Basin Oil Recovery Conference, Midland, TX.

[52] Emanuel, A.S., Alameda, G.K., Behrens, R.A., and Hewett, T.A.: "Reservoir Performance Prediction Methods Based on Fractal Geostatistics," SPE Reservoir Engineering (August 1989) 4, No. 3, 311-318.

[53] Emanuel, A.S. and Milliken, W.J. : "The Application of Streamtube Techniques to Full Field Waterflood Simulations," paper SPE 30758 in the proceedings of the 1995 70th Annual Fall Technical Conference and Exhibition, Dallas, TX.

[54] Emanuel, A.S., Tang, R.W., McKay, D.M., and Ellis, M.H.: “A Hybrid Simulation Study of the Windalia Sand Waterflood," paper SPE 26477 in the proceedings of the 1993 68th Annual Fall Technical Conference and Exhibition, Houston, TX.

[55] Epic Consulting Services Ltd.: "Shell Canada Limited House Mountain Simulation Study In house report," (January 1997).

[56] Espie, A.A., Brown, C.E., Merrill, R.C., and Newley, T.M.J.: "An Evaluation of Oil Recovery by Vaporisation," paper SPE 27812 in the proceedings of the 1994 DOE/SPE Improved Oil Recovery Symposium, Tulsa, OK, April 17-20.

[57] Espie, A.A., Brown, C.E., Narayanan, K.R., and Wilcox, T.C.: "A Laboratory Investigation of Gravity Drainage/Waterflood Interaction in Prudhoe Bay," paper SPE 28614 in the proceedings of the 1989 SPE Annual Technical Conference and Exhibition, New Orleans, LA, October.

[58] Ewing, R. E.: "Finite element techniques for convective-diffusive transport in porous media," Advances in Water Resources (Sept 1988) 11, No. 3, 123-126.

[59] Falls, A.H. and Schulte, W.M.: "Features of Three Component, Three Phase Displacement in Porous Media," SPE Reservoir Engineering (1992) 7, 426-432.

[60] Falls, A.H. and Schulte, W.M.: "Theory of Three Component, Three Phase Displacement in Porous Media," SPE Reservoir Engineering (1992) 7, 377-384.

[61] Fay, C.H. and Prats. M.: "The Application of Numerical Methods to Cycling and Flooding Problems," Proceedings of the 3rd World Petroleum Congress (1951). 
[62] Fayers, F. and Lee, S.: "Crossflow Mechanisms In Oil Displacements By Gas Drive In Heterogeneous Reservoirs," In Situ (1994) 18, No. 4.

[63] Fayers, F. and Muggeridge, A.: "Extensions to Dietz Theory and Behaviour of Gravity tongues in Slightly Tilted Reservoirs," SPERE (Nov 1990) 5, 487-494.

[64] Fayers, F.J.: "Extension of Stone's Method 1 and Conditions for Real Characteristics in Three-Phase Flow," SPE Reservoir Engineering (1989) 4, 437-445.

[65] Fayers, F.J. and Matthews J.D.: "Evaluation of Normalized Stone's Methods for Estimating Three-Phase Relative Permeabilities," SPE Journal (1984) 24, 224-232.

[66] Fenwick, D.H.: Pore Scale Modeling of Three-Phase Flow, PhD dissertation, Stanford University, Stanford, CA (February 1997).

[67] Fenwick, D.H., and Blunt, M.J.: "Calculating Three-Phase Relative Permeabilities Using Network Modeling," proceedings of the 1996 5th European Conference on the Mathematics of Oil Recovery, Leoben, Austria (Sept. 1996).

[68] Fenwick, D.H. and Blunt, M.J.: "Network Modeling of Three-Phase Flow in Porous Media," SPE Journal (1998) 3, 86-97.

[69] Fenwick, D.H. and Blunt, M.J.: "Three-Dimensional Modeling of Three-Phase Imbibition and Drainage," Advances in Water Resources (1998) 21, 121-144.

[70] Firincioglu, T., Blunt, M.J. and Zhou, D.: "Three-Phase Flow and Wettability Effects in Triangular Capillaries," Colloids and Surfaces A (1999) 155, 259-276.

[71] Gautier, Y. and Notinger, B.: "Preferential flow-paths detection for heterogeneous reservoir using a new renormalization technique," Transport in Porous Media (1997) 26, 1-23.

[72] GeoQuest Reservoir Technologies: Eclipse 300 Reference Manual - 96A Release, Schlumberger GeoQuest, 5599 San Felipe, Suite 1700, Houston, TX 77056 (1996).

[73] Glaso, O.: "Generalized Minimum Miscibility Pressure Correlation," SPE Journal (1985) 25, 927-934.

[74] Glimm, J., Lindquist, B., McBryan, O.A., Plohr, B., and Yaniv, S.: "Front Tracking for Petroleum Simulation," paper SPE 12238 in the proceedings of the 1983 Reservoir Simulation Symposium, San Francisco, CA, November 15-18.

[75] Goodyear, S.G., and Jones, P.I.R.: "Relative Permeabilities for Gravity Stabilized Gas Injection," proceedings of the 8th European IOR Symposium in Vienna, Austria (May 15-17 1995).

[76] Grader, A.S., and O’Meara, D.J., Jr.: "Dynamic Displacement Measurements of Three-Phase Relative Permeabilities Using Three Immiscible Liquids," paper SPE 18293 in the proceedings of the 1992 SPE Annual Technical Conference and Exhibition, Houston, TX, Oct. 2-5.

[77] Guedes, S. and Schiozer, D.: "An Implicit Treatment of Upscaling in Numerical Simulation," SPE Symposium on Reservoir Simulation, Houston (1999) No. 51937.

[78] Guérillot, D. and Verdière, S.: "Different Pressure Grids for Reservoir Simulation in Heterogeneous Reservoirs," SPE Symposium on Reservoir Simulation, San Antonio, TX (1995) No. 29148. 
[79] Guzman, R.E.: Mathematics of Three Phase Flow, PhD dissertation, Stanford University, Stanford, CA (July 1995).

[80] Guzman, R.E. and Fayers, F.J.: "Mathematical Properties of Three-Phase Flow Equations," SPE Journal (1997) 2, 291-300.

[81] Guzman, R.E. and Fayers, F.J.: "Solution to the Three-Phase Buckley-Leverett Problem," SPE Journal (1997) 2, 301-311.

[82] Hansen, R. and Espedal, M.S.: "On the Numerical Solution of Non-Linear Reservoir Flow Models with Gravity," International Journal for Numerical Methods in Engineering (June 1995) 38, No. 12, 2017-2032.

[83] Hearn, C.L. and Whitson, C.H.: "Evaluating Miscible and Immiscible Gas Injection in the Sarah Field, Oman," paper SPE 29115 in the proceedings of the 1995 13th SPE Symposium on Reservoir Simulation, San Antonio, TX.

[84] Heiba, A.A., Davis, H.T. and Scriven, L.E.: "Statistical Network Theory of Three-Phase Relative Permeabilities," paper SPE 12690 in the proceedings of the 1984 4th DOE/SPE Symposium on Enhanced Oil Recovery, Tulsa, OK, April.

[85] Helfferich, F.G.: "Theory of Multicomponent, Multiphase Displacement in Porous Media," SPE Journal (February 1981) 271, 51-62.

[86] Hewett, T. and Behrens, R.: "Scaling Laws in Reservoir Simulation and Their Use in a Hybrid Finite Difference/Streamtube Approach to Simulation the Effects of Permeability Heterogeneity," Reservoir Characterization, II, L. Lake and J. Carroll, H.B. (eds.), Academic Press, Inc., London (1991) 402-441.

[87] Hewett, T.A. and Behrens, R.A.: "Conditional Simulation of Reservoir Heterogeneity With Fractals," SPE Reservoir Engineering (September 1990) 8, 217-225.

[88] Hewett, T.A. and Yamada, T.: "Theory of the Semi-Analytical Calculation of Oil Recovery and Effective Relative Permeabilities using Streamtubes," Advances in Water Resources (1996) 20, 279-292.

[89] Hicks, P.J., Jr.: "X-Ray Computer Assisted Tomography for Laboratory Core Studies," Journal of Petroleum Technology (Dec. 1996) 1120-1122.

[90] Higgins R.V. and Leighton, A.J.: "A Computer Method to Calculate Two-Phase Flow in Any Irregularly Bounded Porous Medium," Journal of Petroleum Technology (June 1962) 14, No. 6, 679-683.

[91] Higgins R.V. and Leighton, A.J.: "Computer Prediction of Water Drive of Oil and Gas Mixtures Through Irregularly Bounded Pororus Media - Three-Phase Flow," Journal of Petroleum Technology (September 1962) 14, No. 9, 1048-1054.

[92] Higgins, R.V., Boley, D.W., and A.J. Leighton: "Aids to Forecasting The Performance of Water Floods," Journal of Petroleum Technology (September 1964) 16, 1076-1082.

[93] Hirasaki, G.J.: "Applications of the Theory of Multicomponent, Multiphase Displacement to Three-Component, Two-Phase Surfactant Flooding," Soc. Eng. Pet. Eng. J. (April 1981) 191-204. 
[94] Hirasaki, G.J.: "Structural Interactions in the Wetting and Spreading of Van Der Waals Fluids," Journal of Adhesion Science and Technology (1993) 7, 285-322.

[95] Holden, L.: "On the Strict Hyperbolicity of the Buckley-Leverett Equations for Three-Phase Flow in a Porous Medium," SIAM J. Applied Math. (1990) 50, 667-682.

[96] Holm, L. and Josendal, V.: "Effect of Oil Composition on Miscible-Type Displacement by Carbon Dioxide," Soc. Pet. Eng. J. (February 1982) 87-98.

[97] Honarpour, M.M., Cromwell, V., Hatton, D. and Satchwell, R.: "Reservoir Rock Descriptions Using Computed Tomography (CT)," paper SPE 14272 in the proceedings of the 1985 SPE Annual Technical Conference and Exhibition, Las Vegas, NV, September.

[98] Hou, T. and Wu, X.: "A multiscale finite element method for elliptic problems in composite materials and porous media," Journal of Computational Physics (1997) 134, No. CP975682, $169-189$.

[99] Hough, D.B. and White, L.R.: "The Calculation of Hamaker Constants from Lifshitz Theory with Applications to Wetting Phenomena," Adv. Colloid 6 Interface Sci. (1980) 14, 3-41.

[100] Hutchinson, C.A. Jr. and Braun, P.H.: "Phase Relations of Miscible Displacement in Oil Recovery," A.I.Ch.E. J. (March 1961) 64-72.

[101] Intera Information Technologies Limited: Eclipse 100 Reference Manual - 95A Release, Intera Information Technologies Limited, Henly-on-Thames, Oxfordshire RG9 4PS, England (1994).

[102] Jensen, F. and Michelsen M.L.: "Calculation of First Contact and Multiple Contact Minimum Miscibility Pressures," IN SITU (January 1990) 14, No. 1, 1-17.

[103] Jerauld, G.R.: "Prudhoe Bay Gas/Oil Relative Permeability," SPE Reservoir Engineering (1997) 12, 66-73.

[104] Jerauld, G.R. and Salter, S.J.: "The Effect of Pore-Structure on Hysteresis in Relative Permeabilty and Capillary Pressure: Pore-Level Modeling," Transport in Porous Media (1990) 5, 103-151.

[105] Jessen, K., Bleriot, L., Michelsen, M.L. and Stenby, E.: "Calculation of MMP," International Conference on Production of Reservoir Fluids in Frontier Conditions, Rueil-Malmaison, France (Dec. 4-5 1997).

[106] Jessen, K., Michelsen, M.L. and Stenby, E.H.: "Global Approach for Calculation of Minimum Miscibility Presure," Fluid Phase Equilibria (1998) 153, 251-263.

[107] Johns, R.T.: Analytical Theory of Multicomponent Gas Drives With Two-Phase Mass Transfer, PhD dissertation, Stanford University, Stanford, CA (1992).

[108] Johns, R.T. and Orr, F.M., Jr.: “Miscible Gas Displacement of Multicomponent Oils," $S P E$ Journal (1996) 1, 39-50.

[109] Johns, R.T., B. Dindoruk, and Orr, F.M. Jr.: "Analytical Theory of Combined Condensing/Vaporizing Gas Drives," SPE Advanced Technology Series (May 1993) 1, No. 2, 7-16.

[110] Johns, R.T., Fayers, F.J. and Orr, F.M., Jr.: "Effect of Gas Enrichment and Dispersion on Nearly Miscible Displacements in Condensing/Vaporizing Drives," SPE Advanced Technology Series (1994) 2, No. 2, 173-180. 
[111] Johnson, E.F., Bossler, D.P., and Naumann, V.O.: "Calculation of Relative Permeability from Displacement Experiments," Trans. AIME (1959) 216, 370.

[112] Kalaydjian, F.J.M.: "Performance and Analysis of Three-Phase Capillary Pressure Curves for Drainage and Imbibition in Porous Media," paper SPE 24878 in the proceedings of the 1992 SPE Annual Technical Conference and Exhibition, Washington, DC, October.

[113] Kalaydjian, F.J.M., Moulu, J.C., Vizika, O., and Munkerud, P.K.: "Three Phase Flow in Water-Wet Porous Media: Determination of Gas/Oil Relative Permeabilities Under Various Spreading Conditions," paper SPE 26671 in the proceedings of the 1993 SPE Annual Technical Conference and Exhibition, Houston, TX, October.

[114] Kantzas, A., Chatzis, I. and Dullien, F.A.L.: "Enhanced Oil Recovery by Inert Gas Injection," paper SPE 17379 in the proceedings of the 1988 Sixth SPE/DOE Symposium on Enhanced Oil Recovery, Tulsa, OK, April.

[115] Keller, A.A., Blunt, M.J. and Roberts, P.V.: "Micromodel Observation of the Role of Oil Layers in Three Phase Flow," Transport in Porous Media (1997) 26, 277-297.

[116] Kincaid, D. R., Respess, J., and Young, D.: ITPACK 2C: A FORTRAN Package for Solving Large Sparse Linear Systems by Adaptative Accelerated Iterative Methods, University of Texas, Austin (1997).

[117] King, P.: “The Use of Renormalization for Calculating Effective Permeability," Transport in Porous Media (1989) 4, 37-58.

[118] Kovscek, A.R., Wong, H. and Radke C.J.: "A Pore-Level Scenario for the Devolpment of Mixed Wettability in Oil Reservoirs," AIChE J. (1993) 39, 1072-1085.

[119] Krechel, A. and Stueben, K.: "Operator Dependent Interpolation in Algebraic Multigrid," Proceedings of the 5th European Multigrid Conference, Stuttgart (to appear in "Lecture Notes in Computational Science and Engineering, Springer") (Oct. 1-4 1996).

[120] Kumar, A. and Jerauld, G.: "Impacts of Scale-up on Fluid Flow from Plug to Gridblock Scale in Reservoir Rock," 10th SPE/DOE Symposium on Improved Oil Recovery, Tulsa (April 1996) No. SPE 35452.

[121] Kuo, S.S.: "Prediction of Miscibility for the Enriched-Gas Drive Process," paper SPE 14152 in the proceedings of the 1985 SPE Annual Technical Conference and Exhibition, Las Vegas, NV, September.

[122] Lake, L.W.: Enhanced Oil Recovery, first edition, Prentice Hall, Englewood Cliffs, NJ (1989).

[123] Lake, L.W., Johnston, J.R., and Stegemeier, G.L.: "Simulation and Performance Prediction of a Large-Scale Surfactant/Polymer Project," Society of Petroleum Engineers Journal (December 1981) 21, No. 12, 731-739.

[124] Landau, L.D., and Lifshitz, E.M.: Fluid Mechanics, Pergamon Press, Oxford (1959).

[125] Larson, R.G.: "The Influence of Phase Behavior on Surfactant Flooding," Soc. Eng. Pet. Eng. J. (December 1979) 411-422.

[126] Larson, R.G., and Hirasaki, G.J.: "Analysis of the Physical Mechanisms in Surfactant Flooding," Soc. Eng. Pet. Eng. J. (February 1978) 42-58. 
[127] Leverett, M.C. and W.B. Lewis: "Steady Flow of Gas-Oil-Water Mixtures Through Unconsolidated Sands," Transactions of AIME (1941) 142, 107-116.

[128] Lohrenz, J., Bray, B.C. and Clark, C.R.: "The Viscosity of Pure Substances in Dense Gaseous and Liquid Phases," Journal of Petroleum Technology (Oct. 1964) 16, 1171-1176.

[129] Luks, K.D., Turek, E.A. and Baker, L.E.: "Calculation of Minimum Miscibility Pressure," Soc. Pet. Eng. Res. Eng. (November 1987) 501-506.

[130] MacAllister, D.J., Miller, K.C., Graham, S.K. and Yang C-T.: “Application of X-Ray CT Scanning to the Determination of Gas Water Relative Permeabilities," paper SPE 18284 in the proceedings of the 1990 SPE Annual Technical Conference and Exhibition, New Orleans, LA, September.

[131] Mani, V. and Mohanty, K.K.: "Pore-Level Network Modeling of Three-Phase Capillary Pressure and Relative Permeability Curves," paper SPE 38883 in the proceedings of the 1997 SPE Annual Technical Conference and Exhibition, San Antonio, TX, October.

[132] Martin, J.C. and Wegner, R.E.: "Numerical Solution of Multiphase, Two-Dimensional Incompressible Flow Using Streamtube Relationships," Society of Petroleum Engineers Journal (October 1979) 19, 313-323.

[133] Martin, J.C., Woo, P.T., and Wegner, R.E.: "Failure of Stream Tube Methods To Predict Waterflood Performance of an Isolated Inverted Five-Spot at Favorable Mobility Ratios," Journal of Petroleum Technology (February 1973) 25, 151-153.

[134] Mathews, J.L., Emanuel, A.S., and Edwards, K.A.: "Fractal Methods Improve Mitsue Miscible Predictions," Journal of Petroleum Technology (November 1989) 41, 1136-1989.

[135] McDougall, S.R. and Sorbie, K.S.: "The Impact of Wettability on Waterflooding: Pore-Scale Simulation," SPE Reservoir Engineering (1995) 10, 208-213.

[136] Mehra, R.K., Heidemann, R.A., and Aziz, K.: "An Accelerated Successive Substitution Algorithm," The Canadian Journal of Chemical Engineering (August 1983) 61, 590-596.

[137] Metcalfe, R.S. and Yarborough, L.: "The Effect of Phase Equilibria on the CO2 Displacement Mechanism," Soc. Pet. Eng. J. (August 1979) 242-252.

[138] Metcalfe, R.S., Fussell, D.D., and Shelton, J.L.: "A Multicell Equilibrium Separation Model for the Study of Multiple Contact Miscibility in Rich Gas Drives," SPE Journal (1973) 13, $147-155$.

[139] Michelsen, M.L.: “The Isothermal Flash Problem. Part 1. Stability," Fluid Phase Equilibria (1982) 9, 21-40.

[140] Michelsen, M.L.: "Speeding up the Two-Phase PT-Flash with Applicatioon for Calculation of Miscible Displacements," Fluid Phase Equilibria (1998) 143, 1-12.

[141] Minssieux L. and Duquerroix J-P.: "WAG Flow Mechanisms in Presence of Residual Oil," paper SPE 28623 in the proceedings of the 1994 SPE Annual Technical Conference and Exhibition, New Orleans, LA, September.

[142] Mohanty, K.K., and Salter, S.J.: "Multiphase Flow in Porous Media Part 3: Oil Mobilization, Transverse Dispersion and Wettability," paper SPE 12127 in the proceedings of the 1983 SPE Annual Technical Conference and Exhibition, San Francisco, CA, October. 
[143] Monroe, W.W., Silva, M.K., Larsen, L.L. and Orr, F.M. Jr.: "Composition Paths in FourComponent Systems: Effect of Dissolved Methane on 1D $\mathrm{CO}_{2}$ Flood Performance," SPE Reservoir Engineering (August 1990) 5, 423-432.

[144] Muskat, M.: Flow of Homogeneous Fluids, International Human Resources Development Corporation, 137 Newbury Street, Boston MA 02116 (1937, 1982).

[145] Muskat, M.: "The Theory of Potentiometric Models," Trans. AIME (1948) 179, 216-221.

[146] Muskat, M. and Wyckoff, R.: "A Theoretical Analysis of Waterflooding Networks," Trans. AIME (1934) 107, 62-77.

[147] Naylor, P., Sargent, N.C., Crosbie, A.J., Tilsed A.P. and Goodyear S.G.: "Gravity Drainage During Gas Injection," proceedings of the 8th European IOR Symposium in Vienna, Austria (May 15-17 1995).

[148] Neau, E., Avaulee, L. and Jaubert, J.N.: "A New Algorithm for Enhanced Oil Recovery Calculations," Fluid Phase Equilibria (1996) 117, 265-272.

[149] Newley, T.M.J. and Begg, S.H.: "Characterizing the Effects of Heterogeneity on Oil Recovery by Dry Gas Injection," paper SPE 24921 in the proceedings of the 1992 ATCE of the SPE, Washington, DC, October 4-7.

[150] Newley, T.M.J. and Merrill R.C. Jr.: "Pseudocomponent Selection for Compositional Simulation," Soc. Pet. Eng. Res. Eng. (November 1991) 6, No. 4, 490-496.

[151] Nilsen, L.S., Øren, P.E., Bakke, S. and Henriquez, A.: "Prediction of Relative Permeability and Capillary Pressure from a Pore Model," paper SPE 35531 in the proceedings of the 1996 European 3-D Reservoir Modelling Conference, Stavanger, Norway, April.

[152] Nordtvedt, J.E., Ebeltoft, E., Iverson, J.E., Sylte, A., Urkedal, H., Vatne, K.O. and Watson, A.T.: "Determination of Three-Phase Relative Permeabilities from Displacement Experiments," paper SPE 36683 in the proceedings of the 1996 SPE Annual Technical Conference and Exhibition, Denver, CO, October.

[153] Nouar, A. and Flock, D.L.: "Prediction of the Minimum Miscibility Predictions of a Vaporizing Gas Drive," paper SPE 15075 in the proceedings of the 1986 56th California Regional Meeting of the SPE, Oakland, CA.

[154] Oak, M.J.: "Three-Phase Relative Permeability of Water-Wet Berea," paper SPE 20183 in the proceedings of the 1990 Seventh SPE/DOE Symposium on Enhanced Oil Recovery, Tulsa, OK, April.

[155] Oak, M.J., Baker, L.E. and Thomas, D.C.: "Three-Phase Relative Permeability of Berea Sandstone," Journal of Petroleum Technology (1990) 42, 1057-1061.

[156] Øren, P. E. and Pinczewski, W. V.: "The Effect of Wettability and Spreading Coefficients on the Recovery of Waterflood Residual Oil by Miscible Gasflooding," paper SPE 24881 in the proceedings of the 1992 SPE Technical Conference and Exhibition, Washington, D.C.

[157] Øren, P.-E., Bakke, S. and Arntzen, O.J.: "Extending Predictive Capabilities to Network Models," SPE Journal (1998) 3, 324-336.

[158] Øren, P. E., Billiotte, J. and Pinczewski, W. V.: "Mobilization of Waterflood Residual Oil by Gas Injection for Water-Wet Conditions," SPE Formation Evaluation (March 1992) 70-78. 
[159] Øren, P.E. and Pinczewski, W.V.: "The Effect of Film-Flow on the Mobilization of Waterflood Residual Oil by Gas Flooding," proceedings of the 1991 6th European IOR Symposium, Stavanger, Norway (May 1991).

[160] Øren, P.E. and Pinczewski, W.V.: "Fluid Distribution and Pore Scale Displacement Mechanisms in Drainage Dominated Three-Phase Flow," Transport in Porous Media (1995) 20, $105-133$.

[161] Øren, P.E., Bakke, S., Nilson, L.S. and Henriquez, A.: "Prediction of Relative Permeability and Capillary Pressure from Pore-Scale Modelling," proceedings of the 1996 5th European Conference on the Mathematics of Oil Recovery, Leoben, Austria (Sept. 1996).

[162] Øren, P.E., Billiote, J. and Pinczewski, W.V.: "Pore-Scale Network Modeling of Waterflood Residual Oil Recovery by Immiscible Gas Flooding," paper SPE 27814 in the proceedings of the 1994 SPE/DOE Improved Oil Recovery Symposium,, Tulsa, OK, April.

[163] Orr, F.M., Jr. and Silva, M.K.: "Effect of Oil Composition on Minimum Miscibility Pressure - Part 2: Correlation," Soc. Pet. Eng. Res. Eng. J. (November 1987) 479-491.

[164] Orr, F.M., Jr., Johns, R.T. and Dindoruk, B.: "Miscibility in Four-Component Vaporizing Gas Drives," SPE Reservoir Engineering (1993) 8, No. 28, 135-142.

[165] P. Renard, G. d. M.: "Calculating Equivalent Permeability: A Review," Advances in Water Resources (1997) 20, 253-278.

[166] Pande, K.K. and Orr, F.M., Jr.: "Effect of Viscous Crossflow on Miscibility Development in a Two-Layer Flow System: Part I - Ternary Condensing Gas Drives," SPE Advanced Technology Series (1994) 2, No. 2, 7-17.

[167] Pande, K.K. and Orr, F.M., Jr.: "Effect of Viscous Crossflow on Miscibility Development in a Two-Layer Flow System: Part II - Ternary Vaporizing Gas Drives," SPE Advanced Technology Series (1994) 2, No. 2, 7-17.

[168] Parker, J. C., Lenhard, R. J., and Kuppusamy, T.: "A Parametric Model for Constitutive Properties Governing Multiphase Flow in Porous Media," Water Resources Research (1987) 23, 618-624.

[169] Parsons, R.W.: "Directional Permeability Effects in Developed and Unconfined Five-Spots," Journal of Petroleum Technology (April 1972) 24, 487-494.

[170] Peaceman, D.: "Interpretation of Well-Block Pressures in Numerical Reservoir Simulation," SPEJ (June 1978) 18, 183-194.

[171] Peaceman, D.: "Effective Transmissibilities of a Gridblock by Upscaling-Comparison of Direct Methods with Renormalization," SPEJ (1997) 2, 338-349.

[172] Peddibhotla, S., Cubillos, H., Datta-Gupta, A., and Wu, C.H.: "Rapid Simulation of Multiphase Flow Through Fine-Scale Geostatistical realizations Using a New, 3D, Streamline Model: A Field Example," paper SPE 36008 in the proceedings of the 1996 Petroleum Computer Conference, Dallas, TX.

[173] Peddibhotla, S., Datta-Gupta, A., and Xue, G.: "Multiphase Streamline Modeling in Three Dimensions: Further Generalizations and a Field Application," paper SPE 38003 in the proceedings of the 1997 14th Reservoir Simulation Symposium, Dallas, TX. 
[174] Pedersen, K.S., Fredenslund, A.A. and Thomassen, P.: Properties of Oils and Natural Gases, Gulf Publishing Company, Houston, TX (1989).

[175] Pederson, K.S., Fjellerup, J., Thomassen, P. and Fredenslund, A.: "Studies of Gas Injection into Oil Reservoirs by a Cell-to-Cell Simulation Model," paper SPE 15599 in the proceedings of the 1986 SPE Annual Technical Conference and Exhibition, New Orelans, LA, October.

[176] Peneloux, A., Rauzy, E. and Freze, R.: "A Consistent Correction for Redlich-Kwong-Soave Volumes," Fluid Phase Equilibria (1982) 8, 7-23.

[177] Peng, D.Y. and Robinson, D.B.: "A New Two-Constant Equation of State," Ind. Eng. Chem. Fund. (1976) 15, 59-64.

[178] Pereira, G.G., Pinczewski, W.V., Chan, D.V.C., Paterson L. and Øren, P.E.: "Pore-Scale Network Model for Drainage Dominated Three-Phase Flow in Porous Media," Transport in Porous Media (1996) 24, 167-201.

[179] Pickup, G. and Sorbie, K.: "The Scaleup of Two-Phase Flow in Porous Media Using Phase Permeability Tensors," SPEJ (1996) 1, 369-381.

[180] Pollock, D.W.: "Semianalytical Computation of Path Lines for Finite-Difference Models," Ground Water (November-December 1988) 26, No. 6, 743-750.

[181] Rachford, H.H., Jr. and Rice, J.D.: "Procedure for use of Electronic Digital Computers in Calculations for Multicomponent Vapor-Liquid and Liquid-Liquid Equilibrium," Journal of Petroleum Technology (1952) 4, No. 10.

[182] Ramé, M. and Killough, J.E.: "A New Approach to Flow Simulation in Highly Heterogeneous Porous Media," SPE Formation Evaluation (Sept 1992) 7, No. 3, 247-254.

[183] Ransohoff, T.C. and Radke, C.J.: "Laminar Flow of a Wetting Liquid Along the Corners of a Predominantly Gas-Occupied Noncircular Pore," J. Colloid 63 Interface Sci. (1988) 121, $392-401$.

[184] Renard, G.: "A 2D Reservoir Streamtube EOR Model with Periodical Automatic Regeneration of Streamlines," In Situ (1990) 14, No. 2, 175-200.

[185] Rubin, B. and Edwards, M.: "Extension of the TVD Midpoint Scheme to Higher-Order Accuracy in Time," paper SPE 25265 in the proceedings of the 1993 SPE Symposium on Reservoir Simulation, New Orleans, LA, February.

[186] Sahni, A., Burger, J.E. and Blunt, M.J.: "Measurement of Three Phase Relative Permeability during Gravity Drainage using CT Scanning," paper SPE 39655 in the proceedings of the 1998 SPE/DOE Improved Oil Recovery Symposium, Tulsa, OK, April.

[187] Salathiel, R.A.: "Oil Recovery by Surface Film Drainage in Mixed Wettability Rocks," Journal of Petroleum Technology (1973) 25, 1216-1224.

[188] Sammon, P.H.: “An Analysis of Upstream Differencing," SPE Reservoir Engineering (1988) 3, 1053-1056.

[189] Sarem, A.M.: "Three-Phase Relative Permeability Measurements by Unsteady-State Method," SPE Journal (1966) 6, 199-205. 
[190] Sato, K. and Abbaszadeh, M.: "Tracer Flow and Pressure Performance of Reservoirs Containing Distributed Thin Bodies," SPE Formation Evaluation (September 1996) 11, No. 3, $185-193$.

[191] Shyeh-Yung, J. and Stadler, M.: "Effect of Injectant Composition and Pressure on Displacement of Oil by Enriched Hydrocarbon Gases," SPE Reservoir Engineering (May 1995) 10, No. 2, 109-115.

[192] Siddiqui, S., Hicks, P.J., and Grader, A.S.: "Verification of Buckley Leverett Three-Phase Theory Using Computerized Tomography," J. Pet. Sci. Eng. (1996) 15, 1-21.

[193] Skauge, A., Eleri, O. O., Graue, A. and Monstad, P.: "Influence of Connate Water on Oil Recovery by Gravity Drainage," paper SPE 27817 in the proceedings of the 1994 SPE/DOE Symposium on Improved Oil Recovery, Tulsa, OK, Apr. 17-20.

[194] Skurdal, H., Hustad O. St., and Holt, T.: "Oil Recovery by Gravity Drainage During Gas Injection," proceedings of the 8th European IOR Symposium in Vienna, Austria (May 15-17 1995).

[195] Soll, W.E. and Celia, M.A.: "A Modified Percolation Approach to Simulating Three-Fluid Capillary Pressure-Saturation Relationships," Advances in Water Resources (1993) 16, 107126.

[196] Soll, W.E., Celia, M.A. and Wilson, J.L.: "Micromodel Studies of Three-Fluid Porous Media Systems: Pore-Scale Processes Relating to Capillary Pressure-Saturation Relationships," Water Resources Research (1993) 29, No. 9, 2963-2974.

[197] Stalkup, F.I.: "Miscible Displacement," Monograph 8, Soc. Pet. Eng. of AIME, New York (1983).

[198] Stalkup, F.I.: "Displacement Behavior of the Condensing/Vaporizing Gas Drive Process," paper SPE 16715 in the proceedings of the 1987 SPE Annual Technical Conference and Exhibition, Dallas, TX, September.

[199] Stalkup, F.I.: "Effect of Gas Enrichment and Numerical Dispersion on Enriched-Gas-Drive Predictions," SPE Reservoir Engineering (November 1990) 5, No. 4, 647-655.

[200] Stone, H.L.: "Probability Model for Estimating Three-Phase Relative Permeability," Journal of Petroleum Technology, Trans. AIME (1970) 249, 214-218.

[201] Stone, H.L.: "Estimation of Three-Phase Relative Permeability and Residual Data," Journal of Canadian Petroleum Technology (1973) 12, 53-61.

[202] Stueben, K.: "Algebraic Multigrid (AMG): Experiences and Comparisons," Appl. Math. Comp. Proceedings of the International Multigrid Conference (1983) 13, 419-452.

[203] Tchelepi, H. and Orr, F.M., J.: "Interaction of Viscous Fingering, Permeability Heterogeneity, and Gravity Segregation in Three Dimensions," SPE Reservoir Engineering (November 1994) 9, No. 4, 266-271.

[204] Thiele, M.R., Batycky, R.P., and Blunt, M.J.: “A Streamline-Based 3D Field-Scale Compositional Reservoir Simulator," paper SPE 38889 in the proceedings of the 1997 SPE Annual Technical Conference and Exhibition, San Antonio, TX, Oct. 5-8. 
[205] Thiele, M.R., Batycky, R.P., Blunt, M.J., and Orr, F.M.: "Simulating Flow in Heterogeneous Media Using Streamtubes and Streamlines," SPE Reservoir Engineering (February 1996) 10, No. 1, 5-12.

[206] Thiele, M.R., Blunt, M.J., and Orr, F.M.: "Modeling Flow in Heterogeneous Media Using Streamtubes - I. Miscible and Immiscible Displacements," In Situ (August 1995) 19, No. 3, 299-339.

[207] Thiele, M.R., Blunt, M.J., and Orr, F.M.: "Modeling Flow in Heterogeneous Media Using Streamtubes - II. Compositional Displacements," In Situ (November 1995) 19, No. 4, 367391.

[208] Thiele, M.R., Rao, S.E., and Blunt, M.J.: "Quantifying Uncertainty in Reservoir Performance Using Streamtubes," Mathematical Geology (October 1996) 28, No. 7, 843-856.

[209] Todd, M.R. and Longstaff, W.J.: "The Development, Testing and Application of a Numerical Simulator for Predicting Miscible Flood Performance," Trans., AIME (1972) 253, 874-882.

[210] Trangenstein, J.A.: "Three-Phase Flow with Gravity," Contemporary Mathematics (1989) 100, 147-159.

[211] van Genuchten, M.T.: "A Closed-form Equation for Predicting the Hydraulic Conductivity of Unsaturated Soils," Soil Science Society of American Journal (1980) 44, 892-898.

[212] van Leer, B.: "Towards the Ultimate Conservative Difference Scheme, II. Monotonicity and Conservation Combined in a Second Order Scheme," Journal of Computational Physics (1974) 14, 361-370.

[213] Vasco, D.W., Datta-Gupta, and A., Long, J.C.S.: "Integrating Field Production History in Stochastic Reservoir Characterization," paper SPE 36567 in the proceedings of the 1996 SPE Annual Technical Conference and Exhibition, Denver, CO, October.

[214] Verdière, S. and Guérillot, D.: "Dual Mesh Method in Heterogeneous Porous Media," 5th European Conference on the Mathematics of Oil Recovery, Leoben (Sept. 1996).

[215] Verdière, S. and Vignal, M.: "Numerical and Theoretical study of a dual mesh method using finite volume schemes for two phase flow problems in porous media," Numerische Mathematik (1998) 80, 601-639.

[216] Virnovsky, G.A.: "Determination of Relative Permeabilities in Three-Phase Flow in a Porous Medium," Izv. Akad. Nauk SSSR, Mekh. Zhidk. Gaza (1984) 5, 187-189.

[217] Vizika, O. and Lombard, J-M.: "Wettability and Spreading: Two Key Parameters in Oil Recovery With Three-Phase Gravity Drainage," SPE Reservoir Engineering (1996) 11, 5460 .

[218] Wachmann, C: “The Mathematical Theory for the Displacement of Oil and Water by Alcohol," Society of Petroleum Engineers Journal (September 1964) 231, 250-266.

[219] Walsh, B.W. and Orr, F.M. Jr.: "Prediction of Miscible Flood Performance: The Effect Of Dispersion on Composition Paths in Ternary Systems," In Situ (1990) 14, No. 1, 19-47.

[220] Wang, Y. and Orr, F.M., Jr.: "Analytical Calculation of Minimum Miscibility Presure," Fluid Phase Equilibria (1997) 139, 101-124. 
[221] Wang, Y. and Orr, F.M., Jr.: "Calculation of Mininum Miscibility Pressure," paper SPE 39683 in the proceedings of the 1998 SPE/DOE Symposium on Improved Oil Recovery, Tulsa, OK, Apr. 19-22.

[222] Welge, H.J.: "Displacement of Oil from Porous Media by Water and Gas," Trans. AIME (1950) 198, 314-316.

[223] Welge, H.J.: "A Simplified Method for Computing Oil Recovery by Gas or Water Drive," Trans., AIME (1952) 195, 91-98.

[224] Wellington, S.L. and Vinegar, H.J.: "X-Ray Computerized Tomography," Journal of Petroleum Technology (Aug. 1987) 885-898.

[225] Whitson, C.H. and Michelsen, M.L.: “The Negative Flash," Fluid Phase Equilibria (1989) 53, 51-71.

[226] Wilson G.: "A Modified Redlich-Kwong Equation of State. Application to General Physical Data Calculations," Paper 15c, American Inst. of Chem. Eng. National Meeting, Cleveland, $\mathrm{OH}(1968)$.

[227] Zhou, D. and Blunt, M.J.: "Effect of Spreading Coefficient on the Distribution of Light Non-Aqueous Phase Liquid in the Subsurface," Journal of Contaminant Hydrology (1997) 25, 1-19.

[228] Zhou, D. and Blunt, M.J.: "Wettability Effects in Three-Phase Gravity Drainage," Journal of Petroleum Science and Engineering (1998) 20, 203-211.

[229] Zhou, D., Blunt, M.J. and Orr, F.M., Jr.: "Hydrocarbon Drainage Along Corners of Noncircular Capillaries," Journal of Colloid and Interface Science (1997) 187, 11-21.

[230] Zick, A.A.: "A Combined Condensing/Vaporizing Mechanism in the Displacement of Oil by Enriched Gas," paper SPE 15493 in the proceedings of the 1986 SPE Annual Technical Conference and Exhibition, New Orleans, LA, October. 